Prepared for the U.S. Department of Energy

under Contract DE-AC05-76RL01830

\title{
Thermodynamic Data for
} Geochemical Modeling of

Carbonate Reactions Associated

with $\mathrm{CO}_{2}$ Sequestration -

Literature Review

\author{
KM Krupka \\ KJ Cantrell \\ BP McGrail
}

September 2010

Pacific Northwest

NATIONAL LABORATORY

Proudly Operated by Battelle Since 1965 


\title{
DISCLAIMER
}

This report was prepared as an account of work sponsored by an agency of the United States Government. Neither the United States Government nor any agency thereof, nor Battelle Memorial Institute, nor any of their employees, makes any warranty, express or implied, or assumes any legal liability or responsibility for the accuracy, completeness, or usefulness of any information, apparatus, product, or process disclosed, or represents that its use would not infringe privately owned rights. Reference herein to any specific commercial product, process, or service by trade name, trademark, manufacturer, or otherwise does not necessarily constitute or imply its endorsement, recommendation, or favoring by the United States Government or any agency thereof, or Battelle Memorial Institute. The views and opinions of authors expressed herein do not necessarily state or reflect those of the United States Government or any agency thereof.

\author{
PACIFIC NORTHWEST NATIONAL LABORATORY \\ operated by \\ BATTELLE \\ for the \\ UNITED STATES DEPARTMENT OF ENERGY \\ under Contract DE-AC05-76RL01830
}

Printed in the United States of America

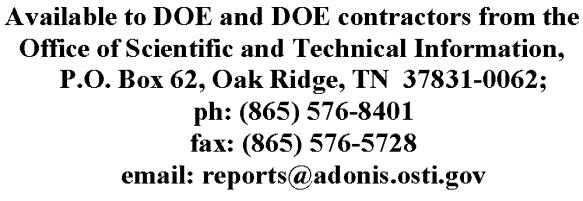

Available to DOE and DOE contractors from the Office of Scientific and Technical Information,

P.O. Box 62, Oak Ridge, TN 37831-0062;

ph: (865) 576-8401

fax: (865) 576-5728

email: reports@adonis.osti.gov

\footnotetext{
Available to the public from the National Technical Information Service, U.S. Department of Commerce, 5285 Port Royal Rd., Springfield, VA 22161 ph: (800) 553-6847 fax: $(703) 605-6900$ email: orders $(a$ ntis.fedworld.gov online ordering: http://www.ntis.gov/ordering.htm
}

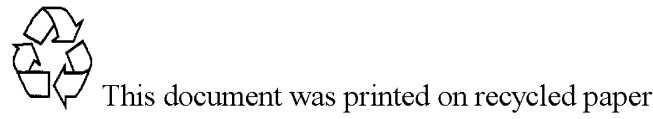




\section{Thermodynamic Data for Geochemical Modeling of Carbonate Reactions Associated with $\mathrm{CO}_{2}$ Sequestration - Literature Review}

KM Krupka

KJ Cantrell

BP McGrail

September 2010

Prepared for

the U.S. Department of Energy

under Contract DE-AC05-76RL01830

Pacific Northwest National Laboratory

Richland, Washington 99352 


\section{Summary}

Permanent storage of anthropogenic $\mathrm{CO}_{2}$ in deep geologic formations is being considered as a means to reduce the concentration of atmospheric $\mathrm{CO}_{2}$ and thus its contribution to global climate change. To assure safe and effective geologic sequestration, numerous studies have been completed regarding the extent to which $\mathrm{CO}_{2}$ migrates within geologic formations, and what physical and geochemical changes occur in these formations when $\mathrm{CO}_{2}$ is injected. Sophisticated, computerized reservoir simulations are used as part of field site and laboratory $\mathrm{CO}_{2}$ sequestration studies. These simulations use coupled multiphase flow-reactive chemical transport models and/or standalone (i.e., no coupled fluid transport) geochemical models to calculate gas solubility, aqueous complexation, reduction/oxidation (redox), and/or mineral solubility reactions related to $\mathrm{CO}_{2}$ injection and sequestration.

Thermodynamic data are critical inputs to modeling geochemical processes. The adequacy of thermodynamic data for carbonate compounds has been identified as an important data requirement for the successful application of these geochemical reaction models to $\mathrm{CO}_{2}$ sequestration. Therefore, a review was completed of thermodynamic data for $\mathrm{CO}_{2}$ gas and carbonate aqueous species and minerals present in published data compilations and databases used in geochemical reaction models. Published studies that describe mineralogical analyses from $\mathrm{CO}_{2}$ sequestration field and natural analogue sites and laboratory studies were also reviewed to identify specific carbonate minerals that are important to $\mathrm{CO}_{2}$ sequestration reactions and therefore require thermodynamic data.

The results of the literature review (Section 5.0) indicate that an extensive thermodynamic database exists for $\mathrm{CO}_{2}$ and $\mathrm{CH}_{4}$ gases, carbonate aqueous species, and carbonate minerals. Values of $\Delta_{\mathrm{f}} \mathrm{G}_{298}{ }^{\circ}$ and/or $\log \mathrm{K}_{\mathrm{r}, 298^{\circ}}$ are available for essentially all of these compounds. However, $\log \mathrm{K}_{\mathrm{r}, \mathrm{T}}{ }^{\circ}$ or heat capacity values at temperatures above $298 \mathrm{~K}$ exist for less than approximately one-third of these compounds. Because the temperatures of host formations that will be used for $\mathrm{CO}_{2}$ injection and sequestration will be at temperatures in the range of $50^{\circ} \mathrm{C}$ to $100^{\circ} \mathrm{C}$ or greater, the lack of high-temperature thermodynamic values for key carbonate compounds, especially minerals, will impact the accuracy of some modeling calculations.

In comparison to the available thermodynamic data for carbonate minerals, only a small number of carbonate minerals have been described in published studies as actually being present in the host rock formations at $\mathrm{CO}_{2}$ injection test sites or natural analogue sites, reaction products of $\mathrm{CO}_{2}$ injection/ intrusion into a host formation, or as mineral reactants or products of laboratory $\mathrm{CO}_{2}$ fluid-rock and -mineral experiments. Except for a few carbonate solid-solution minerals (ferroan and magnesian carbonates and ferroan dolomite), thermodynamic data are available for the pure carbonate minerals identified in these natural analogue site and fluid-rock and -mineral laboratory studies. This suggests that the thermodynamic data for the pure common carbonate minerals is at least adequate for geochemical reaction modeling of $\mathrm{CO}_{2}$ applications. However, more thorough mineralogical characterization in the future might show greater diversity of carbonate mineralogy in host formations used for $\mathrm{CO}_{2}$ sequestration.

Carbonate mineral solid solutions are common and have been extensively studied. It was not possible from our literature review to assess the importance of solid solutions relative to adequately modeling $\mathrm{CO}_{2}$ 
sequestration processes and mineral trapping reactions. Given how common carbonate solid solutions are in nature, one would have to assume inclusion of carbonate solid solutions would be paramount to accurately modeling $\mathrm{CO}_{2}$ sequestration systems.

Two general approaches are used in geochemical reaction models to calculate activity coefficients of aqueous species. The first type consists of the Debye-Hückel equation and its variant, the Davies equation and its extended B-dot equation form. However, these equations are typically limited in accuracy to modeling relatively dilute solutions with ionic strengths less than that of seawater. The second approach involves the use of virial equations, which are sometimes called specific interaction equations or Pitzer equations. Pitzer equations can be applied accurately to systems to high-ionic strengths such as brines and highly saline groundwaters. Application of geochemical models that include the Pitzer equations is important to accurately model in $\mathrm{CO}_{2}$ sequestration in saline formations. However, because redox reactions, especially for the $\mathrm{Fe}(\mathrm{II}) / \mathrm{Fe}(\mathrm{III})$ system, and $\mathrm{Al}$ system have not been fully parameterized for the Pitzer model, use of the Pitzer method currently will not adequately address Fe- or Al-containing geochemical reactions associated with certain $\mathrm{CO}_{2}$ sequestration geochemical systems. 


\section{Acknowledgments}

This research was supported by the Zero Emissions Research \& Technology Center, which is directed by Montana State University and funded by the U.S. Department of Energy, Office of Fossil Energy.

Pacific Northwest National Laboratory is operated by Battelle Memorial Institute for the U.S. Department of Energy under contract DE-AC05-76RL01830. 


\section{Acronyms and Abbreviations}

$\begin{array}{ll}\begin{array}{l}\text { amorphous solid } \\ \text { aq }\end{array} & \begin{array}{l}\text { amorphous solid } \\ \text { aqueous }\end{array} \\ \mathrm{cr} & \text { crystalline solid compound } \\ \mathrm{g} & \text { gas } \\ \mathrm{LLNL} & \text { Lawrence Livermore National Laboratory } \\ \text { PNL } & \text { Pacific Northwest Laboratory } \\ \text { PNNL } & \text { Pacific Northwest National Laboratory } \\ \text { redox } & \text { reduction/oxidation } \\ \text { REE } & \text { rare earth element } \\ \text { S } & \text { solid compound } \\ \text { scCO } & \text { supercritical CO } \\ \text { ss } & \text { solid solution }\end{array}$




\section{Units of Measure}

$\AA$

${ }^{\circ} \mathrm{C}$

cal

$\mathrm{C}_{\mathrm{p}}{ }^{\circ}$

${ }^{\circ} \mathrm{F}$

$\Delta_{\mathrm{f}} \mathrm{G}_{298^{\circ}}$

$\Delta_{\mathrm{f}} \mathrm{G}_{\mathrm{T}}{ }^{\circ}$

$\Delta_{\mathrm{f}} \mathrm{H}_{298^{\circ}}$

$\Delta_{\mathrm{f}} \mathrm{H}_{\mathrm{T}}{ }^{\circ}$

J

K

$\ln$

$\log$

$\log \mathrm{K}_{\mathrm{r}, 298^{\circ}}$

$\log \mathrm{K}_{\mathrm{r}, \mathrm{T}}{ }^{\circ}$

R

$\mathrm{S}_{298}^{\circ}$

$\mathrm{S}_{\mathrm{T}}{ }^{\circ}$

$\mathrm{V}_{298^{\circ}}$ angstrom, a unit of length equal to $10^{-10}$ meter or $10^{-4}$ micrometer

temperature in degrees Celsius $\left.\left[\mathrm{T}\left({ }^{\circ} \mathrm{C}\right)=\mathrm{T}(\mathrm{K})-273.15\right)\right]$

thermochemical calorie; $1 \mathrm{cal}=4.1840 \mathrm{~J}$

heat capacity at constant pressure in $\mathrm{J} /(\mathrm{mol} \cdot \mathrm{K})$

temperature in degrees Fahrenheit $\left\{\mathrm{T}\left({ }^{\circ} \mathrm{F}\right)=\left[\mathrm{T}\left({ }^{\circ} \mathrm{C}\right) \mathrm{x} 9 / 5\right]+32\right.$

standard molar Gibbs free energy of formation at $298.15 \mathrm{~K}$ and $1 \mathrm{bar}$

standard molar Gibbs free energy of formation at temperature $\mathrm{T}$ in degrees Kelvin and 1 bar

standard molar enthalpy of formation at $298.15 \mathrm{~K}$ and $1 \mathrm{bar}$

standard molar enthalpy of formation at temperature $\mathrm{T}$ in degrees Kelvin and $1 \mathrm{bar}$

joule; $1 \mathrm{~J}=0.239006 \mathrm{cal}$

temperature in degrees (listed without degree symbol) Kelvin $\left[\mathrm{T}(\mathrm{K})=\mathrm{T}\left({ }^{\circ} \mathrm{C}\right)+\right.$ 273.15]

natural logarithm, base $\mathrm{e}=2.71828 \ldots$

common logarithm, base 10

common logarithm of the equilibrium constant of reaction at $298.15 \mathrm{~K}$ and $1 \mathrm{bar}$ common logarithm of the equilibrium constant of reaction at temperature $\mathrm{T}$ in degrees Kelvin and 1 bar

gas constant, $8.31451 \mathrm{~J} /(\mathrm{mol} \cdot \mathrm{K})$

standard molar entropy at $298.15 \mathrm{~K}$ and 1 bar

standard molar entropy at temperature $\mathrm{T}$ in degrees Kelvin and $1 \mathrm{bar}$

volume of $1 \mathrm{~mol}$ of a substance at $298.15 \mathrm{~K}$ and $1 \mathrm{bar}$ in $\mathrm{cm}^{3}$ 


\section{Contents}

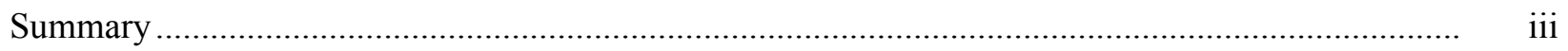

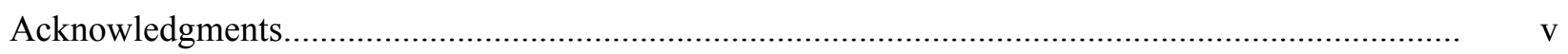

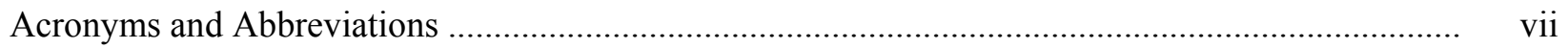

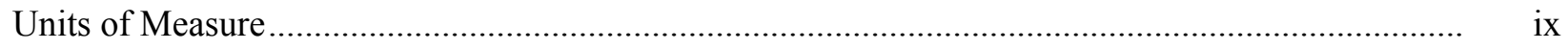

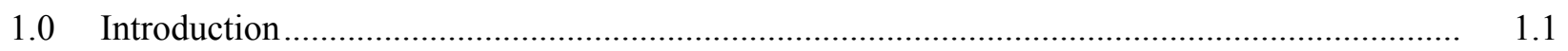

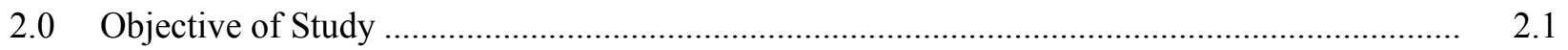

3.0 Thermodynamic Properties Needed for Geochemical Reaction Modeling............................. 3.1

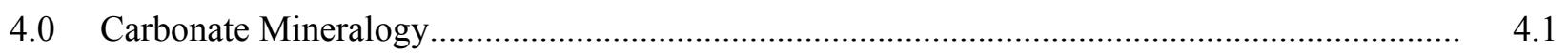

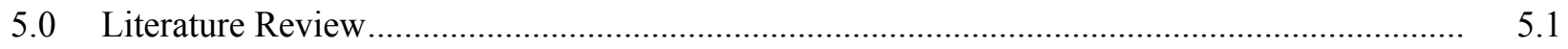

6.0 Geochemical Reaction Models and Thermodynamic Databases Used to Study

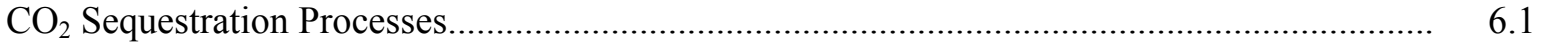

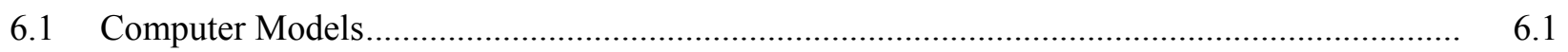

6.2 Principal Sources Containing Thermodynamic Data for Carbonate Species ............................ 6.4

6.3 Availability of Thermodynamic Data for $\mathrm{CO}_{2}$ and $\mathrm{CH}_{4}$ Gases and CarbonateContaining Aqueous Species and Minerals in Identified Databases and

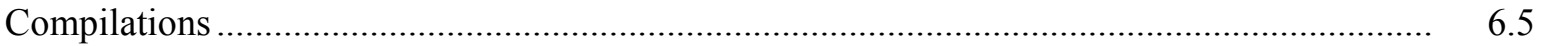

7.0 Comparison of Carbonate Characterization Data to Known Thermodynamic Data.................. 7.1

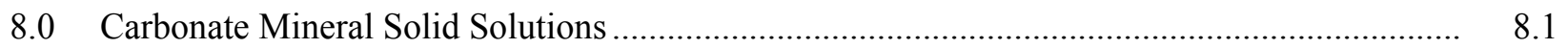

9.0 Importance of Pitzer Approach to Modeling $\mathrm{CO}_{2}$ Sequestration Applications......................... 9.1

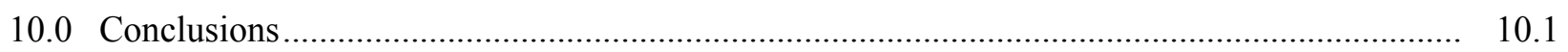

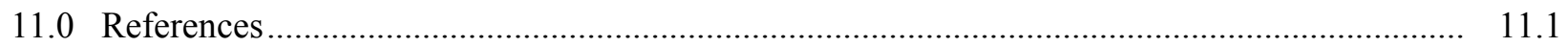

Appendix - Carbonate Minerals ............................................................................................ A.1 


\section{Tables}

4.1 Anhydrous Normal Carbonate Minerals Listed as a Function of Cation Radius

Versus Carbonate Structure Symmetry....

5.1 Computer Modeling Simulation Studies of the Carbonate Reactions Associated with $\mathrm{CO}_{2}$ Sequestration....

5.2 Studies of Mineral Characterization Data and/or Geochemical Analyses at $\mathrm{CO}_{2}$ Field Injection Sites

5.3 Studies of Mineral Characterization Data and/or Geochemical Analyses from Sites Considered Possible Natural Analogues for Carbonate Processes Associated $\mathrm{CO}_{2}$ Injection and Sequestration

5.4 Published Studies of Laboratory Experiments Designed to Examine the Geochemical Reactions Associated with the Interaction of $\mathrm{CO}_{2}$-Containing Fluids with Characterized Rock, Single Mineral, or Cement Samples

6.1 Names of Geochemical Reaction Models Used in the Modeling, Site, and Laboratory $\mathrm{CO}_{2}$ Sequestration Studies Listed in Tables 5.1 through 5.4

6.2 Thermodynamic Data Available for $\mathrm{CO}_{2}$ and $\mathrm{CH}_{4}$ Gases

6.3 Thermodynamic Data Available for Aqueous Carbonate Species.....

6.4 Thermodynamic Data Available for Carbonate-Containing Minerals

7.1 Carbonate Minerals Physically Identified in Host Rock Formations at $\mathrm{CO}_{2}$ Injection or Natural Analogue Sites, Reaction Products of $\mathrm{CO}_{2}$ Injection/Intrusion into a Host Formation, or as Mineral Reactants or Products of Laboratory $\mathrm{CO}_{2}$ Fluid-Rock/ -Mineral Experiments 


\subsection{Introduction}

Permanent sequestration ${ }^{1}$ and storage of $\mathrm{CO}_{2}$ in deep geologic formations is being considered as a means to reduce the concentration of atmospheric $\mathrm{CO}_{2}$ and thus its contribution to global climate change. Types of geologic formations that have been studied for $\mathrm{CO}_{2}$ sequestration include depleted oil and gas fields, deep saline reservoirs, uneconomic coal beds, and basalt formations. The study of $\mathrm{CO}_{2}$ injection and sequestration in depleted oil and gas reservoirs and deep saline formations has received particular attention because of their widespread extent and large storage capacity, typically good permeability for large-scale $\mathrm{CO}_{2}$ injection, and extensive industry experience with enhanced oil and gas recovery in these formations (Bachu and Adams 2003). This review focused on issues related to the computer modeling of geochemical reactions associated with $\mathrm{CO}_{2}$ sequestration in deep saline and basalt formations. Benson and Cole (2008) and Oeklers et al. (2008) present overviews of the $\mathrm{CO}_{2}$ sequestration in sedimentary formations and by basalt carbonation, respectively.

To assure safe and effective geologic sequestration, there have been numerous studies of the extent to which supercritical $\mathrm{CO}_{2}\left(\mathrm{scCO}_{2}\right)$ migrates within geologic formations and what physical and geochemical changes occur in these formations when $\mathrm{CO}_{2}$ is injected. Rochelle et al. (2004) review the geochemical reactions that may occur during $\mathrm{CO}_{2}$ injection in geologic formations and the effect of these reactions on $\mathrm{CO}_{2}$ sequestration. Sequestration of $\mathrm{CO}_{2}$ within geologic media can occur by one or more of the following main processes:

- structural trapping

- hydrodynamic trapping

- solubility and ionic trapping

- mineral trapping.

When $\mathrm{CO}_{2}$ is initially injected into the permeable host (or reservoir) geologic formation, a "bubble" of $\mathrm{CO}_{2}$ is formed that is more buoyant than the saline formation water. This $\mathrm{CO}_{2}$ will then rise through the porous rock until it becomes trapped by an impermeable layer of the caprock or aquitard. This process is known as structural or stratigraphic trapping. Hydrodynamic or residual trapping of $\mathrm{CO}_{2}$ occurs when $\mathrm{CO}_{2}$ becomes trapped as a gas or supercritical fluid in the pore spaces of the reservoir formation and does not move. Because $\mathrm{CO}_{2}$ gas is soluble in dilute and saline waters, injected $\mathrm{CO}_{2}$ will also be trapped when it dissolves in the formation water to form carbonic acid (solubility trapping) and other dissolved carbonate species (ionic trapping). The formation water containing the dissolved $\mathrm{CO}_{2}$ will also react with minerals in the reservoir formation, resulting in dissolution of these primary phases and potentially precipitating carbonate mineral phases in the pore spaces. Because many carbonate minerals [e.g., calcite $\left(\mathrm{CaCO}_{3}\right)$, dolomite $\left[\mathrm{CaMg}\left(\mathrm{CO}_{3}\right)_{2}\right]$, siderite $\left.\left(\mathrm{FeCO}_{3}\right)\right]$ remain stable for geologically significant timeframes, mineral trapping is considered as having the greatest potential for long-term, stable geologic $\mathrm{CO}_{2}$ sequestration (Bachu et al. 1994).

\footnotetext{
${ }^{1}$ Bachu and Adams (2003) define geologic $\mathrm{CO}_{2}$ sequestration and storage “....as the removal of $\mathrm{CO}_{2}$ directly from anthropogenic sources (capture) and its disposal in geologic media, either permanently (sequestration) or for significant time periods (storage)." Sequestration in this report refers to both $\mathrm{CO}_{2}$ sequestration and storage unless a distinction must be made from a technical basis.
} 
In addition to these $\mathrm{CO}_{2}$ trapping processes, there are also other geochemical interactions between the $\mathrm{scCO}_{2}$ fluid, the $\mathrm{CO}_{2}$ /formation water mixture, and the minerals in the reservoir and caprock formations that impact safe and effective $\mathrm{CO}_{2}$ sequestration. These include the dissolution of primary minerals by these fluids and subsequent precipitation of new secondary minerals, including carbonates, in the pore spaces in the reservoir formation at the contact between the reservoir formation and caprock, and in any fractures in the caprock that already exist or are newly formed because of these geochemical processes (Bentham and Kirby 2005). Additional geochemical reactions may also occur at the interface between the $\mathrm{scCO}_{2}$ fluid and $\mathrm{CO}_{2}$ /formation water two-phase system, and minerals in the reservoir formation and in the caprock formation. Kaszuba et al. (2003) observed in laboratory studies the desiccation of the saline formation water when water migrates from the aqueous phase and partitions into the $\mathrm{scCO}_{2}$ phase.

Kaszuba et al. (2003) proposed that the geochemical reactions at these interfaces may differ from the reactions that occur in the bulk geologic media and therefore impact our conceptualization and technical evaluation of geological $\mathrm{CO}_{2}$ sequestration at different sites. For example, they noted that brine desiccation could potentially create a localized environment of higher ionic strength at the interface between the $\mathrm{scCO}_{2}$ and pore water in the reservoir formation (Kaszuba et al. 2003).

Superimposed on the abiotic geochemical interactions described above are the potential roles that subsurface microorganisms might have through biomineralization reactions affecting the effectiveness of $\mathrm{CO}_{2}$ injection, stability of primary minerals in the reservoir and caprock, and precipitation of secondary carbonate minerals. Only nine studies of the effects of microbiological processes on geologic $\mathrm{CO}_{2}$ sequestration could be identified in the journal literature. Mitchell et al. (2009) studied biofilms as a means of reducing the permeability of porous geological matrices to reduce $\mathrm{scCO}_{2}$ leakage and thus enhance the geologic sequestration of $\mathrm{CO}_{2}$. Dupraz et al. (2009a, 2009b) report the results of laboratory studies of $\mathrm{CO}_{2}$ biomineralization processes in saline solutions at conditions associated with deep saline aquifers. To interpret their experimental results, Dupraz et al. (2009b) used the geochemical code CHESS, which was adapted to account for the enzymatically catalyzed ureolysis reaction, the kinetics of gas/solution exchanges , and the rate of $\mathrm{CaCO}_{3}$ precipitation. Power et al. $(2007,2009)$ characterized the weathering regime of the hydromagnesite $\left[\mathrm{Mg}_{5}\left(\mathrm{CO}_{3}\right)_{4}(\mathrm{OH})_{2} \cdot 4 \mathrm{H}_{2} \mathrm{O}\right]$ playas and associated ultramafic bedrock of Atlin, British Columbia, as a natural model for $\mathrm{CO}_{2}$ sequestration from the perspective of biogeochemical processes that accelerate mineral carbonation. The results of other studies of the effects of biomineral-fluid reactions on geologic $\mathrm{CO}_{2}$ sequestration were reported in the recent abstracts by Dupraz et al. (2007, 2008), Martinez et al. (2007), and Mitchell et al. (2008). Given the extensive body of information that currently exists in the literature on the effects of microorganisms on mineral dissolution and precipitation under a variety of subsurface environments (see collection of review papers in Banfield and Nealson [1997]), biomineralization reactions will likely be important to certain geochemical processes associated with geological $\mathrm{CO}_{2}$ sequestration and will thus be a focus of future research.

Geochemical interactions between injected $\mathrm{scCO}_{2}$ and $\mathrm{CO}_{2} /$ formation water and cement present in wellbores is another set of geochemical processes that has received recent attention relative to their importance to geological $\mathrm{CO}_{2}$ sequestration. Cement is used for the construction and completion of $\mathrm{CO}_{2}$ injection and monitoring wells and for abandonment (i.e., closure) of existing wells. Abandoned wells may act as preferential pathways for the leakage of $\mathrm{CO}_{2}$ through the degraded cement used to plug the wells or through openings between the cement casing and the host formation. Cements will degrade when contacted by acids, and this degradation may occur more rapidly under some conditions when contacted by $\mathrm{scCO}_{2}$. This leakage is enhanced by $\mathrm{CO}_{2}$ buoyancy because free-phase $\mathrm{CO}_{2}$ is less dense than the waters in the geologic formations. Carey et al. (2007) have analyzed actual core samples of 
cement taken from an oil well that had been exposed to $\mathrm{CO}_{2}$ injection for several decades. During the past 4 years, results have been reported from several laboratory studies of the mineralogical changes that occur when cement is reacted with $\mathrm{CO}_{2}$-rich aqueous fluids and $\mathrm{scCO}_{2}$ (Bachu and Bennion 2009; BarletGouédard et al. 2007, 2009; Brunet et al. 2009; Centeno et al. 2005, 2007, 2009; Jacquemet et al. 2005, 2008; Kunieda et al. 2008; Kutchko et al. 2007, 2008, 2009; Regnault et al. 2009; Rimmelé et al. 2008; Scherer et al. 2005; Wigand et al. 2009). Some of these studies have included testing of the cement durability and degradation from exposure to $\mathrm{CO}_{2}-\mathrm{H}_{2} \mathrm{~S}$ mixtures (Centeno et al. 2005, 2007, 2009; Jacquemet et al. 2005, 2008). Development of computer models specific to simulating and evaluating the reactivity of cement and its constituent mineral phases to $\mathrm{CO}_{2}$-brine fluid mixtures has started (Carey and Lichtner 2007; Corvisier et al. 2009, 2010; Huet et al. 2006). Well construction and abandonment procedures have changed significantly over the last 100 years and cement quality has also varied significantly over this time frame. For example, new $\mathrm{CO}_{2}$-resistant cement has been introduced and is being tested with respect application of $\mathrm{CO}_{2}$ sequestration (Barlet-Gouédard et al. 2007, 2009). The consequences to geological storage of $\mathrm{CO}_{2}$ from leakage from abandoned wells are discussed by Celia and Bachu (2003), Watson and Bachu (2007), and others. The implications of such leakage from the perspective of $\mathrm{CO}_{2}$ sequestration are beginning to be modeled by researchers, such as Nordbotten et al. (2005).

Sophisticated, computerized reservoir simulations of geologic $\mathrm{CO}_{2}$ sequestration are now conducted routinely as part of field site and laboratory $\mathrm{CO}_{2}$ sequestration studies. Schnaar and Digiulio (2009) review the use of computer modeling techniques in project design, site characterization, assessments of leakage, and site monitoring activities associated with geologic sequestration of $\mathrm{CO}_{2}$. These modeling techniques typically use coupled multiphase flow-reactive chemical transport models and/or standalone (i.e., no coupled fluid transport) geochemical models ${ }^{1}$ to calculate gas solubility, aqueous complexation, reduction/oxidation (redox), and/or mineral solubility reactions related to $\mathrm{CO}_{2}$ injection and sequestration. For a review of geochemical reaction models, the reader is referred to Bethke (2008), Loeppert et al. (1995), Carmichael and Eugster (1987), and the references or papers therein.

Of particular note is the recent book by Marini (2007) entitled Geological Sequestration of Carbon Dioxide: Thermodynamics, Kinetics, and Reaction Path Modeling, and the review journal article by Gaus et al. (2008) entitled "Geochemical and Solute Transport Modelling for $\mathrm{CO}_{2}$ Storage, What to Expect From It?" in the International Journal of Greenhouse Gas Control. Marini (2007) reviews in detail the issues associated with the thermodynamic, kinetic, and reaction path modeling of geologic sequestration. Marini includes detailed discussions of the thermodynamic properties of $\mathrm{CO}_{2}$ and the $\mathrm{CO}_{2}-\mathrm{H}_{2} \mathrm{O}$ system; the interactions of $\mathrm{CO}_{2}$ in aqueous solution; the thermodynamic stability of solid product phases; volume changes that accompany carbonation reactions; and the kinetics of dissolution and precipitation reactions of carbonate, silicate, oxide, and hydroxide minerals relevant to $\mathrm{CO}_{2}$ sequestration. Marini (2007) also takes the reader through some modeling exercises to show the reader how to apply reaction path modeling to geological $\mathrm{CO}_{2}$ sequestration, and then describes the results of several $\mathrm{CO}_{2}$ sequestration modeling studies published in the literature.

\footnotetext{
${ }^{1}$ When referring to the capability of calculating these geochemical reactions with a computer model, we will refer to these two groups of models collectively in the remainder of this paper as geochemical reaction models.
} 
Gaus et al. (2008) presents a detailed review with an extensive supporting reference list of the application of coupled multiphase flow-reactive chemical transport and geochemical modeling to $\mathrm{CO}_{2}$ sequestration. They identify the following four application areas (taken essentially verbatim from Gaus et al. [2008]):

- Long-term integrity modeling to assess the ultimate fate of injected $\mathrm{CO}_{2}$ and impact on physical properties

- Injectivity modeling to evaluate if the direct environment of the well is not changing in terms of physical and chemical properties in such a way that the injection rates are affected

- Well integrity modeling to assess the geochemical effects on $\mathrm{CO}_{2}$ on the well completion itself, whether the well is an injection well built for $\mathrm{CO}_{2}$ storage project, or an abandoned well in case of storage in a depleted oil or gas reservoir or in a deep saline aquifer penetrated by exploration or production wells

- Modeling of laboratory or small-scale field experiments for the calibration of geochemical and solute transport models, identification of case specific chemical or physical parameters, and validation testing of newly developed codes.

Gaus et al. (2008) reviews the data requirements and gaps and presents examples of specific progress over the last 10 years with respect to using reactive chemical transport and geochemical modeling in these four application areas. In all four application areas, Gaus et al. (2008) identifies the lack of thermodynamic and kinetic data, especially for precipitating minerals, as a chronic problem in each of these four modeling application areas. Gaus et al. (2008) also identifies several new emerging application areas for reactive chemical transport and geochemical modeling with respect to $\mathrm{CO}_{2}$ sequestration. These include understanding the relationship between fluid flow, geochemistry, and geomechanics; co-injection of other gases with $\mathrm{CO}_{2}$; and geochemical behavior at the pore scale and how dissolution/precipitations reactions impact porosity and permeability (Gaus et al. 2008). 


\subsection{Objective of Study}

Geochemical reaction models require thermodynamic data to calculate the geochemical reactions associated with $\mathrm{CO}_{2}$ sequestration. This is an important data requirement for the successful application of geochemical reaction modeling to $\mathrm{CO}_{2}$ sequestration (Gaus et al. 2008; Marini 2007). The objective of our study was to review the availability of thermodynamic data for $\mathrm{CO}_{2}$ gas and carbonate aqueous species and minerals present in published data compilations and database files used in geochemical reaction models. Our evaluation also discusses issues pertinent to modeling geochemical reactions involving carbonate mineral solid solutions and carbonate solution equilibria in high-ionic strength saline (brine) solutions relevant to $\mathrm{CO}_{2}$ sequestration in deep saline formations.

Thermodynamic data may not exist for some minerals that are important to reactions that are known to occur from field site and laboratory studies of $\mathrm{CO}_{2}$ sequestration. Therefore, a review of published papers that describe mineralogical analyses from field sites and laboratory studies was also completed. This component of our review was needed to identify other important carbon-containing solids that are not included in existing databases but for which thermodynamic values need to be determined and added to databases.

Our evaluation was limited to pure carbonate compounds and carbonate compounds containing common alkali and alkaline-earth elements and the transition elements Fe and $\mathrm{Mn}$. Carbonate compounds containing other transition, rare earth, and radionuclide elements are not included in this assessment because they are not expected to be relevant to the carbonate reactions associated with $\mathrm{CO}_{2}$ sequestration.

These subject areas are obviously are not the only data needs that impact a researcher's ability to accurately model geochemical reactions for $\mathrm{CO}_{2}$ sequestration. We would be remiss in not acknowledging that simulations of geological $\mathrm{CO}_{2}$ sequestration to the relevant time scales are very difficult. Many of the geochemical processes described above, especially those involving dissolution and precipitation of minerals in the reservoir and caprock formations, will take long time periods to reach equilibrium. Therefore, reaction rates for these processes must be included when trying to accurately model these interactions for data from $\mathrm{CO}_{2}$ field injection and natural analogue sites and laboratory studies of $\mathrm{CO}_{2}$ fluid-rock interactions. There is no question that such information is sparse or not well known. Researchers—such as Bateman et al. (2005), Gaus et al. (2008), Marini (2007), Xu et al. (2004), and others-have identified this as an important data need for accurately geochemical modeling of $\mathrm{CO}_{2}$ sequestration. However, we estimate that a review of thermodynamic data availability for carbonate species provides a baseline for other future reviews of related subject areas, such as reaction rates for precipitation and dissolution of carbonate mineral and minerals present in reservoir and caprock formations. 


\subsection{Thermodynamic Properties Needed for Geochemical Reaction Modeling}

Geochemical models require thermodynamic data to calculate the aqueous speciation, redox, mineral and gas solubility reactions associated with laboratory, field-scale, and site-specific studies of geological sequestration. The thermodynamic principles and equations that are the foundation for calculating these geochemical equilibria are discussed in detail by others such as Appelo and Postma (2005), Langmuir (1997), Marini (2007), and Nordstrom and Munoz (1985) and therefore will not be discussed in detail here. However, to provide a basis to discuss what thermodynamic parameters are included in various published compilations of thermodynamic data or computer model thermodynamic databases, a few thermodynamic relationships are presented here. The authors recognize that the following treatment of thermodynamic equations is elementary and that numerous important nuances exist between the acquisition, evaluation, and tabulation of thermodynamic data for gases, liquids, aqueous species, or solids. Some of these nuances will be addressed later in this report. However, a rigorous discussion of all these issues, such as reference and standard states used for thermodynamic data compiled in difference sources, is beyond the scope of this report. For this background information, the reader should review the introductory sections of thermodynamic data compilations such as those by Wagman et al. (1982) and Robie and Hemingway (1972, 1995), as well as references such as Langmuir (1997) and Nordstrom and Munoz (1985).

Fundamental to modeling such geochemical equilibria is the need for thermodynamic values for Gibbs free energy of formation $\left(\Delta_{\mathrm{f}} \mathrm{G}_{\mathrm{T}}{ }^{\circ}\right)$ or for the common logarithm (base 10) of the equilibrium constant of reaction $\left(\log \mathrm{K}_{\mathrm{r}, \mathrm{T}^{\circ}}\right.$ ), for each gaseous, aqueous, liquid, and solid species of interest. Equation (3.1) provides a general reaction (or equilibrium):

$$
\mathrm{aA}+\mathrm{bB}=\mathrm{cC}+\mathrm{dD}
$$

where the lower-case letters refer to the number of moles of reactants $\mathrm{A}$ and $\mathrm{B}$ and products $\mathrm{C}$ and $\mathrm{D}$, the $\log \mathrm{K}_{\mathrm{r}, \mathrm{T}}{ }^{\circ}$ is defined in Equation (3.2):

$$
\log \mathrm{K}_{\mathrm{r}, \mathrm{T}}{ }^{\circ}=-\frac{\Delta_{\mathrm{r}} \mathrm{G}_{\mathrm{T}}{ }^{\mathrm{o}}}{2.303 \mathrm{R} \mathrm{T}}
$$

where $\Delta_{\mathrm{r}} \mathrm{G}_{\mathrm{T}}{ }^{\circ}$ is the change in Gibbs free energy for the specified reaction at temperature $\mathrm{T}, \mathrm{R}$ is the gas constant $[8.31451 \mathrm{~J} /(\mathrm{mol} \cdot \mathrm{K})]$, and $\mathrm{T}$ is temperature in degrees Kelvin. ${ }^{1}$ As most published compilations of thermodynamic data and computer model thermodynamic databases list thermodynamic values at reference conditions of $\mathrm{T}=298.15 \mathrm{~K}\left(25.0^{\circ} \mathrm{C}\right)$ and $\mathrm{P}=1$ bar $\left(10^{5}\right.$ pascals or $\left.0.98 \mathrm{~atm}\right)$, Equation (3.2) becomes Equation (3.3):

$$
\log \mathrm{K}_{\mathrm{r}, 298}{ }^{\circ}=-\frac{\Delta_{\mathrm{r}} \mathrm{G}_{298}{ }^{\circ}}{5709.07}
$$

\footnotetext{
${ }^{1}$ Thermodynamic values are by convention referenced to the Kelvin temperature scale.
} 
where $\Delta_{\mathrm{r}} \mathrm{G}_{298}{ }^{\circ}$ is in $\mathrm{J} / \mathrm{mol}$. For reaction in Equation (3.1), $\Delta_{\mathrm{r}} \mathrm{G}_{298}{ }^{\circ}$ in Equation (3.3) equates to that in Equation (3.4):

$$
\Delta_{\mathrm{r}} \mathrm{G}_{298}{ }^{\mathrm{o}}=\mathrm{c} \Delta_{\mathrm{f}} \mathrm{G}_{298, \mathrm{C}}{ }^{\mathrm{o}}+\mathrm{d} \Delta_{\mathrm{f}} \mathrm{G}_{298, \mathrm{D}}{ }^{\circ}-\mathrm{a} \Delta_{\mathrm{f}} \mathrm{G}_{298, \mathrm{~A}}{ }^{\circ}-\mathrm{b} \Delta_{\mathrm{f}} \mathrm{G}_{298, \mathrm{~B}}{ }^{\circ}
$$

where $\Delta_{\mathrm{f}} \mathrm{G}_{298}{ }^{\circ}$ is the standard molar Gibbs free energy of formation at $298.15 \mathrm{~K}$ for the indicated reactant or product (as shown by capital letter in subscript).

For any substance $i$ at $298.15 \mathrm{~K}$ and $1 \mathrm{bar}, \Delta_{\mathrm{f}} \mathrm{G}_{298}{ }^{\circ}$ is defined in Equation (3.5):

$$
\Delta_{\mathrm{f}} \mathrm{G}_{298}{ }^{\circ}=\Delta_{\mathrm{f}} \mathrm{H}_{298}{ }^{\circ}+\mathrm{T} \Delta_{\mathrm{f}} \mathrm{S}_{298}{ }^{\circ}
$$

where $\Delta_{\mathrm{f}} \mathrm{H}_{298}{ }^{\circ}$ is the standard molar enthalpy of formation and $\Delta_{\mathrm{f}} \mathrm{S}_{298}{ }^{\circ}$ is entropy of formation of substance $i$. The entropy of formation is the difference of $\mathrm{S}_{298}{ }^{\circ}$ for substance $i$ and the sum of the $\mathrm{S}_{298}{ }^{\circ}$ values for its constituent elements in their standard reference states at the specified $\mathrm{T}$ and $\mathrm{P}$ conditions.

Values of $\Delta_{\mathrm{f}} \mathrm{G}_{\mathrm{T}}{ }^{\circ}$ and $\log \mathrm{K}_{\mathrm{r}, \mathrm{T}}{ }^{\circ}$ can be calculated at temperatures other than $298.15 \mathrm{~K}$ if data exist for parameters such as $\Delta_{\mathrm{f}} \mathrm{H}_{\mathrm{T}}{ }^{\circ}$ and $\mathrm{S}_{\mathrm{T}}{ }^{\circ}$ as a function of temperature from $298.15 \mathrm{~K}$ to $\mathrm{T}$ of interest. At temperatures other than $298.15 \mathrm{~K}$, Equation (3.6) is defined as follows:

$$
\Delta_{\mathrm{f}} \mathrm{G}_{\mathrm{T}}{ }^{\mathrm{o}}=\Delta_{\mathrm{f}} \mathrm{H}_{\mathrm{T}}{ }^{\mathrm{o}}+\mathrm{T} \Delta_{\mathrm{f}} \mathrm{S}_{\mathrm{T}}{ }^{\mathrm{o}}
$$

The enthalpy of formation at temperature $\mathrm{T}\left(\Delta_{\mathrm{f}} \mathrm{H}_{\mathrm{T}}{ }^{\circ}\right)$ can be derived from $\Delta_{\mathrm{f}} \mathrm{H}_{298}{ }^{\circ}$ and $\Delta\left(\mathrm{H}_{\mathrm{T}}{ }^{\circ}-\mathrm{H}_{298}{ }^{\circ}\right)$, which is the difference in relative enthalpy for substance $i$ and its constituent elements between temperature $\mathrm{T}$ and 298.15 as shown in Equation (3.7):

$$
\Delta_{\mathrm{f}} \mathrm{H}_{\mathrm{T}}{ }^{\mathrm{o}}=\Delta_{\mathrm{f}} \mathrm{H}_{298}{ }^{\mathrm{o}}+\Delta\left(\mathrm{H}_{\mathrm{T}}{ }^{\mathrm{o}}-\mathrm{H}_{298}{ }^{\mathrm{o}}\right)
$$

The relative enthalpy for substance $i$ at temperature $\mathrm{T}$ in turn is related to its molar heat capacity $\left(\mathrm{C}_{\mathrm{p}}{ }^{\circ}\right)$ measured as a function of temperature from $298.15 \mathrm{~K}$ to $\mathrm{T}$ as shown in Equation (3.8):

$$
\mathrm{H}_{\mathrm{T}}{ }^{\mathrm{o}}-\mathrm{H}_{298}{ }^{\mathrm{o}}=\int_{298}^{\mathrm{T}} \mathrm{C}_{\mathrm{P}}{ }^{\mathrm{o}} \mathrm{dT}
$$

Similarly, the entropy for substance $i$ at temperature $\mathrm{T}$ is related to its molar heat capacity $\left(\mathrm{C}_{\mathrm{p}}{ }^{\circ}\right)$ from 298.15 $\mathrm{K}$ to $\mathrm{T}$ as shown in Equation (3.9):

$$
\mathrm{S}_{\mathrm{T}}{ }^{\mathrm{o}}=\mathrm{S}_{0}{ }^{\mathrm{o}}+\int_{0}^{\mathrm{T}} \frac{\mathrm{C}_{\mathrm{P}}{ }^{\mathrm{o}}}{\mathrm{T}} \mathrm{dT}=\left(\mathrm{S}_{0}{ }^{\mathrm{o}}+\int_{0}^{298} \frac{\mathrm{C}_{\mathrm{P}}{ }^{\mathrm{o}}}{\mathrm{T}} \mathrm{dT}\right)+\int_{298}^{\mathrm{T}} \frac{\mathrm{C}_{\mathrm{P}}{ }^{\mathrm{o}}}{\mathrm{T}} \mathrm{dT}=\mathrm{S}_{298}{ }^{\circ}+\int_{298}^{\mathrm{T}} \frac{\mathrm{C}_{\mathrm{P}}{ }^{\mathrm{o}}}{\mathrm{T}} \mathrm{dT}
$$

where $\mathrm{S}_{0}{ }^{\circ}$ and $\mathrm{S}_{298}{ }^{\circ}$ are the entropies of substance $i$ at absolute zero $(\mathrm{T}=0.0 \mathrm{~K})$ and $298.15 \mathrm{~K}$, respectively.

In the absence of any structural transitions (e.g., change from trigonal to monoclinic) or phase changes (e.g., melting), $\mathrm{C}_{\mathrm{p}}{ }^{\circ}$ is typically a well-behaved function for most substances especially at 
temperatures above $298.15 \mathrm{~K}$. When high temperature $\mathrm{C}_{\mathrm{P}}{ }^{\circ}$ values are reported in the literature, the values are commonly fitted by an empirical equation as a function of temperature (e.g., Robie and Hemingway 1995). These equations can be used to interpolate $C_{P}{ }^{\circ}$ values at any temperature between the minimum and maximum temperatures used for fitting or to easily do the $\mathrm{C}_{\mathrm{P}}{ }^{\circ}$ integrations necessary to derive values for $\Delta\left(\mathrm{H}_{\mathrm{T}}{ }^{\circ}-\mathrm{H}_{298}{ }^{\circ}\right)$ and $\mathrm{S}_{\mathrm{T}}{ }^{\circ}$ as defined by Equations (3.8) and (3.9), respectively. Two functions commonly used to represent $\mathrm{C}_{\mathrm{P}}{ }^{\circ}$ as a function of temperature include what is now referred to as the Maier-Kelly equation (Maier and Kelly 1932):

$$
\mathrm{C}_{\mathrm{P}}^{\mathrm{o}}=\mathrm{a}+\mathrm{bT}+\frac{\mathrm{c}}{\mathrm{T}^{2}}
$$

and an extended form of Equation (3.10)

$$
\mathrm{C}_{\mathrm{P}}^{\mathrm{o}}=\mathrm{a}+\mathrm{bT}+\frac{\mathrm{c}}{\mathrm{T}^{2}}+\frac{\mathrm{d}}{\mathrm{T}^{0.5}}+\mathrm{eT}^{2}
$$

which was suggested by Hass and Fisher (1976). Typically, Equation (3.11) provides a superior fit to highly precise $\mathrm{C}_{\mathrm{P}}{ }^{\circ}$ measurements. However, extrapolations using the extended Equation (3.11) beyond the temperature limits used for fitting are not valid physically, whereas the Maier-Kelly equation can usually be extrapolated with fair predictability for a short temperature range (Krupka et al. 1985). The reader should refer to Nordstrom and Munoz (1985) for a discussion of technical issues related to the fitting of $\mathrm{C}_{\mathrm{P}}{ }^{\circ}$ data sets with empirical equations.

A variety of techniques can be used to measure or estimate the thermodynamic properties of gases, liquids, aqueous species, and solids relevant to geochemical systems. Nordstrom and Munoz (1985) present a good review of these methods. Possible measurement techniques include calorimetric measurements, phase-equilibria studies, volume measurements, solubility measurements, conductivity measurements, and electromotive force measurements. Because spectrophotometric and spectroscopic techniques provide information about the structures of gases, aqueous species, liquids, and solids, data from these methods can also be used to help derive or estimate thermodynamic values. 


\subsection{Carbonate Mineralogy}

This section presents a brief review of mineralogy of the most common carbonate species. It also provides some perspective for later sections that discuss the extent of thermodynamic data available for carbonate minerals. For more detailed reviews of the geochemistry, mineralogy, crystal chemistry, and phase relations of carbonate minerals, readers are referred to such sources as Morse and Mackenzie (1990), Railsback (1999), and the review papers in Reeder (1983b).

Carbonate minerals are one of the most common groups of non-silicate minerals. They are found in several rock types including sedimentary rocks such as limestones, dolomites, evaporites; metamorphosed carbonate sediments such as marbles; and ultrabasic igneous rocks such as carbonatites and serpentinites. Carbonate minerals are also common in soils and sediments. In sandstones and other detrital rocks, carbonate minerals are present as cements between mineral grains, coatings on grains, and/or fillings in the interstitial spaces between grains. The crystal chemistry and phase relations of carbonate sedimentary rocks and secondary carbonate mineralization in detrital rocks are pertinent to understanding the impact of $\mathrm{CO}_{2}$ injection and sequestration to the carbonate geochemistry of the aquifer host rock.

Although most geoscientists know of one or two dozen carbonate minerals, there are at least 277 carbonate-containing minerals (Railsback 1999). Although calcite $\left(\mathrm{CaCO}_{3}\right)$ and dolomite $\left[\mathrm{CaMg}\left(\mathrm{CO}_{3}\right)_{2}\right]$ make up more than $90 \%$ of the natural rock-forming carbonate minerals, Railsback (1999) advises researchers not to limit their thinking and analyses of mineral systems to the familiar divalent cation carbonate minerals. In addition to the natural geological systems that have historically been studied by geoscientists, geoscience research today also includes a diverse range of studies in support of contaminant remediation technologies that are based on mineral systems and of cleanup or siting of surface and subsurface geological systems that have been or will be impacted by anthropogenic activities. Railsback (1999) suggests that more thorough mineralogical characterization by x-ray diffraction, electron microscopy, and various spectroscopy techniques may possibly show greater mineralogical diversity than traditional assumptions would suggest for such environmental systems.

To help show the diversity of carbonate-containing minerals discussed by Railsback (1999), we have included in the appendix listings of mineral names and formula for the following:

- Pure carbonate minerals in which only $\mathrm{HCO}_{3}{ }^{-}, \mathrm{CO}_{3}{ }^{2-}, \mathrm{OH}^{-}$, or $\mathrm{O}^{2-}$ account for all the negative charge

- Carbonate-containing minerals that contain chloride, sulfate, or fluoride

- Carbonate-containing minerals that contain phosphate or silicate

- Carbonate-containing minerals that contain two or more other anions.

These tables were prepared from the extensive listing of carbonate-containing minerals compiled by LB Railsback (Department of Geology, University of Georgia, Athens, Georgia) with help of JA Mandarino (Royal Ontario Museum, Toronto, Ontario, Canada). Railsback's tables are provided online at http://www.gly.uga.edu/railsback/CO3mins_intro.html, and as an appendix to his 1999 paper on "Patterns in the Compositions, Properties, and Geochemistry of Carbonate Minerals" in Carbonates and 
Evaporites (Railsback 1999). Railsback notes that many of the carbonate-containing minerals listed in his tables would not be classified as carbonate minerals but derive at least part of their negative charge from $\mathrm{CO}_{3}{ }^{2-}$ or $\mathrm{HCO}_{3}{ }^{-}$.

To limit the number of minerals listed in the tables in our appendix, the authors of this report did not include any carbonate-containing minerals tabulated by Railsback (1999), which include As, B, Ba, Bi, $\mathrm{Cd}, \mathrm{Ce}, \mathrm{Co}, \mathrm{Cr}, \mathrm{Cu}, \mathrm{F}, \mathrm{Ge}, \mathrm{Hg}, \mathrm{La}, \mathrm{Li}, \mathrm{NH}_{4}, \mathrm{Nb}, \mathrm{Nd}, \mathrm{Ni}, \mathrm{Pb}, \mathrm{REE}, \mathrm{Sr}, \mathrm{Ta} \mathrm{U}, \mathrm{Y}, \mathrm{Zn}$, and/or Zr. This was done because there is an assumed lack of importance of such carbonate-species to $\mathrm{CO}_{2}$ sequestration. However, it should be noted that carbonate-containing minerals containing transition metals and noncarbonate anions may exert important geochemical controls on the solution concentrations and environmental mobility of these metals and non-carbonate anions even though the particular carbonate-containing mineral makes up a small weight percentage of the total mineralogy of the aquifer system.

Carbonate minerals can be grouped as 1) anhydrous normal carbonates, 2) hydrated normal carbonates, 3) acid carbonates (bicarbonates), and 4) compound carbonates containing hydroxide, halide, or other anions in addition to the carbonate. Carbonate minerals are defined as mineral ${ }^{1}$ species that contain the carbonate ion $\left(\mathrm{CO}_{3}{ }^{2-}\right)$ as the fundamental anionic unit. The $\mathrm{CO}_{3}$ group occurs as a rigid structure consisting of one central $\mathrm{C}$ atom surrounded by three $\mathrm{O}$ atoms in a trigonal planar arrangement. Most of the common carbonates belong to the anhydrous normal carbonate group. Carbonate minerals of the other three groups are less common to very rare, and are typically associated with relatively low temperature geological environments.

Calcite $\left(\mathrm{CaCO}_{3}\right)$, dolomite $\left[\mathrm{CaMg}\left(\mathrm{CO}_{3}\right)_{2}\right]$, and aragonite $\left(\mathrm{CaCO}_{3}\right)$ are probably the most commonly known of all the carbonate minerals. Calcite and dolomite are the most abundant and constitute more than $90 \%$ of the natural rock-forming carbonate minerals (Reeder 1983a). Calcite and aragonite are two of five $\mathrm{CaCO}_{3}$ minerals known to naturally exist. There are three crystalline anhydrous $\mathrm{CaCO}_{3}$ polymorphs ${ }^{2}$ - calcite, aragonite, and vaterite, and amorphous anhydrous $\mathrm{CaCO}_{3}$ is also known. The $\mathrm{CaCO}_{3}$ polymorphs and their crystallization and transformation mechanisms are discussed in Carlson (1983), Cölfen (2003), Jiménez-López et al. (2001), Kitamura (2001), Nissenbaum et al. (2008), Ogino et al. (1987, 1990), Perdikouri et al. (2008), and the references therein. Crystals of calcite, aragonite, and vaterite are typically rhombohedral, acicular (needle-like), and polycrystalline spherical, respectively, in morphology. Thermodynamically, calcite is considered the most stable $\mathrm{CaCO}_{3}$ polymorph at ambient conditions. Aragonite is a high pressure, low temperature $\mathrm{CaCO}_{3}$ polymorph, which can also form at ambient pressure and temperature conditions by biomineralization processes. Vaterite is the rarest and most soluble of the three $\mathrm{CaCO}_{3}$ polymorphs. Although vaterite can form under laboratory conditions that produce rapid $\mathrm{CaCO}_{3}$ supersaturation, vaterite is considered metastable with respect to calcite and aragonite and to the geochemical conditions of sedimentary systems (Morse and Mackenzie [1990] and references therein). Morse et al. (2008) have reported the precipitation of vaterite crystals on substrates of Iceland spar calcite in high-ionic strength $\mathrm{NaCl}$ solutions. The vaterite subsequently recrystallized to

\footnotetext{
${ }^{1}$ A mineral is defined as a solid that must have a crystalline structure, must be homogeneous substance with a defined chemical composition, and must have formed as a result of naturally occurring geological processes. Although traditional definitions excluded organically derived solids, modern mineral classifications include an organic class. Naturally occurring solid substances that do not meet this definition are typically referred to as mineraloids. Examples include obsidian, opal, jet, and pearls.

${ }^{2}$ Polymorphism is a characteristic of a chemical substance with a defined composition to crystallize in more than one crystal form.
} 
euhedral calcite crystals. Anhydrous amorphous $\mathrm{CaCO}_{3}$ is a transient phase, which also occurs as a product of biomineralization. Two hydrous $\mathrm{CaCO}_{3}$ minerals also exist; these include ikaite $\left(\mathrm{CaCO}_{3} \cdot 6 \mathrm{H}_{2} \mathrm{O}\right)$ and monohydrocalcite $\left(\mathrm{CaCO}_{3} \cdot \mathrm{H}_{2} \mathrm{O}\right)$. Their formation is favored by low temperatures and high hydrostatic pressures (Morse and Mackenzie 1990).

Besides calcite, dolomite, and aragonite, other commonly known, but less abundant, carbonates include the alkaline earth- and transition metal-containing minerals such as the following:

- ankerite $\left[\mathrm{CaFe}\left(\mathrm{CO}_{3}\right)_{2}\right]$

- artinite $\left[\mathrm{Mg}_{2} \mathrm{CO}_{3}(\mathrm{OH})_{2} \cdot 3 \mathrm{H}_{2} \mathrm{O}\right]$

- azurite $\left[\mathrm{Cu}_{3}\left(\mathrm{CO}_{3}\right)_{2}(\mathrm{OH})_{2}\right]$

- cerussite $\left(\mathrm{PbCO}_{3}\right)$

- huntite $\left[\mathrm{Mg}_{3} \mathrm{Ca}\left(\mathrm{CO}_{3}\right)_{4}\right]$

- hydromagnesite $\left[\mathrm{Mg}_{5}\left(\mathrm{CO}_{3}\right)_{4}(\mathrm{OH})_{2} \cdot 4 \mathrm{H}_{2} \mathrm{O}\right]$

- magnesite $\left(\mathrm{MgCO}_{3}\right)$

- malachite $\left[\mathrm{Cu}_{2} \mathrm{CO}_{3}(\mathrm{OH})_{2}\right]$

- nahcolite $\left(\mathrm{NaHCO}_{3}\right)$

- natrite $\left(\mathrm{Na}_{2} \mathrm{CO}_{3}\right)$

- natron $\left(\mathrm{Na}_{2} \mathrm{CO}_{3} \cdot 10 \mathrm{H}_{2} \mathrm{O}\right)$
- nesquehonite $\left(\mathrm{MgCO}_{3} \cdot 3 \mathrm{H}_{2} \mathrm{O}\right)$

- otavite $\left(\mathrm{CdCO}_{3}\right)$

- rhodochrosite $\left(\mathrm{MnCO}_{3}\right)$

- $\quad$ siderite $\left(\mathrm{FeCO}_{3}\right)$

- $\quad$ smithsonite $\left(\mathrm{ZnCO}_{3}\right)$

- $\quad$ soda (natron) $\left(\mathrm{Na}_{2} \mathrm{CO}_{3} \cdot 10 \mathrm{H}_{2} \mathrm{O}\right)$

- strontianite $\left(\mathrm{SrCO}_{3}\right)$

- thermonatrite $\left(\mathrm{Na}_{2} \mathrm{CO}_{3} \cdot \mathrm{H}_{2} \mathrm{O}\right)$

- trona $\left(\mathrm{Na}_{3} \mathrm{HCO}_{3} \mathrm{CO}_{3} \cdot 2 \mathrm{H}_{2} \mathrm{O}\right)$

- witherite $\left(\mathrm{BaCO}_{3}\right)$

The anhydrous normal carbonates can be divided into two groups depending on the ionic radii of the cation. In one of these two groups, the carbonates crystallize with rhombohedral (trigonal) symmetry (calcite type) in which the cations are in six-fold coordination with the $\mathrm{O}$ atoms. Reeder (1983a) and Goldsmith (1983) discuss in detail with extensive supporting references the crystal chemistry and phase relations, respectively, of rhombohedral carbonates. Calcite and dolomite are examples of carbonates that form in this group. In the second group, the carbonates have orthorhombic symmetry (aragonite type) where the cations are in nine-fold coordination with O atoms. Speer (1983) reviews in detail with extensive supporting references the crystal chemistry and phase relations of orthorhombic carbonates. The six-fold and nine-fold coordination sites in these two carbonate structure groups can accommodate relatively small (e.g., $\mathrm{Fe}^{2+}, \mathrm{Mg}^{2+}, \mathrm{Mn}^{2+}$, and $\mathrm{Zn}^{2+}$ ) and large cations (e.g., $\mathrm{Ba}^{2+}, \mathrm{Pb}^{2+}$, and $\mathrm{Sr}^{2+}$ ), respectively, with $\mathrm{Ca}^{2+}$ fortuitously on the limit of cation sizes for the two groups. This relationship between carbonate structure symmetry and cation sizes is shown by the values listed in Table 4.1.

In the calcite structure group, the divalent atoms are located at the points of a face-centered rhombohedron, and the $\mathrm{CO}_{3}{ }^{2-}$ groups are positioned midway between the $\mathrm{Ca}$ atoms along rows parallel to the rhombohedral cleavage edges. Magnesite, rhodochrosite, siderite, and smithsonite are isostructural ${ }^{1}$ with calcite. In the dolomite structure, there is a regular ordered substitution of alternate Ca atoms along the $c$ axis in the calcite rhombohedral structure by another divalent metal such as $\mathrm{Fe}^{2+}, \mathrm{Mg}^{2+}$, or $\mathrm{Mn}^{2+}$. Examples of carbonates with this structure type include dolomite and ankerite. The highly ordered structure of dolomite is believed to inhibit its precipitation from aqueous solutions at low temperatures.

\footnotetext{
${ }^{1}$ Isostructural is a characteristic of two or more chemical substances with similar crystal structures but do not exhibit polymorphism (i.e., not having the same composition).
} 
Dolomite formation continues to be debated and discussed in further detail in most journal articles and textbooks that address the geochemistry of sedimentary carbonates.

Table 4.1. Anhydrous Normal Carbonate Minerals Listed as a Function of Cation Radius (in $\AA$ ) Versus Carbonate Structure Symmetry

\begin{tabular}{|c|c|c|c|}
\hline Mineral Name & Formula & $\begin{array}{c}\text { Cation Radius }^{(\mathbf{a})} \\
(\AA)\end{array}$ & Structure Symmetry \\
\hline Magnesite & $\mathrm{MgCO}_{3}$ & 0.72 & Rhombohedral \\
\hline Smithsonite & $\mathrm{ZnCO}_{3}$ & 0.74 & Rhombohedral \\
\hline Siderite & $\mathrm{FeCO}_{3}$ & 0.78 & Rhombohedral \\
\hline Rhodochrosite & $\mathrm{MnCO}_{3}$ & 0.83 & Rhombohedral \\
\hline Otavite & $\mathrm{CdCO}_{3}$ & 0.95 & Rhombohedral \\
\hline Calcite & $\mathrm{CaCO}_{3}$ & 1.00 & Rhombohedral \\
\hline Aragonite & $\mathrm{CaCO}_{3}$ & 1.00 & Orthorhombic \\
\hline Strontianite & $\mathrm{SrCO}_{3}$ & 1.18 & Orthorhombic \\
\hline Cerussite & $\mathrm{PbCO}_{3}$ & 1.19 & Orthorhombic \\
\hline Witherite & $\mathrm{BaCO}_{3}$ & 1.35 & Orthorhombic \\
\hline
\end{tabular}

In the aragonite structure, the $\mathrm{Ca}$ atoms lie approximately in the positions of hexagonal close packing that gives aragonite a pseudo-hexagonal character. The $\mathrm{CO}_{3}$ groups form planar trigonal groups parallel to [001], and each $\mathrm{CO}_{3}$ lies between six $\mathrm{Ca}$ atoms. Cerussite, strontianite, and witherite are isostructural with aragonite.

In contrast to Ca-containing carbonates that are mostly anhydrous, there are several hydrous $\mathrm{Mg}$ carbonates. Hydrated carbonates have different crystal structures than anhydrous carbonates and many have monoclinic crystal symmetries. In modern sediments, some hydrous $\mathrm{Mg}$ carbonates are more common that anhydrous magnesite and dolomite. This is estimated to be due to the high energy of hydration of the $\mathrm{Mg}$ ion relative to the Ca ion (Morse and Mackenzie 1990).

Solid solutions of different carbonate minerals are common. A solid solution is defined as a compositional variation of a crystalline substance due to substitution or omission of various atomic constituents within a crystal structure, within limits determined by the degree of deformation allowed by the crystal structure. The extent of solid solution depends upon the charges and relative sizes of the constituent ions, the coexistence and composition of other minerals or liquid, and the temperature and pressure conditions of formation. For the purpose of this report, the term "coprecipitation" refers to the process of the substitutional or interstitial incorporation of trace concentrations of metals (or anions) during the formation of minerals. However, the term "solid solution" is typically used to refer to more extensive compositional variations, such as a few 10 s to a $100 \mathrm{~mol} \%$. When the concentration of a coprecipitate reaches considerable level (e.g., greater than 1\%), its presence in the structure can significantly affect the chemical properties, such as solubility, of that mineral. Morse and Mackenszie (1990) present the general concepts of coprecipitation reactions and solid solutions of carbonate minerals. 
Examples of carbonate solid solutions include the random substitution of $\mathrm{Mg}$ for $\mathrm{Ca}$ in the calcite structure, and $\mathrm{Sr}$ for $\mathrm{Ca}$ in aragonite structure. Calcite containing a range of $\mathrm{Mg}$ in solid solution is usually termed Mg (or magnesian) calcites. Mackenzie et al. (1983) presents a detailed review of Mg calcites. In some instances, biogenic calcites may contain up to $30 \mathrm{~mol} \% \mathrm{Mg}$. Magnesian calcites are usually described as either low Mg-calcites with less than $5 \mathrm{~mol} \% \mathrm{Mg}$, or high $\mathrm{Mg}$-calcites with 5-30 mol\% Mg (Appelo and Postma 2005).

Dolomite also exhibits solid solution. The Ca-to-Mg ratio may vary and substitution of $\mathrm{Fe}$ and $\mathrm{Mn}$ is common in dolomites. However, note that dolomite is structurally different than $50 \mathrm{~mol} \% \mathrm{high} \mathrm{Mg}$ calcite. In dolomite, the $\mathrm{Mg}$ atoms are substituted in a regular order with alternate $\mathrm{Ca}$ atoms along the $c$ axis, whereas the $\mathrm{Ca}$ and $\mathrm{Mg}$ atoms in a $\mathrm{Mg}$ calcite are distributed more or less randomly. Therefore, dolomite should be treated thermodynamically as a pure phase, while a solid solution model applies for the thermodynamic properties of $\mathrm{Mg}$ calcites (Appelo and Postma 2005). Dolomite and ankerite end members also form part of a solid solution series that extends from $\mathrm{CaMg}\left(\mathrm{CO}_{3}\right)_{2}$ to $70 \mathrm{~mol} \% \mathrm{CaFe}\left(\mathrm{CO}_{3}\right)_{2}$. The boundary between dolomite and ankerite is arbitrarily set at $20 \mathrm{~mol} \% \mathrm{CaFe}\left(\mathrm{CO}_{3}\right)_{2}$.

Carbonate mineral solid solutions and their effect on $\mathrm{CO}_{2}$ sequestration reactions are discussed further in a later section of this report. 


\subsection{Literature Review}

As a starting point for this assessment, published literature was reviewed to identify studies pertaining to computer modeling simulations, field injection sites, natural analogue sites, and laboratory fluid-rock and -mineral studies of the carbonate reactions associated with the subsurface injection and sequestration of $\mathrm{CO}_{2}$ and acid gases (co-injection of $\mathrm{H}_{2} \mathrm{~S}$ or $\mathrm{SO}_{2}$ with $\mathrm{CO}_{2}$ ). Our review was extensive and provides a reasonable cross section of the types of geochemical reaction modeling, site, and laboratory studies of $\mathrm{CO}_{2}$ sequestration that have been completed or are ongoing by the research and development community. The literature material included our review is based on literature published primarily through the end of September 2009.

This review was completed by a combination of using online computerized search tools and literature databases and then identifying additional relevant publications by examining the reference lists in publications found through the online searches. However, our review was not intended to be completely comprehensive and capture every published study related to the geochemistry of $\mathrm{CO}_{2}$ sequestration. As typically is the case for such reviews, we likely missed some relevant published studies, such as those published in proceedings from technical meetings or as company technical reports. These types of publications are not indexed for online search tools, referenced in journal publications, and/or not readily available to the public.

Those studies identified via the literature review that pertain to computer modeling simulation, field injection site, natural analogues, and laboratory fluid-rock and -mineral studies of the carbonate reactions are listed in Tables 5.1, 5.2, 5.3, and 5.4, respectively. The studies in Table 5.1 describe the results of essentially modeling simulation studies only, and typically do not present any new site geochemical characterization data from field injection and natural analogue sites or from laboratory fluid-rock or -mineral studies. Many of the studies listed in Tables 5.2, 5.3, and 5.4 also include the application of geochemical reaction models and are so noted. In these studies, geochemical reaction models were used to interpret characterization data from site or laboratory studies and/or complete sensitivity analyses to evaluate how the geochemistry at a site or in the fluid-rock materials in an autoclave might respond to changes in geochemical parameters such as temperature, $\mathrm{CO}_{2}$ pressure, fluid chemistry, and/or mineralogy. Some of the cited studies in Tables 5.1 through 5.4 are only available at the time this report was published (September 2010) as extended abstracts. These abstracts were published in Geochimica et Cosmochimica Acta, and were for presentations given at recent Goldschmidt Conference meetings, which is one of the premier annual conferences in geochemistry. These abstracts were included in our review, because they are published (and accessible) in a prestigious journal and represent studies of ongoing or recently completed studies in the geochemistry of $\mathrm{CO}_{2}$ sequestration.

Note that Table 5.4 includes two studies related to the carbonate geochemistry of $\mathrm{CO}_{2}$ sequestration as affected by biotic reactions involving microorganisms. Although only two studies were identified (both published as abstracts from Goldschmidt Conferences), both biotic and abiotic processes studies should be considered with respect to thermodynamic data needs associated with modeling geochemical reactions associated with $\mathrm{CO}_{2}$ sequestration. 
Table 5.1. Computer Modeling Simulation Studies of the Carbonate Reactions Associated with $\mathrm{CO}_{2} \mathrm{Sequestration}$

\begin{tabular}{|c|c|c|}
\hline Reference & Sites or Conditions Studied & $\begin{array}{l}\text { Computer } \\
\text { Code(s) Used for Geochemical } \\
\text { Reaction Modeling }\end{array}$ \\
\hline \multirow[t]{2}{*}{ Allen et al. (2005) } & $\begin{array}{l}\mathrm{CO}_{2} \text { gas and calcite solubility in } \mathrm{NaCl} \text { fluid; fluid-rock interactions in a natural brine } \\
\text { contacting a fictitious sandstone (quartz, K-feldspar, albite, and kaolinite) }\end{array}$ & $\begin{array}{l}\text { Geochemist's Workbench, } \\
\text { PHREEQC, and } \mathrm{CO}_{2} \text { solubility } \\
\text { models of Enick and Klara (1990) } \\
\text { and Duan and Sun (2003) }\end{array}$ \\
\hline & \multicolumn{2}{|c|}{ Title of Reference: Modeling Carbon Dioxide Sequestration in Saline Aquifers: Significance of Elevated Pressures and Salinities } \\
\hline \multirow[b]{2}{*}{ André et al. (2007) } & Dogger Aquifer (carbonate reservoir), Paris Basin, France & TOUGHREAC; SCALE2000 \\
\hline & \multicolumn{2}{|c|}{$\begin{array}{l}\text { Title of Reference: Numerical Modeling of Fluid-Rock Chemical Interactions at the Supercritical } \mathrm{CO}_{2} \text {-Liquid Interface during } \\
\mathrm{CO}_{2} \text { Injection into a Carbonate Reservoir, the Dogger Aquifer (Paris Basin, France) }\end{array}$} \\
\hline \multirow[b]{2}{*}{ Audigane et al. (2007) } & Utsira formation in Sleipner West natural gas field, North Sea & TOUGHREACT \\
\hline & \multicolumn{2}{|c|}{$\begin{array}{l}\text { Title of Reference: Two-Dimensional Reactive Transport Modeling of } \mathrm{CO}_{2} \text { Injection in a Saline Aquifer at the Sleipner Site, } \\
\text { North Sea }\end{array}$} \\
\hline \multirow{2}{*}{$\begin{array}{l}\text { Bachu and Adams } \\
\text { (2003) }\end{array}$} & Viking aquifer; Alberta Basin; Canada & SOLMINDB; SOLMINEQ \\
\hline & \multicolumn{2}{|c|}{$\begin{array}{l}\text { Title of Reference: Sequestration of } \mathrm{CO}_{2} \text { in Geological Media in Response to Climate Change: Capacity of Deep Saline Aquifers } \\
\text { to Sequester } \mathrm{CO}_{2} \text { in Solution }\end{array}$} \\
\hline \multirow[b]{2}{*}{ Brosse et al. (2005) } & Limestone made of pure calcite & DIAPHORE \\
\hline & \multicolumn{2}{|c|}{$\begin{array}{l}\text { Title of Reference: Modeling Fluid-Rock Interaction Induced by the Percolation of } \mathrm{CO}_{2} \text {-Enriched Solutions in Core Samples: The } \\
\text { Role of Reactive Surface Area }\end{array}$} \\
\hline \multirow{2}{*}{$\begin{array}{l}\text { Cantucci et al. (2009a) } \\
\text { (abstract only) }\end{array}$} & Saline reservoirs relative to sites in Italy & TOUGHREACT \\
\hline & \multicolumn{2}{|c|}{ Title of Reference: Overview of the Geochemical Modeling on $\mathrm{CO}_{2}$ Capture and Storage in Italian Feasibility Studies } \\
\hline \multirow{2}{*}{ Cantucci et al. (2009b) } & $\mathrm{CO}_{2}$ injection enhancing oil recovery project at Weyburn Oil Field in Saskatchewan, Canada & PHREEQC \\
\hline & \multicolumn{2}{|c|}{ Title of Reference: Geochemical Modeling of $\mathrm{CO}_{2}$ Storage in Deep Reservoirs: The Weyburn Project (Canada) Case Study } \\
\hline \multirow{2}{*}{$\begin{array}{l}\text { Carey and Lichtner } \\
(2007)\end{array}$} & $\begin{array}{l}\text { Carbonation of oilwell cement; SACROC Unit reservoir (continuous } \mathrm{CO}_{2} \text {-flooding operation) } \\
\text { (see Carey et al. [2007] in Table 5.2) in the Cisco and Canyon formations with the Wolfcamp } \\
\text { shale forming the caprock, in the Permian Basin in West Texas, United States }\end{array}$ & FLOTRAN \\
\hline & \multicolumn{2}{|c|}{$\begin{array}{l}\text { Title of Reference: Calcium Silicate Hydrate (C-S-H) Solid Solution Model Applied to Cement Degradation Using the Continuum } \\
\text { Reactive Transport Model FLOTRAN }\end{array}$} \\
\hline
\end{tabular}


Table 5.1. (contd)

\begin{tabular}{|c|c|c|}
\hline Reference & Sites or Conditions Studied & $\begin{array}{c}\text { Computer } \\
\text { Code(s) Used for Geochemical } \\
\text { Reaction Modeling }\end{array}$ \\
\hline \multirow[b]{2}{*}{ Cipolli et al. (2004) } & Serpentinite aquifers such as serpentinites of the Gruppo di Voltri, Genova, Italy & EQ3/EQ6 \\
\hline & \multicolumn{2}{|c|}{$\begin{array}{l}\text { Title of Reference: Geochemistry of High-pH Waters from Serpentinites of the Gruppo di Voltri (Genova, Italy) and Reaction } \\
\text { Path Modeling of } \mathrm{CO}_{2} \text { Sequestration in Serpentinite Aquifers }\end{array}$} \\
\hline \multirow{2}{*}{$\begin{array}{l}\text { Ennis-King and } \\
\text { Paterson (2007) }\end{array}$} & Calcite/anorthite-cemented sandstone and a siliciclastic sandstone & TOUGHREACT \\
\hline & \multicolumn{2}{|c|}{$\begin{array}{l}\text { Title of Reference: Coupling of Geochemical Reactions and Convective Mixing in the Long-Term Geological Storage of Carbon } \\
\text { Dioxide }\end{array}$} \\
\hline \multirow{2}{*}{$\begin{array}{l}\text { Flukiger and Bernard } \\
(2009)\end{array}$} & Dissolution of a limestone that is assumed to be pure calcite & Not named \\
\hline & \multicolumn{2}{|c|}{$\begin{array}{l}\text { Title of Reference: A New Numerical Model for Pore Scale Dissolution of Calcite due to } \mathrm{CO}_{2} \text { Saturated Water Flow in 3D } \\
\text { Realistic Geometry: Principles and First Results }\end{array}$} \\
\hline \multirow{2}{*}{ Gaus et al. (2005) } & Nordland shale cap rock at Sleipner, North Sea & PHREEQC \\
\hline & \multicolumn{2}{|c|}{ Title of Reference: Reactive Transport Modelling of the Impact of $\mathrm{CO}_{2}$ Injection on the Clayey Cap Rock at Sleipner (North Sea) } \\
\hline \multirow[b]{2}{*}{ Gherardi et al. (2007) } & Caprock consisting of carbonate-rich shale & TOUGH-REACT \\
\hline & \multicolumn{2}{|c|}{$\begin{array}{l}\text { Title of Reference: Numerical Modeling of Self-Limiting and Self-Enhancing Caprock Alteration Induced by } \mathrm{CO}_{2} \mathrm{Storage} \mathrm{in} \mathrm{a}_{\text {Depleted Gas Reservoir }}\end{array}$} \\
\hline \multirow{2}{*}{ Gunter et al. (2000) } & Deep siliciclastic and carbonate aquifers in the Alberta Sedimentary Basin, Canada & PATHARC.94 \\
\hline & \multicolumn{2}{|c|}{ Title of Reference: Aquifer Disposal of Acid Gases: Modelling of Water-Rock Reactions for Trapping of Acid Wastes } \\
\hline \multirow{2}{*}{$\begin{array}{l}\text { Gysi and Stefánsson } \\
(2008)\end{array}$} & Basaltic glass from Stapafee, southwest Iceland & PHREEQC \\
\hline & \multicolumn{2}{|l|}{ Title of Reference: Numerical Modelling of $\mathrm{CO}_{2}$-Water-Basalt Interaction } \\
\hline \multirow{2}{*}{$\begin{array}{l}\text { Hurter et al. (2007) } \\
\text { (abstract only) }\end{array}$} & Northern German Basin & No details \\
\hline & \multicolumn{2}{|l|}{ Title of Reference: Simulations of Dry-Out and Halite Precipitation due to $\mathrm{CO}_{2}$ Injection } \\
\hline \multirow{2}{*}{ Izgec et al. (2008b) } & Carbonate formations - Midyat formation, southeast Turkey; and St. Maximim, France & $\begin{array}{l}\text { STARS, also see Izgec et al. } \\
\text { (2008a) in Table } 5.4\end{array}$ \\
\hline & \multicolumn{2}{|c|}{$\begin{array}{l}\text { Title of Reference: } \mathrm{CO}_{2} \text { Injection into Saline Carbonate Aquifer Formations II: Comparison of Numerical Simulations to } \\
\text { Experiments }\end{array}$} \\
\hline
\end{tabular}


Table 5.1. (contd)

\begin{tabular}{|c|c|c|}
\hline Reference & Sites or Conditions Studied & $\begin{array}{l}\text { Computer } \\
\text { Code(s) Used for Geochemical } \\
\text { Reaction Modeling }\end{array}$ \\
\hline \multirow[b]{2}{*}{ Johnson et al. (2001) } & Shale-capped sandstone aquifer at Sleipner facility, North Sea & NUFT \\
\hline & \multicolumn{2}{|c|}{$\begin{array}{l}\text { Title of Reference: Reactive Transport Modeling of Geologic } \mathrm{CO}_{2} \text { Sequestration in Saline Aquifers: The Influence of Intra- } \\
\text { Aquifer Shales and the Relative Effectiveness of Structural, Solubility, and Mineral Trapping During Prograde and Retrograde } \\
\text { Sequestration }\end{array}$} \\
\hline \multirow[b]{2}{*}{ Johnson et al. (2004) } & Shale-capped sandstone aquifer at Sleipner facility, North Sea & NUFT \\
\hline & \multicolumn{2}{|c|}{$\begin{array}{l}\text { Title of Reference: Reactive Transport Modelling of } \mathrm{CO}_{2} \text { Storage in Saline Aquifers to Elucidate Fundamental Processes, } \\
\text { Trapping Mechanisms, and Sequestration Partitioning }\end{array}$} \\
\hline \multirow[b]{2}{*}{ Kervévan et al. (2005) } & Carbonated reservoir with brine composition from Smackover site, Arkansas, United States & SCALE2000; PHREEQC \\
\hline & \multicolumn{2}{|c|}{$\begin{array}{l}\text { Title of Reference: Improvement of the Calculation Accuracy of Acid Gas Solubility in Deep Reservoir Brines: Application to the } \\
\text { Geological Storage of } \mathrm{CO}_{2}\end{array}$} \\
\hline \multirow[b]{2}{*}{ Knauss et al. (2005) } & Frio Formation pilot site, Texas, United States & CRUNCH \\
\hline & \multicolumn{2}{|c|}{$\begin{array}{l}\text { Title of Reference: Evaluation of the Impact of } \mathrm{CO}_{2} \text {, Co-contaminant Gas, Aqueous Fluid and Reservoir Rock Interactions on the } \\
\text { Geologic Sequestration of } \mathrm{CO}_{2}\end{array}$} \\
\hline \multirow{2}{*}{$\begin{array}{l}\text { Kühn and Clauser } \\
\text { (2007) (abstract only) }\end{array}$} & Sandstone aquifers in Germany & No details of modeling \\
\hline & \multicolumn{2}{|c|}{ Title of Reference: Reactive Transport Simulation of Mineral Trapping of $\mathrm{CO}_{2}$ in Operated Geothermal Aquifers } \\
\hline \multirow[t]{2}{*}{ Lagneau et al. (2005) } & $\begin{array}{l}\text { Carbonate aquifer of the Dogger, Paris Basin in France; sandstone aquifer of the Bunter in the } \\
\text { North Sea }\end{array}$ & Chess \\
\hline & \multicolumn{2}{|l|}{ Title of Reference: Reactive Transport Modelling of $\mathrm{CO}_{2}$ Sequestration in Saline Aquifers } \\
\hline \multirow{2}{*}{$\begin{array}{l}\text { Mito and Nakagawa } \\
(2009) \\
\text { (abstract only) }\end{array}$} & Deep saline aquifer at pilot-scale $\mathrm{CO}_{2}$ injection test at Nagaoka site in Japan & PHREEQC \\
\hline & \multicolumn{2}{|l|}{ Title of Reference: Influence of Formation Water Composition on Mineral Trapping of $\mathrm{CO}_{2}$} \\
\hline \multirow{2}{*}{ Ozah et al. (2005) } & Deep saline aquifer typical of U.S. Gulf Coast & GEM-GHG \\
\hline & \multicolumn{2}{|c|}{ Title of Reference: Numerical Simulation of the Storage of Pure $\mathrm{CO}_{2}$ and $\mathrm{CO}_{2}-\mathrm{H}_{2} \mathrm{~S}$ Gas Mixtures in Deep Saline Aquifers } \\
\hline \multirow{2}{*}{$\begin{array}{l}\text { Palandri and Kharaka } \\
(2005)\end{array}$} & $\mathrm{Fe}^{\mathrm{III}}$-bearing quartz arenite (synthetic redbed) & CHILLER; GAMSPATH \\
\hline & \multicolumn{2}{|c|}{$\begin{array}{l}\text { Title of Reference: Ferric Iron-Bearing Sediments as a Mineral Trap for } \mathrm{CO}_{2} \text { Sequestration: Iron Reduction Using Sulfur-Bearing } \\
\text { Waste Gas }\end{array}$} \\
\hline
\end{tabular}


Table 5.1. (contd)

\begin{tabular}{|c|c|c|}
\hline Reference & Sites or Conditions Studied & $\begin{array}{c}\text { Computer } \\
\text { Code(s) Used for Geochemical } \\
\text { Reaction Modeling }\end{array}$ \\
\hline \multirow{2}{*}{$\begin{array}{l}\text { Pruess et al. (2004); } \\
\text { Pruess et al. (2002) }\end{array}$} & $\begin{array}{l}\text { Problem } 5 \text { in Pruess et al. (2004) addresses geochemical effects of } \mathrm{CO}_{2} \text { injection into } \\
\text { glauconitic sandstone }\end{array}$ & $\begin{array}{l}\text { TOUGHREAC, FLOTRAN, } \\
\text { CHEM-TOUGH2 }\end{array}$ \\
\hline & \multicolumn{2}{|c|}{ Title of Reference: Code Intercomparison Builds Confidence in Numerical Simulation Models for Geologic Disposal of $\mathrm{CO}_{2}$} \\
\hline \multirow{2}{*}{$\begin{array}{l}\text { Saylor and Zerai } \\
\text { (2004) }\end{array}$} & Rose Run Sandstone, Ohio & Geochemist's Workbench \\
\hline & \multicolumn{2}{|l|}{ Title of Reference: Injection and Trapping of Carbon Dioxide in Deep Saline Aquifers } \\
\hline \multirow{2}{*}{ White et al. (2005) } & White Rim Sandstone, Colorado Plateau, Utah & ChemTOUGH \\
\hline & \multicolumn{2}{|c|}{ Title of Reference: Simulation of Reactive Transport of Injected $\mathrm{CO}_{2}$ on the Colorado Plateau, Utah, USA } \\
\hline \multirow[b]{2}{*}{ Xu et al. (2003) } & Gulf Coast sediment of the United States & TOUGHREACT \\
\hline & \multicolumn{2}{|c|}{$\begin{array}{l}\text { Title of Reference: Reactive Geochemical Transport Simulation to Study Mineral Trapping for } \mathrm{CO}_{2} \text { Disposal in Deep Arenaceous } \\
\text { Formations }\end{array}$} \\
\hline \multirow[t]{2}{*}{ Xu et al. (2004) } & $\begin{array}{l}\text { Glauconitic sandstone aquifer from the Alberta Sedimentary Basin, Gulf Coast sediment of } \\
\text { the United States, dunite (monomineralic rock consisting essentially of olivine) }\end{array}$ & TOUGHREACT \\
\hline & \multicolumn{2}{|l|}{ Title of Reference: Numerical Simulation of $\mathrm{CO}_{2}$ Disposal by Mineral Trapping in Deep Aquifers } \\
\hline \multirow{2}{*}{ Xu et al. (2005) } & Sandstone and shale layers representative of Texas Gulf Coast sediment of the United States & TOUGHREACT \\
\hline & \multicolumn{2}{|l|}{ Title of Reference: Mineral Sequestration of Carbon Dioxide in a Sandstone-Shale System } \\
\hline \multirow[b]{2}{*}{ Xu et al. (2006) } & Gulf Coast sediment of the United States & TOUGHREACT \\
\hline & \multicolumn{2}{|c|}{$\begin{array}{l}\text { Title of Reference: TOUGHREACT - A Simulation Program for Non-Isothermal Multiphase Reactive Geochemical Transport in } \\
\text { Variably Saturated Geologic Media: Applications to Geothermal Injectivity and } \mathrm{CO}_{2} \text { Geological Sequestration }\end{array}$} \\
\hline \multirow{2}{*}{ Xu et al. (2007) } & $\begin{array}{l}\text { Co-injection of } \mathrm{H}_{2} \mathrm{~S} \text { or } \mathrm{SO}_{2} \text { with } \mathrm{CO}_{2} \text { into an arkose formation as typically encountered in } \\
\text { Gulf Coast aquifers of the United States }\end{array}$ & TOUGHREACT \\
\hline & \multicolumn{2}{|c|}{ Title of Reference: Numerical Modeling of Injection and Mineral Trapping of $\mathrm{CO}_{2}$ with $\mathrm{H}_{2} \mathrm{~S}$ and $\mathrm{SO}_{2}$ in a Sandstone Formation } \\
\hline \multirow{2}{*}{ Zerai et al. (2006) } & Rose Run Sandstone, Ohio & Geochemist's Workbench \\
\hline & \multicolumn{2}{|c|}{ Title of Reference: Computer Simulation of $\mathrm{CO}_{2}$ Trapped through Mineral Precipitation in the Rose Run Sandstone, Ohio } \\
\hline
\end{tabular}


Table 5.1. (contd)

\begin{tabular}{|c|c|c|}
\hline Reference & Sites or Conditions Studied & $\begin{array}{l}\text { Computer } \\
\text { Code(s) Used for Geochemical } \\
\text { Reaction Modeling }\end{array}$ \\
\hline \multirow[t]{2}{*}{ Zhang et al. (2009) } & $\begin{array}{l}\text { Songliao Basin, China - proposed injection formation is saline sandstone formation (quartz, } \\
\text { kaolinite, illite, chlorite, calcite, plagioclase, and lesser amounts of K-feldspar, zeolite, pyrite, } \\
\text { and anhydrite) }\end{array}$ & TOUGHREACT \\
\hline & \multicolumn{2}{|c|}{$\begin{array}{l}\text { Title of Reference: Long-Term Variations of } \mathrm{CO}_{2} \text { Trapped in Different Mechanisms in Deep Saline Formations: A Case Study of } \\
\text { the Songliao Basin, China }\end{array}$} \\
\hline \multirow{2}{*}{$\begin{array}{l}\text { Zwingmann et al. } \\
(2005)\end{array}$} & $\begin{array}{l}\text { Modeling done prior to } \mathrm{CO}_{2} \text { injection; Haizume Formation, Niigata Basin, Japan - injection } \\
\text { formation is sedimentary formation (quartz, plagioclase, feldspar, pyroxene, and clays) } \\
\text { covered by a mudstone seal }\end{array}$ & EQ3/EQ6 \\
\hline & \multicolumn{2}{|c|}{$\begin{array}{l}\text { Title of Reference: Preinjection Characterisation and Evaluation of } \mathrm{CO}_{2} \text { Sequestration Potential in the Haizume Formation, } \\
\text { Niigata Basin, Japan. Geochemical Modelling of Water-Minerals- } \mathrm{CO}_{2} \text { Interaction }\end{array}$} \\
\hline
\end{tabular}


Table 5.2. Studies of Mineral Characterization Data and/or Geochemical Analyses at $\mathrm{CO}_{2}$ Field Injection Sites

\begin{tabular}{|c|c|c|c|c|}
\hline Reference & Site(s) Studied & $\begin{array}{l}\text { Carbonate Minerals and } \\
\text { Reactions Physically Observed }\end{array}$ & $\begin{array}{c}\text { Was } \\
\text { Geochemical } \\
\text { Reaction } \\
\text { Modeling } \\
\text { Used? }\end{array}$ & $\begin{array}{l}\text { Computer } \\
\text { Codes Used for } \\
\text { Geochemical } \\
\text { Reaction } \\
\text { Modeling }\end{array}$ \\
\hline \multirow{2}{*}{$\begin{array}{l}\text { Alfredsson and } \\
\text { Gislason (2009) } \\
\text { (abstract only) }\end{array}$} & $\begin{array}{l}\text { Basaltic Rock: Chemistry of the Rocks and Waters at the } \\
\text { Injection Site, Hellisheidi, southwestern Iceland }\end{array}$ & None discussed & Yes & PHREEQC \\
\hline & \multicolumn{4}{|c|}{$\begin{array}{l}\text { Title of Reference: CarbFix - } \mathrm{CO}_{2} \text { Sequestration in Basaltic Rock: Chemistry of the Rocks and Waters at the Injection Site, } \\
\text { Hellisheidi, SW-Iceland }\end{array}$} \\
\hline \multirow{2}{*}{ Assayag et al. (2009) } & $\begin{array}{l}\text { Lamont-Doherty Earth observatory test site (New York, } \\
\text { United States) - injection at contract between dolerites and } \\
\text { underlying Newark Basin metamorphosed sediments }\end{array}$ & None discussed & $\begin{array}{c}\text { None } \\
\text { discussed }\end{array}$ & \\
\hline & \multicolumn{4}{|c|}{$\begin{array}{l}\text { Title of Reference: Water-Rock Interactions during a } \mathrm{CO}_{2} \text { Injection Field-Test: Implications on Host Rock Dissolution and } \\
\text { Alteration Effects }\end{array}$} \\
\hline \multirow[t]{2}{*}{$\begin{array}{l}\text { Assayag et al. (2008) } \\
\text { (abstract only) }\end{array}$} & $\begin{array}{l}\text { Lamont-Doherty Earth observatory test site (New York, } \\
\text { United States) - injection at contract between dolerites and } \\
\text { underlying Newark Basin metamorphosed sediments }\end{array}$ & None discussed & $\begin{array}{c}\text { None } \\
\text { discussed }\end{array}$ & \\
\hline & \multicolumn{4}{|c|}{ Title of Reference: $\mathrm{CO}_{2}$ Ionic Trapping by Water-Rock Interactions during a Push-Pull Test in a Basaltic-Metasedimentary Aquifer } \\
\hline \multirow[t]{2}{*}{ Carey et al. (2007) } & $\begin{array}{l}\text { Carbonation of oilwell cement; SACROC Unit reservoir } \\
\text { (continuous } \mathrm{CO}_{2} \text {-flooding operation) (see Carey and } \\
\text { Lichtner [2007] in Table 5.1) in the Cisco and Canyon } \\
\text { formations with the Wolfcamp shale forming the caprock, } \\
\text { in the Permian Basin in West Texas, United States }\end{array}$ & $\begin{array}{l}\text { Limestone reservoir is mostly } \\
\text { calcite with lesser ankerite; } \\
\text { carbonated cement contains } \\
\text { calcite and aragonite; vein- } \\
\text { fillings at cement-casing } \\
\text { interface contain calcite and } \\
\text { aragonite; carbonates at cement- } \\
\text { shale interface include calcite, } \\
\text { aragonite, and vaterite (and } \\
\text { possibly dawsonite); matrix of } \\
\text { shale fragment zone contains the } \\
\text { carbonates calcite, aragonite, } \\
\text { and dolomite }\end{array}$ & Yes & FLOTRAN \\
\hline & \multicolumn{4}{|c|}{$\begin{array}{l}\text { Title of Reference: Analysis and Performance of Oil Well Cement with } 30 \text { Years of } \mathrm{CO}_{2} \text { Exposure from the SACROC Unit, West } \\
\text { Texas, USA }\end{array}$} \\
\hline
\end{tabular}


Table 5.2. (contd)

\begin{tabular}{|c|c|c|c|c|}
\hline Reference & Site(s) Studied & $\begin{array}{l}\text { Carbonate Minerals and } \\
\text { Reactions Physically Observed }\end{array}$ & $\begin{array}{c}\text { Was } \\
\text { Geochemical } \\
\text { Reaction } \\
\text { Modeling } \\
\text { Used? }\end{array}$ & $\begin{array}{c}\text { Computer } \\
\text { Codes Used for } \\
\text { Geochemical } \\
\text { Reaction } \\
\text { Modeling }\end{array}$ \\
\hline \multirow[b]{2}{*}{ Emberley et al. (2004) } & Weyburn Oil Field, Saskatchewan, Canada & None discussed & Yes & Not identified \\
\hline & \multicolumn{4}{|c|}{$\begin{array}{l}\text { Title of Reference: Geochemical Monitoring of Fluid-Rock Interaction and } \mathrm{CO}_{2} \text { Storage at the Weyburn } \mathrm{CO}_{2} \text {-Injection Enhanced } \\
\text { Oil Recovery Site, Saskatchewan, Canada }\end{array}$} \\
\hline \multirow[b]{2}{*}{ Emberley et al. (2005) } & Weyburn Oil Field, Saskatchewan, Canada & None discussed & Yes & SOLMINEQ.88 \\
\hline & \multicolumn{4}{|c|}{$\begin{array}{l}\text { Title of Reference: Monitoring of Fluid-Rock Interaction and } \mathrm{CO}_{2} \text { Storage Through Produced Fluid Sampling at the Weyburn } \\
\mathrm{CO}_{2} \text {-Injection Enhanced Oil Recovery Site, Saskatchewan, Canada }\end{array}$} \\
\hline \multirow{2}{*}{$\begin{array}{l}\text { Gislason et al. (2009) } \\
\text { (abstract only) }\end{array}$} & $\begin{array}{l}\text { Overview - field, laboratory and modeling studies of basalt } \\
\text { at the Hellisheidi, Injection Site in southwestern Iceland }\end{array}$ & None discussed & Yes & Not identified \\
\hline & \multicolumn{4}{|c|}{ Title of Reference: The Carbfix Project: Mineral $\mathrm{CO}_{2}$ Sequestration into Basalt } \\
\hline \multirow[t]{2}{*}{ Gislason et al. (2010) } & $\begin{array}{l}\text { Overview - field, laboratory and modeling studies of basalt } \\
\text { at the Hellisheidi, Injection Site in southwestern Iceland }\end{array}$ & None discussed & Yes & Not identified \\
\hline & \multicolumn{4}{|c|}{ Title of Reference: Mineral Sequestration of Carbon Dioxide in Basalt: A Pre-injection Overview of the Carbfix Project } \\
\hline \multirow[t]{2}{*}{ Goldberg et al. (2008) } & $\begin{array}{l}\text { Review of the feasibility of } \mathrm{CO}_{2} \text { sequestration in deep sea } \\
\text { basalts such as Juan de Fuca ridge }\end{array}$ & None discussed & $\begin{array}{c}\text { None } \\
\text { discussed }\end{array}$ & \\
\hline & \multicolumn{4}{|c|}{ Title of Reference: Carbon Dioxide Sequestration in Deep-Sea Basalt } \\
\hline \multirow{2}{*}{ Kharaka et al. (2006a) } & $\begin{array}{l}\text { Sandstone sections of the Frio Formation pilot site, Texas, } \\
\text { United States }\end{array}$ & None discussed & Yes & SOLMINEQ \\
\hline & \multicolumn{4}{|c|}{$\begin{array}{l}\text { Title of Reference: Gas-Water-Rock Interactions in Frio Formation Following } \mathrm{CO}_{2} \text { Injection: Implications for the Storage of } \\
\text { Greenhouse Gases in Sedimentary Basins }\end{array}$} \\
\hline \multirow[t]{2}{*}{ Kharaka et al.(2006b) } & $\begin{array}{l}\text { Sandstone sections of the Frio Formation pilot site, Texas, } \\
\text { United States }\end{array}$ & None discussed & Yes & SOLMINEQ \\
\hline & \multicolumn{4}{|c|}{ Title of Reference: Gas-Water-Rock Interactions in Sedimentary Basins: $\mathrm{CO}_{2}$ Sequestration in the Frio Formation, Texas, USA } \\
\hline \multirow{2}{*}{$\begin{array}{l}\text { Kharaka et al. (2007) } \\
\text { (abstract only) }\end{array}$} & $\begin{array}{l}\text { Sandstone sections of the Frio Formation pilot site, Texas, } \\
\text { United States }\end{array}$ & None discussed & Yes & Not identified \\
\hline & \multicolumn{4}{|c|}{$\begin{array}{l}\text { Title of Reference: Potential Environmental Issues of } \mathrm{CO}_{2} \text { Storage in Saline Aquifers: Geochemical Results from the Frio Brine } \\
\text { Pilot Tests, Texas, USA }\end{array}$} \\
\hline
\end{tabular}


Table 5.2. (contd)

\begin{tabular}{|c|c|c|c|c|}
\hline Reference & Site(s) Studied & $\begin{array}{c}\text { Carbonate Minerals and } \\
\text { Reactions Physically Observed }\end{array}$ & $\begin{array}{l}\text { Was } \\
\text { Geochemical } \\
\text { Reaction } \\
\text { Modeling } \\
\text { Used? }\end{array}$ & $\begin{array}{l}\text { Computer } \\
\text { Codes Used for } \\
\text { Geochemical } \\
\text { Reaction } \\
\text { Modeling }\end{array}$ \\
\hline \multirow{2}{*}{ Machel (2005) } & Nisku Q-Pool in Alberta, Canada & $\begin{array}{l}\text { Formation has dolomite with } \\
\text { minor amounts of calcite }\end{array}$ & $\begin{array}{c}\text { None } \\
\text { discussed }\end{array}$ & \\
\hline & \multicolumn{4}{|c|}{$\begin{array}{l}\text { Title of Reference: Geological and Hydrogeological Evaluation of the Nisku Q-Pool in Alberta, Canada, for } \mathrm{H}_{2} \mathrm{~S} \text { and/or } \mathrm{CO}_{2} \\
\text { Storage }\end{array}$} \\
\hline \multirow{2}{*}{ Mani et al. (2008) } & $\begin{array}{l}\text { Review of the feasibility of } \mathrm{CO}_{2} \text { sequestration in ultramafic } \\
\text { rocks of Kolar and Chitradurga greenstone belts in India }\end{array}$ & None discussed & $\begin{array}{c}\text { None } \\
\text { discussed }\end{array}$ & \\
\hline & \multicolumn{4}{|c|}{$\begin{array}{l}\text { Title of Reference: Assessment of Carbon Dioxide Sequestration Potential of Ultramafic Rocks in the Greenstone Belts of Southern } \\
\text { India }\end{array}$} \\
\hline \multirow[t]{2}{*}{ Mito et al. (2008) } & $\begin{array}{l}\text { Sandstone bed (Minami-Nagaoka gas field) of the Haizume } \\
\text { Formation at Nagaoka, Japan }\end{array}$ & $\begin{array}{l}\text { Calcite observed in original } \\
\text { reservoir rock; characterization } \\
\text { of specific carbonate mineral } \\
\text { products were not identified }\end{array}$ & Yes & $\begin{array}{l}\text { PHREEQC, } \\
\text { ChemTOUGH }\end{array}$ \\
\hline & \multicolumn{4}{|c|}{ Title of Reference: Case Study of Geochemical Reactions at the Nagaoka $\mathrm{CO}_{2}$ Injection Site, Japan } \\
\hline \multirow[t]{2}{*}{ Raistrick et al. (2006) } & $\begin{array}{l}\text { Weyburn } \mathrm{CO}_{2} \text { Monitoring and Storage Project, } \\
\text { Saskatchewan, Canada }\end{array}$ & None discussed & Yes & SOLMINEQ.88 \\
\hline & \multicolumn{4}{|c|}{ Title of Reference: Using Chemical and Isotopic Data to Quantify Ionic Trapping of Injected Carbon Dioxide in Oil Field Brines } \\
\hline \multirow{2}{*}{$\begin{array}{l}\text { Ueda et al. (2007) } \\
\text { (abstract only) }\end{array}$} & Ogachi HDR (Hot-Dry Rock) Site, northeast Japan & None discussed & $\begin{array}{c}\text { None } \\
\text { discussed }\end{array}$ & \\
\hline & \multicolumn{4}{|c|}{ Title of Reference: Preliminary Geochemical Results on the $\mathrm{CO}_{2}$ Georeactor Sequestration Test at the Ogachi HDR Site } \\
\hline \multirow{2}{*}{$\begin{array}{l}\text { Ueda et al. (2008) } \\
\text { (abstract only) }\end{array}$} & Ogachi HDR (Hot-Dry Rock) Site, northeast Japan & $\begin{array}{l}\text { Precipitation inferred from } \\
\text { in situ analyses }\end{array}$ & $\begin{array}{c}\text { None } \\
\text { discussed }\end{array}$ & \\
\hline & \multicolumn{4}{|c|}{ Title of Reference: Geochemical Results on the $\mathrm{CO}_{2}$ Georeactor Sequestration Tests at the Ogachi Hot Dry Rock Site, NE Japan } \\
\hline
\end{tabular}


Table 5.2. (contd)

\begin{tabular}{|c|c|c|c|c|}
\hline Reference & Site(s) Studied & $\begin{array}{c}\text { Carbonate Minerals and } \\
\text { Reactions Physically Observed }\end{array}$ & $\begin{array}{l}\text { Was } \\
\text { Geochemical } \\
\text { Reaction } \\
\text { Modeling } \\
\text { Used? }\end{array}$ & $\begin{array}{l}\text { Computer } \\
\text { Codes Used for } \\
\text { Geochemical } \\
\text { Reaction } \\
\text { Modeling }\end{array}$ \\
\hline \multirow{2}{*}{$\begin{array}{l}\text { Ueda et al. (2009) } \\
\text { (abstract only) }\end{array}$} & Ogachi HDR (Hot-Dry Rock) Site, northeast Japan & $\begin{array}{l}\text { Calcite precipitation inferred } \\
\text { from in situ analyses }\end{array}$ & $\begin{array}{c}\text { None } \\
\text { discussed }\end{array}$ & \\
\hline & \multicolumn{4}{|c|}{ Title of Reference: Geochemical Monitoring of Calcite Precipitation during $\mathrm{CO}_{2}$ Injection into the Ogachi Hot Dry Rock Site } \\
\hline \multirow[t]{2}{*}{ Westrich et al. (2002) } & $\begin{array}{l}\text { Depleted oil reservoir in the Queen Formation sandstone in } \\
\text { the West Pearl Queen Field in southeast New Mexico } \\
\text { Overview of studies and proposed measurements - field } \\
\text { injection site with planned modeling, laboratory tests, and } \\
\text { other activities }\end{array}$ & None discussed & Yes & Not identified \\
\hline & \multicolumn{4}{|c|}{ Title of Reference: Sequestration of $\mathrm{CO}_{2}$ in a Depleted Oil Reservoir: An Overview } \\
\hline
\end{tabular}


Table 5.3. Studies of Mineral Characterization Data and/or Geochemical Analyses from Sites Considered Possible Natural Analogues for Carbonate Processes Associated $\mathrm{CO}_{2}$ Injection and Sequestration

\begin{tabular}{|c|c|c|c|c|c|}
\hline Reference & Study & Sites Studied & $\begin{array}{c}\text { Carbonate Minerals and } \\
\text { Reactions Physically } \\
\text { Observed }\end{array}$ & $\begin{array}{l}\text { Was } \\
\text { Geochemical } \\
\text { Reaction } \\
\text { Modeling } \\
\text { Used? }\end{array}$ & $\begin{array}{c}\text { Computer } \\
\text { Codes Used for } \\
\text { Geochemical } \\
\text { Reaction } \\
\text { Modeling }\end{array}$ \\
\hline \multirow[t]{2}{*}{$\begin{array}{l}\text { Baines and Worden } \\
\text { (2004) }\end{array}$} & Natural analogue & $\begin{array}{l}\text { Carbonate reservoirs - Blue } \\
\text { Whale and Dolphin in the } \\
\text { Da Nang Basin offshore } \\
\text { Vietnam; quartzose sandstone } \\
\text { reservoirs - Miller and Magnus } \\
\text { fields in the UK North Sea; red } \\
\text { bed sandstone reservoirs - } \\
\text { Bravo Dome in New Mexico } \\
\text { and Vert le Grand in Paris } \\
\text { Basin. }\end{array}$ & $\begin{array}{l}\text { Blue Whale and Dolphin- } \\
\text { have marine carbonate cements, } \\
\text { early diagenetic vugs have } \\
\text { ferroan (Fe-bearing) calcite, } \\
\text { calcite late post-vug filling and } \\
\text { late diagenetic dolomite cement } \\
\text { at Dolphin. Miller and } \\
\text { Magnus - early diagenetic } \\
\text { assemblages include non- and } \\
\text { weakly ferroan calcite cement; } \\
\text { late stages include ferroan } \\
\text { dolomite or ankerite cement; } \\
\text { ferroan dolomite followed by } \\
\text { non-ferroan calcite at Miller. } \\
\text { Bravo Dome - final cements } \\
\text { have ferroan dolomite and } \\
\text { siderite. Vert le Grand - } \\
\text { carbonate cements include } \\
\text { ferroan dolomite, saddle } \\
\text { dolomite, and ferroan calcite. }\end{array}$ & Yes & $\begin{array}{l}\text { Geochemist's } \\
\text { Workbench }\end{array}$ \\
\hline & \multicolumn{5}{|c|}{ Title of Reference: The Long-Term Fate of $\mathrm{CO}_{2}$ in the Subsurface: Natural Analogues for $\mathrm{CO}_{2}$ Storage } \\
\hline \multirow[t]{2}{*}{$\begin{array}{l}\text { Beinlich et al. (2009) } \\
\text { (abstract only) }\end{array}$} & $\begin{array}{l}\text { Sample } \\
\text { characterization }\end{array}$ & $\begin{array}{l}\text { Serpentinized peridotite clasts in } \\
\text { conglomerate from the Solund } \\
\text { Devonian Basin in southwestern } \\
\text { Norway }\end{array}$ & Calcite & None discussed & \\
\hline & \multicolumn{5}{|c|}{$\begin{array}{l}\text { Title of Reference: } \mathrm{CO}_{2} \text { Sequestration and Extreme } \mathrm{Mg} \text { Leaching in Serpentinized Peridotite Clasts of the Solund Devonian Basin, } \\
\text { SW-Norway }\end{array}$} \\
\hline
\end{tabular}


Table 5.3. (contd)

\begin{tabular}{|c|c|c|c|c|c|}
\hline Reference & Study & Sites Studied & $\begin{array}{c}\text { Carbonate Minerals and } \\
\text { Reactions Physically } \\
\text { Observed }\end{array}$ & $\begin{array}{l}\text { Was } \\
\text { Geochemical } \\
\text { Reaction } \\
\text { Modeling } \\
\text { Used? }\end{array}$ & $\begin{array}{l}\text { Computer } \\
\text { Codes Used for } \\
\text { Geochemical } \\
\text { Reaction } \\
\text { Modeling }\end{array}$ \\
\hline \multirow[t]{2}{*}{ Boschi et al. (2009) } & $\begin{array}{l}\text { Sample } \\
\text { characterization }\end{array}$ & $\begin{array}{l}\text { Magnesite deposits of } \\
\text { Malentrata in Tuscany, Italy, } \\
\text { which were derived from } \\
\text { serpentinite silicification- } \\
\text { carbonation of an ophiolite }\end{array}$ & $\begin{array}{l}\text { Early formation of Fe-poor } \\
\text { magnesite followed by Fe-rich } \\
\text { magnesite and dolomite } \\
\text { cementing the early brecciated } \\
\text { magnesite vein }\end{array}$ & None discussed & \\
\hline & \multicolumn{5}{|c|}{$\begin{array}{l}\text { Title of Reference: Enhanced } \mathrm{CO}_{2} \text {-Mineral Sequestration by Cyclic Hydraulic Fracturing and Si-Rich Fluid Infiltration into } \\
\text { Serpentinites at Malentrata (Tuscany, Italy) }\end{array}$} \\
\hline \multirow{2}{*}{$\begin{array}{l}\text { Bruno et al. (2009) } \\
\text { (abstract only) }\end{array}$} & Review & $\begin{array}{l}\text { Naturally occurring } \mathrm{CO}_{2} \\
\text { abundances in Spain }\end{array}$ & None discussed & Yes & Not identified \\
\hline & \multicolumn{5}{|c|}{ Title of Reference: Trace Element Behaviour in Connection to the Geological Storage of $\mathrm{CO}_{2}$ : Lessons from Natural Analogues } \\
\hline \multirow[t]{2}{*}{$\begin{array}{l}\text { Flaathen and Gislason } \\
\text { (2007) }\end{array}$} & $\begin{array}{l}\text { Natural analogue and } \\
\text { possible field injection } \\
\text { site }\end{array}$ & $\begin{array}{l}\text { Groundwater beneath Mt. Hekla } \\
\text { Volcano, Iceland }\end{array}$ & None discussed & Yes & PHREEQC \\
\hline & \multicolumn{5}{|c|}{ Title of Reference: The Groundwater Beneath Hekla Volcano, Iceland; A Natural Analogue for $\mathrm{CO}_{2}$ Sequestration } \\
\hline \multirow{2}{*}{ Flaathen et al. (2009) } & $\begin{array}{l}\text { Natural analogue and } \\
\text { possible field injection } \\
\text { site }\end{array}$ & $\begin{array}{l}\text { Spring water samples } \\
\text { surrounding Mt. Hekla Volcano, } \\
\text { Iceland }\end{array}$ & None discussed & Yes & PHREEQC \\
\hline & \multicolumn{5}{|c|}{$\begin{array}{l}\text { Title of Reference: Chemical Evolution of the Mt. Hekla, Iceland, Groundwaters: A Natural Analogue for } \mathrm{CO}_{2} \text { Sequestration in } \\
\text { Basaltic Rocks }\end{array}$} \\
\hline \multirow{2}{*}{ Hansen et al. (2005) } & Natural analogue & $\begin{array}{l}\text { Carbonated serpentinite } \\
\text { (listwanite) at Atlin, British } \\
\text { Columbia }\end{array}$ & Magnesite & None discussed & \\
\hline & \multicolumn{5}{|c|}{$\begin{array}{l}\text { Title of Reference: Carbonated Serpentinite (Listwanite) at Atlin, British Columbia: A Geological Analogue to Carbon Dioxide } \\
\text { Sequestration }\end{array}$} \\
\hline
\end{tabular}


Table 5.3. (contd)

\begin{tabular}{|c|c|c|c|c|c|}
\hline Reference & Study & Sites Studied & $\begin{array}{c}\text { Carbonate Minerals and } \\
\text { Reactions Physically } \\
\text { Observed }\end{array}$ & $\begin{array}{c}\text { Was } \\
\text { Geochemical } \\
\text { Reaction } \\
\text { Modeling } \\
\text { Used? }\end{array}$ & $\begin{array}{c}\text { Computer } \\
\text { Codes Used for } \\
\text { Geochemical } \\
\text { Reaction } \\
\text { Modeling }\end{array}$ \\
\hline \multirow[t]{2}{*}{ Haszeldine et al. (2005) } & $\begin{array}{l}\text { Natural analogue } \\
\text { (overview) }\end{array}$ & $\begin{array}{l}\text { Natural examples - United } \\
\text { Kingdom oilfields; natural } \\
\text { exhumed examples - Colorado } \\
\text { Plateau; modern } \mathrm{CO}_{2} \text { leakage up } \\
\text { faults - Salt Wash, Utah and } \\
\text { Springerville-St. Johns, Arizona, } \\
\text { United States; ancient } \mathrm{CO}_{2} \\
\text { paleo-trap - Moab Anticline, } \\
\text { Utah, United States } \\
\end{array}$ & None discussed & No & \\
\hline & \multicolumn{5}{|c|}{$\begin{array}{l}\text { Title of Reference: Natural Geochemical Analogues for Carbon Dioxide Storage in Deep Geological Porous Reservoirs, a United } \\
\text { Kingdom Perspective }\end{array}$} \\
\hline \multirow{2}{*}{$\begin{array}{l}\text { Hövelmann and } \\
\text { Austrheim (2009) } \\
\text { (abstract only) }\end{array}$} & $\begin{array}{l}\text { Sample } \\
\text { characterization }\end{array}$ & $\begin{array}{l}\text { Peridotite clasts from Solund } \\
\text { and Fensfjorden Devonian } \\
\text { basins of southwestern Norway }\end{array}$ & Calcite & None discussed & \\
\hline & \multicolumn{5}{|c|}{ Title of Reference: Guidelines for Experiments on $\mathrm{CO}_{2}$ Sequestration in Peridotites based on a Natural Example } \\
\hline \multirow{2}{*}{$\begin{array}{l}\text { Matter and Kelemen } \\
\text { (2009) } \\
\text { (abstract only) }\end{array}$} & $\begin{array}{l}\text { Sample } \\
\text { characterization }\end{array}$ & $\begin{array}{l}\text { Natural carbonation of peridotite } \\
\text { from ophiolite in Oman }\end{array}$ & None mentioned & None discussed & \\
\hline & \multicolumn{5}{|c|}{ Title of Reference: Enhanced In Situ Carbonation of Peridotite for Permanent $\mathrm{CO}_{2}$ Storage } \\
\hline \multirow[t]{2}{*}{ May (2005) } & Natural analogue & $\begin{array}{l}\text { Rhenish Massif, western } \\
\text { Germany and Belgium }\end{array}$ & $\begin{array}{l}\text { Siderite (most abundant } \\
\text { carbonate), dolomite-ankerite, } \\
\text { and calcite occur in altered rocks }\end{array}$ & Yes & PHREEQC \\
\hline & \multicolumn{5}{|c|}{$\begin{array}{l}\text { Title of Reference: Alteration of Wall Rocks by } \mathrm{CO}_{2} \text {-Rich Water Ascending in Fault Zones: Natural Analogues for Reactions } \\
\text { Induced by } \mathrm{CO}_{2} \text { Migrating along Faults in Siliciclastic Reservoir and Cap Rocks }\end{array}$} \\
\hline
\end{tabular}


Table 5.3. (contd)

\begin{tabular}{|c|c|c|c|c|c|}
\hline Reference & Study & Sites Studied & $\begin{array}{c}\text { Carbonate Minerals and } \\
\text { Reactions Physically } \\
\text { Observed }\end{array}$ & $\begin{array}{c}\text { Was } \\
\text { Geochemical } \\
\text { Reaction } \\
\text { Modeling } \\
\text { Used? }\end{array}$ & $\begin{array}{c}\text { Computer } \\
\text { Codes Used for } \\
\text { Geochemical } \\
\text { Reaction } \\
\text { Modeling }\end{array}$ \\
\hline \multirow{2}{*}{$\begin{array}{l}\text { Michael and } \\
\text { Buschkuehle (2006) }\end{array}$} & Natural analogue & $\begin{array}{l}\text { Sandstones in Halfway } \\
\text { Formation at West Stoddart, } \\
\text { British Columbia }\end{array}$ & $\begin{array}{l}\text { Presence of fine-grained } \\
\text { carbonate cements }\end{array}$ & Yes & GAMSPATH \\
\hline & \multicolumn{5}{|c|}{$\begin{array}{l}\text { Title of Reference: Acid-Gas Injection at West Stoddart, British Columbia: An Analogue for the Detailed Hydrogeological } \\
\text { Characterization of a } \mathrm{CO}_{2} \text { Sequestration Site }\end{array}$} \\
\hline \multirow[t]{2}{*}{ Moore et al. (2005) } & Natural analogue & $\begin{array}{l}\text { Springerville-St. Johns Field, } \\
\text { Arizona, and New Mexico, } \\
\text { United States }\end{array}$ & $\begin{array}{l}\text { Dissolution of authigenic } \\
\text { carbonate cement (dolomite) } \\
\text { and formation of dawsonite }\end{array}$ & Yes & $\begin{array}{l}\text { Geochemist's } \\
\text { Workbench }\end{array}$ \\
\hline & \multicolumn{5}{|c|}{$\begin{array}{l}\text { Title of Reference: Mineralogical and Geochemical Consequences of the Long-Term Presence of } \mathrm{CO}_{2} \text { in Natural Reservoirs: An } \\
\text { Example from the Springerville-St. Johns Field, Arizona, and New Mexico, USA }\end{array}$} \\
\hline \multirow{2}{*}{ Pauwels et al. (2007) } & Natural analogue & $\begin{array}{l}\text { Montmiral } \mathrm{CO}_{2} \text { accumulation, } \\
\text { southeastern France }\end{array}$ & $\begin{array}{l}\text { Dissolution of ferroan dolomite } \\
\text { and presence of minor ankerite }\end{array}$ & Yes & $\begin{array}{l}\text { SCALE; } \\
\text { PHREEQC }\end{array}$ \\
\hline & \multicolumn{5}{|c|}{$\begin{array}{l}\text { Title of Reference: Chemistry of Fluids from a Natural Analogue for a Geological } \mathrm{CO}_{2} \text { Storage Site (Montmiral, France): Lessons } \\
\text { for } \mathrm{CO}_{2} \text {-Water-Rock Interaction Assessment and Monitoring }\end{array}$} \\
\hline \multirow[t]{2}{*}{ Pearce et al. (1996) } & $\begin{array}{l}\text { Natural analogue } \\
\text { (review of information) } \\
\text { and experimental fluid- } \\
\text { rock experiments }\end{array}$ & $\begin{array}{l}\text { Analogue site - Tubb } \\
\text { Sandstones of Bravo Dome } \\
\mathrm{CO}_{2} \text {-field, New Mexico; } \\
\text { laboratory experiments with } \\
\text { anhydrite, Mercia Mudstone, } \\
\text { and Sherwood Sandstone from } \\
\text { United Kingdom }\end{array}$ & $\begin{array}{l}\text { Tubb Sandstones - dolomite as } \\
\text { lithoclasts and cement with } \\
\text { ferroan dolomite overgrowths, } \\
\text { and calcite in some samples as } \\
\text { intergranular cement } \\
\text { Anhydrite, mudstone, and } \\
\text { sandstone experiments - } \\
\text { dissolution of dolomite and } \\
\text { precipitation of secondary } \\
\text { calcite }\end{array}$ & None discussed & \\
\hline & \multicolumn{5}{|c|}{ Title of Reference: Natural Occurrences as Analogues for the Geological Disposal of Carbon Dioxide } \\
\hline
\end{tabular}


Table 5.3. (contd)

\begin{tabular}{|c|c|c|c|c|c|}
\hline Reference & Study & Sites Studied & $\begin{array}{c}\text { Carbonate Minerals and } \\
\text { Reactions Physically } \\
\text { Observed }\end{array}$ & $\begin{array}{l}\text { Was } \\
\text { Geochemical } \\
\text { Reaction } \\
\text { Modeling } \\
\text { Used? }\end{array}$ & $\begin{array}{l}\text { Computer } \\
\text { Codes Used for } \\
\text { Geochemical } \\
\text { Reaction } \\
\text { Modeling }\end{array}$ \\
\hline \multirow[t]{2}{*}{ Power et al. (2009) } & Natural analogue & $\begin{array}{l}\text { Hydromagnesite playas of Atlin, } \\
\text { British Columbia, Canada }\end{array}$ & $\begin{array}{l}\text { Precipitation of } \\
\text { hydromagnesite, nesquehonite, } \\
\text { and dypingite and also with } \\
\text { depth, ankerite, aragonite, and } \\
\text { magnesite }\end{array}$ & None discussed & \\
\hline & \multicolumn{5}{|c|}{$\begin{array}{l}\text { Title of Reference: The Hydromagnesite Playas of Atlin, British Columbia, Canada: A Biogeochemical Model for } \mathrm{CO}_{2} \\
\text { Sequestration }\end{array}$} \\
\hline \multirow{2}{*}{$\begin{array}{l}\text { Vosteen and May } \\
\text { (2007) (abstract only) }\end{array}$} & Natural analogue & $\begin{array}{l}\text { Bunter Sandstone and } \\
\text { Rotliegend sediments, Germany }\end{array}$ & $\begin{array}{l}\text { Dissolution of calcite and } \\
\text { precipitation of dolomite }\end{array}$ & Yes & Not identified \\
\hline & \multicolumn{5}{|c|}{ Title of Reference: Identification of Potential Geochemical Reactions in German Subsurface Storage Sites of $\mathrm{CO}_{2}$} \\
\hline \multirow[t]{2}{*}{ Watson et al. (2004) } & Natural analogue & $\begin{array}{l}\text { Siliciclastic reservoir-- } \\
\text { Ladbroke Grove and Katnook } \\
\text { Gas Fields within western } \\
\text { Otway Basin, southwestern } \\
\text { South Australia }\end{array}$ & $\begin{array}{l}\text { Ferroan carbonate is main } \\
\text { mineral product; } \\
\text { Pre- } \mathrm{CO}_{2} \text { calcite in Ladbroke } \\
\text { Grove dissolved but ankerite } \\
\text { and } \mathrm{Fe} \text { and } \mathrm{Mg} \text { carbonates } \\
\text { precipitated in microspar form }\end{array}$ & Yes & EQ3NR \\
\hline & \multicolumn{5}{|c|}{$\begin{array}{l}\text { Title of Reference: The Ladbroke Grove-Katnook Carbon Dioxide Natural Laboratory: A Recent } \mathrm{CO}_{2} \text { Accumulation in a Lithic } \\
\text { Sandstone Reservoir }\end{array}$} \\
\hline \multirow[t]{2}{*}{ Wilson et al. (2006) } & $\begin{array}{l}\text { Sample } \\
\text { characterization }\end{array}$ & $\begin{array}{l}\text { Chrysotile mine tailings at } \\
\text { Clinton Creek, Yukon Territory, } \\
\text { and Cassiar, British Columbia, } \\
\text { Canada }\end{array}$ & $\begin{array}{l}\text { Nesquehonite, dypingite, } \\
\text { hydromagnesite, and } \\
\text { sometimes lansfordite }\end{array}$ & None discussed & \\
\hline & \multicolumn{5}{|c|}{$\begin{array}{l}\text { Title of Reference: Verifying and Quantifying Carbon Fixation in Minerals from Serpentine-Rich Mine Tailings Using the Rietveld } \\
\text { Method with X-ray Powder Diffraction Data }\end{array}$} \\
\hline
\end{tabular}


Table 5.3. (contd)

\begin{tabular}{|c|c|c|c|c|c|}
\hline Reference & Study & Sites Studied & $\begin{array}{c}\text { Carbonate Minerals and } \\
\text { Reactions Physically } \\
\text { Observed }\end{array}$ & $\begin{array}{l}\text { Was } \\
\text { Geochemical } \\
\text { Reaction } \\
\text { Modeling } \\
\text { Used? }\end{array}$ & $\begin{array}{c}\text { Computer } \\
\text { Codes Used for } \\
\text { Geochemical } \\
\text { Reaction } \\
\text { Modeling }\end{array}$ \\
\hline \multirow[t]{2}{*}{ Wilson et al. (2009) } & $\begin{array}{l}\text { Sample } \\
\text { characterization }\end{array}$ & $\begin{array}{l}\text { Chrysotile mine tailings at } \\
\text { Clinton Creek, Yukon Territory, } \\
\text { and Cassiar, British Columbia, } \\
\text { Canada }\end{array}$ & $\begin{array}{l}\text { Nesquehonite, dypingite, } \\
\text { hydromagnesite, and } \\
\text { sometimes lansfordite }\end{array}$ & None discussed & \\
\hline & \multicolumn{5}{|c|}{$\begin{array}{l}\text { Title of Reference: Carbon Dioxide Fixation within Mine Wastes of Ultramafic-Hosted Ore Deposits: Examples from the Clinton } \\
\text { Creek and Cassiar Chrysotile Deposits, Canada }\end{array}$} \\
\hline \multirow[t]{2}{*}{ Worden (2006) } & Natural analogue & $\begin{array}{l}\text { Turbidite sandstones of the Lam } \\
\text { Formation in Yemen }\end{array}$ & $\begin{array}{l}\text { In sandstones, carbonate } \\
\text { cements include ferroan calcite, } \\
\text { dawsonite, ferroan dolomite, } \\
\text { and siderite }\end{array}$ & Yes & $\begin{array}{l}\text { Geochemist's } \\
\text { Workbench }\end{array}$ \\
\hline & \multicolumn{5}{|c|}{$\begin{array}{l}\text { Title of Reference: Dawsonite Cement in the Triassic Lam Formation, Shabwa Basin, Yemen: A Natural Analogue for a Potential } \\
\text { Mineral Product of Subsurface } \mathrm{CO}_{2} \text { Storage for Greenhouse Gas Reduction }\end{array}$} \\
\hline
\end{tabular}


Table 5.4. Published Studies of Laboratory Experiments Designed to Examine the Geochemical Reactions Associated with the Interaction of $\mathrm{CO}_{2}$-Containing Fluids with Characterized Rock, Single Mineral, or Cement Samples

\begin{tabular}{|c|c|c|c|c|c|}
\hline Reference & $\begin{array}{l}\text { Type of Laboratory } \\
\text { Study }\end{array}$ & Sites or Materials Studied & $\begin{array}{c}\text { Carbonate Minerals and } \\
\text { Reactions Physically } \\
\text { Observed }\end{array}$ & $\begin{array}{l}\text { Was } \\
\text { Geochemical } \\
\text { Reaction } \\
\text { Modeling } \\
\text { Used? }\end{array}$ & $\begin{array}{c}\text { Computer } \\
\text { Codes Used for } \\
\text { Geochemical } \\
\text { Reaction } \\
\text { Modeling }\end{array}$ \\
\hline \multirow[t]{2}{*}{$\begin{array}{l}\text { Alemu et al. (2009) } \\
\text { (abstract only) }\end{array}$} & Fluid-rock experiments & $\begin{array}{l}\text { Shale seal rock from } \\
\text { Adventdalen Group which is a } \\
\text { proposed } \mathrm{CO}_{2} \text { storage site in } \\
\text { Svalbard near Longyearbyen, } \\
\text { Norway }\end{array}$ & None discussed & Yes & PHREEQC \\
\hline & \multicolumn{5}{|c|}{$\begin{array}{l}\text { Title of Reference: Effect of } \mathrm{CO}_{2} \text { Interaction with Svalbard Shale: Implications to Caprock Integrity for Subsurface Sequestration } \\
\text { of } \mathrm{CO}_{2}\end{array}$} \\
\hline \multirow[t]{2}{*}{ Andreani et al. (2008) } & Fluid-rock experiments & $\begin{array}{l}\text { Claystone caprock (argillaceous } \\
\text { material) from the Upper } \\
\text { Toarcian formation of } \\
\text { Tournemire (France); bulk } \\
\text { material contains dominantly } \\
\text { clay minerals and lesser amounts } \\
\text { of calcite, quartz, siderite, and } \\
\text { pyrite; clay fraction contains } \\
\text { mostly kaolinite, micas } \\
\text { (muscovite), interstratified } \\
\text { illite/smectite, and chlorite }\end{array}$ & None identified & None discussed & \\
\hline & \multicolumn{5}{|c|}{ Title of Reference: Changes in Seal Capacity of Fractured Claystone Caprocks Induced by Dissolved and Gaseous $\mathrm{CO}_{2}$ Seepage } \\
\hline \multirow{2}{*}{ Andreani et al. (2009) } & Fluid-rock experiments & Sintered dunite (olivine) & $\begin{array}{l}\text { Formation of magnesite and } \\
\text { siderite }\end{array}$ & None discussed & \\
\hline & \multicolumn{5}{|c|}{$\begin{array}{l}\text { Title of Reference: Experimental Study of Carbon Sequestration Reactions Controlled by the Percolation of } \mathrm{CO}_{2} \text {-Rich Brine } \\
\text { through Peridotites }\end{array}$} \\
\hline
\end{tabular}


Table 5.4. (contd)

\begin{tabular}{|c|c|c|c|c|c|}
\hline Reference & $\begin{array}{l}\text { Type of Laboratory } \\
\text { Study }\end{array}$ & Sites or Materials Studied & $\begin{array}{c}\text { Carbonate Minerals and } \\
\text { Reactions Physically } \\
\text { Observed }\end{array}$ & $\begin{array}{l}\text { Was } \\
\text { Geochemical } \\
\text { Reaction } \\
\text { Modeling } \\
\text { Used? }\end{array}$ & $\begin{array}{c}\text { Computer } \\
\text { Codes Used for } \\
\text { Geochemical } \\
\text { Reaction } \\
\text { Modeling }\end{array}$ \\
\hline \multirow[t]{2}{*}{ Andreani et al. (2009) } & Fluid-rock experiments & $\begin{array}{l}\text { Dunite which contained } 97 \% \\
\text { olivine, } 2 \% \text { diopside, and 1\% } \\
\text { spinel (chromite) }\end{array}$ & $\begin{array}{l}\text { Two types of alteration } \\
\text { features - one with siderite and } \\
\text { other with Ca-enriched } \\
\text { magnesite in dissolution } \\
\text { surface on olivine }\end{array}$ & None discussed & \\
\hline & \multicolumn{5}{|c|}{$\begin{array}{l}\text { Title of Reference: Experimental Study of Carbon Sequestration Reactions Controlled by the Percolation of } \mathrm{CO}_{2} \text {-Rich Brine } \\
\text { through Peridotites }\end{array}$} \\
\hline \multirow{2}{*}{$\begin{array}{l}\text { Bachu and Bennion } \\
\text { (2009) }\end{array}$} & $\begin{array}{l}\text { Fluid-cement } \\
\text { experiments }\end{array}$ & Class G Portland cement & & None discussed & \\
\hline & \multicolumn{5}{|c|}{ Title of Reference: Experimental Assessment of Brine and/or $\mathrm{CO}_{2}$ Leakage through Well Cements at Reservoir Conditions } \\
\hline \multirow{2}{*}{$\begin{array}{l}\text { Barlet-Gouédard et al. } \\
\text { (2007) }\end{array}$} & $\begin{array}{l}\text { Fluid-cement } \\
\text { experiments }\end{array}$ & $\begin{array}{l}\text { Class G Portland cement and a } \\
\text { new } \mathrm{CO}_{2} \text {-resistant cement }\end{array}$ & $\begin{array}{l}\text { Aragonite, vaterite, and/or } \\
\text { calcite }\end{array}$ & None discussed & \\
\hline & \multicolumn{5}{|c|}{ Title of Reference: Well Technologies for $\mathrm{CO}_{2}$ Geological Storage: $\mathrm{CO}_{2}$-Resistant Cement } \\
\hline \multirow[t]{2}{*}{$\begin{array}{l}\text { Barlet-Gouédard et al. } \\
\text { (2009) }\end{array}$} & $\begin{array}{l}\text { Fluid-cement } \\
\text { experiments }\end{array}$ & $\begin{array}{l}\text { Portland cement and } \\
\text { Schlumberger (composition } \\
\text { proprietary) } \mathrm{CO}_{2} \text { resistant } \\
\text { cement }\end{array}$ & $\begin{array}{l}\text { Calcium carbonate (no } \\
\text { specifics) }\end{array}$ & None discussed & \\
\hline & \multicolumn{5}{|c|}{ 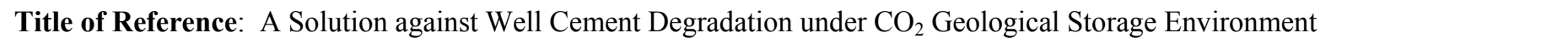 } \\
\hline \multirow[t]{2}{*}{ Bateman et al. (2005) } & Fluid-rock experiments & $\begin{array}{l}\text { Starting materials similar to } \\
\text { Saline Aquifer } \mathrm{CO}_{2} \text { Storage } \\
\text { (SACS) Project, Utsira } \\
\text { Formation, North Sea }\end{array}$ & $\begin{array}{l}\text { Dissolution of calcite and } \\
\text { dolomite with later } \\
\text { precipitation of dolomite }\end{array}$ & Yes & $\begin{array}{l}\text { PRECIP; } \\
\text { PHREEQC }\end{array}$ \\
\hline & \multicolumn{5}{|c|}{ Title of Reference: Large-Scale Column Experiment: Study of $\mathrm{CO}_{2}$, Porewater, Rock Reactions and Model Test Case } \\
\hline \multirow[t]{2}{*}{ Béarat et al. (2006) } & $\begin{array}{l}\text { Fluid-mineral } \\
\text { experiments }\end{array}$ & Olivine $\left[\left(\mathrm{Mg}_{0.915} \mathrm{Fe}_{0.085}\right)_{2} \mathrm{SiO}_{4}\right]$ & Magnesite & None discussed & \\
\hline & \multicolumn{5}{|c|}{ Title of Reference: Carbon Sequestration via Aqueous Olivine Mineral Carbonation: Role of Passivating Layer Formation } \\
\hline
\end{tabular}


Table 5.4. (contd)

\begin{tabular}{|c|c|c|c|c|c|}
\hline Reference & $\begin{array}{l}\text { Type of Laboratory } \\
\text { Study }\end{array}$ & Sites or Materials Studied & $\begin{array}{c}\text { Carbonate Minerals and } \\
\text { Reactions Physically } \\
\text { Observed }\end{array}$ & $\begin{array}{l}\text { Was } \\
\text { Geochemical } \\
\text { Reaction } \\
\text { Modeling } \\
\text { Used? }\end{array}$ & $\begin{array}{c}\text { Computer } \\
\text { Codes Used for } \\
\text { Geochemical } \\
\text { Reaction } \\
\text { Modeling }\end{array}$ \\
\hline \multirow[t]{2}{*}{ Bertier et al. (2006) } & Fluid-rock experiments & $\begin{array}{l}\text { Westphalian and Buntsandstein } \\
\text { Sandstones of the Campine } \\
\text { Basin, northeast Belgium }\end{array}$ & $\begin{array}{l}\text { Dissolution of ankerite/ } \\
\text { dolomite, and precipitation of } \\
\text { end-member carbonates } \\
\text { (siderite or pure calcite/ } \\
\text { aragonite) }\end{array}$ & None discussed & \\
\hline & \multicolumn{5}{|c|}{$\begin{array}{l}\text { Title of Reference: Experimental Identification of } \mathrm{CO}_{2} \text {-Water-Rock Interactions Caused by Sequestration of } \mathrm{CO}_{2} \text { in Westphalian } \\
\text { and Buntsandstein Sandstones of the Campine Basin (NE-Belgium) }\end{array}$} \\
\hline \multirow{2}{*}{$\begin{array}{l}\text { Bertier et al. (2007) } \\
\text { (abstract only) }\end{array}$} & Fluid-rock experiments & $\begin{array}{l}\text { Sandstone aquifers in the } \\
\text { Campine Basin, northeast } \\
\text { Belgium }\end{array}$ & None discussed & Yes & PHREEQE \\
\hline & \multicolumn{5}{|c|}{$\begin{array}{l}\text { Title of Reference: Evaluation of the } \mathrm{CO}_{2} \text {-Sequestration Capacity of Sandstone Aquifers in the Campine Basin (NE-Belgium) } \\
\text { Based on Autoclave Experiments and Numerical Modelling }\end{array}$} \\
\hline \multirow{2}{*}{$\begin{array}{l}\text { Brunet et al. (2009) } \\
\text { (abstract only) }\end{array}$} & $\begin{array}{l}\text { Fluid-cement } \\
\text { experiments }\end{array}$ & Portland cement & Calcite, aragonite, and vaterite & Yes & Not identified \\
\hline & \multicolumn{5}{|c|}{ Title of Reference: Calcium Carbonates Distribution in Experimentally Carbonated Portland Cement Cores } \\
\hline \multirow[t]{2}{*}{ Busch et al. (2008) } & Fluid-rock experiments & $\begin{array}{l}\text { Shale sample (Muderong Shale) } \\
\text { from Australia, and several } \\
\text { clays; shale contains siderite }\end{array}$ & $\begin{array}{l}\text { Siderite present in shale } \\
\text { sample; discussion of possible } \\
\text { formation of } \mathrm{Mg} \text {,Fe carbonates }\end{array}$ & None discussed & \\
\hline & \multicolumn{5}{|c|}{ Title of Reference: Carbon Dioxide Storage Potential of Shales } \\
\hline \multirow{2}{*}{$\begin{array}{l}\text { Centeno et al. (2005) } \\
\text { (abstract only) }\end{array}$} & $\begin{array}{l}\text { Fluid-cement } \\
\text { experiments }\end{array}$ & Oil well cement & None discussed & None discussed & \\
\hline & \multicolumn{5}{|c|}{ Title of Reference: Dissolution of Oil Well Cement in Presence of $\mathrm{CO}_{2} / \mathrm{H}_{2} \mathrm{~S}$ under HTHP } \\
\hline \multirow{2}{*}{$\begin{array}{l}\text { Centeno et al. (2007) } \\
\text { (abstract only) }\end{array}$} & $\begin{array}{l}\text { Fluid-cement } \\
\text { experiments }\end{array}$ & Class $\mathrm{G}$ and $\mathrm{H}$ cement & None discussed & None discussed & \\
\hline & \multicolumn{5}{|c|}{ Title of Reference: Durability and Degradation of Oil Well Cement Exposed to a Source of $\mathrm{H}_{2} \mathrm{~S}$ and $\mathrm{CO}_{2}$ Gases } \\
\hline
\end{tabular}


Table 5.4. (contd)

\begin{tabular}{|c|c|c|c|c|c|}
\hline Reference & $\begin{array}{l}\text { Type of Laboratory } \\
\text { Study }\end{array}$ & Sites or Materials Studied & $\begin{array}{c}\text { Carbonate Minerals and } \\
\text { Reactions Physically } \\
\text { Observed }\end{array}$ & $\begin{array}{l}\text { Was } \\
\text { Geochemical } \\
\text { Reaction } \\
\text { Modeling } \\
\text { Used? }\end{array}$ & $\begin{array}{c}\text { Computer } \\
\text { Codes Used for } \\
\text { Geochemical } \\
\text { Reaction } \\
\text { Modeling }\end{array}$ \\
\hline \multirow{2}{*}{$\begin{array}{l}\text { Centeno et al. (2009) } \\
\text { (abstract only) }\end{array}$} & $\begin{array}{l}\text { Fluid-cement } \\
\text { experiments }\end{array}$ & Class $\mathrm{G}$ and $\mathrm{H}$ cement & Vaterite, aragonite, and calcite & None discussed & \\
\hline & \multicolumn{5}{|c|}{ Title of Reference: SEM-EDS Applications in Mineralogical Phases Study of Oil Well Cements Attacked with $\mathrm{H}_{2} \mathrm{~S} / \mathrm{CO}_{2}$ Mixtures } \\
\hline \multirow[t]{2}{*}{$\begin{array}{l}\text { Charan and Begum } \\
\text { (2008) (abstract only) }\end{array}$} & Fluid-rock experiments & $\begin{array}{l}\text { Basalt samples from the } \\
\text { Cretaceous Deccan Continental } \\
\text { Flood Basalt Province, India }\end{array}$ & $\begin{array}{l}\text { Formation of carbonates; } \\
\text { specific mineral phases not } \\
\text { mentioned }\end{array}$ & None discussed & \\
\hline & \multicolumn{5}{|c|}{ Title of Reference: Geological Sequestration of Carbon-Dioxide in Deccan Continental Flood Basalt Province, India } \\
\hline \multirow[t]{2}{*}{$\begin{array}{l}\text { Charan et al. (2009) } \\
\text { (abstract only) }\end{array}$} & Fluid-rock experiments & $\begin{array}{l}\text { Basalt samples from the } \\
\text { Cretaceous Deccan Continental } \\
\text { Flood Basalt Province, India }\end{array}$ & $\begin{array}{l}\text { Formation of Fe-, C-, and } \mathrm{Mg}- \\
\text { containing carbonates that } \\
\text { matched well with ankerite, } \\
\text { calcite, and magnesite }\end{array}$ & None discussed & \\
\hline & \multicolumn{5}{|c|}{ Title of Reference: Mineral Trapping in Deccan Basalt Province India: Implications for Geological Sequestration of $\mathrm{CO}_{2}$} \\
\hline \multirow{2}{*}{$\begin{array}{l}\text { Choi et al. (2009) } \\
\text { (abstract only) }\end{array}$} & $\begin{array}{l}\text { Fluid-mineral } \\
\text { experiments }\end{array}$ & Anorthite & None discussed & None discussed & \\
\hline & \multicolumn{5}{|c|}{ Title of Reference: Dissolution of Anorthite under Supercritical $\mathrm{CO}_{2}$ - Water - Mineral Reaction System at a $\mathrm{CO}_{2}$ Sequestration Site } \\
\hline \multirow{2}{*}{ Daval et al. (2009) } & $\begin{array}{l}\text { Fluid-mineral } \\
\text { experiments }\end{array}$ & Wollastonite & Calcite & Yes & CHESS \\
\hline & \multicolumn{5}{|c|}{$\begin{array}{l}\text { Title of Reference: Carbonation of Ca-Bearing Silicates, the Case of Wollastonite: Experimental Investigations and Kinetic } \\
\text { Modeling }\end{array}$} \\
\hline \multirow[t]{2}{*}{ Dilmore et al. (2008a) } & $\begin{array}{l}\text { Fluid solubility } \\
\text { experiments }\end{array}$ & $\begin{array}{l}\text { Brine samples from Oriskany } \\
\text { Sandstone aquifer, Indiana } \\
\text { County, Pennsylvania, United } \\
\text { States }\end{array}$ & None discussed & Yes & $\begin{array}{l}\mathrm{CO}_{2} \text { gas } \\
\text { solubility model } \\
\text { of Duan and Sun } \\
(2003)\end{array}$ \\
\hline & \multicolumn{5}{|c|}{ Title of Reference: Sequestration of Dissolved $\mathrm{CO}_{2}$ in the Oriskany Formation } \\
\hline
\end{tabular}


Table 5.4. (contd)

\begin{tabular}{|c|c|c|c|c|c|}
\hline Reference & $\begin{array}{l}\text { Type of Laboratory } \\
\text { Study }\end{array}$ & Sites or Materials Studied & $\begin{array}{c}\text { Carbonate Minerals and } \\
\text { Reactions Physically } \\
\text { Observed }\end{array}$ & $\begin{array}{c}\text { Was } \\
\text { Geochemical } \\
\text { Reaction } \\
\text { Modeling } \\
\text { Used? }\end{array}$ & $\begin{array}{l}\text { Computer } \\
\text { Codes Used for } \\
\text { Geochemical } \\
\text { Reaction } \\
\text { Modeling }\end{array}$ \\
\hline \multirow{2}{*}{ Dilmore et al. (2008b) } & Fluid-rock experiments & Bauxite & $\begin{array}{l}\text { Calcite, magnesite, and } \\
\text { dawsonite }\end{array}$ & Yes & EQ3/EQ6 \\
\hline & \multicolumn{5}{|c|}{ Title of Reference: Sequestration of $\mathrm{CO}_{2}$ in Mixtures of Bauxite Residue and Saline Wastewater } \\
\hline \multirow{2}{*}{ Dufaud et al. (2009) } & $\begin{array}{l}\text { Fluid-mineral } \\
\text { experiments }\end{array}$ & $\begin{array}{l}\text { Olivines, orthopyroxenes, and } \\
\text { serpentines (chrysotile) }\end{array}$ & Magnesite & None discussed & \\
\hline & \multicolumn{5}{|c|}{ Title of Reference: Experimental Study of Mg-Rich Silicates Carbonation at 400 and $500{ }^{\circ} \mathrm{C}$ and $1 \mathrm{kbar}$} \\
\hline \multirow[t]{2}{*}{$\begin{array}{l}\text { Dupraz et al. (2007) } \\
\text { (abstract only) }\end{array}$} & $\begin{array}{l}\text { Fluid-biomineralization } \\
\text { experiments }\end{array}$ & $\begin{array}{l}\text { Carbonate mineral formation by } \\
\text { microorganisms }\end{array}$ & $\begin{array}{l}\text { Nucleation of carbonates on } \\
\text { bacteria followed by the } \\
\text { embedding of the cells within } \\
\text { the carbonate crystals }\end{array}$ & Yes & Chess 3.6 \\
\hline & \multicolumn{5}{|c|}{ Title of Reference: A Bacterial Model for Studying Interactions between Microorganisms and $\mathrm{CO}_{2}$ Injected in the Subsurface } \\
\hline \multirow{2}{*}{$\begin{array}{l}\text { Dupraz et al. (2008) } \\
\text { (abstract only) }\end{array}$} & $\begin{array}{l}\text { Fluid-biomineralization } \\
\text { experiments }\end{array}$ & $\begin{array}{l}\text { Mineral formation by } \\
\text { microorganisms }\end{array}$ & $\begin{array}{l}\text { Nucleation and growth studies; } \\
\text { specific phase not listed }\end{array}$ & Yes & Not identified \\
\hline & \multicolumn{5}{|c|}{ Title of Reference: Experimental Study of Biomineralization Processes Relevant to $\mathrm{CO}_{2}$ Geological Sequestration } \\
\hline \multirow{2}{*}{ Dupraz et al. (2009a) } & $\begin{array}{l}\text { Fluid-biomineralization } \\
\text { experiments }\end{array}$ & $\begin{array}{l}\text { Carbonate formation by } \\
\text { microorganisms }\end{array}$ & Aragonite, calcite, and vaterite & None discussed & \\
\hline & \multicolumn{5}{|c|}{ Title of Reference: Experimental Approach of $\mathrm{CO}_{2}$ Biomineralization in Deep Saline Aquifers } \\
\hline \multirow{2}{*}{ Dupraz et al. (2009b) } & $\begin{array}{l}\text { Fluid-biomineralization } \\
\text { experiments }\end{array}$ & $\begin{array}{l}\text { Carbonate formation by } \\
\text { microorganisms }\end{array}$ & $\begin{array}{l}\text { Precipitation of } \log \mathrm{Mg} \text { calcite } \\
\text { with small amounts of vaterite } \\
\text { and aragonite }\end{array}$ & Yes & CHESS \\
\hline & \multicolumn{5}{|c|}{$\begin{array}{l}\text { Title of Reference: Experimental and Numerical Modeling of Bacterially Induced pH Increase and Calcite Precipitation in Saline } \\
\text { Aquifers }\end{array}$} \\
\hline \multirow{2}{*}{$\begin{array}{l}\text { Fernandez-Bastero } \\
\text { et al. (2008) }\end{array}$} & $\begin{array}{l}\text { Fluid-mineral } \\
\text { experiments }\end{array}$ & Glauconite particles & None & None discussed & \\
\hline & \multicolumn{5}{|c|}{ Title of Reference: Kinetic and Structural Constraints during Glauconite Dissolution: Implications for Mineral Disposal of $\mathrm{CO}_{2}$} \\
\hline
\end{tabular}


Table 5.4. (contd)

\begin{tabular}{|c|c|c|c|c|c|}
\hline Reference & $\begin{array}{l}\text { Type of Laboratory } \\
\text { Study }\end{array}$ & Sites or Materials Studied & $\begin{array}{c}\text { Carbonate Minerals and } \\
\text { Reactions Physically } \\
\text { Observed }\end{array}$ & $\begin{array}{l}\text { Was } \\
\text { Geochemical } \\
\text { Reaction } \\
\text { Modeling } \\
\text { Used? }\end{array}$ & $\begin{array}{c}\text { Computer } \\
\text { Codes Used for } \\
\text { Geochemical } \\
\text { Reaction } \\
\text { Modeling }\end{array}$ \\
\hline \multirow{2}{*}{ Frost et al. (2008a) } & $\begin{array}{l}\text { Characterization } \\
\text { measurements }\end{array}$ & Coalingite and brugnatellite & Coalingite and brugnatellite & None discussed & \\
\hline & \multicolumn{5}{|c|}{$\begin{array}{l}\text { Title of Reference: Infrared and Infrared Emission Spectroscopic Study of Selected Magnesium Carbonate Minerals Containing } \\
\text { Ferric Iron - Implications for the Geosequestration of Greenhouse Gases }\end{array}$} \\
\hline \multirow{2}{*}{ Frost et al. (2008b) } & $\begin{array}{l}\text { Characterization } \\
\text { measurements }\end{array}$ & $\begin{array}{l}\text { Artinite, brugnatellite, and } \\
\text { dypingite }\end{array}$ & $\begin{array}{l}\text { Artinite, brugnatellite, and } \\
\text { dypingite }\end{array}$ & None discussed & \\
\hline & \multicolumn{5}{|c|}{$\begin{array}{l}\text { Title of Reference: Thermal Stability of Artinite, Dypingite and Brugnatellite - Implications for the Geosequestration of Green } \\
\text { House Gases }\end{array}$} \\
\hline \multirow[t]{2}{*}{$\begin{array}{l}\text { Garcia et al. (2008) } \\
\text { (abstract only) }\end{array}$} & $\begin{array}{l}\text { Fluid-mineral } \\
\text { experiments }\end{array}$ & $\begin{array}{l}\text { Pure minerals - olivine, } \\
\text { anorthite, and augite }\end{array}$ & $\begin{array}{l}\text { Olivine reacted to form } \\
\text { magnesite, anorthite to form } \\
\text { calcite, and augite to form } \\
\text { siderite and calcite }\end{array}$ & None discussed & \\
\hline & \multicolumn{5}{|c|}{ Title of Reference: Basic Minerals for Carbon Dioxide Sequestration: Potential and Quantification } \\
\hline \multirow{2}{*}{$\begin{array}{l}\text { Gudbrandsson et al. } \\
\text { (2009) } \\
\text { (abstract only) }\end{array}$} & $\begin{array}{l}\text { Fluid-rock dissolution } \\
\text { rate experiments }\end{array}$ & $\begin{array}{l}\text { Basalt from a dyke on Stapafell } \\
\text { Mountain on Reykjanes } \\
\text { peninsula in Iceland }\end{array}$ & None discussed & None discussed & \\
\hline & \multicolumn{5}{|c|}{ Title of Reference: Dissolution Rates of Crystalline Basalt as a Function of Temperature and Solution Composition } \\
\hline \multirow{2}{*}{$\begin{array}{l}\text { Guichet et al. (2005) } \\
\text { (abstract only) }\end{array}$} & Fluid-rock experiments & $\begin{array}{l}\text { Porous limestones from Lavous, } \\
\text { France }\end{array}$ & $\begin{array}{l}\text { Cores are pure essentially } \\
\text { calcite }\end{array}$ & Yes & DIAPHORE \\
\hline & \multicolumn{5}{|c|}{ Title of Reference: Reactive Transport Experiments and Modelling of $\mathrm{CO}_{2}$ Sequestration in Deep Aquifers } \\
\hline \multirow{2}{*}{ Gunter et al. (1997) } & Fluid-rock experiments & $\begin{array}{l}\text { Glauconitic sandstone from } \\
\text { Alberta Sedimentary Basin, } \\
\text { Canada }\end{array}$ & No carbonate minerals detected & Yes & PATHARC.94 \\
\hline & \multicolumn{5}{|c|}{$\begin{array}{l}\text { Title of Reference: Aquifer Disposal of } \mathrm{CO}_{2} \text {-Rich Greenhouse Gases: Extension of the Time Scale of Experiment for } \\
\mathrm{CO}_{2} \text {-Sequestering Reactions by Geochemical Modelling }\end{array}$} \\
\hline
\end{tabular}


Table 5.4. (contd)

\begin{tabular}{|c|c|c|c|c|c|}
\hline Reference & $\begin{array}{l}\text { Type of Laboratory } \\
\text { Study }\end{array}$ & Sites or Materials Studied & $\begin{array}{c}\text { Carbonate Minerals and } \\
\text { Reactions Physically } \\
\text { Observed }\end{array}$ & $\begin{array}{l}\text { Was } \\
\text { Geochemical } \\
\text { Reaction } \\
\text { Modeling } \\
\text { Used? }\end{array}$ & $\begin{array}{l}\text { Computer } \\
\text { Codes Used for } \\
\text { Geochemical } \\
\text { Reaction } \\
\text { Modeling }\end{array}$ \\
\hline \multirow{2}{*}{$\begin{array}{l}\text { Gysi and Stefánsson } \\
\text { (2009) } \\
\text { (abstract only) }\end{array}$} & Fluid-rock experiments & $\begin{array}{l}\text { Basalt related to pilot field study } \\
\text { in southwestern Iceland }\end{array}$ & Fe-Mg-Ca-Mn carbonates & Yes & Not identified \\
\hline & \multicolumn{5}{|c|}{ Title of Reference: $\mathrm{CO}_{2}$-Water-Basalt Interaction: Geochemical Modelling and Experiments } \\
\hline \multirow{2}{*}{ Hänchen et al. (2008) } & $\begin{array}{l}\text { Mineral precipitation } \\
\text { experiments }\end{array}$ & $\begin{array}{l}\text { Mixing } \mathrm{Na}_{2} \mathrm{CO}_{3} \text { and } \mathrm{MgCl}_{2} \\
\text { solutions with } \mathrm{CO}_{2} \text { atmosphere }\end{array}$ & $\begin{array}{l}\text { Nesquehonite, hydromagnesite, } \\
\text { magnesite }\end{array}$ & Yes & EQ3/EQ6 \\
\hline & \multicolumn{5}{|c|}{ Title of Reference: Precipitation in the Mg-Carbonate System - Effects of Temperature and $\mathrm{CO}_{2}$ Pressure } \\
\hline \multirow{2}{*}{$\begin{array}{l}\text { Hangx and Spiers } \\
(2009)\end{array}$} & $\begin{array}{l}\text { Fluid-mineral } \\
\text { experiments }\end{array}$ & Anorthite and albite & $\begin{array}{l}\text { Precipitations of hydrotalcite } \\
\text { and some aragonite }\end{array}$ & None discussed & \\
\hline & \multicolumn{5}{|c|}{ Title of Reference: Reaction of Plagioclase Feldspars with $\mathrm{CO}_{2}$ under Hydrothermal Conditions } \\
\hline \multirow{2}{*}{$\begin{array}{l}\text { Iglesias et al. (2008) } \\
\text { (abstract only) }\end{array}$} & Fluid-rock experiments & $\begin{array}{l}\text { Subarkose sandstone from the } \\
\text { Rio Bonito Formation aquifer in } \\
\text { the Paraná Basin, Brazil }\end{array}$ & $\begin{array}{l}\text { Precipitation of calcium and } \\
\text { iron carbonates (mineral forms } \\
\text { not specified in abstract) }\end{array}$ & Yes & PHREEQC \\
\hline & \multicolumn{5}{|c|}{$\begin{array}{l}\text { Title of Reference: Experimental and Modeling Studies of } \mathrm{CO}_{2} \text {-Water-Rock Interactions in the Rio Bonito Saline Aquifer, Paraná } \\
\text { Basin, Brazil }\end{array}$} \\
\hline \multirow[t]{2}{*}{ Izgec et al. (2008a) } & Fluid-rock experiments & $\begin{array}{l}\text { Cores from carbonate } \\
\text { formations from Midyat } \\
\text { formation, south east Turkey, } \\
\text { and from a quarry, } \\
\text { St. Maximim, France }\end{array}$ & Precipitation of calcite & Yes & $\begin{array}{l}\text { STARS, see } \\
\text { Izgec et al. } \\
\text { (2008b) in } \\
\text { Table } 2\end{array}$ \\
\hline & \multicolumn{5}{|c|}{ Title of Reference: $\mathrm{CO}_{2}$ Injection into Saline Carbonate Aquifer Formations I: Laboratory Investigation } \\
\hline \multirow{2}{*}{ Jacquemet et al. (2008) } & $\begin{array}{l}\text { Fluid-cement } \\
\text { experiments }\end{array}$ & $\begin{array}{l}\text { Well cement (crushed) and well } \\
\text { casing steel }\end{array}$ & $\begin{array}{l}\text { Precipitation of calcite or } \\
\text { aragonite in crushed cement }\end{array}$ & None discussed & \\
\hline & \multicolumn{5}{|c|}{ Title of Reference: Mineralogical Changes of a Well Cement in Various $\mathrm{H}_{2} \mathrm{~S}_{-}-\mathrm{CO}_{2}(-\mathrm{Brine})$ Fluids at High Pressure and Temperature } \\
\hline \multirow{2}{*}{ Jarvis et al. (2009) } & $\begin{array}{l}\text { Fluid-mineral } \\
\text { experiments }\end{array}$ & $\begin{array}{l}\text { Olivine }\left(\mathrm{Mg}_{2} \mathrm{SiO}_{4}\right) \text { containing } \\
8 \% \mathrm{Fe}\end{array}$ & Precipitation of magnesite & Yes & $\mathrm{EQ} 3 / 6$ \\
\hline & \multicolumn{5}{|c|}{ Title of Reference: Reaction Mechanisms for Enhancing Mineral Sequestration of $\mathrm{CO}_{2}$} \\
\hline
\end{tabular}


Table 5.4. (contd)

\begin{tabular}{|c|c|c|c|c|c|}
\hline Reference & $\begin{array}{l}\text { Type of Laboratory } \\
\text { Study }\end{array}$ & Sites or Materials Studied & $\begin{array}{c}\text { Carbonate Minerals and } \\
\text { Reactions Physically } \\
\text { Observed }\end{array}$ & $\begin{array}{c}\text { Was } \\
\text { Geochemical } \\
\text { Reaction } \\
\text { Modeling } \\
\text { Used? } \\
\end{array}$ & $\begin{array}{c}\text { Computer } \\
\text { Codes Used for } \\
\text { Geochemical } \\
\text { Reaction } \\
\text { Modeling } \\
\end{array}$ \\
\hline \multirow[t]{2}{*}{ Kaszuba et al. (2003) } & Fluid-rock experiment & $\begin{array}{l}\text { Simulated arkose to represent } \\
\text { aquifer and Maplewood Shale } \\
\text { (argillaceous share) from } \\
\text { Monroe County, New York, } \\
\text { United States as the aquitard }\end{array}$ & $\begin{array}{l}\text { Precipitated magnesite } \\
\text { (desiccation of brine - by } \\
\text { extraction of water from brine } \\
\text { into injected } \mathrm{scCO}_{2} \text { ) }\end{array}$ & None discussed & \\
\hline & \multicolumn{5}{|c|}{$\begin{array}{l}\text { Title of Reference: Carbon Dioxide Reaction Processes in a Model Brine Aquifer at } 200^{\circ} \mathrm{C} \text { and } 200 \text { Bars: Implications for Geologic } \\
\text { Sequestration of Carbon }\end{array}$} \\
\hline \multirow[t]{2}{*}{ Kaszuba et al. (2005) } & Fluid-rock experiment & $\begin{array}{l}\text { Simulated arkose to represent } \\
\text { aquifer and Maplewood Shale } \\
\text { (argillaceous share) from } \\
\text { Monroe County, New York, } \\
\text { United States as the aquitard }\end{array}$ & $\begin{array}{l}\text { Precipitation corroded } \\
\text { magnesite followed by siderite }\end{array}$ & Yes & No details \\
\hline & \multicolumn{5}{|c|}{$\begin{array}{l}\text { Title of Reference: Experimental Evaluation of Mixed Fluid Reactions between Supercritical Carbon Dioxide and NaCl Brine: } \\
\text { Relevance to the Integrity of a Geologic Carbon Repository }\end{array}$} \\
\hline \multirow[t]{2}{*}{ Ketzer et al. (2009) } & Fluid-rock experiments & $\begin{array}{l}\text { Sandstones from the saline } \\
\text { reservoir of the Rio Bonito } \\
\text { Formation, Paraná Basin in } \\
\text { southern Brazil }\end{array}$ & $\begin{array}{l}\text { Samples contained calcite or } \\
\text { dolomite; identified } \\
\text { precipitation of pure end- } \\
\text { member calcite and ferroan } \\
\text { calcite }\end{array}$ & Yes & PHREEQC \\
\hline & \multicolumn{5}{|c|}{$\begin{array}{l}\text { Title of Reference: Water-Rock- } \mathrm{CO}_{2} \text { Interactions in Saline Aquifers Aimed for Carbon Dioxide Storage: Experimental and } \\
\text { Numerical Modeling Studies of the Rio Bonito Formation (Permian), Southern Brazil }\end{array}$} \\
\hline \multirow{2}{*}{$\begin{array}{l}\text { Kunieda et al. (2008) } \\
\text { (abstract only) }\end{array}$} & $\begin{array}{l}\text { Fluid-cement } \\
\text { experiments }\end{array}$ & $\begin{array}{l}\text { Cylinder of Berea sandstone } \\
\text { with a center hole filled with oil } \\
\text { well cement }\end{array}$ & $\begin{array}{l}\text { Precipitation of aragonite and } \\
\text { calcite }\end{array}$ & None discussed & \\
\hline & \multicolumn{5}{|c|}{$\begin{array}{l}\text { Title of Reference: Experimental Research of Casing Cement Alteration by Interaction with Supercritical } \mathrm{CO}_{2} \text { for Geological } \\
\text { Sequestration }\end{array}$} \\
\hline
\end{tabular}


Table 5.4. (contd)

\begin{tabular}{|c|c|c|c|c|c|}
\hline Reference & $\begin{array}{l}\text { Type of Laboratory } \\
\text { Study }\end{array}$ & Sites or Materials Studied & $\begin{array}{c}\text { Carbonate Minerals and } \\
\text { Reactions Physically } \\
\text { Observed }\end{array}$ & $\begin{array}{l}\text { Was } \\
\text { Geochemical } \\
\text { Reaction } \\
\text { Modeling } \\
\text { Used? }\end{array}$ & $\begin{array}{l}\text { Computer } \\
\text { Codes Used for } \\
\text { Geochemical } \\
\text { Reaction } \\
\text { Modeling }\end{array}$ \\
\hline \multirow{2}{*}{$\begin{array}{l}\text { Kuroda et al. (2008) } \\
\text { (abstract only) }\end{array}$} & $\begin{array}{l}\text { Fluid-mineral } \\
\text { experiments }\end{array}$ & Anorthite & Precipitation of calcite & Yes & No details \\
\hline & \multicolumn{5}{|c|}{$\begin{array}{l}\text { Title of Reference: Experimental Research of Plagioclase-Gas-Water Interaction at Hydrothermal Conditions Caused by } \mathrm{CO}_{2} \\
\text { Sequestration }\end{array}$} \\
\hline \multirow[t]{2}{*}{ Kutchko et al. (2007) } & $\begin{array}{l}\text { Fluid-cement } \\
\text { experiments }\end{array}$ & Class H Portland cement & Calcite & None discussed & \\
\hline & \multicolumn{5}{|c|}{ Title of Reference: Degradation of Well Cement by $\mathrm{CO}_{2}$ under Geologic Sequestration Conditions } \\
\hline \multirow[t]{2}{*}{ Kutchko et al. (2008) } & $\begin{array}{l}\text { Fluid-cement } \\
\text { experiments }\end{array}$ & Class H Portland cement & $\begin{array}{l}\text { Calcium carbonate (specific } \\
\text { phases not identified) [see } \\
\text { Kutchko et al. (2007)] }\end{array}$ & None discussed & \\
\hline & \multicolumn{5}{|c|}{ Title of Reference: Rate of $\mathrm{CO}_{2}$ Attack on Hydrated Class $\mathrm{H}$ Well Cement under Geologic Sequestration Conditions } \\
\hline \multirow{2}{*}{ Kutchko et al. (2009) } & $\begin{array}{l}\text { Fluid-cement } \\
\text { experiments }\end{array}$ & $\begin{array}{l}\text { Class H Portland cement } \\
\text { amended with Type F flyash }\end{array}$ & Calcite, aragonite, and vaterite & None discussed & \\
\hline & \multicolumn{5}{|c|}{$\begin{array}{l}\text { Title of Reference: } \mathrm{CO}_{2} \text { Reaction with Hydrated Class H Well Cement under Geologic Sequestration Conditions: Effects of Flyash } \\
\text { Admixtures }\end{array}$} \\
\hline \multirow{2}{*}{ Lin et al. (2008) } & $\begin{array}{l}\text { Fluid-rock and fluid- } \\
\text { mineral experiments }\end{array}$ & $\begin{array}{l}\text { Quartz, biotite, and iidate } \\
\text { granite }\end{array}$ & Precipitation of calcite & None discussed & \\
\hline & \multicolumn{5}{|c|}{$\begin{array}{l}\text { Title of Reference: Experimental Evaluation of Interactions in Supercritical } \mathrm{CO}_{2} / \text { Water/Rock Minerals System under Geologic } \mathrm{CO}_{2} \\
\text { Sequestration Conditions }\end{array}$} \\
\hline \multirow[t]{2}{*}{ Liu et al. (2003) } & Fluid-rock experiments & $\begin{array}{l}\text { Iidate Granite from Fukushima } \\
\text { Prefecture, Japan; Kimachi } \\
\text { Sandstone from Shimane } \\
\text { Prefecture, Japan }\end{array}$ & None discussed & None discussed & \\
\hline & \multicolumn{5}{|c|}{ Title of Reference: $\mathrm{CO}_{2}$ Injection to Granite and Sandstone in Experimental Rock/Hot Water Systems } \\
\hline
\end{tabular}


Table 5.4. (contd)

\begin{tabular}{|c|c|c|c|c|c|}
\hline Reference & $\begin{array}{l}\text { Type of Laboratory } \\
\text { Study }\end{array}$ & Sites or Materials Studied & $\begin{array}{c}\text { Carbonate Minerals and } \\
\text { Reactions Physically } \\
\text { Observed }\end{array}$ & $\begin{array}{c}\text { Was } \\
\text { Geochemical } \\
\text { Reaction } \\
\text { Modeling } \\
\text { Used? }\end{array}$ & $\begin{array}{c}\text { Computer } \\
\text { Codes Used for } \\
\text { Geochemical } \\
\text { Reaction } \\
\text { Modeling }\end{array}$ \\
\hline \multirow[t]{2}{*}{$\begin{array}{l}\text { Luquot and Gouze } \\
(2009)\end{array}$} & Fluid-rock experiments & $\begin{array}{l}\text { Oolitic limestone from the } \\
\text { Mondeville formation in the } \\
\text { Paris Basin in France }\end{array}$ & $\begin{array}{l}\text { Initial samples contained } \\
\mathrm{Mg} \text {-calcite }\left(\mathrm{Ca}_{0.99} \mathrm{Mg}_{0.01} \mathrm{CO}_{3}\right) \\
\text { precipitation occurred of } \\
\text { Mg-calcite of composition } \\
\mathrm{Ca}_{0.92} \mathrm{Mg}_{0.08} \mathrm{CO}_{3} \text { at the oolith } \\
\text { surfaces }\end{array}$ & Yes & CHESS \\
\hline & \multicolumn{5}{|c|}{$\begin{array}{l}\text { Title of Reference: Experimental Determination of Porosity and Permeability Changes Induced by Injection of } \mathrm{CO}_{2} \text { into Carbonate } \\
\text { Rocks }\end{array}$} \\
\hline \multirow{2}{*}{$\begin{array}{l}\text { Martinez et al. (2007) } \\
\text { (abstract only) }\end{array}$} & $\begin{array}{l}\text { Fluid-biomineral } \\
\text { experiments }\end{array}$ & $\begin{array}{l}\text { Carbonate mineral formation by } \\
\text { hotosynthetic cyanobacteria }\end{array}$ & None discussed & None discussed & \\
\hline & \multicolumn{5}{|c|}{ Title of Reference: Characterization of Carbonate Mineral Formation by Cyanobacteria and the Implications in $\mathrm{CO}_{2}$ Sequestration } \\
\hline \multirow[t]{2}{*}{ McGrail et al. (2006) } & Fluid-rock experiments & Basalt & $\begin{array}{l}\text { Precipitation of calcite that } \\
\text { underwent phase transition to } \\
\text { ankerite with extended time }\end{array}$ & Yes & EQ3NR \\
\hline & \multicolumn{5}{|c|}{ Title of Reference: Potential for Carbon Dioxide Sequestration in Flood Basalts } \\
\hline \multirow[t]{2}{*}{ McKelvy et al. (2004) } & $\begin{array}{l}\text { Characterization } \\
\text { studies and fluid-rock } \\
\text { experiments }\end{array}$ & $\begin{array}{l}\text { Lizardite as the model } \\
\text { serpentine material }\end{array}$ & Magnesite & None discussed & \\
\hline & \multicolumn{5}{|c|}{ Title of Reference: Exploration of the Role of Heat Activation in Enhancing Serpentine Carbon Sequestration Reactions } \\
\hline \multirow[t]{2}{*}{$\begin{array}{l}\text { Mitchell et al. (2008) } \\
\text { (abstract only) }\end{array}$} & $\begin{array}{l}\text { Biomineralization and } \\
\text { biofilm barrier } \\
\text { experiments }\end{array}$ & Mineral formation by biofilms & $\begin{array}{l}\text { Calcium carbonate; specific } \\
\text { form not identified }\end{array}$ & None discussed & \\
\hline & \multicolumn{5}{|c|}{ Title of Reference: Microbially Enhanced Carbonate Mineralization and the Geologic Containment of $\mathrm{CO}_{2}$} \\
\hline \multirow[t]{2}{*}{ Mitchell et al. (2009) } & $\begin{array}{l}\text { Biofilm formation } \\
\text { experiments }\end{array}$ & Berea sandstone & None discussed & None discussed & \\
\hline & \multicolumn{5}{|c|}{ Title of Reference: Biofilm Enhanced Geologic Sequestration of Supercritical $\mathrm{CO}_{2}$} \\
\hline
\end{tabular}


Table 5.4. (contd)

\begin{tabular}{|c|c|c|c|c|c|}
\hline Reference & $\begin{array}{c}\text { Type of Laboratory } \\
\text { Study } \\
\end{array}$ & Sites or Materials Studied & $\begin{array}{c}\text { Carbonate Minerals and } \\
\text { Reactions Physically } \\
\text { Observed }\end{array}$ & $\begin{array}{c}\text { Was } \\
\text { Geochemical } \\
\text { Reaction } \\
\text { Modeling } \\
\text { Used? } \\
\end{array}$ & $\begin{array}{c}\text { Computer } \\
\text { Codes Used for } \\
\text { Geochemical } \\
\text { Reaction } \\
\text { Modeling } \\
\end{array}$ \\
\hline \multirow[t]{2}{*}{ Mito et al. (2008) } & $\begin{array}{l}\text { Fluid-rock experiments } \\
\text { and field sample study }\end{array}$ & $\begin{array}{l}\text { Sandstone bed (Minami- } \\
\text { Nagaoka gas field) of the } \\
\text { Haizume Formation at Nagaoka, } \\
\text { Japan }\end{array}$ & $\begin{array}{l}\text { Calcite observed in original } \\
\text { reservoir rock; characterization } \\
\text { of specific carbonate mineral } \\
\text { products were not identified }\end{array}$ & Yes & $\begin{array}{l}\text { PHREEQC, } \\
\text { ChemTOUGH }\end{array}$ \\
\hline & \multicolumn{5}{|c|}{ Title of Reference: Case Study of Geochemical Reactions at the Nagaoka $\mathrm{CO}_{2}$ Injection Site, Japan } \\
\hline \multirow[t]{2}{*}{ Noiriel et al. (2009) } & Fluid-rock experiments & $\begin{array}{l}\text { Limestone from a borehole } \\
\text { drilled in the Campos Basin in } \\
\text { Mallorca, Balearic Islands, } \\
\text { Spain }\end{array}$ & $\begin{array}{l}\text { Limestone contains only } \\
\text { calcite, which occurred in two } \\
\text { forms - micrite grains and } \\
\text { sparite crystals which have } \\
\text { different trace element } \\
\text { signatures }\end{array}$ & None discussed & \\
\hline & \multicolumn{5}{|c|}{ Title of Reference: Changes in Reactive Surface Area during Limestone Dissolution: An Experimental and Modelling Study } \\
\hline \multirow{2}{*}{ Palandri et al. (2005) } & $\begin{array}{l}\text { Fluid-mineral } \\
\text { experiments }\end{array}$ & $\begin{array}{l}\text { Hematite (reduction of } \mathrm{Fe}^{\mathrm{III}} \text { to } \\
\mathrm{Fe}^{\mathrm{II}} \text { ) }\end{array}$ & $\begin{array}{l}\text { Precipitation of siderite and } \\
\text { dawsonite }\end{array}$ & Yes & CHILLER \\
\hline & \multicolumn{5}{|c|}{ Title of Reference: Ferric Iron in Sediments as a Novel $\mathrm{CO}_{2}$ Mineral Trap: $\mathrm{CO}_{2}-\mathrm{SO}_{2}$ Reaction with Hematite } \\
\hline \multirow[t]{2}{*}{ Park and Fan (2004) } & Fluid-rock experiments & Serpentine & $\begin{array}{l}\text { Precipitation of magnesium } \\
\text { carbonate }\end{array}$ & None discussed & \\
\hline & \multicolumn{5}{|c|}{ Title of Reference: $\mathrm{CO}_{2}$ Mineral Sequestration: Physically Activated Dissolution of Serpentine and pH Swing Process } \\
\hline \multirow[t]{2}{*}{ Prasad et al. (2009) } & Fluid-rock experiments & $\begin{array}{l}\text { Picritic basalts from Deccan } \\
\text { basalts in India }\end{array}$ & Precipitation of ankerite & None discussed & \\
\hline & \multicolumn{5}{|c|}{ Title of Reference: Geological Sequestration of Carbon Dioxide in Deccan Basalts: Preliminary Laboratory Study } \\
\hline \multirow[t]{2}{*}{ Regnault et al. (2009) } & $\begin{array}{l}\text { Fluid-mineral(cement) } \\
\text { experiments }\end{array}$ & $\begin{array}{l}\text { Portlandite because it is a main } \\
\text { component of borehole cement }\end{array}$ & Calcite & Yes & Not identified \\
\hline & \multicolumn{5}{|c|}{ Title of Reference: Experimental Measurement of Portlandite Carbonation Kinetics with Supercritical $\mathrm{CO}_{2}$} \\
\hline
\end{tabular}


Table 5.4. (contd)

\begin{tabular}{|c|c|c|c|c|c|}
\hline Reference & $\begin{array}{l}\text { Type of Laboratory } \\
\text { Study }\end{array}$ & Sites or Materials Studied & $\begin{array}{c}\text { Carbonate Minerals and } \\
\text { Reactions Physically } \\
\text { Observed }\end{array}$ & $\begin{array}{c}\text { Was } \\
\text { Geochemical } \\
\text { Reaction } \\
\text { Modeling } \\
\text { Used? }\end{array}$ & $\begin{array}{c}\text { Computer } \\
\text { Codes Used for } \\
\text { Geochemical } \\
\text { Reaction } \\
\text { Modeling }\end{array}$ \\
\hline \multirow[t]{2}{*}{ Rimmelé et al. (2008) } & $\begin{array}{l}\text { Fluid-cement } \\
\text { experiments }\end{array}$ & Class G Portland cement & Calcite and aragonite & None discussed & \\
\hline & \multicolumn{5}{|c|}{ Title of Reference: Heterogeneous Porosity Distribution in Portland Cement Exposed to $\mathrm{CO}_{2}$-Rich Fluids } \\
\hline \multirow{2}{*}{$\begin{array}{l}\text { Rosenbauer et al. } \\
(2005)\end{array}$} & Fluid-rock experiments & $\begin{array}{l}\text { Limestone; plagioclase-rich } \\
\text { arkosic sandstone }\end{array}$ & $\begin{array}{l}\text { Dissolution of calcite and } \\
\text { dolomitization of limestone }\end{array}$ & Yes & $\begin{array}{l}\text { SOLMINEQ99, } \\
\text { CHILLER, } \\
\text { SOLVEQ }\end{array}$ \\
\hline & \multicolumn{5}{|c|}{$\begin{array}{l}\text { Title of Reference: Experimental Investigation of } \mathrm{CO}_{2} \text {-Brine-Rock Interactions at Elevated Temperature and Pressure: Implication } \\
\text { for } \mathrm{CO}_{2} \text { Sequestration in Deep-Saline Aquifers }\end{array}$} \\
\hline \multirow{2}{*}{$\begin{array}{l}\text { Rosenbauer and } \\
\text { Bischoff (2009) } \\
\text { (abstract only) }\end{array}$} & Fluid-rock experiments & Basalt & None discussed & Yes & Not identified \\
\hline & \multicolumn{5}{|c|}{ Title of Reference: $\mathrm{CO}_{2}$ Sequestration by Basalt: Experimental studies and Geochemical Modeling } \\
\hline \multirow[t]{2}{*}{ Sass et al. (2001) } & $\begin{array}{l}\text { Fluid-rock and fluid- } \\
\text { mineral experiments }\end{array}$ & $\begin{array}{l}\text { Mt. Simon sandstone (potential } \\
\text { reservoir), Eau Claire shale, and } \\
\text { Rome dolomite (caprock for } \\
\text { Mt. Simon), Ohio; pure minerals } \\
\text { (high Ca plagioclase, anorthite, } \\
\text { glauconite, kaolinite, } \\
\text { montmorillonite) }\end{array}$ & $\begin{array}{l}\text { No carbonate precipitation } \\
\text { observed }\end{array}$ & Yes & $\begin{array}{l}\text { PHRQPITZ, } \\
\text { PHREEQC }\end{array}$ \\
\hline & \multicolumn{5}{|c|}{ Title of Reference: Geochemical Evaluation of Carbon Dioxide Sequestration in Saline Formations - Final Technical Report } \\
\hline \multirow[t]{2}{*}{ Sass et al. (2002) } & $\begin{array}{l}\text { Fluid-mineral } \\
\text { experiments }\end{array}$ & $\begin{array}{l}\text { Pure minerals (glauconite and } \\
\text { anorthite) }\end{array}$ & $\begin{array}{l}\text { No carbonate precipitation } \\
\text { observed }\end{array}$ & Yes & Not identified \\
\hline & \multicolumn{5}{|c|}{ Title of Reference: Interaction of Rock Minerals with Carbon Dioxide and Brine: A Hydrothermal Investigation } \\
\hline \multirow[t]{2}{*}{$\begin{array}{l}\text { Schaef and McGrail } \\
\text { (2009) }\end{array}$} & Fluid-rock experiments & $\begin{array}{l}\text { Basalt samples from Grand } \\
\text { Ronde Basalt Formation } \\
\text { collected near Vantage, } \\
\text { Washington }\end{array}$ & None discussed & Yes & EQ3NR \\
\hline & \multicolumn{5}{|c|}{$\begin{array}{l}\text { Title of Reference: Dissolution of Columbia River Basalt Under Mildly Acidic Conditions as a Function of Temperature: } \\
\text { Experimental Results Relevant to the Geological Sequestration of Carbon Dioxide }\end{array}$} \\
\hline
\end{tabular}


Table 5.4. (contd)

\begin{tabular}{|c|c|c|c|c|c|}
\hline Reference & $\begin{array}{l}\text { Type of Laboratory } \\
\text { Study }\end{array}$ & Sites or Materials Studied & $\begin{array}{c}\text { Carbonate Minerals and } \\
\text { Reactions Physically } \\
\text { Observed }\end{array}$ & $\begin{array}{c}\text { Was } \\
\text { Geochemical } \\
\text { Reaction } \\
\text { Modeling } \\
\text { Used? }\end{array}$ & $\begin{array}{l}\text { Computer } \\
\text { Codes Used for } \\
\text { Geochemical } \\
\text { Reaction } \\
\text { Modeling }\end{array}$ \\
\hline \multirow[t]{2}{*}{ Schaef et al. (2010) } & Fluid-rock experiments & $\begin{array}{l}\text { Basalt samples from Columbia } \\
\text { River basalt from Grande Ronde } \\
\text { Formation in Washington; } \\
\text { Newark Basin in South } \\
\text { Carolina; Deccan basalt from } \\
\text { Khandala Formation in India; } \\
\text { and Karoo Formation in South } \\
\text { Africa }\end{array}$ & $\begin{array}{l}\text { Precipitation of Ca carbonates } \\
\text { (including calcite) also } \\
\text { containing } \mathrm{Mg}, \mathrm{Fe} \text {, and } \mathrm{Mn} \text {; } \\
\text { and precipitation of } \\
\text { rhodochrosite }\end{array}$ & Yes & EQ3/EQ6 \\
\hline & \multicolumn{5}{|c|}{ Title of Reference: Carbonate Mineralization of Volcanic Province Basalts } \\
\hline \multirow[t]{2}{*}{ Scherer et al. (2005) } & $\begin{array}{l}\text { Fluid-rock-cement } \\
\text { experiments }\end{array}$ & $\begin{array}{l}\text { Cylinders of Chass H Portland } \\
\text { cement inside holes along long } \\
\text { axes of cylinders of Salem } \\
\text { limestone or Berea sandstone }\end{array}$ & $\begin{array}{l}\text { None dsicusssed; work in } \\
\text { progress }\end{array}$ & Yes & EQ3/EQ6 \\
\hline & \multicolumn{5}{|c|}{ Title of Reference: Leakage of $\mathrm{CO}_{2}$ through Abandoned Wells: Role of Corrosion of Cement } \\
\hline \multirow{2}{*}{ Schulze et al. (2004) } & Fluid-rock experiments & Serpentine & Precipitation of magnesite & None discussed & \\
\hline & \multicolumn{5}{|c|}{ Title of Reference: Characterization of Carbonated Serpentine using XPS and TEM } \\
\hline \multirow{2}{*}{$\begin{array}{l}\text { Shilobreeva and } \\
\text { Martinez (2007) } \\
\text { (abstract only) }\end{array}$} & Fluid-rock experiments & Basalt & None discussed & None discussed & \\
\hline & \multicolumn{5}{|c|}{ Title of Reference: $\mathrm{CO}_{2}$-Water-Basalt Interactions: Experimental and Mineralogical Study } \\
\hline \multirow{2}{*}{$\begin{array}{l}\text { Shiraki and Dunn } \\
(2000)\end{array}$} & Fluid-rock experiments & $\begin{array}{l}\text { Tensleep Formation sandstone, } \\
\text { Wyoming, }\end{array}$ & Dissolution of dolomite cement & Yes & SOLMINEQ.88 \\
\hline & \multicolumn{5}{|c|}{$\begin{array}{l}\text { Title of Reference: Experimental Study on Water-Rock Interactions during } \mathrm{CO}_{2} \text { Flooding in the Tensleep Formation, Wyoming, } \\
\text { USA }\end{array}$} \\
\hline \multirow[t]{2}{*}{ Soong et al. (2004) } & $\begin{array}{l}\text { Fluid-mineral } \\
\text { experiments }\end{array}$ & $\begin{array}{l}\text { Brine samples from the } \\
\text { Oriskany Formation in Indiana } \\
\text { County, Pennsylvania }\end{array}$ & Precipitation of calcite & Yes & PHREEQC \\
\hline & \multicolumn{5}{|c|}{ Title of Reference: Experimental and Simulation Studies on Mineral Trapping of CO2 with Brine } \\
\hline
\end{tabular}


Table 5.4. (contd)

\begin{tabular}{|c|c|c|c|c|c|}
\hline Reference & $\begin{array}{l}\text { Type of Laboratory } \\
\text { Study }\end{array}$ & Sites or Materials Studied & $\begin{array}{c}\text { Carbonate Minerals and } \\
\text { Reactions Physically } \\
\text { Observed }\end{array}$ & $\begin{array}{c}\text { Was } \\
\text { Geochemical } \\
\text { Reaction } \\
\text { Modeling } \\
\text { Used? }\end{array}$ & $\begin{array}{l}\text { Computer } \\
\text { Codes Used for } \\
\text { Geochemical } \\
\text { Reaction } \\
\text { Modeling }\end{array}$ \\
\hline \multirow[t]{2}{*}{$\begin{array}{l}\text { Stefánsson and Gysi } \\
\text { (2008) } \\
\text { (abstract only) }\end{array}$} & Fluid-rock experiments & Basalt & $\begin{array}{l}\mathrm{Mg}-\mathrm{Fe} \text { and } \mathrm{Mg}-\mathrm{Ca} \text { carbonates } \\
\text { form during early low } \mathrm{pH} \\
\text { stage; then with increase in } \mathrm{pH} \text {, } \\
\text { precipitation of calcite } \\
\text { dominates }\end{array}$ & Yes & No details \\
\hline & \multicolumn{5}{|c|}{ Title of Reference: $\mathrm{CO}_{2}$-Basalt Interaction - Numerical Simulation and Experimental Study } \\
\hline \multirow{2}{*}{ Sterpenich et al. (2009); } & Fluid-rock experiments & $\begin{array}{l}\text { Lavoux oolitic limestone from } \\
\text { southwestern area of the Paris } \\
\text { Basin, France }\end{array}$ & $\begin{array}{l}\text { Oolitic limestone consisted of } \\
\text { calcite }\end{array}$ & Yes & CHESS \\
\hline & \multicolumn{5}{|c|}{$\begin{array}{l}\text { Title of Reference: Experimental Ageing of Oolitic Limestones under } \mathrm{CO}_{2} \text { Storage Conditions: Petrographical and Chemical } \\
\text { Evidence }\end{array}$} \\
\hline \multirow[t]{2}{*}{ Stockmann et al. (2008) } & $\begin{array}{l}\text { Fluid-mineral } \\
\text { experiments }\end{array}$ & Diopside and basaltic glass & $\begin{array}{l}\text { Proposed precipitation of } \\
\text { calcite on surfaces of diopside } \\
\text { and basaltic glass particles }\end{array}$ & Yes & PHREEQC \\
\hline & \multicolumn{5}{|c|}{ Title of Reference: Dissolution of Diopside and Basaltic Glass: The Effect of Carbonate Coating } \\
\hline \multirow{2}{*}{$\begin{array}{l}\text { Stockmann et al. (2009) } \\
\text { (abstract only) }\end{array}$} & Fluid-rock experiments & Basaltic glass & Calcite & None discussed & \\
\hline & \multicolumn{5}{|c|}{ Title of Reference: Can Carbonate Coatings Inhibit In Situ Mineral Carbonation? } \\
\hline \multirow{2}{*}{ Suto et al. (2007) } & Fluid-rock experiments & Granite & None discussed & Yes & SOLVEQ \\
\hline & \multicolumn{5}{|c|}{ Title of Reference: Initial Behavior of Granite in Response to Injection of $\mathrm{CO}_{2}$-Saturated Fluid } \\
\hline \multirow{2}{*}{ Teir et al. (2009) } & $\begin{array}{l}\text { Fluid-mineral } \\
\text { experiments }\end{array}$ & Serpentine ore & Hydromagnesite & None discussed & \\
\hline & \multicolumn{5}{|c|}{ Title of Reference: Fixation of Carbon Dioxide by Producing Hydromagnesite from Serpentinite } \\
\hline \multirow{2}{*}{ Testemale et al. (2009) } & $\begin{array}{l}\text { Mineral dissolution } \\
\text { experiments }\end{array}$ & Siderite & None & Yes & CHESS \\
\hline & \multicolumn{5}{|c|}{ Title of Reference: An X-ray Absorption Study of the Dissolution of Siderite at 300 Bar between $50{ }^{\circ} \mathrm{C}$ and $100{ }^{\circ} \mathrm{C}$} \\
\hline
\end{tabular}


Table 5.4. (contd)

\begin{tabular}{|c|c|c|c|c|c|}
\hline Reference & $\begin{array}{l}\text { Type of Laboratory } \\
\text { Study }\end{array}$ & Sites or Materials Studied & $\begin{array}{c}\text { Carbonate Minerals and } \\
\text { Reactions Physically } \\
\text { Observed }\end{array}$ & $\begin{array}{l}\text { Was } \\
\text { Geochemical } \\
\text { Reaction } \\
\text { Modeling } \\
\text { Used? }\end{array}$ & $\begin{array}{c}\text { Computer } \\
\text { Codes Used for } \\
\text { Geochemical } \\
\text { Reaction } \\
\text { Modeling }\end{array}$ \\
\hline \multirow[t]{2}{*}{ Wigand et al. (2008) } & $\begin{array}{l}\text { Fluid-rock } \\
\text { experiments- }\end{array}$ & $\begin{array}{l}\text { Sandstone (lithic arkose) from } \\
\text { Bunter Sandstone Formation }\end{array}$ & $\begin{array}{l}\text { Dissolution of dolomite } \\
\text { cement, but no carbonate } \\
\text { formation was observed }\end{array}$ & Yes & PHREEQC \\
\hline & \multicolumn{5}{|c|}{$\begin{array}{l}\text { Title of Reference: Geochemical Effects of } \mathrm{CO}_{2} \text { Sequestration in Sandstones under Simulated In Situ Conditions of Deep Saline } \\
\text { Aquifers }\end{array}$} \\
\hline \multirow[t]{2}{*}{ Wigand et al. (2009) } & $\begin{array}{l}\text { Fluid-cement/rock } \\
\text { experiments }\end{array}$ & $\begin{array}{l}\text { Composite consisting of the dry } \\
\text { class G Portland well cement } \\
\text { and illite-rich shale of the } \\
\text { Wolfcamp Formation in West } \\
\text { Texas }\end{array}$ & Calcite, aragonite, and vaterite & Yes & $\begin{array}{l}\text { Geochemist's } \\
\text { Workbench }\end{array}$ \\
\hline & \multicolumn{5}{|c|}{ Title of Reference: Geochemical Effects of $\mathrm{CO}_{2}$ Sequestration on Fractured Wellbore Cement at the Cement/Caprock Interface } \\
\hline \multirow[t]{2}{*}{ Wolf et al. (2004) } & Fluid-rock experiments & $\begin{array}{l}\text { Serpentine containing primarily } \\
\text { 1T lizardite with minor clino- } \\
\text { chrysotile }\end{array}$ & Precipitation of magnesite & None discussed & \\
\hline & \multicolumn{5}{|c|}{ Title of Reference: In Situ Observation of $\mathrm{CO}_{2}$ Sequestration Reactions Using a Novel Microreaction System } \\
\hline
\end{tabular}




\subsection{Geochemical Reaction Models and Thermodynamic Databases Used to Study $\mathrm{CO}_{2}$ Sequestration Processes}

\subsection{Computer Models}

The references in Tables 5.1 through 5.4 were first examined to identify the names of any coupled fluid transport and geochemical models and/or dedicated geochemical models (i.e., no coupled fluid transport) that were used in these $\mathrm{CO}_{2}$ sequestration studies. The modeling typically involved the prediction of geochemical changes in fluid and mineral compositions from different scenarios of $\mathrm{CO}_{2}$ injection and sequestration or interpretation of geochemical reactions that occurred in laboratory experiments or at site studies of $\mathrm{CO}_{2}$ sequestration. Table 6.1 lists the names of geochemical reaction models that were used in the studies in Tables 5.1 through 5.4 to evaluate the geochemistry of $\mathrm{CO}_{2}$ sequestration in those systems. The information in Table 6.1 is cross-referenced to the studies in Tables 5.1 through 5.4. When possible, Table 5.4 also includes brief information regarding the main source for the thermodynamic database used for each geochemical reaction model. The purpose of tabulating this information is to identify common sources that had been used for development of their thermodynamic database files. In many, if not most cases, code developers rely on thermodynamic database files taken from well-established geochemical reaction codes and/or published sources of tabulated thermodynamic constants as the principle source(s) for their own thermodynamic databases. 
Table 6.1. Names of Geochemical Reaction Models Used in the Modeling, Site, and Laboratory $\mathrm{CO}_{2}$ Sequestration Studies Listed in Tables 5.1 through 5.4

\begin{tabular}{|c|c|c|c|}
\hline $\begin{array}{l}\text { Computer Code or } \\
\text { Software Package }\end{array}$ & $\begin{array}{c}\text { Thermodynamic } \\
\text { Database Information }\end{array}$ & $\begin{array}{l}\text { Studies that Identify Use } \\
\text { of Computer Code or } \\
\text { Software Package }\end{array}$ & Notes \\
\hline ChemTOUGH & -- & White et al. (2005) & $\begin{array}{l}\text { White et al. (2005) } \\
\text { identified use of } \\
\text { SOLTHERM database } \\
\text { (Reed and Palandri 2006). }\end{array}$ \\
\hline CHEM-TOUGH2 & -- & Pruess et al. (2004) & -- \\
\hline $\begin{array}{l}\text { CHESS } \\
\text { Testemale et al. (2009) }\end{array}$ & -- & $\begin{array}{l}\text { Daval et al. (2009); Dupraz } \\
\text { et al. (2007, 2009b); } \\
\text { Lagneau et al. (2005); } \\
\text { Luquot and Gouze (2009); } \\
\text { Sterpenich et al. (2009); } \\
\text { Testemale et al. (2009) }\end{array}$ & $\begin{array}{l}\text { Dupraz et al. (2009b) cites } \\
\text { van der Lee and Lomenech } \\
\text { (2004) as source of } \\
\text { thermodynamic database. }\end{array}$ \\
\hline CHILLER & $\begin{array}{l}\text { The CHILLER } \\
\text { thermodynamic database } \\
\text { is based on the mineral } \\
\text { data of Holland and } \\
\text { Powell (1998) and } \\
\text { aqueous species data } \\
\text { derived from the } \\
\text { SUPCRT92 database } \\
\text { from Johnson et al. } \\
\text { (1992). }\end{array}$ & $\begin{array}{l}\text { Palandri and Kharaka (2005); } \\
\text { Palandri et al. (2005); } \\
\text { Rosenbauer et al. (2005) }\end{array}$ & -- \\
\hline CRUNCH & $\begin{array}{l}\text { Knauss et al. (2005) } \\
\text { identified use of the } \\
\text { LLNL-EQ3/EQ6 } \\
\text { database. }\end{array}$ & Knauss et al. (2005) & -- \\
\hline DIAPHORE & $\begin{array}{l}\text { Le Gallo et al. (1998) } \\
\text { identified database as } \\
\text { coming from the } \\
\text { KINDISP code as } \\
\text { described in Madé et al. } \\
\text { (1994). }\end{array}$ & $\begin{array}{l}\text { Brosse et al. (2005); Guichet } \\
\text { et al. (2005) }\end{array}$ & $\begin{array}{l}\text { Brosse et al. (2005) list the } \\
\log \mathrm{K}_{\mathrm{r}, 298^{\circ} \text { values used for }} \\
\text { calculations. }\end{array}$ \\
\hline $\begin{array}{l}\text { EQ3/EQ6 } \\
\text { (also includes EQ3NR) }\end{array}$ & $\begin{array}{l}\text { Software has its own } \\
\text { database that is based the } \\
\text { current version of } \\
\text { SUPCRT92 database } \\
\text { (Johnson et al. 1992) and } \\
\text { other sources identified in } \\
\text { database file. }\end{array}$ & $\begin{array}{l}\text { Cipolli et al. (2004); Dilmore } \\
\text { et al. (2008b); Hänchen et al. } \\
\text { (2008); Jarvis et al. (2009); } \\
\text { McGrail et al. (2006); Schaef } \\
\text { and McGrail (2009); Schaef } \\
\text { et al. (2010); Watson et al. } \\
\text { (2004); Zwingmann et al. } \\
\text { (2005) }\end{array}$ & -- \\
\hline FLOTRAN & LLNL-EQ3/EQ6 & $\begin{array}{l}\text { Carey and Lichtner (2007); } \\
\text { Carey et al. (2007); Pruess } \\
\text { et al. (2004) }\end{array}$ & -- \\
\hline
\end{tabular}


Table 6.1. (contd)

\begin{tabular}{|c|c|c|c|}
\hline $\begin{array}{l}\text { Computer Code or } \\
\text { Software Package }\end{array}$ & $\begin{array}{c}\text { Thermodynamic } \\
\text { Database Information }\end{array}$ & $\begin{array}{l}\text { Studies that Identify Use } \\
\text { of Computer Code or } \\
\text { Software Package }\end{array}$ & Notes \\
\hline $\begin{array}{l}\text { GAMSPATH } \\
\text { (This code is the most } \\
\text { recent version of } \\
\text { PATHARC) }\end{array}$ & $\begin{array}{l}\text { The GAMSPATH } \\
\text { thermodynamic database } \\
\text { is based on values } \\
\text { derived from SUPCRT92 } \\
\text { database (Johnson et al. } \\
\text { 1992). }\end{array}$ & $\begin{array}{l}\text { Buschkuehle and Perkins } \\
\text { (2004); Michael and } \\
\text { Buschkuehle (2006); } \\
\text { Palandri and Kharaka (2005) }\end{array}$ & -- \\
\hline GEM-GHG & -- & Ozah et al. (2005) & -- \\
\hline $\begin{array}{l}\text { Geochemist's } \\
\text { Workbench }\end{array}$ & $\begin{array}{l}\text { User may select from } \\
\text { three datasets. These } \\
\text { include those from } \\
\text { LLNL-EQ3/EQ6, } \\
\text { MINTEQ, PHREEQC, } \\
\text { and WATEQ4F codes. }\end{array}$ & $\begin{array}{l}\text { Allen et al. (2005); Baines } \\
\text { and Worden (2004); Moore } \\
\text { et al. (2005); Saylor and } \\
\text { Zerai (2004); Wigand et al. } \\
\text { (2009); Worden (2006); } \\
\text { Zerai et al. (2006) }\end{array}$ & $\begin{array}{l}\text { Baines and Worden } \\
\text { (2004), Moore et al. } \\
\text { (2005), and Worden } \\
\text { (2006) identified the use of } \\
\text { the LLNL-EQ3/EQ6 } \\
\text { database. }\end{array}$ \\
\hline $\begin{array}{l}\text { NUFT-GEMBOCHS- } \\
\text { Xtol toolbox (NUFT) }\end{array}$ & $\begin{array}{l}\text { Johnson et al. (2001) } \\
\text { identified the use of the } \\
\text { most current version of } \\
\text { the SUPCRT92 database } \\
\text { (Johnson et al. 1992) }\end{array}$ & $\begin{array}{l}\text { Johnson et al. (2001); } \\
\text { Johnson et al. (2004) }\end{array}$ & -- \\
\hline PATHARC.94 & & Gunter et al. $(1997 ; 2000)$ & -- \\
\hline PHREEQC & $\begin{array}{l}\text { USGS distributes the } \\
\text { code with three datasets. } \\
\text { These include those from } \\
\text { PHREEQC, WATEQ4F, } \\
\text { and MINTEQ codes. } \\
\text { Others (see last column) } \\
\text { have reported use of } \\
\text { LLNL-EQ3/EQ6 } \\
\text { database with } \\
\text { PHREEQC. }\end{array}$ & $\begin{array}{l}\text { Alemu et al. (2009); } \\
\text { Alfredsson and Gislason } \\
\text { (2009); Allen et al. (2005); } \\
\text { Bateman et al. (2005); } \\
\text { Bertier et al. (2007); } \\
\text { Cantucci et al. (2009b); } \\
\text { Flaathen and Gislason } \\
\text { (2007); Flaathen et al. } \\
\text { (2009); Gaus et al. (2005); } \\
\text { Gysi and Stefánson (2008); } \\
\text { Iglesias et al. (2008); } \\
\text { Kervévan et al. (2005); } \\
\text { Ketzer et al. (2009); May } \\
\text { (2005); Mito et al. (2008); } \\
\text { Mito and Nakagawa (2009); } \\
\text { Pauwels et al. (2007); Sass } \\
\text { et al. (2001); Soong et al. } \\
\text { (2004); Stockmann et al. } \\
\text { (2008); Wigand et al. (2008) }\end{array}$ & $\begin{array}{l}\text { Bateman et al. (2005); } \\
\text { Gaus et al. (2005); Ketzer } \\
\text { et al. (2009); Pauwels } \\
\text { et al. (2007); Sass et al. } \\
\text { (2001); and Wigand et al. } \\
\text { (2008) identify use of } \\
\text { LLNL-EQ3/EQ6 database. } \\
\text { May (2005) used } \\
\text { WATEQ4F database. }\end{array}$ \\
\hline PHRQPITZ & -- & Sass et al. (2001) & -- \\
\hline PRECIP & $\begin{array}{l}\text { Bateman et al. (2005) } \\
\text { identified use of LLNL- } \\
\text { EQ3/EQ6 database. }\end{array}$ & Bateman et al. (2005) & -- \\
\hline SCALE & $\begin{array}{l}\text { Code has its own } \\
\text { database. }\end{array}$ & Pauwels et al. (2007) & -- \\
\hline
\end{tabular}


Table 6.1. (contd)

\begin{tabular}{|c|c|c|c|}
\hline $\begin{array}{c}\text { Computer Code or } \\
\text { Software Package }\end{array}$ & $\begin{array}{c}\text { Thermodynamic } \\
\text { Database Information }\end{array}$ & $\begin{array}{l}\text { Studies that Identify Use } \\
\text { of Computer Code or } \\
\text { Software Package }\end{array}$ & Notes \\
\hline SCALE2000 & $\begin{array}{l}\text { Database based on Pitzer } \\
\text { parameters. Sources of } \\
\text { Pitzer parameters listed in } \\
\text { Azaroual et al. (2004). }\end{array}$ & $\begin{array}{l}\text { André et al. (2007); } \\
\text { Kervévan et al. (2005) }\end{array}$ & -- \\
\hline SOLMINEQ & -- & $\begin{array}{l}\text { Kharaka et al. (2006a, } \\
\text { 2006b); Bachu and Adams } \\
\text { (2003) }\end{array}$ & -- \\
\hline SOLMINDB & -- & Bachu and Adams (2003) & $\begin{array}{l}\text { Modified version of } \\
\text { SOLMINEQ (Bachu and } \\
\text { Adams 2003) }\end{array}$ \\
\hline SOLMINEQ.88 & -- & $\begin{array}{l}\text { Emberley et al. (2005); } \\
\text { Raistrick et al. (2006); } \\
\text { Shiraki and Dunn (2000) }\end{array}$ & $\begin{array}{l}\text { Shiraki and Dunn (2000) } \\
\text { mention corrections to } \\
\text { dolomite }\end{array}$ \\
\hline SOLMINEQ99 & -- & Rosenbauer et al. (2005) & -- \\
\hline SOLVEQ & -- & $\begin{array}{l}\text { Rosenbauer et al. (2005); } \\
\text { Suto et al. (2007) }\end{array}$ & -- \\
\hline STARS & -- & Izgec et al. (2008a, 2008b) & -- \\
\hline TOUGHREACT & $\begin{array}{l}\text { LLNL-EQ3/6 database } \\
\text { with revisions generated } \\
\text { by SUPCRT92. }\end{array}$ & $\begin{array}{l}\text { André et al. (2007); } \\
\text { Audigane et al. (2007); } \\
\text { Cantucci et al. (2009a); } \\
\text { Ennis-King and Paterson } \\
\text { (2007); Gherardi et al. } \\
\text { (2007); Pruess et al. (2004); } \\
\text { Xu et al. (2003, 2004, 2005, } \\
\text { 2006, 2007); Zhang et al. } \\
\text { (2009) }\end{array}$ & $\begin{array}{l}\text { Xu et al. }(2004,2005, \\
\text { 2007) describe in detail } \\
\text { modifications made to } \\
\text { thermodynamic values for } \\
\text { certain carbonate minerals. }\end{array}$ \\
\hline
\end{tabular}

\subsection{Principal Sources Containing Thermodynamic Data for Carbonate Species}

Common sources of thermodynamic databases used with the geochemical reaction models listed in Table 6.1 were reviewed to identify the carbonate gaseous, aqueous, and mineral species and associated thermodynamic data included in those sources. Several published compilations of thermodynamic values that are well known and commonly used by the geosciences community were also reviewed for this information. These databases and published compilations of thermodynamic data include the following:

- Thermodynamic database file for the EQ3/EQ6 geochemical model

- Thermodynamic database file for the MINTEQ2 geochemical model

- Thermodynamic database file for the PHREEQC geochemical model

- Holland and Powell (1998) - An Internally Consistent Thermodynamic Data Set for Phases of Petrological Interest 
- Hummel et al. (2002) - Nagra/PSI Chemical Thermodynamic Data Base 01/01

- Nordstrom et al. (1990) - "Revised Chemical Equilibrium Data for Major Water-Mineral Reactions and Their Limitations" in Chemical Modeling in Aqueous Systems II

- M. H. Reed (University of Oregon, Eugene, Oregon) - SOLTHERM database of equilibria constants (personal communication, January 31, 2008)

- Robie and Hemingway (1995) - Thermodynamic Properties of Minerals and Related Substances at $298.15 \mathrm{~K}$ and 1 bar (10 Pascals) Pressure and at Higher Temperatures (U.S. Geological Survey Bulletin 2131)

- Smith et al. (2004) - NIST Standard Reference Database 46 (NIST Critically Selected Stability Constants of Metal Complexes Database. Version 8 for Windows)

- Woods and Garrels (1987) - Thermodynamic Values at Low Temperature for Natural Inorganic Materials: An Uncritical Summary.

For carbonate species containing elements other than $\mathrm{C}, \mathrm{O}$, and $\mathrm{H}$, we focused our database reviews on thermodynamic data available for carbonate species that contain common alkali and alkaline-earth elements, and $\mathrm{Fe}$ and $\mathrm{Mn}$. It was assumed that these elements would be the most important elements on a concentration basis in aquifer systems, or have the largest potential impact (besides $\mathrm{S}$ ) on redox reactions associated with $\mathrm{CO}_{2}$ sequestration in such systems. The database reviews did not compile information available for carbonate aqueous species and minerals that contain elements, such as $\mathrm{Ba}, \mathrm{Sr}, \mathrm{Cd}, \mathrm{Cu}, \mathrm{Pb}$, $\mathrm{Zn}$, rare earth elements, $\mathrm{Hg}$, and radionuclides such as $\mathrm{U}, \mathrm{Pu}$, and $\mathrm{Am}$.

\subsection{Availability of Thermodynamic Data for $\mathrm{CO}_{2}$ and $\mathrm{CH}_{4}$ Gases and Carbonate-Containing Aqueous Species and Minerals in Identified Databases and Compilations}

Results of our reviews of the thermodynamic databases and published compilations of thermodynamic data listed above are shown in Tables 6.2, 6.3, and 6.4, respectively. The carbonate species and types of thermodynamic data available for each carbonate species are listed in Tables 6.2 through 6.4 according to the reference sources listed above. If a source does not list a particular carbonate species, then that source is not included for that species. Table 6.2 contains thermodynamic data available for pure $\mathrm{CO}_{2}$ and $\mathrm{CH}_{4}$ gases, Table 6.3 contains thermodynamic data available for carbonate aqueous species, and Table 6.4 contains thermodynamic data available for carbonate-containing minerals.

The types of thermodynamic parameters tabulated for compounds vary between the different database sources. Depending on the database, the thermodynamic parameters may include values for the following:

- $\Delta_{\mathrm{f}} \mathrm{G}_{298}{ }^{\circ}$

- $\Delta_{\mathrm{f}} \mathrm{H}_{298}{ }^{\circ}$

- $\mathrm{S}_{298^{\circ}}$

- $\Delta_{\mathrm{r}} \mathrm{H}_{\mathrm{T}}{ }^{\circ}$

- analytical expression for temperature dependence of $\mathrm{C}_{\mathrm{p}}{ }^{\circ}$ at and above $298 \mathrm{~K}$ 
- $\log \mathrm{K}_{\mathrm{r}, 298^{\circ}}$

- analytical expression for temperature dependence of $\log \mathrm{K}_{\mathrm{r}, \mathrm{T}}{ }^{\circ}$

- $\log \mathrm{K}_{\mathrm{r}, \mathrm{T}}{ }^{\circ}$ values estimated or extrapolated at a series of temperatures such as $298 \mathrm{~K}\left(25^{\circ} \mathrm{C}\right), 323 \mathrm{~K}$ $\left(50^{\circ} \mathrm{C}\right), 373 \mathrm{~K}\left(100^{\circ} \mathrm{C}\right), 423 \mathrm{~K}\left(150^{\circ} \mathrm{C}\right), 473 \mathrm{~K}\left(200^{\circ} \mathrm{C}\right), 523 \mathrm{~K}\left(250^{\circ} \mathrm{C}\right), 573 \mathrm{~K}\left(300^{\circ} \mathrm{C}\right)$, and $623 \mathrm{~K}$ $\left(350^{\circ} \mathrm{C}\right)$.

The equations used to derive the $\Delta_{\mathrm{f}} \mathrm{G}_{298}{ }^{\circ}$, and $\log \mathrm{K}_{\mathrm{r}, 298}{ }^{\circ}$ values and $\Delta_{\mathrm{f}} \mathrm{G}_{\mathrm{T}}{ }^{\circ}$, and $\log \mathrm{K}_{\mathrm{r}, \mathrm{T}}{ }^{\circ}$ at higher temperatures are provided in Section 3.0.

Values of $\Delta_{\mathrm{f}} \mathrm{G}_{298}{ }^{\circ}$ and $/$ or $\log \mathrm{K}_{\mathrm{r}, 298^{\circ}}$ were available for essentially all of the compounds in Tables 6.2 through 6.4. $\log \mathrm{K}_{\mathrm{r}, \mathrm{T}}{ }^{\circ}$ or heat capacity values at temperatures above $298 \mathrm{~K}$ were available for less than approximately one-third of these compounds. For those compounds that do not have high-temperature heat capacity data, the tabulated high-temperature $\log \mathrm{K}_{\mathrm{r}, \mathrm{T}}{ }^{\circ}$ are likely based on extrapolation methods that relied on thermodynamic properties reported at $298 \mathrm{~K}\left(25^{\circ} \mathrm{C}\right)$. Because the temperatures of host formations that will be used for $\mathrm{CO}_{2}$ injection and sequestration, and the supporting geochemical reaction modeling studies will range between $50^{\circ} \mathrm{C}$ to $100^{\circ} \mathrm{C}$ or greater, the lack of high-temperature thermodynamic values for key carbonate compounds, especially minerals, will likely impact the accuracy of some modeling calculations.

Values listed in the cited sources for the thermodynamic parameters in Tables 6.2 through 6.4 were not tabulated because this would lead to the necessity of having to compare and explain the differences that exist for thermodynamic values for a particular carbonate compound. To complete an evaluation at that level, one would need to know what the original database manager used for the thermodynamic values for all the ancillary elements and species and in the case of thermodynamic values listed for high temperature, the extrapolation methods to back calculate to the original source values. This is a daunting task, and may not be feasible because records of the calculations and database source values may not have been maintained, sufficiently documented, or be readily available.

The tabulated $\log \mathrm{K}_{\mathrm{r}, 298^{\circ}}$ and $\Delta_{\mathrm{r}} \mathrm{H}_{298}{ }^{\circ}$ values are listed to the same number of significant figures as given in the cited source. The $\log \mathrm{K}_{\mathrm{r}, 298^{\circ}}$ and $\Delta_{\mathrm{r}} \mathrm{H}_{298}{ }^{\circ}$ values are based on dissociation reactions for the specified compounds, which are written in terms of $\mathrm{HCO}_{3}{ }^{-}$and $\mathrm{H}^{+} / \mathrm{H}_{2} \mathrm{O}$. The $\log \mathrm{K}_{\mathrm{r}}, 298^{\circ}$ and $\Delta_{\mathrm{r}} \mathrm{H}_{298}{ }^{\circ}$ values for $\mathrm{CH}_{4}(\mathrm{~g})$ and $\mathrm{CH}_{4}{ }^{\circ}(\mathrm{aq})$ are based on their dissociation reactions written in terms of $\mathrm{HCO}_{3}{ }^{-}$, $\mathrm{H}^{+} / \mathrm{H}_{2} \mathrm{O}$, and $\mathrm{e}^{-}$[not $\left.\mathrm{O}_{2}{ }^{\circ}(\mathrm{aq})\right]$. If different basis species (e.g., $\mathrm{CO}_{3}{ }^{2-}$ ) were used in the cited source, the log $\mathrm{K}_{\mathrm{r}}, 298^{\circ}$ and $\Delta_{\mathrm{r}} \mathrm{H}_{298}{ }^{\circ}$ values given in the source were converted to the $\mathrm{HCO}_{3}{ }^{-}$and $\mathrm{H}^{+} / \mathrm{H}_{2} \mathrm{O}$ basis species using the appropriate $\log \mathrm{K}_{\mathrm{r}}, 298^{\circ}$ and $\Delta_{\mathrm{r}} \mathrm{H}_{298}{ }^{\circ}$ values listed in that same source. 
Table 6.2. Thermodynamic Data Available for $\mathrm{CO}_{2}$ and $\mathrm{CH}_{4}$ Gases

\begin{tabular}{|c|c|c|c|c|c|c|c|}
\hline $\begin{array}{c}\text { Formula for Carbonate } \\
\text { Compound }\end{array}$ & $\begin{array}{c}\Delta_{\mathrm{f}} \mathbf{G}_{\mathbf{2 9 8}}{ }^{\circ} \\
(\mathrm{kJ} / \mathbf{m o l})\end{array}$ & $\begin{array}{c}\Delta_{\mathrm{f}} \mathrm{H}_{298}{ }^{\circ} \\
(\mathrm{kJ} / \mathbf{m o l})\end{array}$ & $\begin{array}{c}\mathrm{S}_{298}^{\circ} \\
(\mathrm{J} / \mathrm{mol} \cdot \mathrm{K})\end{array}$ & $\log K_{r, 298^{\circ}}$ & $\begin{array}{c}\Delta_{\mathrm{r}} \mathbf{H}_{298}{ }^{\circ} \\
(\mathbf{k J} / \mathbf{m o l})\end{array}$ & $\begin{array}{l}\text { Other Values Included } \\
\text { in Source }\end{array}$ & $\begin{array}{l}\text { Listed Values Taken } \\
\text { from Following } \\
\text { Source and } \\
\text { References Therein }\end{array}$ \\
\hline \multirow{5}{*}{$\mathrm{CO}_{2}(\mathrm{~g})$} & -394.30 & -393.51 & 213.70 & -- & -- & $\begin{array}{l}\text { Analytical expression } \\
\text { for temperature } \\
\text { dependence of } C_{p}{ }^{\circ}\end{array}$ & $\begin{array}{l}\text { Holland and Powell } \\
\text { (1998) }\end{array}$ \\
\hline & -394.371 & -393.510 & 213.785 & -7.820 & -10.875 & -- & Hummel et al. (2002) \\
\hline & -- & -- & -- & -7.820 & -10.874 & $\begin{array}{l}\text { Analytical expression } \\
\text { for } \log \mathrm{K}_{\mathrm{r}, \mathrm{T}}{ }^{\circ} \text { as a } \\
\text { function of temperature }\end{array}$ & $\begin{array}{l}\text { Nordstrom et al. } \\
\text { (1990) }\end{array}$ \\
\hline & -- & -- & -- & -7.818 & -- & $\begin{array}{l}\log \mathrm{K}_{\mathrm{r}, \mathrm{T}^{\circ}} \text { at } 298,323, \\
373,423,473,523, \\
573, \text { and } 623 \mathrm{~K}(25,50, \\
100,150,200,250, \\
\left.300, \text { and } 350^{\circ} \mathrm{C}\right) ; \\
\text { analytical expression } \\
\text { for } \log \mathrm{K}_{\mathrm{r}, \mathrm{T}}{ }^{\circ} \text { as a } \\
\text { function of temperature }\end{array}$ & MH Reed ${ }^{(\mathrm{a})}$ \\
\hline & -394.4 & -393.5 & 213.8 & -- & -- & $\begin{array}{l}\text { Analytical expression } \\
\text { for temperature } \\
\text { dependence of } \mathrm{C}_{\mathrm{p}}{ }^{\circ} \text { to } \\
2200 \mathrm{~K}\left(1927^{\circ} \mathrm{C}\right) \text { and } \\
\Delta_{\mathrm{f}} \mathrm{G}_{\mathrm{T}}^{\circ}, \Delta_{\mathrm{f}} \mathrm{H}_{\mathrm{T}}^{\circ}, \text { and } \mathrm{S}_{\mathrm{T}}{ }^{\circ} \\
\text { values up to } 1800 \mathrm{~K} \\
\left(1527^{\circ} \mathrm{C}\right)\end{array}$ & $\begin{array}{l}\text { Robie and } \\
\text { Hemingway (1995) }\end{array}$ \\
\hline
\end{tabular}


Table 6.2. (contd)

\begin{tabular}{|c|c|c|c|c|c|c|c|}
\hline $\begin{array}{c}\text { Formula for Carbonate } \\
\text { Compound }\end{array}$ & $\begin{array}{c}\Delta_{\mathrm{f}} \mathbf{G}_{\mathbf{2 9 8}}{ }^{\circ} \\
(\mathrm{kJ} / \mathbf{m o l})\end{array}$ & $\begin{array}{c}\Delta_{\mathrm{f}} \mathbf{H}_{298}{ }^{\circ} \\
(\mathbf{k J} / \mathbf{m o l})\end{array}$ & $\begin{array}{c}\mathrm{S}_{298}{ }^{\circ} \\
(\mathrm{J} / \mathrm{mol} \cdot \mathrm{K})\end{array}$ & $\log K_{r, 298}{ }^{\circ}$ & $\begin{array}{l}\Delta_{\mathrm{r}} \mathrm{H}_{298}{ }^{\circ} \\
(\mathrm{kJ} / \mathbf{m o l})\end{array}$ & $\begin{array}{l}\text { Other Values Included } \\
\text { in Source }\end{array}$ & $\begin{array}{l}\text { Listed Values Taken } \\
\text { from Following } \\
\text { Source and } \\
\text { References Therein }\end{array}$ \\
\hline \multirow{5}{*}{$\mathrm{CO}_{2}(\mathrm{~g})$} & -- & -- & -- & -7.818 & -10.54 & $\begin{array}{l}\log \mathrm{K}_{\mathrm{r}, \mathrm{T}} \text { listed at one or } \\
\text { more values of } \\
\text { temperature and ionic } \\
\text { strength }\end{array}$ & Smith et al. (2004) \\
\hline & $\begin{array}{l}-394.4 \\
-394.4 \\
-394.4 \\
-394.4 \\
-394.4 \\
-394.4 \\
-394.4 \\
\end{array}$ & $\begin{array}{l}-393.5 \\
-393.5 \\
-393.5 \\
-393.5 \\
-393.5 \\
-393.5 \\
-393.5 \\
\end{array}$ & $\begin{array}{l}213.6 \\
213.6 \\
213.7 \\
213.8 \\
213.7 \\
213.7\end{array}$ & -- & -- & -- & $\begin{array}{l}\text { Woods and Garrels } \\
\text { (1987) }\end{array}$ \\
\hline & -394.36 & -393.51 & 213.74 & -7.8136 & -- & $\begin{array}{l}\log \mathrm{K}_{\mathrm{r}, \mathrm{T}}{ }^{\circ} \text { values also at } \\
273,333,373,423 \text {, } \\
473,523 \text {, and } 573 \mathrm{~K}\end{array}$ & EQ3/EQ6 \\
\hline & -- & -- & -- & -7.820 & -10.874 & $\begin{array}{l}\text { Analytical expression } \\
\text { for } \log \mathrm{K}_{\mathrm{r}, \mathrm{T}}^{\circ} \text { as a } \\
\text { function of temperature }\end{array}$ & PHREEQC \\
\hline & -- & -- & -- & -7.8180 & -10.5400 & -- & MINTEQA2 \\
\hline & & & & & & & \\
\hline \multirow[b]{3}{*}{$\mathrm{CH}_{4}(\mathrm{~g})$} & -50.66 & -74.81 & 186.26 & -- & -- & $\begin{array}{l}\text { Analytical expression } \\
\text { for temperature } \\
\text { dependence of } \mathrm{C}_{\mathrm{p}}{ }^{\circ}\end{array}$ & $\begin{array}{l}\text { Holland and Powell } \\
\text { (1998) }\end{array}$ \\
\hline & -50.721 & -74.810 & -- & -30.705 & 242.085 & -- & Hummel et al. (2002) \\
\hline & -- & -- & -- & -30.686 & -- & $\begin{array}{l}\log \mathrm{K}_{\mathrm{r}, \mathrm{T}^{\circ}} \text { at } 298,323, \\
373,423,473,523, \\
573, \text { and } 623 \mathrm{~K} ; \\
\text { analytical expression } \\
\text { for } \log \mathrm{K}_{\mathrm{r}, \mathrm{T}}{ }^{\circ} \text { as a } \\
\text { function of temperature }\end{array}$ & MH Reed ${ }^{(a)}$ \\
\hline
\end{tabular}


Table 6.2. (contd)

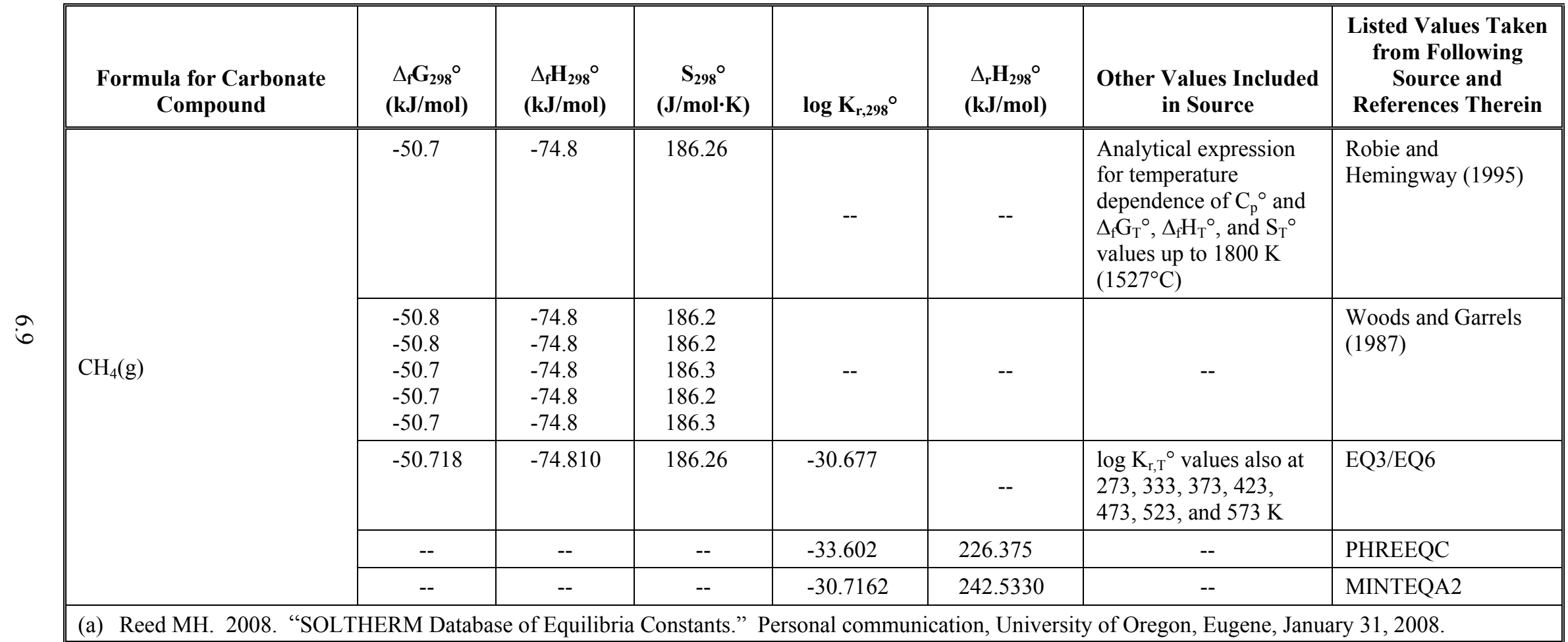


Table 6.3. Thermodynamic Data Available for Aqueous Carbonate Species

\begin{tabular}{|c|c|c|c|c|c|c|c|}
\hline $\begin{array}{c}\text { Formula for Carbonate } \\
\text { Compound }\end{array}$ & $\begin{array}{c}\Delta_{\mathrm{f}} \mathbf{G}_{298}{ }^{\circ} \\
(\mathrm{kJ} / \mathrm{mol})\end{array}$ & $\begin{array}{c}\Delta_{\mathrm{f}} \mathrm{H}_{298}{ }^{\circ} \\
(\mathrm{kJ} / \mathrm{mol})\end{array}$ & $\begin{array}{c}\mathrm{S}_{298}{ }^{\circ} \\
(\mathrm{J} / \mathrm{mol} \cdot \mathrm{K})\end{array}$ & $\log K_{r, 298^{\circ}}$ & $\begin{array}{c}\Delta_{\mathrm{r}} \mathrm{H}_{298}^{\circ} \\
(\mathrm{kJ} / \mathrm{mol})\end{array}$ & $\begin{array}{l}\text { Other Values Included } \\
\text { in Source }\end{array}$ & $\begin{array}{l}\text { Listed Values Taken } \\
\text { from Following } \\
\text { Source and } \\
\text { References Therein }\end{array}$ \\
\hline \multirow{6}{*}{$\mathrm{CO}_{2}{ }^{\circ}(\mathrm{aq})$} & -385.992 & -413.494 & 119.360 & -6.352 & 9.109 & -- & Hummel et al. (2002) \\
\hline & -- & -- & -- & -6.352 & 9.109 & $\begin{array}{l}\text { Analytical expression } \\
\text { for } \log \mathrm{K}_{\mathrm{r}, \mathrm{T}} \mathrm{O} \text { as a } \\
\text { function of temperature }\end{array}$ & $\begin{array}{l}\text { Nordstrom et al. } \\
\text { (1990) }\end{array}$ \\
\hline & -- & -- & -- & -6.345 & -- & $\begin{array}{l}\log \mathrm{K}_{\mathrm{r}, \mathrm{T}}{ }^{\circ} \text { at } 298,323, \\
373,423,473,523, \\
573, \text { and } 623 \mathrm{~K}(25,50, \\
100,150,200,250, \\
\left.300, \text { and } 350^{\circ} \mathrm{C}\right) ; \\
\text { analytical expression } \\
\text { for } \log \mathrm{K}_{\mathrm{r}, \mathrm{O}} \mathrm{O}^{\circ} \text { as a } \\
\text { function of temperature }\end{array}$ & MH Reed $^{(a)}$ \\
\hline & $\begin{array}{l}-386.2 \\
-386.0 \\
-386.0 \\
-386.0\end{array}$ & $\begin{array}{l}-412.9 \\
-413.8 \\
-413.8 \\
-413.8\end{array}$ & $\begin{array}{l}121,3 \\
120.9 \\
117.6 \\
120.9\end{array}$ & -- & -- & -- & $\begin{array}{l}\text { Woods and Garrels } \\
\text { (1987) }\end{array}$ \\
\hline & -385.974 & -413.798 & 117.57 & -6.3447 & -- & $\begin{array}{l}\log \mathrm{K}_{\mathrm{r}, \mathrm{T}^{\circ}} \text { values also at } \\
273,333,373,423, \\
473,523 \text {, and } 573 \mathrm{~K}\end{array}$ & EQ3/EQ6 \\
\hline & -- & -- & -- & -6.352 & 9.110 & $\begin{array}{l}\text { Analytical expression } \\
\text { for } \log \mathrm{K}_{\mathrm{r}, \mathrm{T}}{ }^{\circ} \text { as a } \\
\text { function of temperature }\end{array}$ & PHREEQC \\
\hline
\end{tabular}


Table 6.3. (contd)

\begin{tabular}{|c|c|c|c|c|c|c|c|}
\hline $\begin{array}{c}\text { Formula for Carbonate } \\
\text { Compound }\end{array}$ & $\begin{array}{c}\Delta_{\mathrm{f}} \mathrm{G}_{298}{ }^{\circ} \\
(\mathrm{kJ} / \mathrm{mol})\end{array}$ & $\begin{array}{c}\Delta_{\mathrm{f}} \mathrm{H}_{298}{ }^{\circ} \\
(\mathrm{kJ} / \mathrm{mol})\end{array}$ & $\begin{array}{c}\mathrm{S}_{298}^{\circ} \\
(\mathrm{J} / \mathrm{mol} \cdot \mathrm{K})\end{array}$ & $\log K_{r, 298}^{\circ}$ & $\begin{array}{c}\Delta_{\mathrm{r}} \mathrm{H}_{298}^{\circ} \\
(\mathrm{kJ} / \mathrm{mol})\end{array}$ & $\begin{array}{l}\text { Other Values Included } \\
\text { in Source }\end{array}$ & $\begin{array}{l}\text { Listed Values Taken } \\
\text { from Following } \\
\text { Source and } \\
\text { References Therein }\end{array}$ \\
\hline \multirow{4}{*}{$\mathrm{CH}_{4}{ }^{\circ}(\mathrm{aq})$} & -34.413 & -88.607 & -- & -27.849 & 255.882 & -- & Hummel et al. (2002) \\
\hline & -- & -- & -- & -27.827 & -- & $\begin{array}{l}\log \mathrm{K}_{\mathrm{r}, \mathrm{T}}{ }^{\circ} \text { at } 298,323, \\
373,423,473,523, \\
573, \text { and } 623 \mathrm{~K} ; \\
\text { analytical expression } \\
\text { for } \log \mathrm{K}_{\mathrm{r}, \mathrm{O}}{ }^{\circ} \text { as a } \\
\text { function of temperature }\end{array}$ & MH Reed ${ }^{(\text {a) }}$ \\
\hline & -34.45 & -87.906 & 87.822 & -27.827 & -- & $\begin{array}{l}\log \mathrm{K}_{\mathrm{r}, \mathrm{T}}{ }^{\circ} \text { values also at } \\
273,333,373,423, \\
473,523, \text { and } 573 \mathrm{~K}\end{array}$ & EQ3/EQ6 \\
\hline & -- & -- & -- & -30.742 & 240.49 & -- & PHREEQC \\
\hline & & & & & & & \\
\hline \multirow{5}{*}{$\mathrm{CO}_{3}{ }^{2-}$} & -- & -675.23 & -50.00 & -- & -- & $\mathrm{C}_{\mathrm{p}}^{\circ}$ & $\begin{array}{l}\text { Holland and Powell } \\
\text { (1998) }\end{array}$ \\
\hline & -527.917 & -675.314 & -50.000 & 10.329 & -14.901 & -- & Hummel et al. (2002) \\
\hline & -- & -- & -- & 10.329 & -14.899 & $\begin{array}{l}\text { Analytical expression } \\
\text { for } \log \mathrm{K}_{\mathrm{r}, \mathrm{T}}{ }^{\circ} \text { as a } \\
\text { function of temperature }\end{array}$ & $\begin{array}{l}\text { Nordstrom et al. } \\
\text { (1990) }\end{array}$ \\
\hline & -- & -- & -- & 10.329 & -- & $\begin{array}{l}\log \mathrm{K}_{\mathrm{r}, \mathrm{T}}{ }^{\circ} \text { at } 298,323, \\
373,423,473,523, \\
573, \text { and } 623 \mathrm{~K} ; \\
\text { analytical expression } \\
\text { for } \log \mathrm{K}_{\mathrm{r}, \mathrm{O}} \mathrm{O}^{\circ} \text { as a } \\
\text { function of temperature }\end{array}$ & MH Reed $^{(a)}$ \\
\hline & -527.0 & -675.2 & -50.0 & -- & -- & -- & $\begin{array}{l}\text { Robie and } \\
\text { Hemingway (1995) }\end{array}$ \\
\hline
\end{tabular}


Table 6.3. (contd)

\begin{tabular}{|c|c|c|c|c|c|c|c|}
\hline $\begin{array}{c}\text { Formula for Carbonate } \\
\text { Compound }\end{array}$ & $\begin{array}{l}\Delta_{\mathrm{f}} \mathbf{G}_{298}{ }^{\circ} \\
(\mathrm{kJ} / \mathbf{m o l})\end{array}$ & $\begin{array}{c}\Delta_{\mathrm{f}} \mathbf{H}_{298}^{\circ} \\
(\mathrm{kJ} / \mathbf{m o l})\end{array}$ & $\begin{array}{c}\mathrm{S}_{298}^{\circ} \\
(\mathrm{J} / \mathrm{mol} \cdot \mathrm{K})\end{array}$ & $\log K_{r, 298^{\circ}}$ & $\begin{array}{c}\Delta_{\mathrm{r}} \mathbf{H}_{298}{ }^{\circ} \\
(\mathrm{kJ} / \mathbf{m o l})\end{array}$ & $\begin{array}{l}\text { Other Values Included } \\
\text { in Source }\end{array}$ & $\begin{array}{l}\text { Listed Values Taken } \\
\text { from Following } \\
\text { Source and } \\
\text { References Therein }\end{array}$ \\
\hline \multirow{5}{*}{$\mathrm{CO}_{3}{ }^{2-}$} & -- & -- & -- & 10.329 & -14.6 & $\begin{array}{l}\log \mathrm{K}_{\mathrm{r}, \mathrm{T}} \text { and } \Delta_{\mathrm{r}} \mathrm{H}_{\mathrm{T}} \text { listed } \\
\text { at one or more values of } \\
\text { temperature and ionic } \\
\text { strength }\end{array}$ & $\begin{array}{l}\text { Smith et al. (2004) } \\
\text { (used } \mathrm{CO}_{3}{ }^{--} \text {as basis } \\
\text { species) }\end{array}$ \\
\hline & $\begin{array}{l}-528.1 \\
-527.9 \\
-527.9 \\
-527.8 \\
-527.9 \\
-527.9\end{array}$ & $\begin{array}{l}-676.2 \\
-677.1 \\
-677.1 \\
-677.1 \\
-677.1 \\
-677.1\end{array}$ & $\begin{array}{l}-53.1 \\
-56.9 \\
-56.9 \\
-56.9 \\
-56.9\end{array}$ & -- & -- & -- & $\begin{array}{l}\text { Woods and Garrels } \\
\text { (1987) }\end{array}$ \\
\hline & -527.983 & -675.235 & -49.999 & 10.3288 & -- & $\begin{array}{l}\log \mathrm{K}_{\mathrm{r}, \mathrm{T}^{\circ}} \text { values also at } \\
273,323,373,423 \text {, } \\
473,523 \text {, and } 573 \mathrm{~K}\end{array}$ & EQ3/EQ6 \\
\hline & -- & -- & -- & 10.3290 & -14.6000 & -- & MINTEQA2 \\
\hline & -- & -- & -- & 10.329 & -14.90 & $\begin{array}{l}\text { Analytical expression } \\
\text { for } \log \mathrm{K}_{\mathrm{r}, \mathrm{O}}{ }^{\circ} \text { as a } \\
\text { function of temperature }\end{array}$ & PHREEQC \\
\hline & & & & & & & \\
\hline \multirow{3}{*}{$\mathrm{HCO}_{3}^{-}$} & -586.875 & -690.215 & 98.400 & -- & -- & -- & Hummel et al. (2002) \\
\hline & -- & -- & -- & See $\mathrm{CO}_{3}{ }^{2-}$ & -- & -- & $\begin{array}{l}\text { Nordstrom et al. } \\
\text { (1990) }\end{array}$ \\
\hline & -586.8 & -689.9 & 98.4 & -- & -- & -- & $\begin{array}{l}\text { Robie and } \\
\text { Hemingway (1995) }\end{array}$ \\
\hline
\end{tabular}


Table 6.3. (contd)

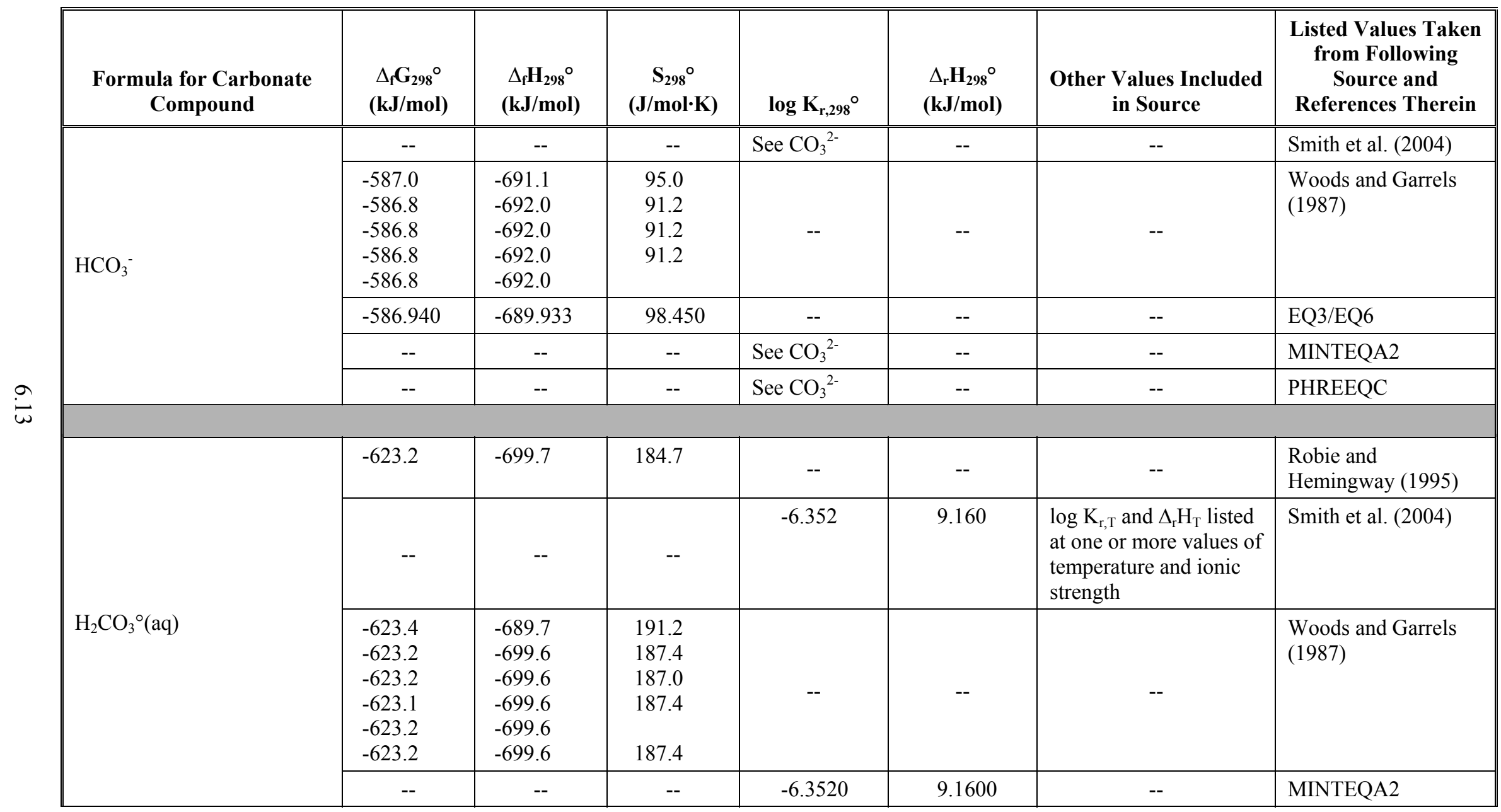


Table 6.3. (contd)

\begin{tabular}{|c|c|c|c|c|c|c|c|}
\hline $\begin{array}{c}\text { Formula for Carbonate } \\
\text { Compound }\end{array}$ & $\begin{array}{c}\Delta_{\mathrm{f}} \mathrm{G}_{298}^{\circ} \\
(\mathrm{kJ} / \mathrm{mol})\end{array}$ & $\begin{array}{c}\Delta_{\mathrm{f}} \mathrm{H}_{298}{ }^{\circ} \\
(\mathrm{kJ} / \mathrm{mol})\end{array}$ & $\begin{array}{c}\mathrm{S}_{298}{ }^{\circ} \\
(\mathrm{J} / \mathrm{mol} \cdot \mathrm{K})\end{array}$ & $\log K_{r, 298}^{\circ}$ & $\begin{array}{c}\Delta_{\mathrm{r}} \mathrm{H}_{298}{ }^{\circ} \\
(\mathrm{kJ} / \mathrm{mol})\end{array}$ & $\begin{array}{l}\text { Other Values Included } \\
\text { in Source }\end{array}$ & $\begin{array}{l}\text { Listed Values Taken } \\
\text { from Following } \\
\text { Source and } \\
\text { References Therein }\end{array}$ \\
\hline \multirow{8}{*}{$\mathrm{CaCO}_{3}{ }^{\circ}(\mathrm{aq})$} & -1099.127 & -1203.482 & $\begin{array}{l}- \\
\end{array}$ & 7.105 & -29.733 & -- & "Hummel et al. (2002) \\
\hline & -- & -- & -- & 7.105 & -29.732 & $\begin{array}{l}\text { Analytical expression } \\
\text { for } \log \mathrm{K}_{\mathrm{r}, \mathrm{T}} \mathrm{o}^{\circ} \text { as a } \\
\text { function of temperature }\end{array}$ & $\begin{array}{l}\text { Nordstrom et al. } \\
\text { (1990) }\end{array}$ \\
\hline & -- & -- & -- & 7.002 & -- & $\begin{array}{l}\log \mathrm{K}_{\mathrm{r}, \mathrm{T}}{ }^{\circ} \text { at } 298,323, \\
373,423,473,523, \\
573, \text { and } 623 \mathrm{~K} ; \\
\text { analytical expression } \\
\text { for } \log \mathrm{K}_{\mathrm{r}, \mathrm{T}} \mathrm{O}^{\circ} \text { as a } \\
\text { function of temperature }\end{array}$ & M. H. Reed ${ }^{(a)}$ \\
\hline & -- & -- & -- & 7.11 & -29.6 & $\begin{array}{l}\log \mathrm{K}_{\mathrm{r}, \mathrm{T}} \text { listed at one or } \\
\text { more values of ionic } \\
\text { strength }\end{array}$ & Smith et al. (2004) \\
\hline & $\begin{array}{l}-1099.4 \\
-1098.9 \\
-1098.9\end{array}$ & -- & -- & -- & -- & -- & $\begin{array}{l}\text { Woods and Garrels } \\
\text { (1987) }\end{array}$ \\
\hline & -1099.76 & -1202.44 & 10.46 & 7.0017 & -- & $\begin{array}{l}\log \mathrm{K}_{\mathrm{r}, \mathrm{T}}{ }^{\circ} \text { values also at } \\
273,333,373,423, \\
473,523, \text { and } 573 \mathrm{~K}\end{array}$ & EQ3/EQ6 \\
\hline & -- & -- & -- & 7.1290 & -30.6000 & -- & MINTEQA2 \\
\hline & -- & -- & -- & 7.105 & -29.730 & $\begin{array}{l}\text { Analytical expression } \\
\text { for } \log \mathrm{K}_{\mathrm{r}, \mathrm{T}}^{\circ} \text { as a } \\
\text { function of temperature }\end{array}$ & PHREEQC \\
\hline
\end{tabular}


Table 6.3. (contd)

\begin{tabular}{|c|c|c|c|c|c|c|c|}
\hline $\begin{array}{c}\text { Formula for Carbonate } \\
\text { Compound }\end{array}$ & $\begin{array}{c}\Delta_{\mathrm{f}} \mathrm{G}_{298}{ }^{\circ} \\
(\mathrm{kJ} / \mathrm{mol})\end{array}$ & $\begin{array}{c}\Delta_{\mathrm{f}} \mathrm{H}_{298}^{\circ} \\
(\mathrm{kJ} / \mathrm{mol})\end{array}$ & $\begin{array}{c}\mathrm{S}_{298}^{\circ} \\
(\mathrm{J} / \mathrm{mol} \cdot \mathrm{K})\end{array}$ & $\log K_{r, 298}^{\circ}$ & $\begin{array}{c}\Delta_{\mathrm{r}} \mathrm{H}_{298}{ }^{\circ} \\
(\mathrm{kJ} / \mathbf{m o l})\end{array}$ & $\begin{array}{l}\text { Other Values Included } \\
\text { in Source }\end{array}$ & $\begin{array}{l}\text { Listed Values Taken } \\
\text { from Following } \\
\text { Source and } \\
\text { References Therein }\end{array}$ \\
\hline \multirow{8}{*}{$\mathrm{CaHCO}_{3}{ }^{+}$} & -1145.992 & -1221.952 & -- & -1.106 & -11.263 & -- & Hummel et al. (2002) \\
\hline & -- & -- & -- & -1.106 & -11.25 & $\begin{array}{l}\text { Analytical expression } \\
\text { for } \log \mathrm{K}_{\mathrm{r}, \mathrm{O}} \text { as a } \\
\text { function of temperature }\end{array}$ & $\begin{array}{l}\text { Nordstrom et al. } \\
(1990)\end{array}$ \\
\hline & -- & -- & -- & -1.047 & -- & $\begin{array}{l}\log \mathrm{K}_{\mathrm{r}, \mathrm{T}}{ }^{\circ} \text { at } 298,323, \\
373,423,473,523, \\
573, \text { and } 623 \mathrm{~K} ; \\
\text { analytical expression } \\
\text { for } \log \mathrm{K}_{\mathrm{r}, \mathrm{O}} \mathrm{O}^{\circ} \text { as a } \\
\text { function of temperature }\end{array}$ & MH Reed ${ }^{(a)}$ \\
\hline & -- & -- & -- & -1.20 & -19.0 & -- & Smith et al. (2004) \\
\hline & -1145.0 & -- & -- & -- & -- & -- & $\begin{array}{l}\text { Woods and Garrels } \\
\text { (1987) }\end{array}$ \\
\hline & -1145.70 & -1231.56 & 66.944 & -1.0467 & -- & $\begin{array}{l}\log \mathrm{K}_{\mathrm{r}, \mathrm{T}}{ }^{\circ} \text { values also at } \\
273,333,373,423, \\
473,523 \text {, and } 573 \mathrm{~K}\end{array}$ & EQ3/EQ6 \\
\hline & -- & -- & -- & -1.2700 & -20.0000 & -- & MINTEQA2 \\
\hline & -- & -- & -- & -1.106 & -11.256 & $\begin{array}{l}\text { Analytical expression } \\
\text { for } \log \mathrm{K}_{\mathrm{r}, \mathrm{T}} \mathrm{O}^{\circ} \text { as a } \\
\text { function of temperature }\end{array}$ & PHREEQC \\
\hline & & & & & & & \\
\hline \multirow{4}{*}{$\mathrm{Fe}^{\mathrm{II}} \mathrm{CO}_{3}{ }^{\circ}(\mathrm{aq})$} & -631.818 & -- & -- & 5.949 & -- & -- & Hummel et al. (2002) \\
\hline & -- & -- & -- & 5.95 & -- & -- & $\begin{array}{l}\text { Nordstrom et al. } \\
(1990)\end{array}$ \\
\hline & -646.487 & -- & -- & 5.5988 & -- & -- & EQ3/EQ6 \\
\hline & -- & -- & -- & 5.949 & -- & -- & PHREEQC \\
\hline
\end{tabular}


Table 6.3. (contd)

\begin{tabular}{|c|c|c|c|c|c|c|c|}
\hline $\begin{array}{c}\text { Formula for Carbonate } \\
\text { Compound }\end{array}$ & $\begin{array}{c}\Delta_{\mathrm{f}} \mathbf{G}_{298}{ }^{\circ} \\
(\mathrm{kJ} / \mathbf{m o l})\end{array}$ & $\begin{array}{c}\Delta_{\mathrm{f}} \mathbf{H}_{298}{ }^{\circ} \\
(\mathrm{kJ} / \mathbf{m o l})\end{array}$ & $\begin{array}{c}\mathrm{S}_{298}^{\circ} \\
(\mathrm{J} / \mathrm{mol} \cdot \mathrm{K})\end{array}$ & $\log K_{r, 298^{\circ}}$ & $\begin{array}{c}\Delta_{\mathrm{r}} \mathrm{H}_{298}{ }^{\circ} \\
(\mathrm{kJ} / \mathrm{mol})\end{array}$ & $\begin{array}{l}\text { Other Values Included } \\
\text { in Source }\end{array}$ & $\begin{array}{l}\text { Listed Values Taken } \\
\text { from Following } \\
\text { Source and } \\
\text { References Therein }\end{array}$ \\
\hline $\mathrm{Fe}^{\mathrm{III}} \mathrm{CO}_{3}^{+}$ & -600.701 & -789.722 & -358.569 & 0.6088 & -- & $\begin{array}{l}\log \mathrm{K}_{\mathrm{r}, \mathrm{T}}{ }^{\circ} \text { values also at } \\
273,333,373,423, \\
473,523 \text {, and } 573 \mathrm{~K}\end{array}$ & EQ3/EQ6 \\
\hline & & & & & & & \\
\hline \multirow{6}{*}{$\mathrm{Fe}^{\mathrm{II}} \mathrm{HCO}_{3}^{+}$} & -677.191 & -- & -- & -2.000 & -- & -- & Hummel et al. (2002) \\
\hline & -- & -- & -- & -2.000 & -- & -- & $\begin{array}{l}\text { Nordstrom et al. } \\
(1990)\end{array}$ \\
\hline & -- & -- & -- & -1.10 & -- & -- & Smith et al. (2004) \\
\hline & -693.971 & -- & -- & -2.7200 & -- & -- & EQ3/EQ6 \\
\hline & -- & -- & -- & -1.1000 & -- & -- & MINTEQA2 \\
\hline & -- & -- & -- & -2.0 & -- & -- & PHREEQC \\
\hline & & & & & & & \\
\hline \multirow{4}{*}{$\mathrm{MgCO}_{3}{ }^{\circ}(\mathrm{aq})$} & -1000.300 & -1130.963 & -- & 7.349 & -26.252 & -- & Hummel et al. (2002) \\
\hline & -- & -- & -- & 7.35 & -26.250 & $\begin{array}{l}\text { Analytical expression } \\
\text { for } \log \mathrm{K}_{\mathrm{r}, \mathrm{T}}{ }^{\circ} \text { as a } \\
\text { function of temperature }\end{array}$ & $\begin{array}{l}\text { Nordstrom et al. } \\
\text { (1990) }\end{array}$ \\
\hline & -- & -- & -- & 7.350 & -- & $\begin{array}{l}\log \mathrm{K}_{\mathrm{r}, \mathrm{T}}{ }^{\circ} \text { at } 298,323, \\
373,423,473,523, \\
573, \text { and } 623 \mathrm{~K} ; \\
\text { analytical expression } \\
\text { for } \log \mathrm{K}_{\mathrm{r}, \mathrm{O}} \mathrm{O}^{\circ} \text { as a } \\
\text { function of temperature }\end{array}$ & MH Reed $^{(a)}$ \\
\hline & -- & -- & -- & 7.41 & -24.6 & $\begin{array}{l}\log \mathrm{K}_{\mathrm{r}, \mathrm{T}} \text { listed at one or } \\
\text { more values of ionic } \\
\text { strength }\end{array}$ & Smith et al. (2004) \\
\hline
\end{tabular}


Table 6.3. (contd)

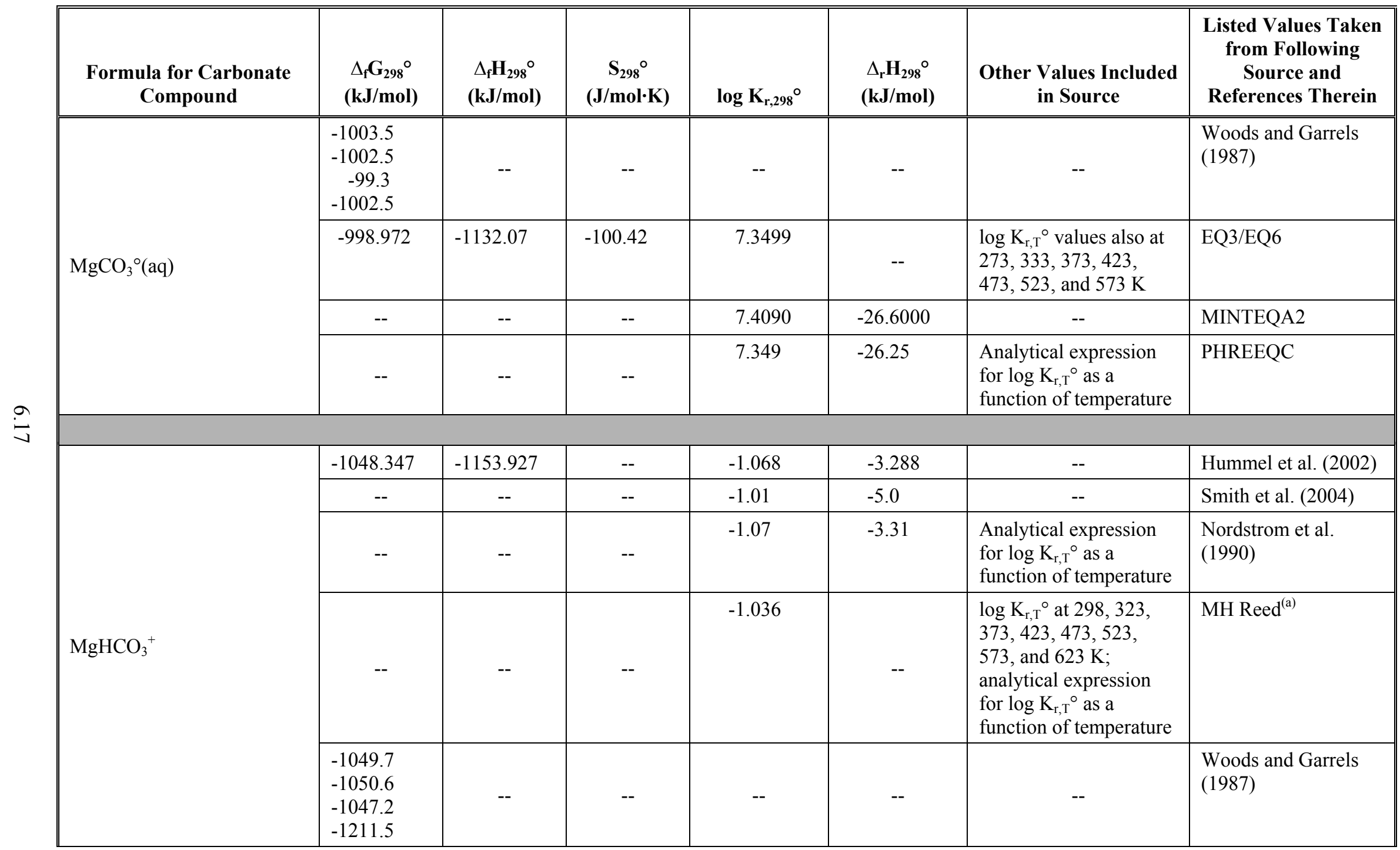


Table 6.3. (contd)

\begin{tabular}{|c|c|c|c|c|c|c|c|}
\hline $\begin{array}{c}\text { Formula for Carbonate } \\
\text { Compound }\end{array}$ & $\begin{array}{c}\Delta_{\mathrm{f}} \mathbf{G}_{298}{ }^{\circ} \\
(\mathrm{kJ} / \mathrm{mol})\end{array}$ & $\begin{array}{c}\Delta_{\mathrm{f}} \mathrm{H}_{298}{ }^{\circ} \\
(\mathrm{kJ} / \mathrm{mol})\end{array}$ & $\begin{array}{c}\mathrm{S}_{298}{ }^{\circ} \\
(\mathrm{J} / \mathrm{mol} \cdot \mathrm{K})\end{array}$ & $\log K_{r, 298^{\circ}}$ & $\begin{array}{c}\Delta_{\mathrm{r}} \mathrm{H}_{298}{ }^{\circ} \\
(\mathrm{kJ} / \mathrm{mol})\end{array}$ & $\begin{array}{l}\text { Other Values Included } \\
\text { in Source }\end{array}$ & $\begin{array}{l}\text { Listed Values Taken } \\
\text { from Following } \\
\text { Source and } \\
\text { References Therein }\end{array}$ \\
\hline \multirow{3}{*}{$\mathrm{MgHCO}_{3}^{+}$} & -1046.84 & -1153.74 & -12.55 & -1.0357 & -- & $\begin{array}{l}\log \mathrm{K}_{\mathrm{r}, \mathrm{T}^{\circ}} \text { values also at } \\
273,333,373,423 \text {, } \\
473,523 \text {, and } 573 \mathrm{~K}\end{array}$ & EQ3/EQ6 \\
\hline & -- & -- & -- & -1.0100 & -4.0000 & -- & MINTEQA2 \\
\hline & -- & -- & -- & -1.070 & -3.310 & $\begin{array}{l}\text { Analytical expression } \\
\text { for } \log \mathrm{K}_{\mathrm{r}, \mathrm{O}} \mathrm{O} \text { as a } \\
\text { function of temperature }\end{array}$ & PHREEQC \\
\hline & & & & & & & \\
\hline \multirow{7}{*}{$\mathrm{MnCO}_{3}{ }^{\circ}(\mathrm{aq})$} & -783.986 & -- & -- & 5.429 & -- & -- & Hummel et al. (2002) \\
\hline & -- & -- & -- & 5.43 & -- & -- & $\begin{array}{l}\text { Nordstrom et al. } \\
(1990)\end{array}$ \\
\hline & -- & -- & -- & 3.820 & -- & $\begin{array}{l}\log \mathrm{K}_{\mathrm{r}, \mathrm{T}}{ }^{\circ} \text { at } 298,323, \\
373,423,473,523, \\
573, \text { and } 623 \mathrm{~K} ; \\
\text { analytical expression } \\
\text { for } \log \mathrm{K}_{\mathrm{r}, \mathrm{T}} \mathrm{O}^{\circ} \text { as a } \\
\text { function of temperature }\end{array}$ & M. H. Reed ${ }^{(a)}$ \\
\hline & -- & -- & -- & 5.63 & -- & $\begin{array}{l}\log \mathrm{K}_{\mathrm{r}, \mathrm{T}} \text { listed at one or } \\
\text { more values of ionic } \\
\text { strength }\end{array}$ & Smith et al. (2004) \\
\hline & -751.4 & -895.0 & -136.8 & -- & -- & -- & $\begin{array}{l}\text { Woods and Garrels } \\
\text { (1987) }\end{array}$ \\
\hline & -781.810 & -- & -- & 5.8088 & -- & -- & EQ3/EQ6 \\
\hline & -- & -- & -- & 5.429 & -- & -- & PHREEQC \\
\hline
\end{tabular}


Table 6.3. (contd)

\begin{tabular}{|c|c|c|c|c|c|c|c|}
\hline $\begin{array}{c}\text { Formula for Carbonate } \\
\text { Compound }\end{array}$ & $\begin{array}{c}\Delta_{\mathrm{f}} \mathbf{G}_{298}{ }^{\circ} \\
(\mathrm{kJ} / \mathbf{m o l})\end{array}$ & $\begin{array}{c}\Delta_{\mathrm{f}} \mathrm{H}_{298}{ }^{\circ} \\
(\mathrm{kJ} / \mathrm{mol})\end{array}$ & $\begin{array}{c}\mathrm{S}_{298}^{\circ} \\
(\mathrm{J} / \mathrm{mol} \cdot \mathrm{K})\end{array}$ & $\log K_{r, 298}^{\circ}$ & $\begin{array}{c}\Delta_{\mathrm{r}} \mathrm{H}_{298}{ }^{\circ} \\
(\mathrm{kJ} / \mathrm{mol})\end{array}$ & $\begin{array}{l}\text { Other Values Included } \\
\text { in Source }\end{array}$ & $\begin{array}{l}\text { Listed Values Taken } \\
\text { from Following } \\
\text { Source and } \\
\text { References Therein }\end{array}$ \\
\hline \multirow{7}{*}{$\mathrm{MnHCO}_{3}^{+}$} & -826.106 & -- & -- & -1.950 & -- & -- & Hummel et al. (2002) \\
\hline & -- & -- & -- & -1.95 & -- & -- & $\begin{array}{l}\text { Nordstrom et al. } \\
\text { (1990) }\end{array}$ \\
\hline & -- & -- & -- & -1.30 & -4.0 & $\begin{array}{l}\log \mathrm{K}_{\mathrm{r}, \mathrm{T}} \text { listed at one or } \\
\text { more values of ionic } \\
\text { strength }\end{array}$ & Smith et al. (2004) \\
\hline & -820 & -- & -- & -- & -- & -- & $\begin{array}{l}\text { Woods and Garrels } \\
\text { (1987) }\end{array}$ \\
\hline & -820.000 & -- & -- & -0.8816 & -- & -- & EQ3/EQ6 \\
\hline & -- & -- & -- & -1.3000 & -4.0000 & -- & MINTEQA2 \\
\hline & -- & -- & -- & -1.950 & -- & -- & PHREEQC \\
\hline & & & & & & & \\
\hline \multirow{5}{*}{$\mathrm{NaCO}_{3}^{-}$} & -797.119 & -878.375 & -- & 9.059 & -52.180 & -- & Hummel et al. (2002) \\
\hline & -- & -- & -- & 9.06 & -52.179 & -- & $\begin{array}{l}\text { Nordstrom et al. } \\
(1990)\end{array}$ \\
\hline & -- & -- & -- & 9.360 & -- & $\begin{array}{l}\log \mathrm{K}_{\mathrm{r}, \mathrm{T}}{ }^{\circ} \text { at } 298,323, \\
373,423,473,523, \\
573, \text { and } 623 \mathrm{~K} ; \\
\text { analytical expression } \\
\text { for } \log \mathrm{K}_{\mathrm{r}, \mathrm{T}} \mathrm{o}^{\circ} \text { as a } \\
\text { function of temperature }\end{array}$ & M. H. Reed ${ }^{(\mathrm{a})}$ \\
\hline & -- & -- & -- & 9.06 & -- & $\begin{array}{l}\log \mathrm{K}_{\mathrm{r}, \mathrm{T}} \text { listed at one or } \\
\text { more values of ionic } \\
\text { strength }\end{array}$ & Smith et al. (2004) \\
\hline & $\begin{array}{l}-797.2 \\
-792.8\end{array}$ & -935.9 & -49.8 & -- & -- & -- & $\begin{array}{l}\text { Woods and Garrels } \\
\text { (1987) }\end{array}$ \\
\hline
\end{tabular}


Table 6.3. (contd)

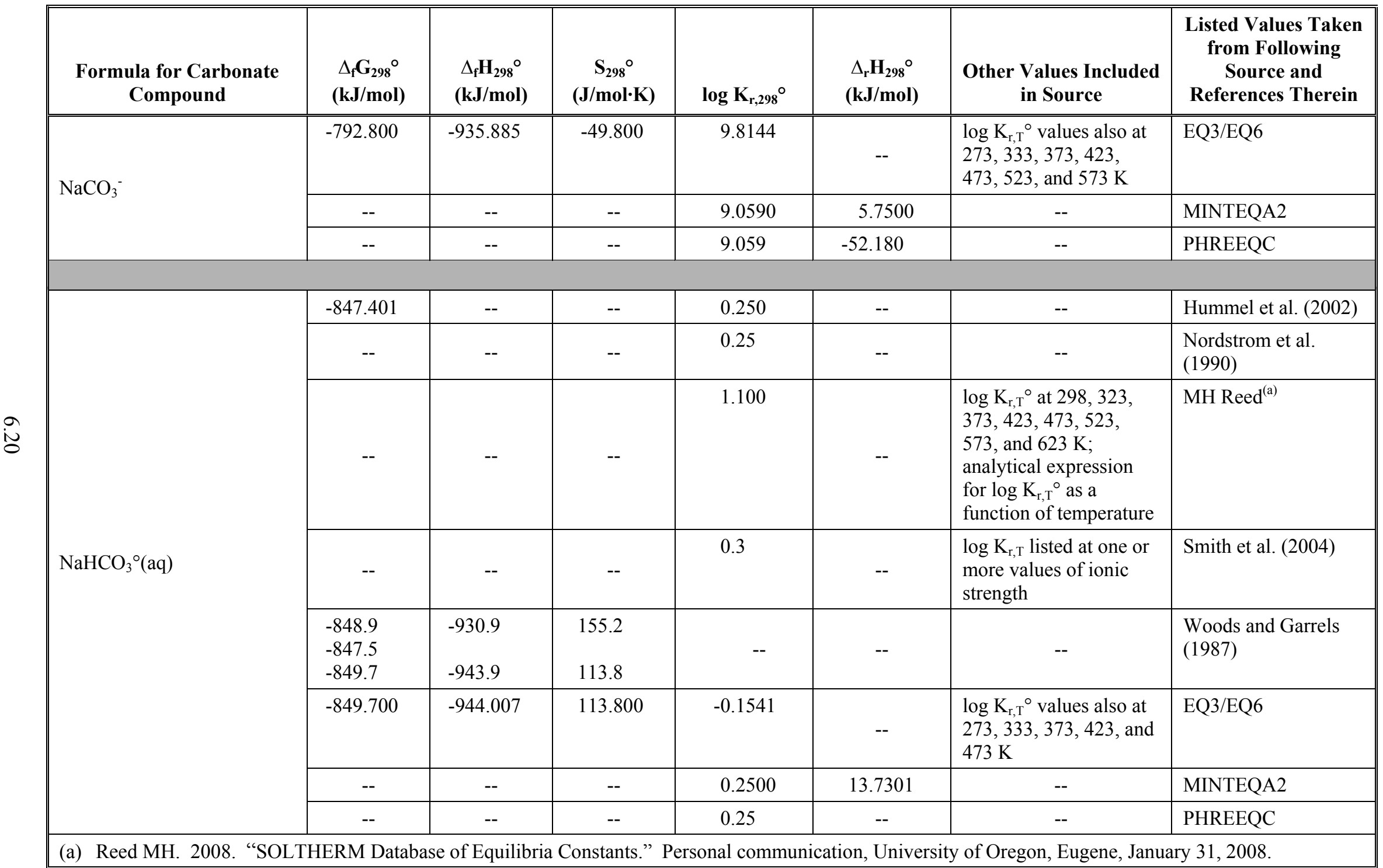


Table 6.4. Thermodynamic Data Available for Carbonate-Containing Minerals

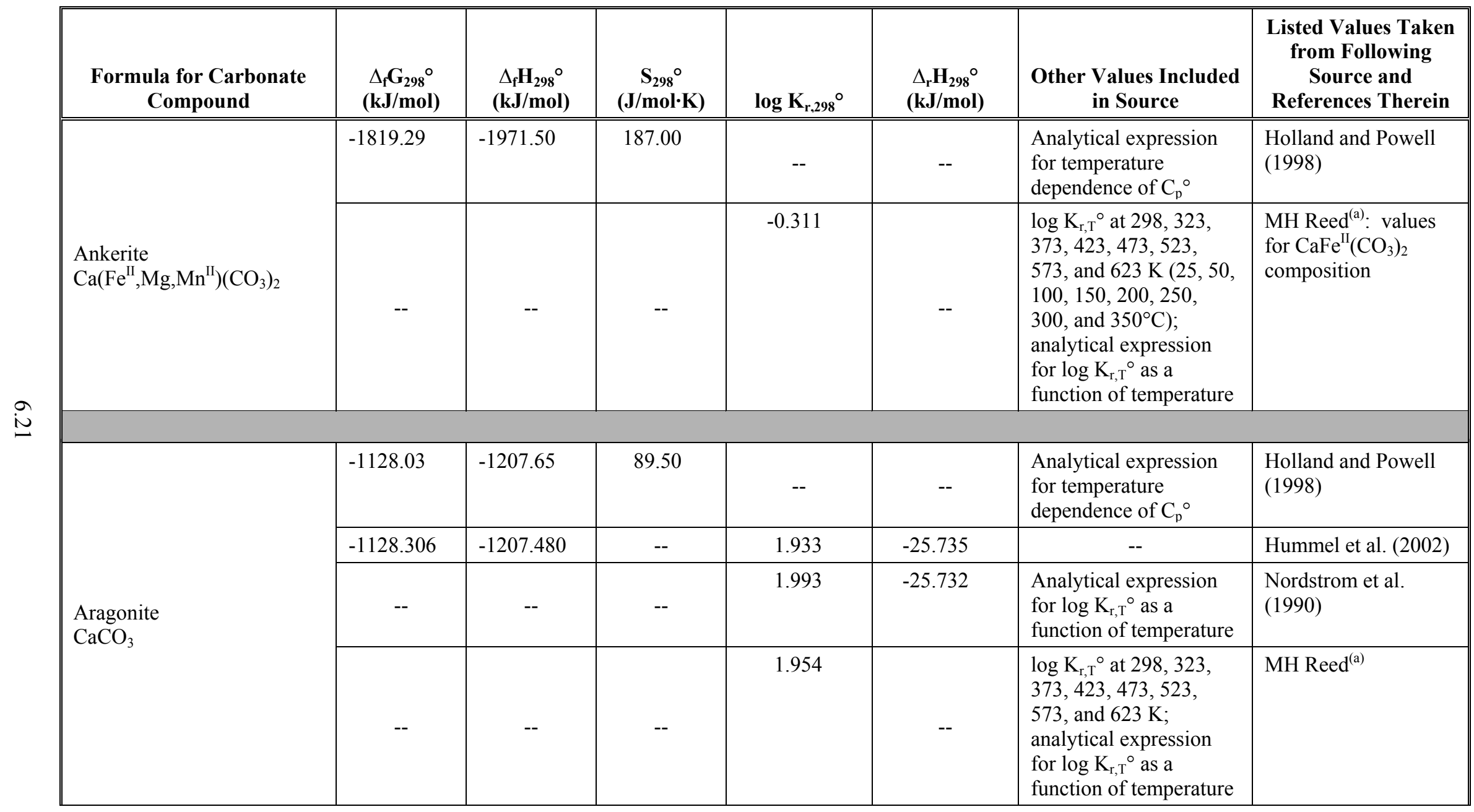


Table 6.4. (contd)

\begin{tabular}{|c|c|c|c|c|c|c|c|}
\hline $\begin{array}{c}\text { Formula for Carbonate } \\
\text { Compound }\end{array}$ & $\begin{array}{l}\Delta_{\mathbf{f}} \mathbf{G}_{298}{ }^{\circ} \\
(\mathbf{k J} / \mathbf{m o l})\end{array}$ & $\begin{array}{c}\Delta_{\mathrm{f}} \mathbf{H}_{298}^{\circ} \\
(\mathbf{k J} / \mathbf{m o l})\end{array}$ & $\begin{array}{c}\mathbf{S}_{298}^{\circ}{ }^{\circ} \\
(\mathrm{J} / \mathbf{m o l} \cdot \mathrm{K})\end{array}$ & $\log K_{r, 298^{\circ}}$ & $\begin{array}{c}\Delta_{\mathrm{r}} \mathbf{H}_{298}^{\circ} \\
(\mathbf{k J} / \mathbf{m o l})\end{array}$ & $\begin{array}{c}\text { Other Values Included } \\
\text { in Source }\end{array}$ & $\begin{array}{l}\text { Listed Values Taken } \\
\text { from Following } \\
\text { Source and } \\
\text { References Therein }\end{array}$ \\
\hline \multirow{6}{*}{$\begin{array}{l}\text { Aragonite } \\
\mathrm{CaCO}_{3}\end{array}$} & -1127.4 & -1207.4 & 88.0 & -- & -- & $\begin{array}{l}\text { Analytical expression } \\
\text { for temperature } \\
\text { dependence of } \mathrm{C}_{\mathrm{p}}^{\circ} \text { and } \\
\Delta_{\mathrm{f}} \mathrm{G}_{\mathrm{T}}^{\circ}, \Delta_{\mathrm{f}} \mathrm{H}_{\mathrm{T}}{ }^{\circ}, \text { and } \mathrm{S}_{\mathrm{T}}{ }^{\circ} \\
\text { values up to } 1000 \mathrm{~K} \\
\left(727^{\circ} \mathrm{C}\right)\end{array}$ & $\begin{array}{l}\text { Robie and } \\
\text { Hemingway (1995) }\end{array}$ \\
\hline & -- & -- & -- & 2.02 & -24.6 & -- & Smith et al. (2004) \\
\hline & $\begin{array}{l}-1127.7 \\
-1127.4 \\
-1128.5 \\
-1127.8 \\
-1129.2 \\
-1127.8 \\
-1128.2 \\
-1129.4 \\
-1130.1\end{array}$ & $\begin{array}{l}-1207.0 \\
-1207.0 \\
-1207.4 \\
-1208.0 \\
-1207.1 \\
-1209.7\end{array}$ & $\begin{array}{l}88.7 \\
88.0 \\
88.7 \\
88.0 \\
90.2 \\
88.7 \\
\\
87.9\end{array}$ & -- & -- & -- & $\begin{array}{l}\text { Woods and Garrels } \\
\text { (1987) }\end{array}$ \\
\hline & -1128.35 & -1207.21 & 90.207 & 1.9931 & -- & $\begin{array}{l}\log \mathrm{K}_{\mathrm{r},{ }^{\circ}} \text { values also at } \\
273,333,373,423 \text {, } \\
473,523 \text {, and } 573 \mathrm{~K}\end{array}$ & EQ3/EQ6 \\
\hline & -- & -- & -- & 2.0290 & -26.6000 & -- & MINTEQA2 \\
\hline & -- & -- & -- & 1.993 & -25.730 & $\begin{array}{l}\text { Analytical expression } \\
\text { for } \log \mathrm{K}_{\mathrm{r}, \mathrm{O}} \mathrm{O}^{\circ} \text { as a } \\
\text { function of temperature }\end{array}$ & PHREEQC \\
\hline
\end{tabular}


Table 6.4. (contd)

\begin{tabular}{|c|c|c|c|c|c|c|c|}
\hline $\begin{array}{c}\text { Formula for Carbonate } \\
\text { Compound }\end{array}$ & $\begin{array}{c}\Delta_{\mathrm{f}} \mathbf{G}_{\mathbf{2 9 8}}{ }^{\circ} \\
(\mathrm{kJ} / \mathbf{m o l}) \\
\end{array}$ & $\begin{array}{c}\Delta_{\mathrm{f}} \mathrm{H}_{298}{ }^{\circ} \\
(\mathrm{kJ} / \mathbf{m o l}) \\
\end{array}$ & $\begin{array}{c}\mathrm{S}_{298}{ }^{\circ} \\
(\mathrm{J} / \mathrm{mol} \cdot \mathrm{K}) \\
\end{array}$ & $\log K_{r, 298^{\circ}}$ & $\begin{array}{c}\Delta_{\mathrm{r}} \mathbf{H}_{298}{ }^{\circ} \\
(\mathbf{k J} / \mathbf{m o l}) \\
\end{array}$ & $\begin{array}{l}\text { Other Values Included } \\
\text { in Source }\end{array}$ & $\begin{array}{l}\text { Listed Values Taken } \\
\text { from Following } \\
\text { Source and } \\
\text { References Therein } \\
\end{array}$ \\
\hline \multirow{5}{*}{$\begin{array}{l}\text { Artinite } \\
\mathrm{Mg}_{2} \mathrm{CO}_{3}(\mathrm{OH})_{2} \cdot 3 \mathrm{H}_{2} \mathrm{O}\end{array}$} & -- & -- & -- & 19.656 & -- & $\begin{array}{l}\log \mathrm{K}_{\mathrm{r}, \mathrm{T}^{\circ}} \text { at } 298,323, \\
373,423,473,523, \\
573, \text { and } 623 \mathrm{~K} ; \\
\text { analytical expression } \\
\text { for } \log \mathrm{K}_{\mathrm{r}, \mathrm{T}}{ }^{\circ} \text { as a } \\
\text { function of temperature }\end{array}$ & MH Reed $^{(a)}$ \\
\hline & -2568.4 & -2920.6 & 232.9 & -- & -- & -- & $\begin{array}{l}\text { Robie and } \\
\text { Hemingway (1995) }\end{array}$ \\
\hline & $\begin{array}{l}-2568.3 \\
-2568.6\end{array}$ & $\begin{array}{l}-2920.6 \\
-2920.6 \\
\end{array}$ & $\begin{array}{l}232.9 \\
232.9\end{array}$ & -- & -- & -- & $\begin{array}{l}\text { Woods and Garrels } \\
\text { (1987) }\end{array}$ \\
\hline & -2568.62 & -2920.61 & 232.92 & 19.6560 & -- & $\begin{array}{l}\log \mathrm{K}_{\mathrm{r}, \mathrm{T}}{ }^{\circ} \text { values also at } \\
273,333,373,423 \text {, } \\
473,523 \text {, and } 573 \mathrm{~K}\end{array}$ & EQ3/EQ6 \\
\hline & -- & -- & -- & 19.9290 & -134.8565 & -- & MINTEQA2 \\
\hline $\begin{array}{l}\text { Burkeite } \\
\mathrm{Na}_{6} \mathrm{CO}_{3}\left(\mathrm{SO}_{4}\right)_{2}\end{array}$ & -3592.99 & -- & -- & 9.4866 & -- & -- & EQ3/EQ6 \\
\hline \multirow{3}{*}{$\begin{array}{l}\text { Calcite } \\
\mathrm{CaCO}_{3}\end{array}$} & -1128.81 & -1207.54 & 92.50 & -- & -- & $\begin{array}{l}\text { Analytical expression } \\
\text { for temperature } \\
\text { dependence of } C_{p}{ }^{\circ}\end{array}$ & $\begin{array}{l}\text { Holland and Powell } \\
\text { (1998) }\end{array}$ \\
\hline & -1129.127 & -1208.705 & -- & 1.849 & -24.510 & -- & Hummel et al. (2002) \\
\hline & -- & -- & -- & 1.849 & -24.510 & $\begin{array}{l}\text { Analytical expression } \\
\text { for } \log \mathrm{K}_{\mathrm{r}, \mathrm{T}}^{\circ} \text { as a } \\
\text { function of temperature }\end{array}$ & $\begin{array}{l}\text { Nordstrom et al. } \\
(1990)\end{array}$ \\
\hline
\end{tabular}


Table 6.4. (contd)

\begin{tabular}{|c|c|c|c|c|c|c|c|}
\hline $\begin{array}{c}\text { Formula for Carbonate } \\
\text { Compound }\end{array}$ & $\begin{array}{l}\Delta_{\mathrm{f}} \mathbf{G}_{298}{ }^{\circ} \\
(\mathbf{k J} / \mathbf{m o l})\end{array}$ & $\begin{array}{c}\Delta_{\mathrm{f}} \mathbf{H}_{298}^{\circ} \\
(\mathrm{kJ} / \mathbf{m o l})\end{array}$ & $\begin{array}{c}\mathrm{S}_{298}^{\circ}{ }^{\circ} \\
(\mathrm{J} / \mathbf{m o l} \cdot \mathbf{K})\end{array}$ & $\log K_{r, 298^{\circ}}$ & $\begin{array}{c}\Delta_{\mathrm{r}} \mathrm{H}_{298}^{\circ} \\
(\mathrm{kJ} / \mathbf{m o l})\end{array}$ & $\begin{array}{c}\text { Other Values Included } \\
\text { in Source }\end{array}$ & $\begin{array}{l}\text { Listed Values Taken } \\
\text { from Following } \\
\text { Source and } \\
\text { References Therein }\end{array}$ \\
\hline \multirow{5}{*}{$\begin{array}{l}\text { Calcite } \\
\mathrm{CaCO}_{3}\end{array}$} & -- & -- & -- & 1.816 & -- & $\begin{array}{l}\log \mathrm{K}_{\mathrm{r}, \mathrm{T}}{ }^{\circ} \text { at } 298,323, \\
373,423,473,523, \\
573 \text {, and } 623 \mathrm{~K} ; \\
\text { analytical expression } \\
\text { for } \log \mathrm{K}_{\mathrm{r}, \mathrm{O}} \mathrm{o}^{\circ} \text { as a } \\
\text { function of temperature }\end{array}$ & M. H. Reed ${ }^{(a)}$ \\
\hline & -1128.5 & -1207.4 & 91.7 & -- & -- & $\begin{array}{l}\text { Analytical expression } \\
\text { for temperature } \\
\text { dependence of } \mathrm{C}_{\mathrm{p}}{ }^{\circ} \text { and } \\
\Delta_{\mathrm{f}} \mathrm{G}_{\mathrm{T}}^{\circ}, \Delta_{\mathrm{f}} \mathrm{H}_{\mathrm{T}}^{\circ} \text {, and } \mathrm{S}_{\mathrm{T}}^{\circ} \\
\text { values up to } 1200 \mathrm{~K} \\
\left(927^{\circ} \mathrm{C}\right)\end{array}$ & $\begin{array}{l}\text { Robie and } \\
\text { Hemingway (1995) }\end{array}$ \\
\hline & -- & -- & -- & 1.85 & -24.6 & $\begin{array}{l}\log \mathrm{K}_{\mathrm{r}, \mathrm{T}} \text { listed at one or } \\
\text { more values of ionic } \\
\text { strength }\end{array}$ & Smith et al. (2004) \\
\hline & $\begin{array}{l}-1128.8 \\
-1128.3 \\
-1128.8 \\
-1130.1 \\
-1128.8 \\
-1128.8 \\
-1129.3 \\
-1130.3 \\
-1130.6\end{array}$ & $\begin{array}{l}-1206.9 \\
-1206.8 \\
-1207.7 \\
-1207.4 \\
-1208.2 \\
-1206.9 \\
-1207.4 \\
-1209.0\end{array}$ & $\begin{array}{l}92.9 \\
91.7 \\
92.9 \\
91.7 \\
92.7 \\
92.9 \\
\\
\\
91.8\end{array}$ & -- & -- & -- & $\begin{array}{l}\text { Woods and Garrels } \\
\text { (1987) }\end{array}$ \\
\hline & -1129.18 & -1207.30 & 92.676 & 1.8487 & -- & $\begin{array}{l}\log \mathrm{K}_{\mathrm{r}, \mathrm{T}^{\circ}} \text { values also at } \\
273,333,373,423 \text {, } \\
473,523 \text {, and } 573 \mathrm{~K}\end{array}$ & EQ3/EQ6 \\
\hline
\end{tabular}


Table 6.4. (contd)

\begin{tabular}{|c|c|c|c|c|c|c|c|}
\hline $\begin{array}{c}\text { Formula for Carbonate } \\
\text { Compound }\end{array}$ & $\begin{array}{c}\Delta_{\mathrm{f}} \mathbf{G}_{298}{ }^{\circ} \\
(\mathbf{k J} / \mathbf{m o l})\end{array}$ & $\begin{array}{c}\Delta_{\mathrm{f}} \mathrm{H}_{298}^{\circ} \\
(\mathrm{kJ} / \mathbf{m o l})\end{array}$ & $\begin{array}{c}\mathrm{S}_{298}^{\circ} \\
(\mathrm{J} / \mathrm{mol} \cdot \mathrm{K})\end{array}$ & $\log K_{r, 298}^{\circ}$ & $\begin{array}{c}\Delta_{\mathrm{r}} \mathrm{H}_{298}^{\circ} \\
(\mathbf{k J} / \mathbf{m o l})\end{array}$ & $\begin{array}{l}\text { Other Values Included } \\
\text { in Source }\end{array}$ & $\begin{array}{l}\text { Listed Values Taken } \\
\text { from Following } \\
\text { Source and } \\
\text { References Therein }\end{array}$ \\
\hline \multirow[b]{2}{*}{$\begin{array}{l}\text { Calcite } \\
\mathrm{CaCO}_{3}\end{array}$} & -- & -- & -- & 1.8490 & -22.6000 & -- & MINTEQA2 \\
\hline & -- & -- & -- & 1.849 & -24.52 & $\begin{array}{l}\text { Analytical expression } \\
\text { for } \log \mathrm{K}_{\mathrm{r}, \mathrm{O}}^{\circ} \text { as a } \\
\text { function of temperature }\end{array}$ & PHREEQC \\
\hline $\mathrm{CaCO}_{3}$ (amorphous solid) & -- & -- & -- & 3.94 & -31.6 & -- & Smith et al. (2004) \\
\hline \multirow{5}{*}{$\begin{array}{l}\text { Dawsonite } \\
\mathrm{NaAlCO}_{3}(\mathrm{OH})_{2}\end{array}$} & -1782 & -1960 & 131 & -- & -- & -- & Bénézeth et al. (2007) \\
\hline & -- & -- & -- & 3.660 & -- & $\begin{array}{l}\log \mathrm{K}_{\mathrm{r}, \mathrm{T}}{ }^{\circ} \text { at } 298,323, \\
373,423,473,523, \\
573, \text { and } 623 \mathrm{~K} ; \\
\text { analytical expression } \\
\text { for } \log \mathrm{K}_{\mathrm{r}, \mathrm{T}}{ }^{\circ} \text { as a } \\
\text { function of temperature }\end{array}$ & MH Reed $^{(a)}$ \\
\hline & -1786.0 & -1964.0 & 132.0 & -- & -- & $\begin{array}{l}\text { Analytical expression } \\
\text { for temperature } \\
\text { dependence of } \mathrm{C}_{\mathrm{p}}{ }^{\circ} \text { and } \\
\Delta_{\mathrm{f}} \mathrm{G}_{\mathrm{T}}{ }^{\circ}, \Delta_{\mathrm{f}} \mathrm{H}_{\mathrm{T}}{ }^{\circ} \text {, and } \mathrm{S}_{\mathrm{T}}{ }^{\circ} \\
\text { values up to } 500 \mathrm{~K} \\
\left(227^{\circ} \mathrm{C}\right)\end{array}$ & $\begin{array}{l}\text { Robie and } \\
\text { Hemingway (1995) }\end{array}$ \\
\hline & -1786.0 & -1964.0 & 132.0 & -- & -- & -- & $\begin{array}{l}\text { Woods and Garrels } \\
\text { (1987) }\end{array}$ \\
\hline & -1785.990 & -1963.956 & 132.000 & 4.3464 & -- & $\begin{array}{l}\log \mathrm{K}_{\mathrm{r}, \mathrm{T}}{ }^{\circ} \text { values also at } \\
273,333,373,423 \text {, and } \\
473 \mathrm{~K}\end{array}$ & EQ3/EQ6 \\
\hline
\end{tabular}


Table 6.4. (contd)

\begin{tabular}{|c|c|c|c|c|c|c|c|}
\hline $\begin{array}{c}\text { Formula for Carbonate } \\
\text { Compound }\end{array}$ & $\begin{array}{l}\Delta_{\mathrm{f}} \mathbf{G}_{298}{ }^{\circ} \\
(\mathbf{k J} / \mathbf{m o l})\end{array}$ & $\begin{array}{c}\Delta_{\mathrm{f}} \mathbf{H}_{298}^{\circ} \\
(\mathrm{kJ} / \mathbf{m o l})\end{array}$ & $\begin{array}{c}\mathrm{S}_{298}^{\circ} \\
(\mathrm{J} / \mathbf{m o l} \cdot \mathbf{K})\end{array}$ & $\log K_{r, 298^{\circ}}$ & $\begin{array}{c}\Delta_{\mathrm{r}} \mathbf{H}_{298}{ }^{\circ} \\
(\mathrm{kJ} / \mathbf{m o l})\end{array}$ & $\begin{array}{l}\text { Other Values Included } \\
\text { in Source }\end{array}$ & $\begin{array}{l}\text { Listed Values Taken } \\
\text { from Following } \\
\text { Source and } \\
\text { References Therein }\end{array}$ \\
\hline \multirow{6}{*}{$\begin{array}{l}\text { Dolomite (ordered) } \\
\mathrm{CaMg}\left(\mathrm{CO}_{3}\right)_{2}\end{array}$} & -2161.51 & -2324.56 & 156.00 & -- & -- & $\begin{array}{l}\text { Analytical expression } \\
\text { for temperature } \\
\text { dependence of } \mathrm{C}_{\mathrm{p}}{ }^{\circ}\end{array}$ & $\begin{array}{l}\text { Holland and Powell } \\
\text { (1998) }\end{array}$ \\
\hline & -2161.565 & -2321.148 & -- & 3.568 & -69.282 & -- & Hummel et al. (2002) \\
\hline & -- & -- & -- & 3.57 & -69.279 & -- & $\begin{array}{l}\text { Nordstrom et al. } \\
\text { (1990) }\end{array}$ \\
\hline & -- & -- & -- & 3.240 & -- & $\begin{array}{l}\log \mathrm{K}_{\mathrm{r}, \mathrm{T}}{ }^{\circ} \text { at } 298,323, \\
373,423,473,523, \\
573, \text { and } 623 \mathrm{~K} \\
\text { analytical expression } \\
\text { for } \log \mathrm{K}_{\mathrm{r}, \mathrm{T}} \mathrm{O}^{\circ} \text { as a } \\
\text { function of temperature }\end{array}$ & MH Reed ${ }^{(a)}$ \\
\hline & -2161.3 & -2324.5 & 155.2 & -- & -- & $\begin{array}{l}\text { Analytical expression } \\
\text { for temperature } \\
\text { dependence of } \mathrm{C}_{\mathrm{p}}{ }^{\circ} \text { and } \\
\Delta_{\mathrm{f}} \mathrm{G}_{\mathrm{T}}^{\circ}, \Delta_{\mathrm{f}} \mathrm{H}_{\mathrm{T}}{ }^{\circ}, \text { and } \mathrm{S}_{\mathrm{T}}{ }^{\circ} \\
\text { values up to } 1100 \mathrm{~K} \\
\left(827^{\circ} \mathrm{C}\right)\end{array}$ & $\begin{array}{l}\text { Robie and } \\
\text { Hemingway (1995) }\end{array}$ \\
\hline & $\begin{array}{l}-2169.3 \\
-2151.9 \\
-2170.0 \\
-2161.7 \\
-2167.2 \\
-2163.4 \\
-2177.8\end{array}$ & $\begin{array}{l}-2331.7 \\
-2314.6 \\
-2332.7 \\
-2324.5 \\
-2329.9 \\
-2326.3\end{array}$ & $\begin{array}{l}155.2 \\
155.2 \\
155.2 \\
155.2 \\
155.2\end{array}$ & -- & -- & -- & $\begin{array}{l}\text { Woods and Garrels } \\
\text { (1987) }\end{array}$ \\
\hline
\end{tabular}


Table 6.4. (contd)

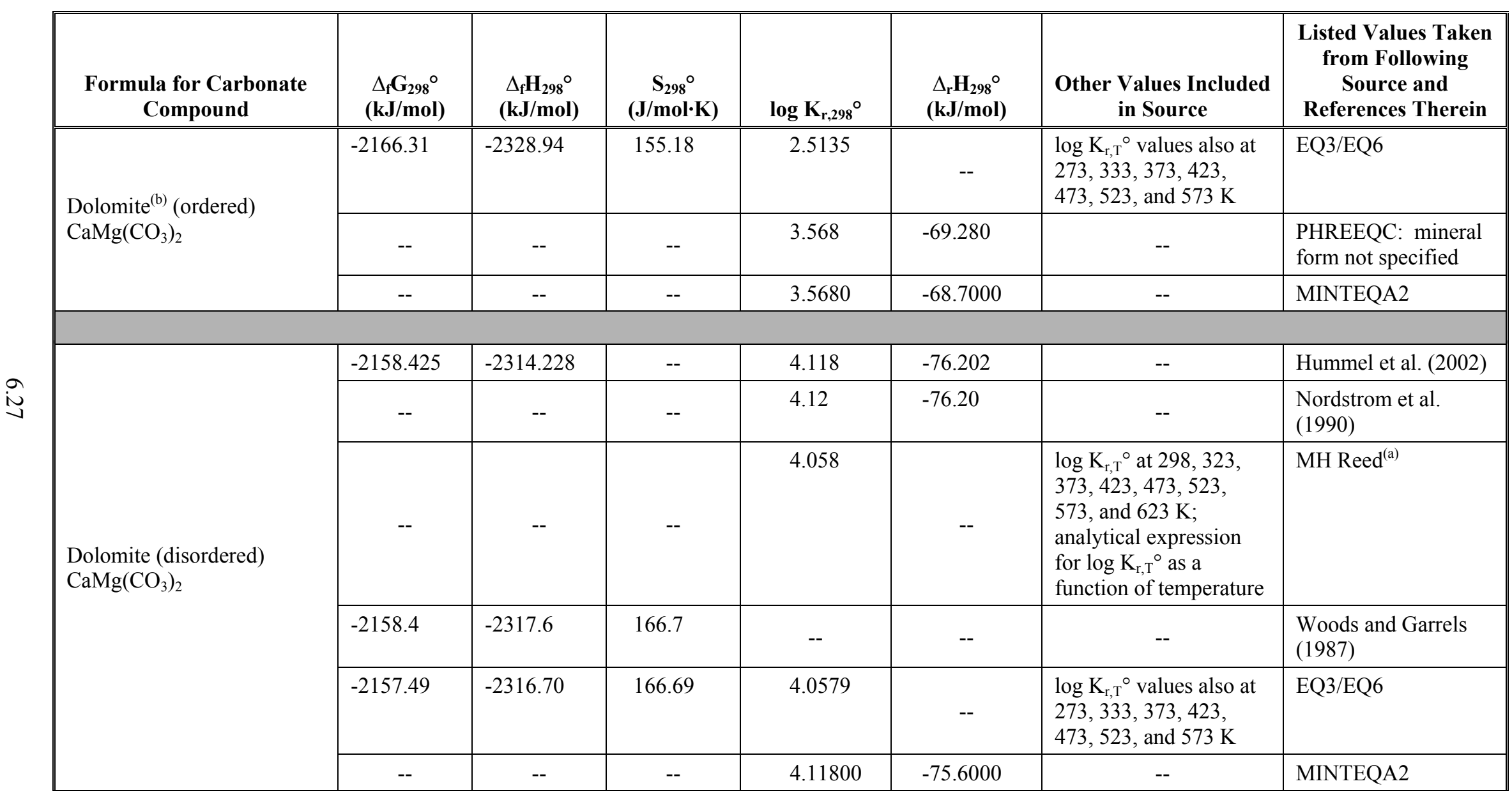


Table 6.4. (contd)

\begin{tabular}{|c|c|c|c|c|c|c|c|}
\hline $\begin{array}{c}\text { Formula for Carbonate } \\
\text { Compound }\end{array}$ & $\begin{array}{c}\Delta_{\mathrm{f}} \mathbf{G}_{298}^{\circ} \\
(\mathrm{kJ} / \mathbf{m o l})\end{array}$ & $\begin{array}{c}\Delta_{\mathrm{f}} \mathbf{H}_{298}{ }^{\circ} \\
(\mathbf{k J} / \mathbf{m o l})\end{array}$ & $\begin{array}{c}\mathrm{S}_{298}{ }^{\circ} \\
(\mathrm{J} / \mathrm{mol} \cdot \mathrm{K})\end{array}$ & $\log K_{r, 298^{\circ}}$ & $\begin{array}{c}\Delta_{\mathrm{r}} \mathbf{H}_{298}^{\circ} \\
(\mathrm{kJ} / \mathbf{m o l})\end{array}$ & $\begin{array}{l}\text { Other Values Included } \\
\text { in Source }\end{array}$ & $\begin{array}{l}\text { Listed Values Taken } \\
\text { from Following } \\
\text { Source and } \\
\text { References Therein }\end{array}$ \\
\hline $\begin{array}{l}\text { Gaylussite } \\
\mathrm{CaNa}_{2}\left(\mathrm{CO}_{3}\right)_{2} \cdot 5 \mathrm{H}_{2} \mathrm{O}\end{array}$ & -3372.61 & -- & -- & 11.1641 & -- & -- & EQ3/EQ6 \\
\hline \multirow{5}{*}{$\begin{array}{l}\text { Huntite } \\
\mathrm{CaMg}_{3}\left(\mathrm{CO}_{3}\right)_{4}\end{array}$} & -- & -- & -- & 10.301 & -- & $\begin{array}{l}\log \mathrm{K}_{\mathrm{r}, \mathrm{T}}{ }^{\circ} \text { at } 298,323, \\
373,423,473,523, \\
573 \text {, and } 623 \mathrm{~K} \text {; } \\
\text { analytical expression } \\
\text { for } \log \mathrm{K}_{\mathrm{r}, \mathrm{O}}{ }^{\circ} \text { as a } \\
\text { function of temperature }\end{array}$ & M. H. Reed ${ }^{(a)}$ \\
\hline & -4203.1 & -4529.6 & 299.5 & -- & -- & -- & $\begin{array}{l}\text { Robie and } \\
\text { Hemingway (1995) }\end{array}$ \\
\hline & $\begin{array}{l}-4203.4 \\
-4216.2 \\
\end{array}$ & -4529.6 & 299.5 & -- & -- & -- & $\begin{array}{l}\text { Woods and Garrels } \\
\text { (1987) }\end{array}$ \\
\hline & -4203.71 & -4529.60 & 299.53 & 10.3010 & -- & $\begin{array}{l}\log \mathrm{K}_{\mathrm{r}, \mathrm{T}}{ }^{\circ} \text { values also at } \\
273,333,373,423, \\
473,523 \text {, and } 573 \mathrm{~K}\end{array}$ & EQ3/EQ6 \\
\hline & -- & -- & -- & 11.3480 & -166.1798 & -- & MINTEQA2 \\
\hline $\mathrm{Mg}_{4}\left(\mathrm{CO}_{3}\right)_{3}(\mathrm{OH})_{2} \cdot 3 \mathrm{H}_{2} \mathrm{O}$ & $\begin{array}{l}-4603.3 \\
-4637.1 \\
\end{array}$ & -- & -- & -- & -- & -- & $\begin{array}{l}\text { Woods and Garrels } \\
\text { (1987) }\end{array}$ \\
\hline & & & & & & & \\
\hline \multirow[t]{2}{*}{$\begin{array}{l}\text { Hydromagnesite } \\
\mathrm{Mg}_{5}\left(\mathrm{CO}_{3}\right)_{4}(\mathrm{OH})_{2} \cdot 4 \mathrm{H}_{2} \mathrm{O}\end{array}$} & -- & -- & -- & 30.854 & -- & $\begin{array}{l}\log \mathrm{K}_{\mathrm{r}, \mathrm{T}}{ }^{\circ} \text { at } 298,323, \\
373,423,473,523, \\
573, \text { and } 623 \mathrm{~K} ; \\
\text { analytical expression } \\
\text { for } \log \mathrm{K}_{\mathrm{r}, \mathrm{T}} \mathrm{o}^{\circ} \text { as a } \\
\text { function of temperature }\end{array}$ & MH Reed ${ }^{(a)}$ \\
\hline & -5864.2 & -6514.9 & 503.7 & -- & -- & -- & $\begin{array}{l}\text { Robie and } \\
\text { Hemingway (1995) }\end{array}$ \\
\hline
\end{tabular}


Table 6.4. (contd)

\begin{tabular}{|c|c|c|c|c|c|c|c|}
\hline $\begin{array}{c}\text { Formula for Carbonate } \\
\text { Compound }\end{array}$ & $\begin{array}{c}\Delta_{\mathrm{f}} \mathbf{G}_{298}^{\circ} \\
(\mathrm{kJ} / \mathbf{m o l})\end{array}$ & $\begin{array}{c}\Delta_{\mathrm{f}} \mathrm{H}_{298}{ }^{\circ} \\
(\mathrm{kJ} / \mathbf{m o l})\end{array}$ & $\begin{array}{c}\mathrm{S}_{298}^{\circ}{ }^{\circ} \\
(\mathrm{J} / \mathbf{m o l} \cdot \mathbf{K})\end{array}$ & $\log K_{r, 298^{\circ}}$ & $\begin{array}{c}\Delta_{\mathrm{r}} \mathbf{H}_{298}{ }^{\circ} \\
(\mathbf{k J} / \mathbf{m o l})\end{array}$ & $\begin{array}{l}\text { Other Values Included } \\
\text { in Source }\end{array}$ & $\begin{array}{l}\text { Listed Values Taken } \\
\text { from Following } \\
\text { Source and } \\
\text { References Therein }\end{array}$ \\
\hline \multirow{3}{*}{$\begin{array}{l}\text { Hydromagnesite } \\
\mathrm{Mg}_{5}\left(\mathrm{CO}_{3}\right)_{4}(\mathrm{OH})_{2} \cdot 4 \mathrm{H}_{2} \mathrm{O}\end{array}$} & $\begin{array}{l}-5864.2 \\
-5864.6\end{array}$ & $\begin{array}{l}-6514.9 \\
-6514.9\end{array}$ & $\begin{array}{l}503.7 \\
541.3\end{array}$ & -- & -- & -- & $\begin{array}{l}\text { Woods and Garrels } \\
\text { (1987) }\end{array}$ \\
\hline & -5864.66 & -6514.86 & 541.33 & 30.8539 & -- & $\begin{array}{l}\log \mathrm{K}_{\mathrm{r}, \mathrm{T}}{ }^{\circ} \text { values also at } \\
273,333,373,423 \text {, } \\
473,523 \text {, and } 573 \mathrm{~K}\end{array}$ & EQ3/EQ6 \\
\hline & -- & -- & -- & 32.5500 & -276.8466 & -- & MINTEQA2 \\
\hline & & & & & & & \\
\hline \multirow{2}{*}{$\begin{array}{l}\text { Ikaite } \\
\mathrm{CaCO}_{3} \cdot 6 \mathrm{H}_{2} \mathrm{O}\end{array}$} & -2540.9 & -2954.1 & 370.0 & -- & -- & -- & $\begin{array}{l}\text { Robie and } \\
\text { Hemingway (1995): } \\
\Delta_{\mathrm{f}} \mathrm{G}_{298^{\circ}}{ }^{\circ} \Delta_{\mathrm{f}} \mathrm{H}_{298^{\circ}}, \mathrm{S}_{298^{\circ}}\end{array}$ \\
\hline & -- & -- & -- & 2.87 & -39.6 & -- & $\begin{array}{l}\text { Smith et al. (2004) } \\
\text { (only composition, } \\
\text { but not mineral form, } \\
\text { specified) }\end{array}$ \\
\hline $\mathrm{K}_{2} \mathrm{CO}_{3} \cdot 1.5 \mathrm{H}_{2} \mathrm{O}$ & -1431.27 & -- & -- & 13.3785 & -- & -- & EQ3/EQ6 \\
\hline $\mathrm{K}_{8} \mathrm{H}_{4}\left(\mathrm{CO}_{3}\right)_{6} \cdot 3 \mathrm{H}_{2} \mathrm{O}$ & -1514.032 & -- & -- & 27.7099 & -- & -- & EQ3/EQ6 \\
\hline $\mathrm{KNaCO}_{3} \cdot 6 \mathrm{H}_{2} \mathrm{O}$ & -596.513 & -- & -- & 10.2593 & -- & -- & EQ3/EQ6 \\
\hline $\mathrm{K}_{2} \mathrm{CO}_{3}$ & $\begin{array}{l}-1069.0 \\
-1064.0 \\
-1060.6\end{array}$ & $\begin{array}{l}-1146.1 \\
-1149.8\end{array}$ & $\begin{array}{l}140.6 \\
155.5\end{array}$ & -- & -- & -- & $\begin{array}{l}\text { Woods and Garrels } \\
\text { (1987) }\end{array}$ \\
\hline $\begin{array}{l}\text { Kalicinite } \\
\mathrm{KHCO}_{3}\end{array}$ & -207.405 & -- & -- & 0.2837 & -- & -- & EQ3/EQ6 \\
\hline
\end{tabular}


Table 6.4. (contd)

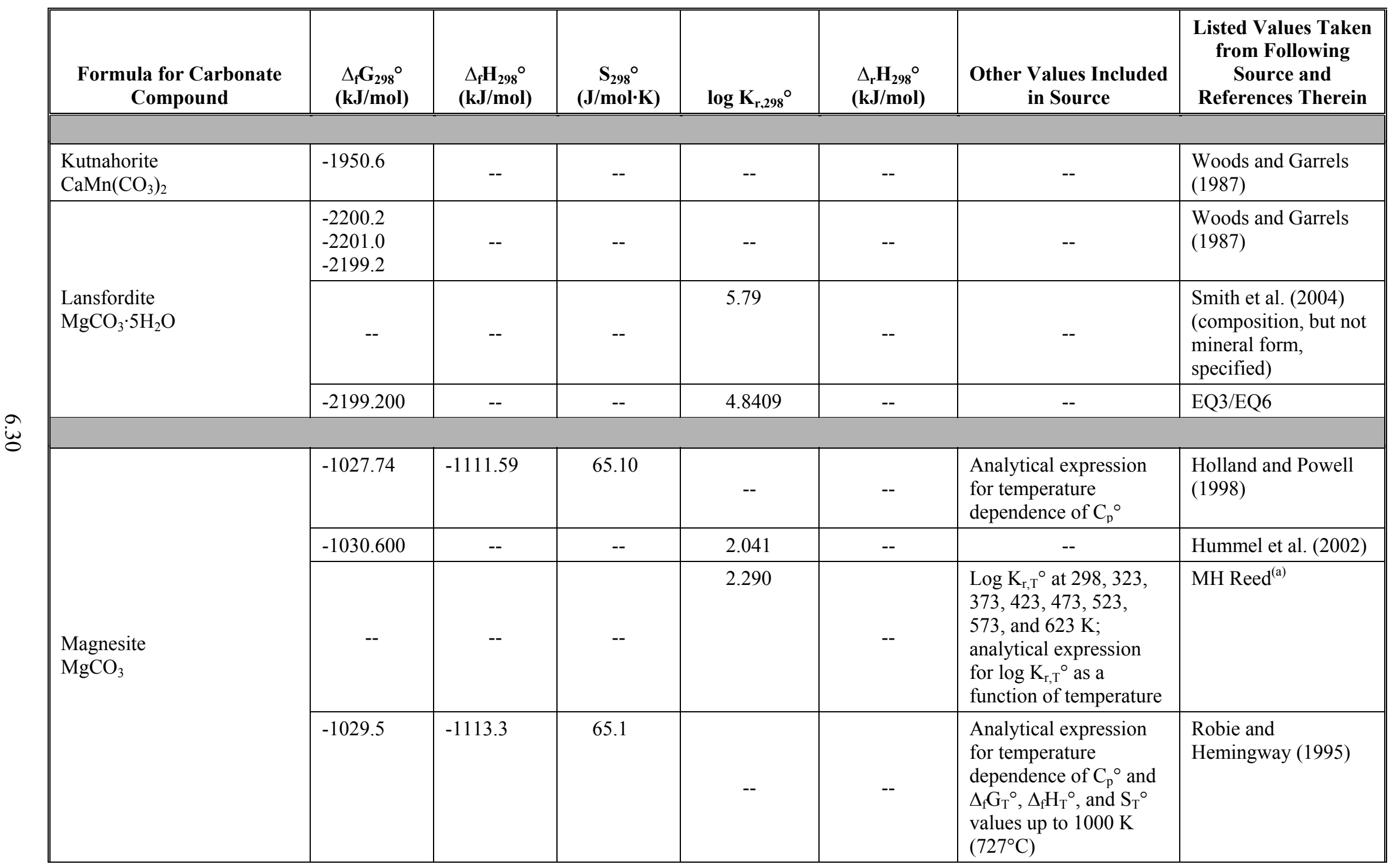


Table 6.4. (contd)

\begin{tabular}{|c|c|c|c|c|c|c|c|}
\hline $\begin{array}{c}\text { Formula for Carbonate } \\
\text { Compound }\end{array}$ & $\begin{array}{c}\Delta_{\mathrm{f}} \mathbf{G}_{298}^{\circ} \\
(\mathbf{k J} / \mathbf{m o l})\end{array}$ & $\begin{array}{c}\Delta_{\mathrm{f}} \mathbf{H}_{298}{ }^{\circ} \\
(\mathbf{k J} / \mathbf{m o l})\end{array}$ & $\begin{array}{c}\mathrm{S}_{298}^{\circ}{ }^{\circ} \\
(\mathrm{J} / \mathrm{mol} \cdot \mathrm{K})\end{array}$ & $\log K_{r, 298}^{\circ}$ & $\begin{array}{c}\Delta_{\mathrm{r}} \mathbf{H}_{298}{ }^{\circ} \\
(\mathbf{k J} / \mathbf{m o l})\end{array}$ & $\begin{array}{l}\text { Other Values Included } \\
\text { in Source }\end{array}$ & $\begin{array}{l}\text { Listed Values Taken } \\
\text { from Following } \\
\text { Source and } \\
\text { References Therein }\end{array}$ \\
\hline \multirow{4}{*}{$\begin{array}{l}\text { Magnesite }^{(\mathrm{c})} \\
\mathrm{MgCO}_{3}\end{array}$} & -- & -- & -- & 2.87 & -- & -- & $\begin{array}{l}\text { Smith et al. (2004) } \\
\text { (only specifies } \\
\text { composition, but not } \\
\text { mineral form) }\end{array}$ \\
\hline & $\begin{array}{l}-1029 \\
-1012.3 \\
-1029.7 \\
-1029.6 \\
-1029.5 \\
-1027.8 \\
-1012.1 \\
-1027.3\end{array}$ & $\begin{array}{l}-1113 \\
-1095.8 \\
-1113.1 \\
-1113.2 \\
-1113.3 \\
-1111.4 \\
-1095.8\end{array}$ & $\begin{array}{l}65.7 \\
65.7 \\
65.7 \\
65.8 \\
65.1 \\
65.7 \\
65.7\end{array}$ & -- & -- & -- & $\begin{array}{l}\text { Woods and Garrels } \\
\text { (1987) }\end{array}$ \\
\hline & -1027.83 & -1111.40 & 65.689 & 2.2936 & -- & $\begin{array}{l}\log \mathrm{K}_{\mathrm{r}, \mathrm{T}}{ }^{\circ} \text { values also at } \\
273,333,373,423, \\
473,523 \text {, and } 573 \mathrm{~K}\end{array}$ & EQ3/EQ6 \\
\hline & -- & -- & -- & 2.8690 & 5.4000 & -- & MINTEQA2 \\
\hline \multirow[b]{2}{*}{$\begin{array}{l}\text { Meionite (Al/Si ordered) } \\
\mathrm{Ca}_{4} \mathrm{Al}_{6} \mathrm{Si}_{6} \mathrm{O}_{24} \mathrm{CO}_{3}\end{array}$} & -13104.74 & -13843.65 & 752.00 & -- & -- & $\begin{array}{l}\text { Analytical expression } \\
\text { for temperature } \\
\text { dependence of } \mathrm{C}_{\mathrm{p}}{ }^{\circ}\end{array}$ & $\begin{array}{l}\text { Holland and Powell } \\
\text { (1998) }\end{array}$ \\
\hline & -- & -- & -- & 77.060 & -- & $\begin{array}{l}\log \mathrm{K}_{\mathrm{r}, \mathrm{T}}{ }^{\circ} \text { at } 298,323, \\
373,423,473,523, \\
573, \text { and } 623 \mathrm{~K} ; \\
\text { analytical expression } \\
\text { for } \log \mathrm{K}_{\mathrm{r}, \mathrm{T}} \mathrm{O}^{\circ} \text { as a } \\
\text { function of temperature }\end{array}$ & MH Reed $^{(\mathrm{a})}$ \\
\hline
\end{tabular}


Table 6.4. (contd)

\begin{tabular}{|c|c|c|c|c|c|c|c|}
\hline $\begin{array}{c}\text { Formula for Carbonate } \\
\text { Compound } \\
\end{array}$ & $\begin{array}{c}\Delta_{\mathrm{f}} \mathbf{G}_{298}{ }^{\circ} \\
(\mathrm{kJ} / \mathbf{m o l}) \\
\end{array}$ & $\begin{array}{c}\Delta_{\mathrm{f}} \mathbf{H}_{298}{ }^{\circ} \\
(\mathbf{k J} / \mathbf{m o l}) \\
\end{array}$ & $\begin{array}{c}\mathrm{S}_{298}^{\circ} \\
(\mathrm{J} / \mathrm{mol} \cdot \mathrm{K}) \\
\end{array}$ & $\log K_{r, 298^{\circ}}$ & $\begin{array}{c}\Delta_{\mathrm{r}} \mathbf{H}_{298}{ }^{\circ} \\
(\mathrm{kJ} / \mathbf{m o l}) \\
\end{array}$ & $\begin{array}{c}\text { Other Values Included } \\
\text { in Source } \\
\end{array}$ & $\begin{array}{c}\text { Listed Values Taken } \\
\text { from Following } \\
\text { Source and } \\
\text { References Therein } \\
\end{array}$ \\
\hline \multirow[t]{2}{*}{$\begin{array}{l}\text { Meionite }(\mathrm{Al} / \mathrm{Si} \text { ordered }) \\
\mathrm{Ca}_{4} \mathrm{Al}_{6} \mathrm{Si}_{6} \mathrm{O}_{24} \mathrm{CO}_{3}\end{array}$} & -13131.8 & -13881.4 & 715.2 & -- & -- & $\begin{array}{l}\text { Analytical expression } \\
\text { for temperature } \\
\text { dependence of } \mathrm{C}_{\mathrm{p}}{ }^{\circ} \text { and } \\
\Delta_{\mathrm{f}} \mathrm{G}_{\mathrm{T}}^{\circ}, \Delta_{\mathrm{f}} \mathrm{H}_{\mathrm{T}}{ }^{\circ}, \text { and } \mathrm{S}_{\mathrm{T}}{ }^{\circ} \\
\text { values up to } 1200 \mathrm{~K} \\
\left(727^{\circ} \mathrm{C}\right)\end{array}$ & $\begin{array}{l}\text { Robie and } \\
\text { Hemingway (1995) }\end{array}$ \\
\hline & -13142.7 & -13897.5 & 691.4 & -- & -- & -- & $\begin{array}{l}\text { Woods and Garrels } \\
\text { (1987) }\end{array}$ \\
\hline & & & & & & & \\
\hline \multirow{4}{*}{$\begin{array}{l}\text { Monohydrocalcite } \\
\mathrm{CaCO}_{3} \cdot \mathrm{H}_{2} \mathrm{O}\end{array}$} & -1361.6 & -1498.3 & 131.1 & -- & -- & -- & $\begin{array}{l}\text { Robie and } \\
\text { Hemingway (1995) }\end{array}$ \\
\hline & -1361.3 & -1498.3 & -- & -- & -- & -- & $\begin{array}{l}\text { Woods and Garrels } \\
(1987)\end{array}$ \\
\hline & -- & -- & -- & 2.73 & -- & -- & Smith et al. (2004) \\
\hline & -1361.600 & -1498.290 & 129.853 & 2.6824 & -- & $\begin{array}{l}\log \mathrm{K}_{\mathrm{r}, \mathrm{T}}{ }^{\circ} \text { values also at } \\
273,333,373,423 \text {, and } \\
473 \mathrm{~K}\end{array}$ & EQ3/EQ6 \\
\hline & & & & & & & \\
\hline \multirow[t]{2}{*}{$\begin{array}{l}\mathrm{Na}_{2} \mathrm{CO}_{3} \\
\text { (likely natrite) }\end{array}$} & -1045.3 & -1129.2 & 135.0 & -- & -- & -- & $\begin{array}{l}\text { Robie and } \\
\text { Hemingway (1995): } \\
\text { (composition, but not } \\
\text { mineral form, } \\
\text { specified) }\end{array}$ \\
\hline & $\begin{array}{l}-1047.7 \\
-1047.8 \\
-1044.4\end{array}$ & $\begin{array}{l}-1130.9 \\
-1131.4 \\
-1130.7\end{array}$ & $\begin{array}{l}136.0 \\
135.0 \\
135.0\end{array}$ & -- & -- & -- & $\begin{array}{l}\text { Woods and Garrels } \\
\text { (1987) }\end{array}$ \\
\hline
\end{tabular}


Table 6.4. (contd)

\begin{tabular}{|c|c|c|c|c|c|c|c|}
\hline $\begin{array}{c}\text { Formula for Carbonate } \\
\text { Compound } \\
\end{array}$ & $\begin{array}{c}\Delta_{\mathrm{f}} \mathbf{G}_{\mathbf{2 9 8}}{ }^{\circ} \\
(\mathrm{kJ} / \mathbf{m o l}) \\
\end{array}$ & $\begin{array}{c}\Delta_{\mathrm{f}} \mathrm{H}_{298}{ }^{\circ} \\
(\mathrm{kJ} / \mathbf{m o l}) \\
\end{array}$ & $\begin{array}{c}\mathrm{S}_{298}{ }^{\circ} \\
(\mathrm{J} / \mathrm{mol} \cdot \mathrm{K}) \\
\end{array}$ & $\log K_{r, 298^{\circ}}$ & $\begin{array}{c}\Delta_{\mathrm{r}} \mathbf{H}_{298}{ }^{\circ} \\
(\mathbf{k J} / \mathbf{m o l}) \\
\end{array}$ & $\begin{array}{l}\text { Other Values Included } \\
\text { in Source }\end{array}$ & $\begin{array}{l}\text { Listed Values Taken } \\
\text { from Following } \\
\text { Source and } \\
\text { References Therein } \\
\end{array}$ \\
\hline $\begin{array}{l}\mathrm{Na}_{2} \mathrm{CO}_{3} \\
\text { (likely natrite) }\end{array}$ & -1046.874 & -1130.680 & 134.980 & 11.1822 & -- & $\begin{array}{l}\log \mathrm{K}_{\mathrm{r}, \mathrm{T}^{\circ} \text { values also at }} \\
273,333,373,423 \text {, } \\
473,523 \text {, and } 573 \mathrm{~K}\end{array}$ & EQ3/EQ6 \\
\hline & & & & & & & \\
\hline \multirow[b]{2}{*}{$\mathrm{Na}_{2} \mathrm{CO}_{3} \cdot 7 \mathrm{H}_{2} \mathrm{O}$} & $\begin{array}{l}-2714.2 \\
-2715.9\end{array}$ & -3200.0 & 422.2 & -- & -- & -- & $\begin{array}{l}\text { Woods and Garrels } \\
\text { (1987) }\end{array}$ \\
\hline & -2714.200 & -3199.189 & 422.200 & 9.9459 & -- & $\begin{array}{l}\log \mathrm{K}_{\mathrm{r}, \mathrm{T}^{\circ} \text { values also at }} \\
273,333,373,423 \text {, and } \\
473 \mathrm{~K}\end{array}$ & EQ3/EQ6 \\
\hline & & & & & & & \\
\hline \multirow{3}{*}{$\begin{array}{l}\text { Nahcolite } \\
\mathrm{NaHCO}_{3}\end{array}$} & -851.2 & -949.0 & 102.1 & -- & -- & -- & $\begin{array}{l}\text { Robie and } \\
\text { Hemingway (1995) }\end{array}$ \\
\hline & $\begin{array}{l}-851.9 \\
-815.9 \\
-851.0 \\
\end{array}$ & $\begin{array}{l}-947.7 \\
-913.4 \\
-950.8 \\
\end{array}$ & $\begin{array}{l}102.1 \\
102.1 \\
101.7 \\
\end{array}$ & -- & -- & -- & $\begin{array}{l}\text { Woods and Garrels } \\
\text { (1987) }\end{array}$ \\
\hline & -849.461 & -947.257 & 102.09 & -0.1118 & -- & $\begin{array}{l}\log \mathrm{K}_{\mathrm{r}, \mathrm{T}}{ }^{\circ} \text { values also at } \\
273,333,373,423 \text {, } \\
473,523 \text {, and } 573 \mathrm{~K}\end{array}$ & EQ3/EQ6 \\
\hline \multirow{3}{*}{$\begin{array}{l}\text { Natron } \\
\mathrm{Na}_{2} \mathrm{CO}_{3} \cdot 10 \mathrm{H}_{2} \mathrm{O}\end{array}$} & $\begin{array}{l}-3431.5 \\
-3427.7 \\
-3429.0\end{array}$ & $\begin{array}{l}-4082.0 \\
-4081.3\end{array}$ & $\begin{array}{l}564.7 \\
562.7\end{array}$ & -- & -- & -- & $\begin{array}{l}\text { Woods and Garrels } \\
\text { (1987) }\end{array}$ \\
\hline & -3427.660 & -4079.394 & 562.700 & 9.6102 & -- & $\begin{array}{l}\log \mathrm{K}_{\mathrm{r}, \mathrm{T}^{\circ}} \text { values also at } \\
273,333,373,423 \text {, } \\
473,523, \text { and } 573 \mathrm{~K}\end{array}$ & EQ3/EQ6 \\
\hline & -- & -- & -- & 9.0180 & 51.2771 & -- & MINTEQA2 \\
\hline
\end{tabular}


Table 6.4. (contd)

\begin{tabular}{|c|c|c|c|c|c|c|c|}
\hline $\begin{array}{c}\text { Formula for Carbonate } \\
\text { Compound }\end{array}$ & $\begin{array}{c}\Delta_{\mathrm{f}} \mathbf{G}_{298}^{\circ} \\
(\mathbf{k J} / \mathbf{m o l})\end{array}$ & $\begin{array}{c}\Delta_{\mathrm{f}} \mathbf{H}_{298}{ }^{\circ} \\
(\mathrm{kJ} / \mathbf{m o l})\end{array}$ & $\begin{array}{c}\mathrm{S}_{298}^{\circ} \\
(\mathrm{J} / \mathbf{m o l} \cdot \mathbf{K})\end{array}$ & $\log K_{r, 298^{\circ}}$ & $\begin{array}{c}\Delta_{\mathrm{r}} \mathbf{H}_{298}^{\circ} \\
(\mathbf{k J} / \mathbf{m o l})\end{array}$ & $\begin{array}{l}\text { Other Values Included } \\
\text { in Source }\end{array}$ & $\begin{array}{l}\text { Listed Values Taken } \\
\text { from Following } \\
\text { Source and } \\
\text { References Therein }\end{array}$ \\
\hline \multirow{6}{*}{$\begin{array}{l}\text { Nesquehonite } \\
\mathrm{MgCO}_{3} \cdot 3 \mathrm{H}_{2} \mathrm{O}\end{array}$} & -- & -- & -- & 4.996 & -- & $\begin{array}{l}\log \mathrm{K}_{\mathrm{r}, \mathrm{T}}{ }^{\circ} \text { at } 298,323, \\
373,423,473,523, \\
573, \text { and } 623 \mathrm{~K} ; \\
\text { analytical expression } \\
\text { for } \log \mathrm{K}_{\mathrm{r}, \mathrm{T}}{ }^{\circ} \text { as a } \\
\text { function of temperature }\end{array}$ & "MH Reed ${ }^{(\text {a) }}$ \\
\hline & -1723.8 & -1977.3 & 195.6 & -- & -- & -- & $\begin{array}{l}\text { Robie and } \\
\text { Hemingway (1995) }\end{array}$ \\
\hline & -- & -- & -- & 5.66 & -- & -- & $\begin{array}{l}\text { Smith et al. (2004) } \\
\text { (composition, but not } \\
\text { mineral form, } \\
\text { specified) }\end{array}$ \\
\hline & $\begin{array}{l}-1726.6 \\
-1726.6 \\
-1723.7 \\
-1724.0 \\
-1726.1 \\
\end{array}$ & $\begin{array}{l}-1985.7 \\
-1977.3 \\
-1977.2\end{array}$ & $\begin{array}{l}159.0 \\
195.6 \\
195.6\end{array}$ & -- & -- & -- & $\begin{array}{l}\text { Woods and Garrels } \\
\text { (1987) }\end{array}$ \\
\hline & -1723.95 & -1977.26 & 195.64 & 4.9955 & -- & $\begin{array}{l}\log \mathrm{K}_{\mathrm{r}, \mathrm{T}}{ }^{\circ} \text { values also at } \\
273,333,373,423 \text {, } \\
473,523 \text {, and } 573 \mathrm{~K}\end{array}$ & EQ3/EQ6 \\
\hline & -- & -- & -- & 5.6590 & -38.8312 & -- & MINTEQA2 \\
\hline $\begin{array}{l}\text { Pirssonite } \\
\mathrm{Na}_{2} \mathrm{Ca}\left(\mathrm{CO}_{3}\right)_{2} \cdot 2 \mathrm{H}_{2} \mathrm{O}\end{array}$ & -635.794 & -- & -- & 11.3230 & -- & -- & EQ3/EQ6 \\
\hline
\end{tabular}


Table 6.4. (contd)

\begin{tabular}{|c|c|c|c|c|c|c|c|}
\hline $\begin{array}{c}\text { Formula for Carbonate } \\
\text { Compound }\end{array}$ & $\begin{array}{c}\Delta_{\mathrm{f}} \mathbf{G}_{298}^{\circ} \\
(\mathbf{k J} / \mathbf{m o l})\end{array}$ & $\begin{array}{c}\Delta_{\mathrm{f}} \mathbf{H}_{298}^{\circ} \\
(\mathbf{k J} / \mathbf{m o l})\end{array}$ & $\begin{array}{c}\mathrm{S}_{298}^{\circ}{ }^{\circ} \\
(\mathrm{J} / \mathbf{m o l} \cdot \mathbf{K})\end{array}$ & $\log K_{r, 298^{\circ}}$ & $\begin{array}{c}\Delta_{\mathrm{r}} \mathrm{H}_{298}^{\circ} \\
(\mathbf{k J} / \mathbf{m o l})\end{array}$ & $\begin{array}{l}\text { Other Values Included } \\
\text { in Source }\end{array}$ & $\begin{array}{l}\text { Listed Values Taken } \\
\text { from Following } \\
\text { Source and } \\
\text { References Therein }\end{array}$ \\
\hline \multirow{6}{*}{$\begin{array}{l}\text { Rhodochrosite } \\
\mathrm{MnCO}_{3}\end{array}$} & -817.22 & -891.06 & 98.00 & -- & -- & $\begin{array}{l}\text { analytical expression } \\
\text { for temperature } \\
\text { dependence of } \mathrm{C}_{\mathrm{p}}{ }^{\circ}\end{array}$ & $\begin{array}{l}\text { Holland and Powell } \\
\text { (1998) }\end{array}$ \\
\hline & -819.548 & -890.081 & -- & -0.801 & -20.884 & -- & Hummel et al. (2002) \\
\hline & -- & -- & -- & -0.80 & -20.88 & -- & $\begin{array}{l}\text { Nordstrom et al. } \\
\text { (1990) }\end{array}$ \\
\hline & -- & -- & -- & 0.026 & -- & $\begin{array}{l}\log \mathrm{K}_{\mathrm{r}, \mathrm{T}}{ }^{\circ} \text { at } 298,323, \\
373,423,473,523, \\
573, \text { and } 623 \mathrm{~K} ; \\
\text { analytical expression } \\
\text { for } \log \mathrm{K}_{\mathrm{r}, \mathrm{T}} \mathrm{O}^{\circ} \text { as a } \\
\text { function of temperature }\end{array}$ & M. H. Reed ${ }^{(a)}$ \\
\hline & -819.1 & -892.9 & 98.0 & -- & -- & $\begin{array}{l}\text { analytical expression } \\
\text { for temperature } \\
\text { dependence of } \mathrm{C}_{\mathrm{p}}^{\circ} \text { and } \\
\Delta_{\mathrm{f}} \mathrm{G}_{\mathrm{T}}^{\circ}, \Delta_{\mathrm{f}} \mathrm{H}_{\mathrm{T}}{ }^{\circ} \text {, and } \mathrm{S}_{\mathrm{T}}{ }^{\circ} \\
\text { values up to } 600 \mathrm{~K} \\
\left(327^{\circ} \mathrm{C}\right)\end{array}$ & $\begin{array}{l}\text { Robie and } \\
\text { Hemingway (1995) }\end{array}$ \\
\hline & $\begin{array}{l}-816.7 \\
-815.5 \\
-816.0 \\
-816.1 \\
-816.7 \\
-815.0 \\
-818.8\end{array}$ & $\begin{array}{l}-894.1 \\
-888.6 \\
-889.3 \\
-889.2 \\
-894.1 \\
-889.3\end{array}$ & $\begin{array}{r}85.8 \\
100.0 \\
100.0 \\
85.8\end{array}$ & -- & -- & -- & $\begin{array}{l}\text { Woods and Garrels } \\
\text { (1987) } \\
\text { (natural sample) }\end{array}$ \\
\hline
\end{tabular}


Table 6.4. (contd)

\begin{tabular}{|c|c|c|c|c|c|c|c|}
\hline $\begin{array}{c}\text { Formula for Carbonate } \\
\text { Compound }\end{array}$ & $\begin{array}{c}\Delta_{\mathrm{f}} \mathbf{G}_{298}{ }^{\circ} \\
(\mathbf{k J} / \mathbf{m o l})\end{array}$ & $\begin{array}{c}\Delta_{\mathrm{f}} \mathrm{H}_{298}{ }^{\circ} \\
(\mathrm{kJ} / \mathbf{m o l})\end{array}$ & $\begin{array}{c}\mathrm{S}_{298}^{\circ} \\
(\mathrm{J} / \mathrm{mol} \cdot \mathrm{K}) \\
\end{array}$ & $\log K_{r, 298^{\circ}}$ & $\begin{array}{c}\Delta_{\mathrm{r}} \mathbf{H}_{298}{ }^{\circ} \\
(\mathrm{kJ} / \mathbf{m o l})\end{array}$ & $\begin{array}{l}\text { Other Values Included } \\
\text { in Source }\end{array}$ & $\begin{array}{l}\text { Listed Values Taken } \\
\text { from Following } \\
\text { Source and } \\
\text { References Therein }\end{array}$ \\
\hline \multirow{4}{*}{$\begin{array}{l}\text { Rhodochrosite } \\
\mathrm{MnCO}_{3}\end{array}$} & -- & -- & -- & -0.67 & -- & $\begin{array}{l}\log \mathrm{K}_{\mathrm{r}, \mathrm{T}} \text { listed at one or } \\
\text { more values of ionic } \\
\text { strength }\end{array}$ & Smith et al. (2004) \\
\hline & -816.068 & -889.188 & 99.998 & -0.1928 & -- & $\begin{array}{l}\log \mathrm{K}_{\mathrm{r}, \mathrm{T}}{ }^{\circ} \text { values also at } \\
273,333,373,423, \\
473,523, \text { and } 573 \mathrm{~K}\end{array}$ & EQ3/EQ6 \\
\hline & -- & -- & -- & -0.2510 & -16.4800 & -- & MINTEQA2 \\
\hline & -- & -- & -- & -0.801 & -20.883 & -- & PHREEQC \\
\hline & & & & & & & \\
\hline \multirow{4}{*}{$\begin{array}{l}\text { Rhodochrosite (synthetic) } \\
\mathrm{MnCO}_{3}\end{array}$} & -815.324 & -896.064 & -- & -0.061 & -14.901 & -- & Hummel et al. (2002) \\
\hline & -- & -- & -- & -0.061 & -- & -- & $\begin{array}{l}\text { Nordstrom et al. } \\
(1990)\end{array}$ \\
\hline & -817.6 & -895.0 & 85.6 & -- & -- & -- & $\begin{array}{l}\text { Woods and Garrels } \\
\text { (1987) (form not } \\
\text { specified) }\end{array}$ \\
\hline & -- & -- & -- & -0.17 & -- & $\begin{array}{l}\log \mathrm{K}_{\mathrm{r}, \mathrm{T}} \text { listed at one or } \\
\text { more values of ionic } \\
\text { strength }\end{array}$ & $\begin{array}{l}\text { Smith et al. (2004) } \\
\text { (only specifies } \\
\text { composition, but not } \\
\text { mineral form) }\end{array}$ \\
\hline $\begin{array}{l}\text { Rhodochrosite (precipitated) } \\
\mathrm{MnCO}_{3}\end{array}$ & -813.0 & $\begin{array}{l}-887 \\
-883.2\end{array}$ & 99.6 & -- & -- & -- & $\begin{array}{l}\text { Woods and Garrels } \\
\text { (1987) (labeled as } \\
\text { precipitated form) }\end{array}$ \\
\hline
\end{tabular}


Table 6.4. (contd)

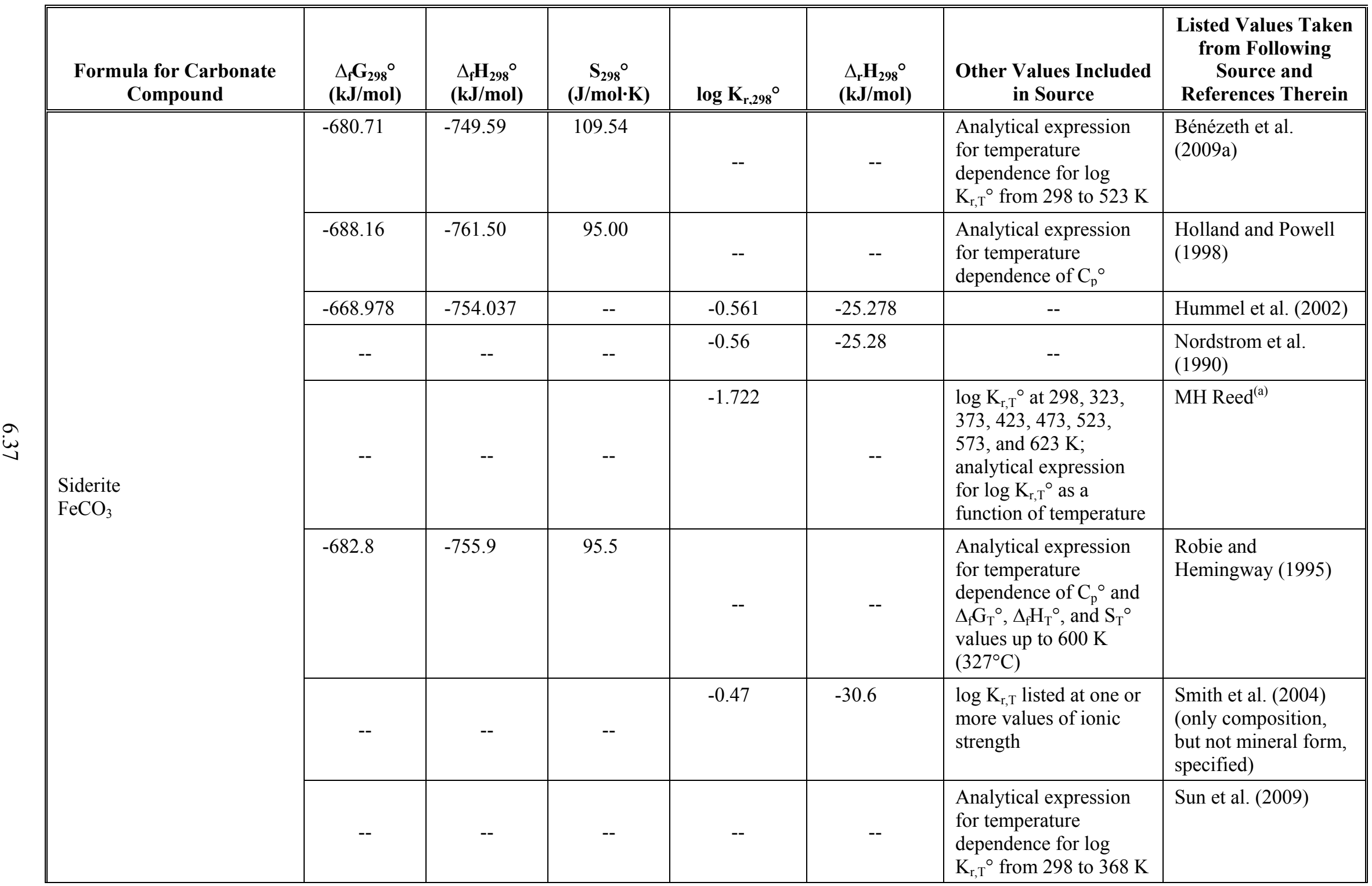


Table 6.4. (contd)

\begin{tabular}{|c|c|c|c|c|c|c|c|}
\hline $\begin{array}{c}\text { Formula for Carbonate } \\
\text { Compound }\end{array}$ & $\begin{array}{c}\Delta_{\mathbf{f}} \mathbf{G}_{298}{ }^{\circ} \\
(\mathbf{k J} / \mathbf{m o l})\end{array}$ & $\begin{array}{c}\Delta_{\mathrm{f}} \mathbf{H}_{298}{ }^{\circ} \\
(\mathbf{k J} / \mathbf{m o l}) \\
\end{array}$ & $\begin{array}{c}\mathrm{S}_{298}^{\circ} \\
(\mathrm{J} / \mathrm{mol} \cdot \mathrm{K}) \\
\end{array}$ & $\log K_{r, 298^{\circ}}$ & $\begin{array}{c}\Delta_{\mathrm{r}} \mathbf{H}_{298}{ }^{\circ} \\
(\mathbf{k J} / \mathbf{m o l}) \\
\end{array}$ & $\begin{array}{c}\text { Other Values Included } \\
\text { in Source }\end{array}$ & $\begin{array}{c}\text { Listed Values Taken } \\
\text { from Following } \\
\text { Source and } \\
\text { References Therein }\end{array}$ \\
\hline \multirow{4}{*}{$\begin{array}{l}\text { Siderite } \\
\mathrm{FeCO}_{3}\end{array}$} & $\begin{array}{l}-673.9 \\
-680.3 \\
-679.5\end{array}$ & $\begin{array}{l}-747.7 \\
-753.1 \\
-752.2\end{array}$ & $\begin{array}{l}92.9 \\
96.1 \\
96.1\end{array}$ & -- & -- & -- & $\begin{array}{l}\text { Woods and Garrels } \\
\text { (1987) }\end{array}$ \\
\hline & -679.540 & -749.660 & 105.02 & -0.1920 & -- & $\begin{array}{l}\log \mathrm{K}_{\mathrm{r}, \mathrm{T}}{ }^{\circ} \text { values also at } \\
273,333,373,423 \text {, } \\
473,523 \text {, and } 573 \mathrm{~K}\end{array}$ & EQ3/EQ6 \\
\hline & -- & -- & -- & 0.0890 & -30.6000 & -- & MINTEQA2 \\
\hline & -- & -- & -- & -0.561 & -25.280 & -- & PHREEQC \\
\hline & & & & & & & \\
\hline \multirow[b]{2}{*}{$\mathrm{FeCO}_{3}$ (precipitated form) } & -666.467 & -764.414 & -- & -0.121 & -14.901 & -- & Hummel et al. (2002) \\
\hline & -- & -- & -- & -0.12 & -- & -- & $\begin{array}{l}\text { Nordstrom et al. } \\
(1990)\end{array}$ \\
\hline & & & & & & & \\
\hline \multirow{3}{*}{$\begin{array}{l}\text { Spurrite } \\
\mathrm{Ca}_{5} \mathrm{Si}_{2} \mathrm{O}_{8} \mathrm{CO}_{3}\end{array}$} & -5534.67 & -5849.69 & 330.00 & -- & -- & $\begin{array}{l}\text { Analytical expression } \\
\text { for temperature } \\
\text { dependence of } \mathrm{C}_{\mathrm{p}}{ }^{\circ}\end{array}$ & $\begin{array}{l}\text { Holland and Powell } \\
\text { (1998) }\end{array}$ \\
\hline & -- & -- & -- & 75.201 & -- & $\begin{array}{l}\log \mathrm{K}_{\mathrm{r}, \mathrm{T}}{ }^{\circ} \text { at } 298,323, \\
373,423,473,523, \\
573, \text { and } 623 \mathrm{~K} ; \\
\text { analytical expression } \\
\text { for } \log \mathrm{K}_{\mathrm{r}, \mathrm{T}}{ }^{\circ} \text { as a } \\
\text { function of temperature }\end{array}$ & MH Reed ${ }^{(a)}$ \\
\hline & -5525.6 & -5840.2 & 331.0 & -- & -- & $\begin{array}{l}\text { Analytical expression } \\
\text { for temperature } \\
\text { dependence of } \mathrm{C}_{\mathrm{p}}{ }^{\circ} \text { and } \\
\Delta_{\mathrm{f}} \mathrm{G}_{\mathrm{T}}^{\circ}, \Delta_{\mathrm{f}} \mathrm{H}_{\mathrm{T}}{ }^{\circ}, \text { and } \mathrm{S}_{\mathrm{T}}{ }^{\circ} \\
\text { values up to } 1300 \mathrm{~K} \\
\left(1027^{\circ} \mathrm{C}\right)\end{array}$ & $\begin{array}{l}\text { Robie and } \\
\text { Hemingway (1995) }\end{array}$ \\
\hline
\end{tabular}


Table 6.4. (contd)

\begin{tabular}{|c|c|c|c|c|c|c|c|}
\hline $\begin{array}{c}\text { Formula for Carbonate } \\
\text { Compound }\end{array}$ & $\begin{array}{c}\Delta_{\mathrm{f}} \mathbf{G}_{298}{ }^{\circ} \\
(\mathbf{k J} / \mathbf{m o l}) \\
\end{array}$ & $\begin{array}{c}\Delta_{\mathrm{f}} \mathbf{H}_{298}{ }^{\circ} \\
(\mathbf{k J} / \mathbf{m o l}) \\
\end{array}$ & $\begin{array}{c}\mathrm{S}_{298}{ }^{\circ} \\
(\mathrm{J} / \mathrm{mol} \cdot \mathrm{K}) \\
\end{array}$ & $\log K_{r, 298^{\circ}}$ & $\begin{array}{c}\Delta_{\mathrm{r}} \mathbf{H}_{298}{ }^{\circ} \\
(\mathbf{k J} / \mathbf{m o l})\end{array}$ & $\begin{array}{l}\text { Other Values Included } \\
\text { in Source }\end{array}$ & $\begin{array}{l}\text { Listed Values Taken } \\
\text { from Following } \\
\text { Source and } \\
\text { References Therein }\end{array}$ \\
\hline $\begin{array}{l}\text { Spurrite } \\
\mathrm{Ca}_{5} \mathrm{Si}_{2} \mathrm{O}_{8} \mathrm{CO}_{3}\end{array}$ & -5568.8 & -5899.3 & 270.8 & -- & -- & -- & $\begin{array}{l}\text { Woods and Garrels } \\
\text { (1987) }\end{array}$ \\
\hline \multirow{4}{*}{$\begin{array}{l}\text { Thermonatrite } \\
\mathrm{Na}_{2} \mathrm{CO}_{3} \cdot \mathrm{H}_{2} \mathrm{O}\end{array}$} & -1286.1 & -1429.7 & 168.1 & -- & -- & $\begin{array}{l}\text { Analytical expression } \\
\text { for temperature } \\
\text { dependence of } \mathrm{C}_{\mathrm{p}}^{\circ} \text { up to } \\
380 \mathrm{~K}\left(107^{\circ} \mathrm{C}\right)\end{array}$ & $\begin{array}{l}\text { Robie and } \\
\text { Hemingway (1995) }\end{array}$ \\
\hline & $\begin{array}{l}-1288.7 \\
-1285.3 \\
-1286.0 \\
-1286.6\end{array}$ & $\begin{array}{l}-1432.0 \\
-1431.3\end{array}$ & $\begin{array}{l}168.2 \\
168.1\end{array}$ & -- & -- & -- & $\begin{array}{l}\text { Woods and Garrels } \\
\text { (1987) }\end{array}$ \\
\hline & -1285.310 & -1428.784 & 168.110 & 10.9623 & -- & $\begin{array}{l}\log \mathrm{K}_{\mathrm{r}, \mathrm{T}}{ }^{\circ} \text { values also at } \\
273,333,373,423 \text {, } \\
473,523 \text {, and } 573 \mathrm{~K}\end{array}$ & EQ3/EQ6 \\
\hline & -- & -- & -- & 10.9660 & -25.0799 & -- & MINTEQA2 \\
\hline & & & & & & & \\
\hline \multirow{3}{*}{$\begin{array}{l}\text { Tilleyite } \\
\mathrm{Ca}_{5} \mathrm{Si}_{2} \mathrm{O}_{7}\left(\mathrm{CO}_{3}\right)_{2}\end{array}$} & -6008.28 & -6368.33 & 390.00 & -- & -- & $\begin{array}{l}\text { Analytical expression } \\
\text { for temperature } \\
\text { dependence of } C_{p}^{\circ}\end{array}$ & $\begin{array}{l}\text { Holland and Powell } \\
\text { (1998) }\end{array}$ \\
\hline & -- & -- & -- & 53.490 & -- & $\begin{array}{l}\log \mathrm{K}_{\mathrm{r}, \mathrm{T}}{ }^{\circ} \text { at } 298,323, \\
373,423,473,523, \\
573, \text { and } 623 \mathrm{~K} ; \\
\text { analytical expression } \\
\text { for } \log \mathrm{K}_{\mathrm{r}, \mathrm{T}} \mathrm{O}^{\circ} \text { as a } \\
\text { function of temperature }\end{array}$ & MH Reed ${ }^{(a)}$ \\
\hline & -6013.5 & -6372.2 & 394.0 & -- & -- & $\begin{array}{l}\text { Analytical expression } \\
\text { for temperature } \\
\text { dependence of } \mathrm{C}_{\mathrm{p}}{ }^{\circ} \text { and } \\
\Delta_{\mathrm{f}} \mathrm{G}_{\mathrm{T}}^{\circ}, \Delta_{\mathrm{f}} \mathrm{H}_{\mathrm{T}}{ }^{\circ}, \text { and } \mathrm{S}_{\mathrm{T}}{ }^{\circ} \\
\text { values up to } 1200 \mathrm{~K} \\
\left(927^{\circ} \mathrm{C}\right)\end{array}$ & $\begin{array}{l}\text { Robie and } \\
\text { Hemingway (1995) }\end{array}$ \\
\hline
\end{tabular}


Table 6.4. (contd)

\begin{tabular}{|c|c|c|c|c|c|c|c|}
\hline $\begin{array}{c}\text { Formula for Carbonate } \\
\text { Compound }\end{array}$ & $\begin{array}{c}\Delta_{\mathbf{f}} \mathbf{G}_{298}{ }^{\circ} \\
(\mathbf{k J} / \mathbf{m o l})\end{array}$ & $\begin{array}{c}\Delta_{\mathbf{f}} \mathbf{H}_{298}{ }^{\circ} \\
(\mathbf{k J} / \mathbf{m o l})\end{array}$ & $\begin{array}{c}\mathrm{S}_{298}^{\circ} \\
(\mathrm{J} / \mathrm{mol} \cdot \mathrm{K})\end{array}$ & $\log K_{r, 298^{\circ}}$ & $\begin{array}{c}\Delta_{\mathrm{r}} \mathbf{H}_{298}{ }^{\circ} \\
(\mathbf{k J} / \mathbf{m o l})\end{array}$ & $\begin{array}{c}\text { Other Values Included } \\
\text { in Source }\end{array}$ & $\begin{array}{l}\text { Listed Values Taken } \\
\text { from Following } \\
\text { Source and } \\
\text { References Therein }\end{array}$ \\
\hline $\begin{array}{l}\text { Tilleyite } \\
\mathrm{Ca}_{5} \mathrm{Si}_{2} \mathrm{O}_{7}\left(\mathrm{CO}_{3}\right)_{2}\end{array}$ & -6009.8 & -6371.6 & 376.7 & -- & -- & -- & $\begin{array}{l}\text { Woods and Garrels } \\
\text { (1987) }\end{array}$ \\
\hline & & & & & & & \\
\hline \multirow{2}{*}{$\begin{array}{l}\text { Trona } \\
\mathrm{Na}_{3}\left(\mathrm{CO}_{3}\right)\left(\mathrm{HCO}_{3}\right) \cdot 2 \mathrm{H}_{2} \mathrm{O}\end{array}$} & -- & -2682.1 & -- & -- & -- & -- & $\begin{array}{l}\text { Robie and } \\
\text { Hemingway (1995) }\end{array}$ \\
\hline & $\begin{array}{l}-2386.6 \\
-2383.4 \\
-2380.5 \\
\end{array}$ & -2684.9 & 301.2 & -- & -- & -- & $\begin{array}{l}\text { Woods and Garrels } \\
(1987)\end{array}$ \\
\hline \multirow[t]{2}{*}{$\begin{array}{l}\text { Trona-K } \\
\mathrm{K}_{2} \mathrm{NaH}\left(\mathrm{CO}_{3}\right)_{2} \cdot 2 \mathrm{H}_{2} \mathrm{O}\end{array}$} & -575.740 & -- & -- & 11.5891 & -- & - & EQ3/EQ6 \\
\hline & & & & & & & \\
\hline \multirow{3}{*}{$\begin{array}{l}\text { Vaterite } \\
\mathrm{CaCO}_{3}\end{array}$} & -1125.5 & -- & -- & -- & -- & -- & $\begin{array}{l}\text { Robie and } \\
\text { Hemingway (1995) }\end{array}$ \\
\hline & -1125.5 & -- & -- & -- & -- & -- & $\begin{array}{l}\text { Woods and Garrels } \\
\text { (1987) }\end{array}$ \\
\hline & -- & -- & -- & 2.42 & -29.6 & -- & Smith et al. (2004) \\
\hline $\begin{array}{l}\text { Wegscheiderite } \\
\mathrm{Na}_{5}\left(\mathrm{HCO}_{3}\right)_{3} \mathrm{CO}_{3}\end{array}$ & -- & -3984.0 & -- & -- & -- & -- & $\begin{array}{l}\text { Robie and } \\
\text { Hemingway (1995) }\end{array}$ \\
\hline \multicolumn{8}{|c|}{$\begin{array}{l}\text { (a) Reed MH. 2008. "SOLTHERM Database of Equilibria Constants." Personal communication, University of Oregon, Eugene, January } 31,2008 . \\
\text { (b) Bénézeth et al. (2009b) have determined the solubility product as a function of temperature for a natural sample of dolomite based on solubility } \\
\text { measurements from } 25^{\circ} \mathrm{C} \text { to } 200^{\circ} \mathrm{C} \text {. Their calculated solubility product values are not listed in their extended abstract. } \\
\text { (c) Bénézeth et al. }(2009 \mathrm{~b}) \text { have determined the solubility product as a function of temperature for synthesized magnesite based on solubility measurements } \\
\text { from } 25^{\circ} \mathrm{C} \text { to } 200^{\circ} \mathrm{C} \text {. Their calculated solubility product values are not listed in their extended abstract. } \\
\text { (d) Munemoto and Fukushi }(2009) \text { have determined the solubility product for synthesized monohydrocalcite based on solubility measurements from } 5^{\circ} \mathrm{C} \text { to } \\
40^{\circ} \mathrm{C} \text {. Their calculated value for the solubility product of monohydrocalcite is not listed in their extended abstract. }\end{array}$} \\
\hline
\end{tabular}




\subsection{Comparison of Carbonate Characterization Data to Known Thermodynamic Data}

Table 7.1 lists all the carbonate minerals given in Tables 5.2, 5.3, and 5.4, which were physically identified as being present in the host rock formations at $\mathrm{CO}_{2}$ injection test sites or natural analogue sites, reaction products of $\mathrm{CO}_{2}$ injection/intrusion into a host formation, or as mineral reactants or products of laboratory $\mathrm{CO}_{2}$ fluid-rock/-mineral experiments. Almost all of the carbonates in Table 7.1 were identified in studies of $\mathrm{CO}_{2}$ natural analogue sites or laboratory $\mathrm{CO}_{2}$ fluid-rock/-mineral experiments. The carbonate mineralogy obviously must be known for all host formations at sites that are being used or considered for $\mathrm{CO}_{2}$ injection testing. This mineralogical information was not detailed in the publications (Table 5.2) reviewed for our evaluation but was probably given in references cited by these publications. However, many of the publications listed in Table 5.2 used geochemical reaction modeling to explain $\mathrm{CO}_{2}$ gas-fluid-rock geochemistry at their sites.

The list of carbonate minerals in Table 7.1 is short and contains typically common carbonate minerals. Except for ferroan and magnesian calcites and ferroan dolomite, data (Table 6.4) are available for the key thermodynamic parameters of the carbonate minerals in Table 7.1. With respect to availability of thermodynamic data, this is good information for those using geochemical reaction modeling techniques to interpret information and results from $\mathrm{CO}_{2}$ injection and natural analogue sites or laboratory experiments. However, as stated previously, Railsback (1999) noted that in the future, more thorough mineralogical characterization of geological systems affected by anthropogenic activities might show greater diversity of carbonate mineralogy than traditional assumptions would have previously suggested. 
Table 7.1. Carbonate Minerals Physically Identified in Host Rock Formations at $\mathrm{CO}_{2}$ Injection or Natural Analogue Sites, Reaction Products of $\mathrm{CO}_{2}$ Injection/Intrusion into a Host Formation, or as Mineral Reactants or Products of Laboratory $\mathrm{CO}_{2}$ Fluid-Rock/-Mineral Experiments

\begin{tabular}{|c|c|}
\hline $\begin{array}{c}\text { Carbonate Minerals Physically } \\
\text { Identified as Being in the Geologic } \\
\text { Formation, Products of } \mathrm{CO}_{2} \\
\text { Injection/Intrusion into a Host } \\
\text { Formation, or as Reactants or Products } \\
\text { of Laboratory Experiments } \\
\end{array}$ & Reference and Type of Study \\
\hline Ankerite $^{(a)}$ & $\begin{array}{l}\text { - } \\
\text { - } \text { Bertier et al. (2006) - laboratory fluid-rock experiments } \\
\text { - } \text { McGrail et al. (2006) - experimental fluid-rock experiments } \\
\text { - Pauwels et al. (2007) - natural analogue } \\
\text { - } \quad \text { Watson et al. (2004) - natural analogue }\end{array}$ \\
\hline Aragonite & $\begin{array}{l}\text { - Bertier et al. (2006) - laboratory fluid-rock experiments } \\
\text { - Jacquemet et al. (2008) - laboratory fluid-rock (cement) } \\
\text { experiments }\end{array}$ \\
\hline Calcite & $\begin{array}{l}\text { - Bateman et al. (2005) - laboratory fluid-rock experiments } \\
\text { - Bertier et al. (2006) - laboratory fluid-rock experiments } \\
\text { - Guichet et al. (2005) (abstract only) - laboratory fluid-rock } \\
\text { - } \text { experiments } \\
\text { - } \text { experiments } \\
\text { - Lin et al. (2008) - laboratory fluid-rock and fluid-mineral } \\
\text { - } \text { - Macheriments } \\
\text { - May (2005) - field injection site } \\
\text { - McGrail et al. (2006) - experimental fluid-rock experiments } \\
\text { - Michael and Buschkuehle (2006) - natural analogue } \\
\text { - Pearce et al. (1996) - natural analogue (review of information) } \\
\text { - } \text { and experimental fluid-rock experiments } \\
\text { - Vosenbauer et al. (2005) - laboratory fluid-rock experiments } \\
\text { - Vosteen and May (2007) (abstract only) - natural analogue }\end{array}$ \\
\hline Calcite, ferroan & - Worden (2006) - natural analogue \\
\hline Calcite, magnesian & - Park and Fan (2004) - laboratory fluid-rock experiments \\
\hline $\begin{array}{l}\text { Carbonate, ferroan } \\
\text { (specific carbonate mineral not identified) }\end{array}$ & - Watson et al. (2004) - natural analogue \\
\hline $\begin{array}{l}\text { Carbonate, magnesian } \\
\text { (specific carbonate mineral not identified) }\end{array}$ & - Watson et al. (2004) - natural analogue \\
\hline Dawsonite & $\begin{array}{l}\text { - Moore et al. (2005) - natural analogue } \\
\text { - Palandri et al. (2005) - experimental fluid-mineral experiments } \\
\text { - Worden (2006) - natural analogue }\end{array}$ \\
\hline
\end{tabular}


Table 7.1. (contd)

\begin{tabular}{|c|c|}
\hline $\begin{array}{l}\text { Carbonate Minerals Physically } \\
\text { Identified as Being in the Geologic } \\
\text { Formation, Products of } \mathrm{CO}_{2} \\
\text { Injection/Intrusion into a Host } \\
\text { Formation, or as Reactants or Products } \\
\text { of Laboratory Experiments }\end{array}$ & Reference and Type of Study \\
\hline Dolomite & $\begin{array}{l}\text { - Bateman et al. (2005) - laboratory fluid-rock experiments } \\
\text { - Bertier et al. (2006) - laboratory fluid-rock experiments } \\
\text { - Machel (2005) - field injection site } \\
\text { - May (2005) - natural analogue } \\
\text { - Moore et al. (2005) - natural analogue } \\
\text { - Pearce et al. (1996) - natural analogue (review of information) } \\
\text { and experimental fluid-rock experiments } \\
\text { - Rosenbauer et al. (2005) - laboratory fluid-rock experiments } \\
\text { - Shiraki and Dunn (2000) - laboratory fluid-rock experiments } \\
\text { - Vosteen and May (2007) (abstract only) - natural analogue }\end{array}$ \\
\hline Dolomite, ferroan & $\begin{array}{l}\text { - Pauwels et al. (2007) - natural analogue } \\
\text { - Pearce et al. (1996) - natural analogue (review of information) } \\
\text { and experimental fluid-rock experiments } \\
\text { - Worden (2006) - natural analogue }\end{array}$ \\
\hline Magnesite & $\begin{array}{l}\text { - Kaszuba et al. (2003) - laboratory fluid-rock experiments } \\
\text { - Kaszuba et al. (2005) - laboratory fluid-rock experiments } \\
\text { - Schulze et al. (2004) - laboratory fluid-rock experiments } \\
\text { - Wolf et al. (2004) - laboratory fluid-rock experiments }\end{array}$ \\
\hline Siderite & $\begin{array}{l}\text { - Kaszuba et al. (2003) - laboratory fluid-rock experiments } \\
\text { - Kaszuba et al. (2005) - laboratory fluid-rock experiments } \\
\text { - Schulze et al. (2004) - laboratory fluid-rock experiments } \\
\text { - Wolf et al. (2004) - laboratory fluid-rock experiments }\end{array}$ \\
\hline \multicolumn{2}{|c|}{$\begin{array}{l}\text { (a) Pure } \mathrm{Fe} \text { end member ankerite }\left[\mathrm{Ca}\left(\mathrm{Fe}\left(\mathrm{CO}_{3}\right)_{2}\right] \text { has not been found in nature or produced in the laboratory }\right. \\
\text { (Reeder 1983a). }\end{array}$} \\
\hline
\end{tabular}




\subsection{Carbonate Mineral Solid Solutions}

Solid solutions (crystal solutions) of different carbonate minerals are common. Carbonate solid solutions are important to understanding the phase relations of carbonates in geochemical systems and thus are important to accurate geochemical reaction modeling of $\mathrm{CO}_{2}$ sequestration systems and mineral trapping reactions. Theoretical concepts and methods of solid solution thermodynamics needed for modeling aqueous-solid solution geochemical systems are presented in detail by Bruno et al. (2007), Glynn (1990, 2000), Navrotsky (1987), Nordstrom and Munoz (1985), and others, and will therefore not be repeated here. Extensive experimental studies have been completed on the $\mathrm{CaCO}_{3}-\mathrm{MgCO}_{3}, \mathrm{MgCO}_{3}$ $\mathrm{FeCO}_{3}, \mathrm{CaCO}_{3}-\mathrm{FeCO}_{3}, \mathrm{CaCO}_{3}-\mathrm{MnCO}_{3}$, and $\mathrm{CaCO}_{3}-\mathrm{SrCO}_{3}$ systems. Glynn (2000) reviews the thermodynamic theory of aqueous solution-solid solution interactions for systems from $0^{\circ} \mathrm{C}$ to $100^{\circ} \mathrm{C}$, and summarizes the available data on the effects of ionic substitution on the thermodynamic properties of selected rhombohedral (calcite) and orthorhombic (aragonite) carbonate structure solid solutions.

Examples of important carbonate solid solutions include $\mathrm{Mg}$ (or magnesian) calcites that involve the random substitution of a range of $\mathrm{Mg}$ for $\mathrm{Ca}$ in the calcite rhombohedral structure. Magnesian calcites have been the subject of extensive study and research interest. Biogenic calcites may contain up to $30 \mathrm{~mol} \% \mathrm{Mg}$ in solid solution. Magnesian calcites are usually described as either low Mg-calcites with less than $5 \mathrm{~mol} \% \mathrm{Mg}$, or high Mg-calcites with 5-30 mol\% Mg (Appelo and Postma 2005). Mackenzie et al. (1983) and Morse and Mackenzie (1990) review the occurrence of low-temperature magnesian calcites and their chemistry, mineralogy, and composition controls in natural systems.

A complete solid solution also exists in the substitution of $\mathrm{Fe}^{2+}$ for $\mathrm{Mg}^{2+}$ in the siderite $\left(\mathrm{FeCO}_{3}\right)$ magnesite $\left(\mathrm{MgCO}_{3}\right)$ system. Fe-Mg carbonates are common in sedimentary systems. Chai and Navrotsky (1996b) have studied the synthesis, characterization, and mixing properties of the $(\mathrm{Fe}, \mathrm{Mg}) \mathrm{CO}_{3}$ solid solution.

Dolomite also exhibits solid solutions. The Ca-to-Mg ratio may vary and substitution of $\mathrm{Fe}$ and $\mathrm{Mn}$ is common in dolomites. Unlike the siderite-magnesite solid solution series, $\mathrm{Fe}^{2+}$ only partially substitutes for $\mathrm{Mg}^{2+}$ in dolomite to form ankerite $\left[\mathrm{Ca}\left(\mathrm{Fe}_{\mathrm{x}} \mathrm{Mg}_{1-\mathrm{x}}\right)\left(\mathrm{CO}_{3}\right)_{2}\right.$, where $\mathrm{x}$ varies from 0 to 0.7 (Reeder 1983a). The end-member compound $\mathrm{CaFe}\left(\mathrm{CO}_{3}\right)_{2}$ does not occur naturally nor has been synthesized in the laboratory (Reeder 1983a). Carbonates within this solid solution range have been called ankerites and ferroan dolomites. These two terms are often used, respectively, to the more and less Fe rich solidsolution phases. The boundary between dolomite and ankerite is arbitrarily set at $20 \mathrm{~mol} \% \mathrm{CaFe}\left(\mathrm{CO}_{3}\right)_{2}$. Chai and Navrotsky (1996a) have studied the synthesis, characterization, and energetics along the dolomite-ankerite join.

It is not possible from the literature reviewed in this report to assess the importance of solid solutions relative to $\mathrm{CO}_{2}$ sequestration processes and mineral trapping reactions. Solid-solution carbonates were reported in some of the natural analogue and laboratory fluid-rock studies reviewed in our assessment (Table 7.1). These include the identification of ferroan calcite (Worden 2006), magnesian calcite (Park and Fan 2004), ferroan and magnesian carbonates (Watson et al. 2004), and ferroan dolomite (Pauwels et al. 2007; Pearce et al. 1996; Worden 2006). Xu et al. (2004, 2005, 2007) (see Table 5.1) assumed an ideal solution for the dolomite-ankerite $\left[\mathrm{Ca}\left(\mathrm{Fe}_{07} \mathrm{Mg} 0.3\right)\left(\mathrm{CO}_{3}\right)_{2}\right]$ solid solution in their modeling of $\mathrm{CO}_{2}$ sequestration processes using the reactive geochemical transport code TOUGHREACT. Zwingmann et al. (2005) used the geochemical model EQ3/6 to model $\mathrm{CO}_{2}$ sequestration in a sandstone formation in 
Japan. As part of the initial mineralogical composition they used for the injection formation, Zwingmann et al. (2005) included a calcite solid solution with the composition ( $\mathrm{Ca}, \mathrm{Mg}, \mathrm{Fe}, \mathrm{Zn}, \mathrm{Sr}, \mathrm{Mn}) \mathrm{CO}_{3}$, with the six end members being calcite, magnesite, siderite, smithsonite, strontianite, and rhodochrosite. It is not clear from the results of these modeling studies as to the relative importance the carbonate solid solutions had to final modeling results. Given how common such solid solutions are in nature, it is assumed inclusion of carbonate solid solutions would be paramount to accurately modeling $\mathrm{CO}_{2}$ sequestration systems. 


\subsection{Importance of Pitzer Approach to Modeling $\mathrm{CO}_{2}$ Sequestration Applications}

Geochemical modeling of sequestration of $\mathrm{CO}_{2}$ in deep saline formations has received particular attention because of their widespread extent, large storage capacity, and typically good permeability. Unfortunately, methods such as the Debye-Hückel and Davies equations, commonly used to calculate activity coefficients for aqueous species, are not typically sufficient and will lead to inaccurate modeling results in these high-ionic strength systems. Chemical reactions are written in terms of effective concentrations or activities and not the concentrations of dissolved species. The difference between the activity and dissolved concentration of a dissolved species is by definition expressed as the activity coefficient $\gamma_{\mathrm{i}}$. Two general approaches are used in geochemical reaction models to calculate activity coefficients of aqueous species. These methods, their equation forms, the history of their derivation, and their use in calculating activity coefficients are discussed in detail in sources such as Bethke (2008), Langmuir (1997), Marini (2007), and Nordstrom and Munoz (1985), and will not be repeated here. Bethke (2008) and Marini (2007) also provide example calculations to demonstrate the issues related to using these different approaches to calculate geochemical reactions in high-ionic strength solutions.

The first type consists of the Debye-Hückel equation, its variant the Davies equation, and its extended B-dot equation form. This approach treats the activity coefficient of a species as function of the ion size and ionic strength of the solution. These equations are easy to implement and are available in almost all geochemical models. Although this approach takes into account the distribution of species in solution, these particular approaches are, however, typically limited in accuracy to modeling relative dilutions solutions with ionic strengths less than that of seawater. The Debye-Hückel and Davies equation are typically accurate to concentrations of approximately 0.1 and $0.3-0.5$ molal, respectively (Bethke 2008), and the references therein. Bethke (2008) lists the accuracy of the B-dot model to ionic strengths of several molal for predicting the activities of $\mathrm{Na}^{+}$and $\mathrm{Cl}^{-}$ions and to approximately 0.3 to 1 molal for the activities of other species.

The second approach involves the use of virial equations. These semi-empirical equations are sometimes called specific interaction equations or Pitzer equations after Kenneth Pitzer (Pitzer 1973) who was primarily responsible for their development. The Pitzer equations are linear algebraic functions of $\ln$ $\gamma_{\mathrm{i}}$. They differ conceptually from other approaches in that coefficients are used to account for interactions between the individual dissolved components rather than species. This approach is more complicated to apply, requires considerable amounts of data, involves numerous individual parameters, and provides little insight into the distribution of aqueous species (Bethke 2008). The virial method is reviewed in detail in Pitzer (1987) and Weare (1987). A particularly noteworthy implementation of this approach was its use by Harvie et al. (1984) for geochemical modeling mineral solubilities in the Na-K-Mg-Ca-H-Cl$\mathrm{SO}_{4}-\mathrm{OH}-\mathrm{HCO}_{3}-\mathrm{CO}_{3}-\mathrm{CO}_{2}-\mathrm{H}_{2} \mathrm{O}$ system to high-ionic strengths. Their implementation of this method is commonly known as the Harvie-Møller-Weare model. The parameters for the Harvie-Møller-Weare model are listed in Weare (1987). Plummer et al. (1988) also includes a large, comprehensive list of virial coefficients. Examples of recent applications of the Pitzer equations to calculations of carbonate mineral solubilities include De Visscher and Vanderdeelen (2003), Gledhill and Morse (2006), and Marion (2001).

Because virial equations can be applied accurately to systems to high-ionic strengths such as brines and highly saline groundwaters, Pitzer equations have been incorporated into several geochemical 
reactions models. These include the models EQ3/EQ6 (Wolery 1992), Geochemist's Workbench ${ }^{\circledR}$ (Bethke and Yeakel 2007), PHREEQC (Parkhurst 1995), PHRQPITZ (Plummer et al. 1988), and SOLMINEQ.88 (Kharaka et al. 1988).

Given the limitations of the Debye-Hückel and Davies equations for calculating geochemical reactions at high-ionic strengths, application of geochemical models that include the Pitzer equations is important to accurately model $\mathrm{CO}_{2}$ sequestration in saline formations. However, because redox reactions, especially for the $\mathrm{Fe}(\mathrm{II}) / \mathrm{Fe}$ (III) system, and Al system have not been parameterized for the Pitzer model, use of the Pitzer method currently will not adequately address Fe- or Al-containing geochemical reactions associated with modeling $\mathrm{CO}_{2}$ field sites and results from fluid-rock experiments. The importance of using Pitzer equations for modeling reactions in saline waters, such as those targeted as possible host formations for $\mathrm{CO}_{2}$ injection and storage, and the need for parameterization of redox, $\mathrm{Fe}$, and $\mathrm{Al}$ species are recognized by most geochemical modelers, but is nonetheless a potential limitation to accurately model such systems. 


\subsection{Conclusions}

Geochemical reaction models require thermodynamic data to calculate the geochemical reactions associated with $\mathrm{CO}_{2}$ sequestration. Availability of accurate and complete thermodynamic data is certainly a critical data requirement for the successful use of geochemical reaction modeling to $\mathrm{CO}_{2}$ sequestration field site and laboratory applications (Gaus et al. 2008; Marini 2007).

The results of the literature review indicated that an extensive thermodynamic database exists for $\mathrm{CO}_{2}$ and $\mathrm{CH}_{4}$ gases, carbonate aqueous species, and carbonate minerals. Specifically, thermodynamic data exist in databases used for several commonly used geochemical reaction models and/or in published compilations for the following:

\begin{tabular}{|c|c|c|}
\hline Aqueous Species & & Gases \\
\hline $\mathrm{CO}_{2}^{\circ}(\mathrm{aq})$ & $\mathrm{Fe}^{\mathrm{III}} \mathrm{CO}_{3}^{+}$ & $\mathrm{CO}_{2}(\mathrm{~g})$ \\
\hline $\mathrm{CH}_{4}^{\circ}(\mathrm{aq})$ & $\mathrm{Fe}^{\mathrm{II}} \mathrm{HCO}_{3}^{+}$ & $\mathrm{CH}_{4}(\mathrm{~g})$ \\
\hline $\mathrm{CO}_{3}{ }^{2-}$ & $\mathrm{MgCO}_{3}{ }^{\circ}(\mathrm{aq})$ & \\
\hline $\mathrm{HCO}_{3}^{-}$ & $\mathrm{MgHCO}_{3}^{+}$ & \\
\hline $\mathrm{H}_{2} \mathrm{CO}_{3}{ }^{\circ}(\mathrm{aq})$ & $\mathrm{MnCO}_{3}{ }^{\circ}(\mathrm{aq})$ & \\
\hline $\mathrm{CaCO}_{3}{ }^{\circ}(\mathrm{aq})$ & $\mathrm{MnHCO}_{3}^{+}$ & \\
\hline $\mathrm{CaHCO}_{3}^{+}$ & $\mathrm{NaCO}_{3}^{-}$ & \\
\hline $\mathrm{Fe}^{\mathrm{II}} \mathrm{CO}_{3}{ }^{\circ}(\mathrm{aq})$ & $\mathrm{NaHCO}_{3}^{\circ}(\mathrm{aq})$ & \\
\hline
\end{tabular}

\section{Minerals and Related Solids}

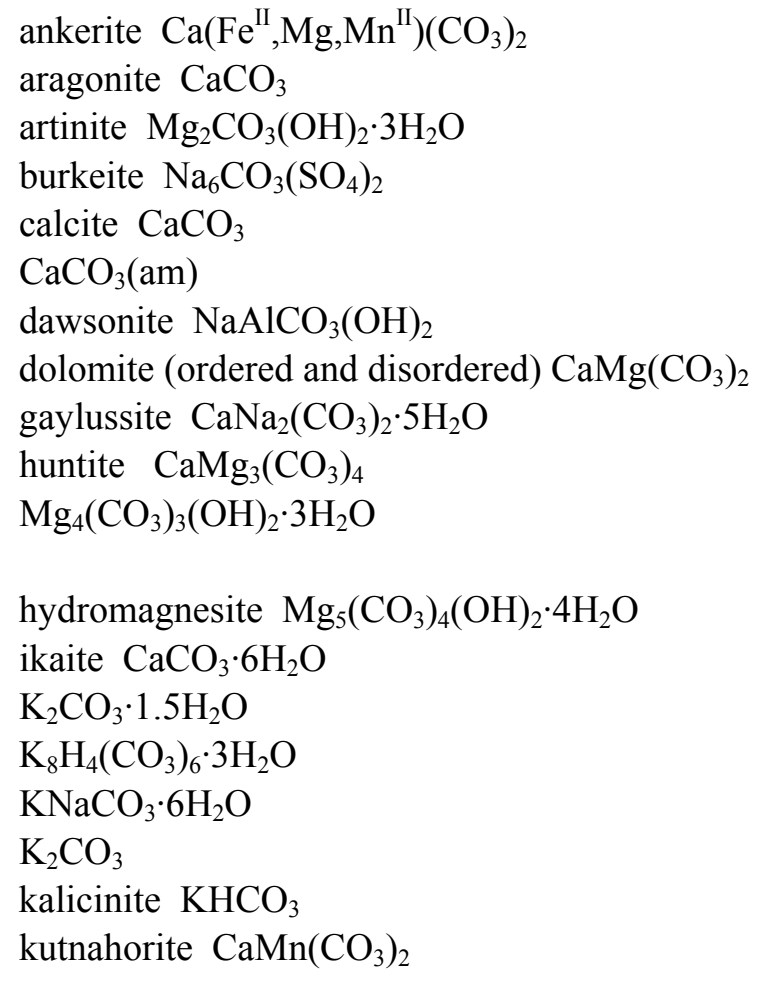

lansfordite $\mathrm{MgCO}_{3} \cdot 5 \mathrm{H}_{2} \mathrm{O}$

magnesite $\mathrm{MgCO}_{3}$

meionite (Al/Si ordered) $\mathrm{Ca}_{4} \mathrm{Al}_{6} \mathrm{Si}_{6} \mathrm{O}_{24} \mathrm{CO}_{3}$

monohydrocalcite $\mathrm{CaCO}_{3} \cdot \mathrm{H}_{2} \mathrm{O}$

$\mathrm{Na}_{2} \mathrm{CO}_{3}$ (likely natrite)

$\mathrm{Na}_{2} \mathrm{CO}_{3} \cdot 7 \mathrm{H}_{2} \mathrm{O}$

nahcolite $\mathrm{NaHCO}_{3}$

natron $\mathrm{Na}_{2} \mathrm{CO}_{3} \cdot 10 \mathrm{H}_{2} \mathrm{O}$

nesquehonite $\mathrm{MgCO}_{3} \cdot 3 \mathrm{H}_{2} \mathrm{O}$

pirssonite $\mathrm{Na}_{2} \mathrm{Ca}\left(\mathrm{CO}_{3}\right)_{2} \cdot 2 \mathrm{H}_{2} \mathrm{O}$

rhodochrosite $\mathrm{MnCO}_{3}$ (natural and synthetic

forms)

siderite $\mathrm{FeCO}_{3}$ (natural and precipitated forms)

spurrite $\mathrm{Ca}_{5} \mathrm{Si}_{2} \mathrm{O}_{8} \mathrm{CO}_{3}$

thermonatrite $\mathrm{Na}_{2} \mathrm{CO}_{3} \cdot \mathrm{H}_{2} \mathrm{O}$

tilleyite $\mathrm{Ca}_{5} \mathrm{Si}_{2} \mathrm{O}_{7}\left(\mathrm{CO}_{3}\right)_{2}$

trona $\mathrm{Na}_{3} \mathrm{H}\left(\mathrm{CO}_{3}\right)_{2} \cdot 2 \mathrm{H}_{2} \mathrm{O}$

trona- $\mathrm{K} \mathrm{K}_{2} \mathrm{NaH}\left(\mathrm{CO}_{3}\right)_{2} \cdot 2 \mathrm{H}_{2} \mathrm{O}$

vaterite $\mathrm{CaCO}_{3}$

wegscheiderite $\mathrm{Na}_{5}\left(\mathrm{HCO}_{3}\right)_{3} \mathrm{CO}_{3}$ 
Values of $\Delta_{\mathrm{f}} \mathrm{G}_{298}{ }^{\circ}$ and $/$ or $\log \mathrm{K}_{\mathrm{r}, 298}{ }^{\circ}$ are available for essentially all of these compounds. $\log \mathrm{K}_{\mathrm{r}, \mathrm{T}}{ }^{\circ}$ or heat capacity values at temperatures above $298 \mathrm{~K}$ exist for less than approximately one-third of these compounds. For those compounds that do not have high-temperature heat capacity data, the tabulated high-temperature $\log \mathrm{K}_{\mathrm{r}, \mathrm{T}}{ }^{\circ}$ are likely based on extrapolation methods that relied on thermodynamic properties reported at $298 \mathrm{~K}\left(25^{\circ} \mathrm{C}\right)$. Because the temperatures of host formations that will be used for $\mathrm{CO}_{2}$ injection and sequestration and of the supporting geochemical reaction modeling studies will be at temperatures in the range of $50^{\circ} \mathrm{C}$ to $100^{\circ} \mathrm{C}$ or greater, the lack of high-temperature thermodynamic values for key carbonate compounds-especially minerals-will likely impact the accuracy of some modeling calculations.

In comparison to the available thermodynamic data for carbonate minerals, the list is short for the carbonate minerals that were described in published studies as being actually present in the host rock formations at $\mathrm{CO}_{2}$ injection test sites or natural analogue sites, reaction products of $\mathrm{CO}_{2}$ injection/ intrusion into a host formation, or as mineral reactants or products of laboratory $\mathrm{CO}_{2}$ fluid-rock/-mineral experiments. Most of the carbonate mineralogical data reported in the literature were from natural analogue site studies and fluid-rock/mineral laboratory experiments.

The list of identified carbonate minerals contains typically common carbonate minerals, including ankerite, aragonite, calcite, ferroan and magnesian calcites, dawsonite, dolomite, ferroan dolomite, magnesite, and siderite. Except for ferroan and magnesian calcites and ferroan dolomite, values are available for the key thermodynamic parameters of the remaining of these carbonate minerals. This suggests that the thermodynamic data for the pure common carbonate minerals is at least adequate for geochemical reaction modeling of $\mathrm{CO}_{2}$ applications. However, Railsback (1999) noted that more thorough mineralogical characterization in the future might show greater diversity of carbonate mineralogy in geological systems affected by anthropogenic activities.

Carbonate mineral solid solutions are common and have been studied extensively. Examples of important carbonate solid solutions include magnesian calcites that involve substitution of $\mathrm{Mg}$ for $\mathrm{Ca}$ in the calcite rhombohedral structure. Complete solid solution also exists in the substitution of $\mathrm{Fe}^{2+} \mathrm{for}_{\mathrm{Mg}^{2+}}$ in the siderite $\left(\mathrm{FeCO}_{3}\right)$-magnesite $\left(\mathrm{MgCO}_{3}\right)$ system. Dolomite also exhibits solid solutions. The Ca-to$\mathrm{Mg}$ ratio may vary and substitution of $\mathrm{Fe}$ and $\mathrm{Mn}$ is common in dolomites. Unlike the siderite-magnesite solid solution series, $\mathrm{Fe}^{2+}$ only partially substitutes for $\mathrm{Mg}^{2+}$ in dolomite to form ankerite $\left[\mathrm{Ca}\left(\mathrm{Fe}_{\mathrm{x}} \mathrm{Mg}_{1-\mathrm{x}}\right)\right.$ $\left(\mathrm{CO}_{3}\right)_{2}$ ], where $\mathrm{x}$ varies from 0 to 0.7 (Reeder 1983a).

It is not possible from the literature captured by this review to assess the importance of solid solutions to adequately model $\mathrm{CO}_{2}$ sequestration processes and mineral trapping reactions. Solid-solution carbonates, such as ferroan and magnesian carbonates and ferroan dolomite, were reported in some of the natural analogue and laboratory fluid-rock studies reviewed in our assessment. Solid-solution carbonate models were also used in a few of the geochemical reaction modeling studies identified from our literature review, but it is not clear if their inclusion had an impact on the final modeling results. Given how common carbonate solid solutions are in nature, one would have to assume inclusion of carbonate solid solutions would be paramount to accurately modeling $\mathrm{CO}_{2}$ sequestration systems.

Two general approaches are used in geochemical reaction models to calculate activity coefficients of aqueous species. The first type consists of the Debye-Hückel equation, its variant the Davies equation, and its extended B-dot equation form. These equations are easy to implement and are available in almost all geochemical models. These equations are, however, typically limited in accuracy to modeling 
relatively dilute solutions with ionic strengths less than that of seawater. Therefore, their use in modeling sequestration of $\mathrm{CO}_{2}$ in deep saline formations would likely lead to inaccurate results for these high-ionic strength waters. The second approach involves the use of virial equations, which are sometimes called specific interaction equations or Pitzer equations. This approach is more complicated to apply, requires considerable amounts of data, and involves numerous individual parameters. Pitzer equations can be applied accurately to systems to high-ionic strengths such as brines and highly saline groundwaters, and have been incorporated into several geochemical reactions models, such as EQ3/EQ6 (Wolery 1992), Geochemist's Workbench ${ }^{\circledR}$, PHREEQC, PHRQPITZ, and SOLMINEQ.88. Given the limitations of the Debye-Hückel and Davies equations for calculating geochemical reactions at high-ionic strengths, application of geochemical models that include the Pitzer equations is important to accurate geochemical reaction modeling in some $\mathrm{CO}_{2}$ sequestration systems. However, because redox reactions, especially for the $\mathrm{Fe}(\mathrm{II}) / \mathrm{Fe}$ (III) system, and $\mathrm{Al}$ system have not been fully parameterized for the Pitzer model, use of the Pitzer method currently will not adequately address Fe- or Al-containing geochemical reactions

associated with modeling certain $\mathrm{CO}_{2}$ sequestration geochemical systems. The importance of using Pitzer equations for modeling reactions in saline waters and the need for parameterization of redox, $\mathrm{Fe}$, and $\mathrm{Al}$ species are recognized by modelers, but is nonetheless a potential limitation to accurately model such systems. 


\subsection{References}

Alemu BL, P Aaagard, E Skurtveit, and IA Munz. 2009. "Effect of $\mathrm{CO}_{2}$ Interaction with Svalbard Shale: Implications to Caprock Integrity for Subsurface Sequestration of $\mathrm{CO}_{2}$." Geochimica et Cosmochimica Acta 73(13 Supplement 1):A23.

Alfredsson HA and SR Gislason. 2009. "CarbFix - $\mathrm{CO}_{2}$ Sequestration in Basaltic Rock: Chemistry of the Rocks and Waters at the Injection Site, Hellisheidi, SW-Iceland." Geochimica et Cosmochimica Acta 73(13 Supplement 1):A26.

Allen DE, BR Strazisar, Y Soong, and SW Hedges. 2005. "Modeling Carbon Dioxide Sequestration in Saline Aquifers: Significance of Elevated Pressures and Salinities." Fuel Processing Technology 86(14-15):1569-1580.

André L, P Audigane, M Azaroual, and A Menjoz. 2007. "Numerical Modeling of Fluid-Rock Chemical Interactions at the Supercritical $\mathrm{CO}_{2}$-Liquid Interface during $\mathrm{CO}_{2}$ Injection into a Carbonate Reservoir, the Dogger Aquifer (Paris Basin, France)." Energy Conversion and Management 48(6):1782-1797.

Andreani M, P Gouze, L Luquot, and P Jouanna. 2008. "Changes in Seal Capacity of Fractured Claystone Caprocks Induced by Dissolved and Gaseous $\mathrm{CO}_{2}$ Seepage." Geophysical Research Letters 35:L14404 (6 pp.).

Andreani M, L Luquot, P Gouze, M Godard, E Hoisé, and B Gibert. 2009. "Experimental Study of Carbon Sequestration Reactions Controlled by the Percolation of $\mathrm{CO}_{2}$-Rich Brine through Peridotites." Environmental Science and Technology 43(4):1226-1231.

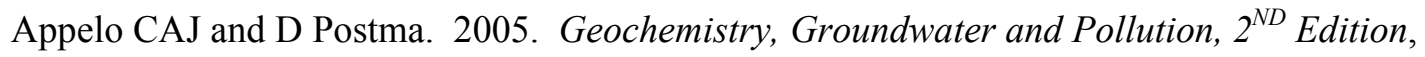
A.A. Balkema Publishers, New York, New York.

Assayag N, J Matter, M Ader, D Goldberg, and P Agrinier. 2008. " $\mathrm{CO}_{2}$ Ionic Trapping by Water-Rock Interactions during a Push-Pull Test in a Basaltic-Metasedimentary Aquifer." Geochimica et Cosmochimica Acta 72(12 Supplement 1):A36.

Assayaga N, J Matter, M Ader, D Goldberg, and P Agriniera. 2009. "Water-Rock Interactions during a $\mathrm{CO}_{2}$ Injection Field-Test: Implications on Host Rock Dissolution and Alteration Effects." Chemical Geology 262(3-4):406-414.

Audigane P, I Gaus, I Czernichowski-Lauriol, K Pruess, and T Xu. 2007. “Two-Dimensional Reactive Transport Modeling of $\mathrm{CO}_{2}$ Injection in a Saline Aquifer at the Sleipner Site, North Sea." American Journal of Science 307(7):974-1008.

Azaroual M, C Kerveévan, M-V Durance, and P Durst. 2004. "SCALE2000: Reaction-Transport Software Dedicated to Thermo-Kinetic Prediction and Quantification of Scales - Applicability to Desalination Problems." Desalination 165(1-3):409-419. 
Bachu S and JJ Adams. 2003. "Sequestration of $\mathrm{CO}_{2}$ in Geological Media in Response to Climate Change: Capacity of Deep Saline Aquifers to Sequester $\mathrm{CO}_{2}$ in Solution." Energy Conversion and Management 44(20):3151-3175.

Bachu S and DB Bennion. 2009. "Experimental Assessment of Brine and/or $\mathrm{CO}_{2}$ Leakage through Well Cements at Reservoir Conditions." International Journal of Greenhouse Gas Control 3(4):494-501.

Bachu S, WD Gunter, and EH Perkins. 1994. "Aquifer Disposal of $\mathrm{CO}_{2}-$ Hydrodynamic and Mineral Trapping." Energy Conversion and Management 35(4):269-279.

Baines SJ and RH Worden. 2004. "The Long-Term Fate of $\mathrm{CO}_{2}$ in the Subsurface: Natural Analogues for $\mathrm{CO}_{2}$ Storage." In Geological Storage of Carbon Dioxide, SJ Baines and RH Worden (eds.), p. 59-85, Geological Society Special Publication No. 233, Geological Society of London, Long, England.

Banfield JF and KH Nealson (eds.). 1997. Reviews in Mineralogy, Volume 35. Geomicrobiology: Interactions between Microbes and Minerals, Mineralogical Society of America, Washington, D.C.

Barlet-Gouédard V, G Rimmelé, B Goffé, and O Porcherie. 2007. "Well Technologies for $\mathrm{CO}_{2}$ Geological Storage: $\mathrm{CO}_{2}$-Resistant Cement." Oil and Gas Science and Technology - Rev. IFP 62(3):325-334.

Barlet-Gouédard V, G Rimmelé, O Porcherie, N Quisel, and J Desroches. 2009. “A Solution against Well Cement Degradation under $\mathrm{CO}_{2}$ Geological Storage Environment." International Journal of Greenhouse Gas Control 3(2):206-216.

Bateman K, G Turner, JM Pearce, DJ Noy, D Birchall, and CA Rochelle. 2005. "Large-Scale Column Experiment: Study of $\mathrm{CO}_{2}$, Porewater, Rock Reactions and Model Test Case." Oil and Gas Science and Technology - Rev. IFP 60(1):161-175.

Béarat H, MJ McKelvy, AVG Chizmeshya, D Gormley, R Nunez, RW Carpenter, K Squires, and GH Wolf. 2006. "Carbon Sequestration via Aqueous Olivine Mineral Carbonation: Role of Passivating Layer Formation.” Environmental Science and Technology 40(15):4802-4808.

Beinlich A, A Austrheim, J Glodny, M Erambert, and TB Andersen. 2009. " $\mathrm{CO}_{2}$ Sequestration and Extreme Mg Leaching in Serpentinized Peridotite Clasts of the Solund Devonian Basin, SW-Norway." Geochimica et Cosmochimica Acta 73(13 Supplement 1):A105.

Bénézeth P, JL Dandurand, and JC Harrichoury. 2009a. "Solubility Product of Siderite $\left(\mathrm{FeCO}_{3}\right)$ as a Function of Temperature (25-250 $\left.{ }^{\circ} \mathrm{C}\right)$." Chemical Geology 265(1-2):3-12.

Bénézeth P, GD Saldi, J Schott, and JL Dandurand. 2009b. "Siderite, Magnesite and Dolomite Solubility Products as a Function of Temperature." Geochimica et Cosmochimica Acta 73(13 Supplement 1):A108.

Benson SM and DR Cole. 2008. " $\mathrm{CO}_{2}$ Sequestration in Deep Sedimentary Formations." Elements 4(5):325-331.

Bentham M and M Kirby. 2005. " $\mathrm{CO}_{2}$ Storage in Saline Aquifers." Oil and Gas Science and Technology - Rev. IFP 60(3):559-567. 
Bertier P, R Swennen, and B Laenen. 2007. "Evaluation of the $\mathrm{CO}_{2}$-Sequestration Capacity of Sandstone Aquifers in the Campine Basin (NE-Belgium) Based on Autoclave Experiments and Numerical Modelling." Geochimica et Cosmochimica Acta 71(15 Supplement S):A87.

Bertier P, R Swennen, B Laenen, D Lagrou, and R Dreesen. 2006. "Experimental Identification of $\mathrm{CO}_{2}$ Water-Rock Interactions Caused by Sequestration of $\mathrm{CO}_{2}$ in Westphalian and Buntsandstein Sandstones of the Campine Basin (NE-Belgium)." Journal of Geochemical Exploration 89(1-3):10-14.

Bethke CM . 2008. Geochemical and Biochemical Reaction Modeling, $2^{\text {nd }}$ Edition, Oxford University Press, New York, New York.

Bethke CM and S Yeakel. 2007. The Geochemist's Workbench ${ }^{\circledR}$, Version 7.0, GWB Essentials Guide, Hydrogeology Program, University of Illinois, Urbana, Illinois.

Boschi C, A Dini, L Dallai, G Ruggieri, and G Gianelli. 2009. "Enhanced $\mathrm{CO}_{2}$-Mineral Sequestration by Cyclic Hydraulic Fracturing and Si-Rich Fluid Infiltration into Serpentinites at Malentrata (Tuscany, Italy)." Chemical Geology 265(1-2):209-226.

Brosse É, C Magnier, and B Vincent. 2005. "Modelling Fluid-Rock Interaction Induced by the Percolation of $\mathrm{CO}_{2}$-Enriched Solutions in Core Samples: The Role of Reactive Surface Area." Oil and Gas Science and Technology - Rev. IFP 60(2):287-305.

Brunet F, J Corvisier, A Fabbri, S Bernard, N Findling, G Rimmele, V Barlet-Gouedard, O Beyssac, and B Goffe. 2009. "Calcium Carbonates Distribution in Experimentally Carbonated Portland Cement Cores." Geochimica et Cosmochimica Acta 73(13 Supplement 1):A166.

Bruno J, D Bosbach, D Kulik, and A Navrotsky. 2007. Chemical Thermodynamics Series Volume 10. Chemical Thermodynamics of Solid Solutions of Interest in Nuclear Waste Management, OECD Publishing, Paris, France.

Bruno J, F Grandia, and E Vilanova. 2009. "Trace Element Behaviour in Connection to the Geological Storage of $\mathrm{CO}_{2}$ : Lessons from Natural Analogues." Geochimica et Cosmochimica Acta 73(13 Supplement 1):A167.

Busch A, S Alles, Y Gensterblum, D Prinz, DN Dewhurst, MD Raven, H Stanjek, and BM Krooss. 2008. "Carbon Dioxide Storage Potential of Shales." International Journal of Greenhouse Gas Control 2(3):297-308.

Buschkuehle BE and EH Perkins. 2004. "Mineralogy and Geochemical Trapping of Acid Gas in the Edmonton Area of Central Alberta, Canada." 7th International Conference on Greenhouse Gas Control Technologies, Elsevier Limited, United Kingdom.

Cantucci B, G Montegrossi, M Buttinelli, F Lucci, O Vaselli, and F Quattrocchi. 2009a. "Overview of the Geochemical Modeling on $\mathrm{CO}_{2}$ Capture and Storage in Italian Feasibility Studies." Geochimica et Cosmochimica Acta 73(13 Supplement 1):A190. 
Cantucci B, G Montegrossi, O Vaselli, F Tassi, F Quattrocchi, and EH Perkins. 2009b. "Geochemical Modeling of $\mathrm{CO}_{2}$ Storage in Deep Reservoirs: The Weyburn Project (Canada) Case Study." Chemical Geology 265(1-2):181-197.

Carey JW and PC Lichtner. 2007. "Calcium Silicate Hydrate (C-S-H) Solid Solution Model Applied to Cement Degradation Using the Continuum Reactive Transport Model FLOTRAN.” In Transport Properties and Concrete Quality: Materials Science of Concrete, B Mobasher and J Skalny (eds.), p. 73-106, Special Volume, American Ceramic Society, John Wiley and Sons, New York, New York.

Carey JW, M Wigand, SJ Chipera, G WoldeGabriel, R Pawar, PC Lichtner, SC Wehner, MA Raines, and GDJ Guthrie. 2007. "Analysis and Performance of Oil Well Cement with 30 Years of $\mathrm{CO}_{2}$ Exposure from the SACROC Unit, West Texas, USA.” International Journal of Greenhouse Gas Control $1(1): 75-85$.

Carlson WD. 1983. "The Polymorphs of $\mathrm{CaCO}_{3}$ and the Aragonite-Calcite Transformation." In Reviews in Mineralogy. Volume 11. Carbonates: Mineralogy and Chemistry, RJ Reeder (ed.), p. 191-225, Mineralogical Society of America, Washington, D.C.

Carmichael ISE and HP Eugster (eds.). 1987. Reviews in Mineralogy, Volume 17. Thermodynamic Modeling of Geological Materials: Minerals, Fluids and Melts, Mineralogical Society of America, Washington, D.C.

Celia MA and S Bachu. 2003. "Geological Sequestration of CO2: Is Leakage Unavoidable and Acceptable?" In Proceedings of the Sixth International Conference on Greenhouse Gas Control Technologies, J Gale and Y Kaya (eds.), p. 477-482, 1, Elsevier Science Limited, Kidlington, Oxford, United Kingdom.

Centeno J, A Ramírez, and A Blanco. 2009. "SEM-EDS Applications in Mineralogical Phases Study of Oil Well Cements Attacked with $\mathrm{H}_{2} \mathrm{~S} / \mathrm{CO}_{2}$ Mixtures." Geochimica et Cosmochimica Acta 73(13 Supplement 1):A203.

Centeno J, A Ramirez, A Blanco, and A Balza. 2007. "Durability and Degradation of Oil Well Cement Exposed to a Source of $\mathrm{H}_{2} \mathrm{~S}$ and $\mathrm{CO}_{2}$ Gases." Geochimica et Cosmochimica Acta 71(15 Supplement S):A154.

Centeno J, A Ramirez, A Colina, and A Blanco. 2005. "Dissolution of Oil Well Cement in Presence of $\mathrm{CO}_{2} / \mathrm{H}_{2} \mathrm{~S}$ under HTHP.” Geochimica et Cosmochimica Acta 69(10 Supplement S):A355.

Chai L and A Navrotsky. 1996a. "Synthesis, Characterization, and Energetics of Solid Solution along the Dolomite-Ankerite Join, and Implications for the Stability of Ordered $\mathrm{CaFe}\left(\mathrm{CO}_{3}\right)_{2}$." American Mineralogist 81(9-10):1141-1147.

Chai L and A Navrotsky. 1996b. "Synthesis, Characterization, and Enthalpy of Mixing of the (Fe, $\mathrm{Mg}) \mathrm{CO}_{3}$ Solid Solution.” Geochimica et Cosmochimica Acta 60(22):4377-4383.

Charan SN and Z Begum. 2008. "Geological Sequestration of Carbon-Dioxide in Deccan Continental Flood Basalt Province, India.” Geochimica et Cosmochimica Acta 72(12 Supplement 1):A150. 
Charan SN, PSR Prasad, and DS Sarma. 2009. "Mineral Trapping in Deccan Basalt Province India: Implications for Geological Sequestration of $\mathrm{CO}_{2}$." Geochimica et Cosmochimica Acta 73(13 Supplement 1):A209.

Choi W, H Kang, J Lee, and M Lee. 2009. "Dissolution of Anorthite under Supercritical $\mathrm{CO}_{2}$ - Water Mineral Reaction System at a $\mathrm{CO}_{2}$ Sequestration Site." Geochimica et Cosmochimica Acta 73(13 Supplement 1):A222.

Cipolli F, B Gambardella, L Marini, G Ottonello, and M Vetuschi Zuccolini. 2004. "Geochemistry of High-pH Waters from Serpentinites of the Gruppo di Voltri (Genova, Italy) and Reaction Path Modeling of $\mathrm{CO}_{2}$ Sequestration in Serpentinite Aquifers." Applied Geochemistry 19(5):787-802.

Cölfen H. 2003. "Precipitation of Carbonates: Recent Progress in Controlled Production of Complex Shapes." Current Opinion in Colloid and Interface Science 8(1):23-31.

Corvisier J, F Brunet, A Fabbri, S Bernard, N Findling, G Rimmelé, V Barlet-Gouédard, O Beyssac, and B Goffé. 2010. "Raman Mapping and Numerical Simulation of Calcium Carbonates Distribution in Experimentally Carbonated Portland-Cement Cores." European Journal of Minteralogy 22(1):63-74.

Corvisier J, F Brunet, V Morales-Florez, and B Goffe. 2009. "A New Thermodynamic Model for C-S-H Solubility Valid in the Range of P-T Conditions Encountered around $\mathrm{CO}_{2}$-Injection Wells." Geochimica et Cosmochimica Acta 73(13 Supplement 1):A244.

Daval D, I Martinez, J Corvisier, N Findling, B Goffé, and F Guyot. 2009. "Carbonation of Ca-Bearing Silicates, the Case of Wollastonite: Experimental Investigations and Kinetic Modeling." Chemical Geology 265(1-2):63-78.

De Visscher A and J Vanderdeelen. 2003. "Estimation of the Solubility Constant of Calcite, Aragonite, and Vaterite at $25^{\circ} \mathrm{C}$ Based on Primary Data Using the Pitzer Ion Interaction Approach." Monatshefte für Chemie 134(5):769-775.

Dilmore RM, DE Allen, JR McCarthy Jones, SW Hedges, and Y Soong. 2008a. "Sequestration of Dissolved $\mathrm{CO}_{2}$ in the Oriskany Formation." Environmental Science and Technology 42(8):2760-2766.

Dilmore RM, P Lu, D Allen, Y Soong, S Hedges, JK Fu, CL Dobbs, A Degalbo, and C Zhu. 2008b. "Sequestration of $\mathrm{CO}_{2}$ in Mixtures of Bauxite Residue and Saline Wastewater." Energy and Fuels 22(1):343-353.

Duan $\mathrm{ZH}$ and R Sun. 2003. "An Improved Model Calculating $\mathrm{CO}_{2}$ Solubility in Pure Water and Aqueous $\mathrm{NaCl}$ Solutions from 273 to $533 \mathrm{~K}$ and from 0 to 2000 Bar." Chemical Geology 193(3-4):257-271.

Dufaud F, I Martinez, and S Shilobreeva. 2009. "Experimental Study of Mg-Rich Silicates Carbonation at 400 and $500{ }^{\circ} \mathrm{C}$ and 1 kbar." Chemical Geology 265(1-2):79-87.

Dupraz S, B Ménez, P Gouze, R Leprovost, P Bénézeth, OS Pokrovsky, and F Guyot. 2009a. "Experimental Approach of $\mathrm{CO}_{2}$ Biomineralization in Deep Saline Aquifers." Chemical Geology 265(1-2):54-62. 
Dupraz S, M Parmentier, B Ménez, and F Guyot. 2009b. "Experimental and Numerical Modeling of Bacterially Induced pH Increase and Calcite Precipitation in Saline Aquifers." Chemical Geology 265(1-2):44-53.

Dupraz S, M Papmentier, B Menez, P Gouze, R Leprovost, and F Guyot. 2007. "A Bacterial Model for Studying Interactions between Microorganisms and $\mathrm{CO}_{2}$ Injected in the Subsurface." Geochimica et Cosmochimica Acta 71(15 Supplement S):A244.

Dupraz S, M Parmentier, B Menez, and F Guyot. 2008. "Experimental Study of Biomineralization Processes Relevant to $\mathrm{CO}_{2}$ Geological Sequestration." Geochimica et Cosmochimica Acta 72(12 Supplement 1):A233.

Emberley S, I Hutcheon, M Shevalier, K Durocher, WD Gunter, and EH Perkins. 2004. “Geochemical Monitoring of Fluid-Rock Interaction and $\mathrm{CO}_{2}$ Storage at the Weyburn $\mathrm{CO}_{2}$-Injection Enhanced Oil Recovery Site, Saskatchewan, Canada.” Energy 29(9-10):1393-1401.

Emberley S, I Hutcheon, M Shevalier, K Durocher, B Mayer, WD Gunter, and EH Perkins. 2005. "Monitoring of Fluid-Rock Interaction and $\mathrm{CO}_{2}$ Storage Through Produced Fluid Sampling at the Weyburn $\mathrm{CO}_{2}$-Injection Enhanced Oil Recovery Site, Saskatchewan, Canada." Applied Geochemistry 20(6):1131-1157.

Enick RM and SM Klara. 1990. " $\mathrm{CO}_{2}$ Solubility in Water and Brine under Reservoir Conditions." Chemical Engineering Communications 90:23-33.

Ennis-King J and L Paterson. 2007. "Coupling of Geochemical Reactions and Convective Mixing in the Long-Term Geological Storage of Carbon Dioxide." International Journal of Greenhouse Gas Control $1(1): 86-93$.

Fernandez-Bastero S, C Gil-Lozano, MJI Briones, and L Gago-Duport. 2008. "Kinetic and Structural Constraints during Glauconite Dissolution: Implications for Mineral Disposal of $\mathrm{CO}_{2}$." Mineralogical Magazine 72(1):27-31.

Flaathen TK and SR Gislason. 2007. "The Groundwater Beneath Hekla Volcano, Iceland; A Natural Analogue for $\mathrm{CO}_{2}$ Sequestration." Geochimica et Cosmochimica Acta 71(15 Supplement S):A283.

Flaathen TK, SR Gislason, EH Oelkers, and AE Sveinbjörnsdóttir. 2009. "Chemical Evolution of the Mt. Hekla, Iceland, Groundwaters: A Natural Analogue for $\mathrm{CO}_{2}$ Sequestration in Basaltic Rocks." Applied Geochemistry 24(3):463-474.

Flukiger F and D Bernard. 2009. "A New Numerical Model for Pore Scale Dissolution of Calcite due to $\mathrm{CO}_{2}$ Saturated Water Flow in 3D Realistic Geometry: Principles and First Results." Chemical Geology 265(1-2):171-180.

Frost RL, S Bahfenne, and J Graham. 2008a. "Infrared and Infrared Emission Spectroscopic Study of Selected Magnesium Carbonate Minerals Containing Ferric Iron - Implications for the Geosequestration of Greenhouse Gases." Spectrochimica Acta Part A - Molecular and Biomolecular Spectroscopy 71(4):1610-1616. 
Frost RL, S Bahfenne, J Graham, and WN Martens. 2008b. "Thermal Stability of Artinite, Dypingite and Brugnatellite - Implications for the Geosequestration of Green House Gases." Thermochimica Acta 475(1-2):39-43.

Garcia B, V Beaumont, D Blanchet, P Oger, G Dromart, A-Y Huc and F Haeseler. 2008. "Basic Minerals for Carbon Dioxide Sequestration: Potential and Quantification." Geochimica et Cosmochimica Acta 72(12 Supplement 1):A296.

Gaus I, P Audigane, L Andre, J Lions, N Jacquemet, P Dutst, I Czernichowski-Lauriol, and M Azaroual. 2008. "Geochemical and Solute Transport Modelling for $\mathrm{CO}_{2}$ Storage, What to Expect from It?" International Journal of Greenhouse Gas Control 2(4-Special Issue SI):605-625.

Gaus I, M Azaroual, and I Czernichowski-Lauriol. 2005. "Reactive Transport Modelling of the Impact of $\mathrm{CO}_{2}$ Injection on the Clayey Cap Rock at Sleipner (North Sea)." Chemical Geology 217(3-4):319-337.

Gherardi F, T Xu, and K Pruess. 2007. "Numerical Modeling of Self-Limiting and Self-Enhancing Caprock Alteration Induced by $\mathrm{CO}_{2}$ Storage in a Depleted Gas Reservoir." Chemical Geology 244(1-2):103-129.

Gislason SR, D Wolff-Boenisch, A Stefansson, EH Oelkers, E Gunnlaugsson, H Sigurdardottir, B Sigfusson, WS Broecker, J Matter, M Stute, G Axelsson, and T Fridriksson. 2010. "Mineral Sequestration of Carbon Dioxide in Basalt: A Pre-Injection Overview of the Carbfix Project." International Journal of Greenhouse Gas Control 4(3):537-545.

Gislason SR, WS Broecker, EH Oelkers, E Gunnlaugsson, H Sigurdardottir, A Stefansson, D WolffBoenisch, J Matter, M Stute, and G Axelsson. 2009. "The Carbfix Project: Mineral $\mathrm{CO}_{2}$ Sequestration into Basalt." Geochimica et Cosmochimica Acta 73(13 Supplement 1):A440.

Gledhill DK and JW Morse. 2006. "Calcite Solubility in Na-Ca-Mg-Cl Brines." Chemical Geology 233(3-4):249-256.

Glynn PD. 1990. "Modeling Solid-Solution Reactions in Low-Temperature Aqueous Systems." In Chemical Modeling in Aqueous Systems II, RL Bassett and D Melchior (eds.), p. 74-86, Symposium Series 416, American Chemical Society, Washington D.C.

Glynn PD. 2000. "Solid-Solution Solubilities and Thermodynamics - Sulfates, Carbonates, and Halides." In Reviews in Mineralogy and Geochemistry. Volume 40. Sulfate Minerals - Crystallography, Geochemistry, and Environmental Significance, CN Alpers, JL Jambor, and DK Nordstrom (eds.), p. 281-511, Mineralogical Society of America, Washington, D.C.

Goldberg DS, T Takahashi, and AL Slagle. 2008. "Carbon Dioxide Sequestration in Deep-Sea Basalt." Proceedings of the National Academy of Sciences of the United States of America 105(29):9920-9925.

Goldsmith JR. 1983. "Phase Relations of Rhombohedral Carbonates." In Reviews in Mineralogy. Volume 11. Carbonates: Mineralogy and Chemistry, RJ Reeder (ed.), p. 49-76, Mineralogical Society of America, Washington, D.C. 
Gudbrandsson S, D Wolff-Boenisch, SR Gislason, and EH Oelkers. 2009. "Dissolution Rates of Crystalline Basalt as a Function of Temperature and Solution Composition." Geochimica et Cosmochimica Acta 73(13 Supplement 1):A474.

Guichet X, J Schott, EH Oelkers, B Vincent, C Magnier, and E Brosse. 2005. "Reactive Transport Experiments and Modelling of $\mathrm{CO}_{2}$ Sequestration in Deep Aquifers." Geochimica et Cosmochimica Acta 69(10 Supplement S):A179.

Gunter WD, EH Perkins, and I Hutcheon. 2000. “Aquifer Disposal of Acid Gases: Modelling of WaterRock Reactions for Trapping of Acid Wastes." Applied Geochemistry 15(8):1085-1095.

Gunter WD, B Wiwchar, and EH Perkins. 1997. "Aquifer Disposal of $\mathrm{CO}_{2}$-Rich Greenhouse Gases: Extension of the Time Scale of Experiment for $\mathrm{CO}_{2}$-Sequestering Reactions by Geochemical Modelling." Mineralogy and Petrology 59:121-140.

Gysi AP and A Stefánsson. 2008. "Numerical Modelling of $\mathrm{CO}_{2}$-Water-Basalt Interaction." Mineralogical Magazine 72(1):55-59.

Gysi AP and A Stefánsson. 2009. " $\mathrm{CO}_{2}$-Water-Basalt Interaction: Geochemical Modelling and Experiments." Geochimica et Cosmochimica Acta 73(13 Supplement 1):A483.

Haas JL and JR Fisher. 1976. "Simultaneous Evaluation and Correlation of Thermodynamic Data." American Journal of Science 276(4):525-545.

Hänchen M, V Prigiobbe, R Baciocchi, and M Mazzotti. 2008. "Precipitation in the Mg-Carbonate System - Effects of Temperature and $\mathrm{CO}_{2}$ Pressure." Chemical Engineering Science 63(4):1012-1028.

Hangx SJT and CJ Spiers. 2009. "Reaction of Plagioclase Feldspars with $\mathrm{CO}_{2}$ under Hydrothermal Conditions." Chemical Geology 265(1-2):88-98.

Hansen LD, GM Dipple, TM Gordon, and DA Kellett. 2005. "Carbonated Serpentinite (Listwanite) at Atlin, British Columbia: A Geological Analogue to Carbon Dioxide Sequestration.” Canadian Mineralogist 43(Part 1):225-239.

Harvie CE, N Møller, and JH Weare. 1984. "The Prediction of Mineral Solubilities in Natural Waters: The Na-K-Mg-Ca-H-Cl-SO $\mathrm{S}_{4}-\mathrm{OH}-\mathrm{HCO}_{3}-\mathrm{CO}_{3}-\mathrm{CO}_{2}-\mathrm{H}_{2} \mathrm{O}$ System to High Ionic Strengths at $25^{\circ} \mathrm{C}$." Geochimica et Cosmochimica Acta 48(4):723-751.

Haszeldine ES, O Quinn, G England, M Wilkinson, ZK Shipton, JP Evans, J Heath, L Crossey, CJ Ballentine, and CM Graham. 2005. "Natural Geochemical Analogues for Carbon Dioxide Storage in Deep Geological Porous Reservoirs, a United Kingdom Perspective.” Oil and Gas Science and Technology - Rev. IFP 60(1):33-49.

Holland TJB and R Powell. 1998. "An Internally Consistent Thermodynamic Data Set for Phases of Petrological Interest.” Journal of Metamorphic Geology 16(3):309-343.

Hövelmann J and H Austrheim. 2009. "Guidelines for Experiments on $\mathrm{CO}_{2}$ Sequestration in Peridotites based on a Natural Example.” Geochimica et Cosmochimica Acta 73(13 Supplement 1):A539. 
Huet B, R Fuller, J Prevost, and G Scherer. 2006. "Development of a Geochemical Code to Assess Cement Reactivity in $\mathrm{CO}_{2}$ /Brine Mixtures." In CO2SC 2006 - International Symposium on Site Characterization for $\mathrm{CO}_{2}$ Geological Storage, Lawrence Berkeley National Laboratory, Berkeley, California, March 20-22, 2006. Proceedings of Extended Abstracts, p. 164-168, Lawrence Berkeley National Laboratory, Berkeley, California (http://esd.lbl.gov/CO2SC/).

Hummel W, U Berner, E Curti, FJ Pearson, and T Thoenen. 2002. Nagra/PSI Chemical Thermodynamic Data Base 01/01, Universal Publishers, Parkland, Florida.

Hurter S, D Labregere, and J Berge. 2007. "Simulations of Dry-Out and Halite Precipitation due to $\mathrm{CO}_{2}$ Injection." Geochimica et Cosmochimica Acta 71(15 Supplement S):A426.

Iglesias RS, JM Ketzer, S Einloft, J Dullius, R Ligabue, V Lima, and DC Silva. 2008. "Experimental and Modeling Studies of $\mathrm{CO}_{2}$-Water-Rock Interactions in the Rio Bonito Saline Aquifer, Paraná Basin, Brazil." Geochimica et Cosmochimica Acta 72(12 Supplement 1):A406.

Izgec O, B Demiral, $\mathrm{H}$ Bertin, and S Akin. 2008a. " $\mathrm{CO}_{2}$ Injection into Saline Carbonate Aquifer Formations I: Laboratory Investigation.” Transport in Porous Media 72(1):1-24.

Izgec O, B Demiral, H Bertin, and S Akin. 2008b. " $\mathrm{CO}_{2}$ Injection into Saline Carbonate Aquifer Formations II: Comparison of Numerical Simulations to Experiments." Transport in Porous Media 73(1):57-74.

Jacquemet N, J Pironon, and E Caroli. 2005. "A New Experimental Procedure for Simulation of $\mathrm{H}_{2} \mathrm{~S}+\mathrm{CO}_{2}$ Geological Storage. Application to Well Cement Aging." Oil and Gas Science and Technology - Rev. IFP 60(1):193-206.

Jacquemet N, J Pironon, and J Saint-Marc. 2008. "Mineralogical Changes of a Well Cement in Various $\mathrm{H}_{2} \mathrm{~S}-\mathrm{CO}_{2}$ (-Brine) Fluids at High Pressure and Temperature." Environmental Science and Technology 42(1):282-288.

Jarvis K, RW Carpenter, T Windman, Y Kim, R Nunez, and F Alawneh. 2009. "Reaction Mechanisms for Enhancing Mineral Sequestration of $\mathrm{CO}_{2}$." Environmental Science and Technology 43(16):6314-6319.

Jiménez-López C, E Caballero, FJ Huertas, and CS Romanek. 2001. "Chemical, Mineralogical and Isotope Behavior, and Phase Transformation during the Precipitation of Calcium Carbonate Minerals from Intermediate Ionic Solution at 25 ${ }^{\circ}$ C." Geochimica et Cosmochimica Acta 65(19):3219-3231.

Johnson JW, JJ Nitao, and KG Knauss. 2004. "Reactive Transport Modelling of $\mathrm{CO}_{2}$ Storage in Saline Aquifers to Elucidate Fundamental Processes, Trapping Mechanisms, and Sequestration Partitioning." In Geological Storage of Carbon Dioxide, SJ Baines and RH Worden (eds.), p. 10-128, Geological Society Special Publication No. 233, Geological Society of London, London, England.

Johnson JW, JJ Nitao, CI Steelfel, and KG Knauss. 2001. Reactive Transport Modeling of Geologic $\mathrm{CO}_{2}$ Sequestration in Saline Aquifers: The Influence of Intra-Aquifer Shales and the Relative Effectiveness of Structural, Solubility, and Mineral Trapping During Prograde and Retrograde Sequestration.

UCRL-JC-146932, Lawrence Livermore National Laboratory, Livermore, California. 
Johnson JW, EH Oelkers, and HC Helgeson. 1992. “SUPCRT92: A Software Package for Calculating the Standard Molal Thermodynamic Properties of Minerals, Gases, Aqueous Species, and Reactions from 1 to 5000 bar and 0 to $1000^{\circ}$ C." Computers and Geosciences 18(7):899-947.

Kaszuba JP, DR Janecky, and MG Snow. 2003. "Carbon Dioxide Reaction Processes in a Model Brine Aquifer at $200^{\circ} \mathrm{C}$ and 200 Bars: Implications for Geologic Sequestration of Carbon." Applied Geochemistry 18(7):1065-1080.

Kaszuba JP, DR Janecky, and MG Snow. 2005. "Experimental Evaluation of Mixed Fluid Reactions between Supercritical Carbon Dioxide and $\mathrm{NaCl}$ Brine: Relevance to the Integrity of a Geologic Carbon Repository." Chemical Geology 217(3-4):277-293.

Kervévan C, M Azaroual, and P Durst. 2005. "Improvement of the Calculation Accuracy of Acid Gas Solubility in Deep Reservoir Brines: Application to the Geological Storage of $\mathrm{CO}_{2}$." Oil and Gas Science and Technology - Rev. IFP 60(2):357-379.

Ketzer JM, R Iglesias, S Einloft, J Dullius, R Ligabue, and V de Lima. 2009. "Water-Rock-CO $\mathrm{CO}_{2}$ Interactions in Saline Aquifers Aimed for Carbon Dioxide Storage: Experimental and Numerical Modeling Studies of the Rio Bonito Formation (Permian), Southern Brazil." Applied Geochemistry 24(5):760-767.

Kharaka YK, DR Cole, SD Hovorka, WD Gunter, KG Knauss, and BM Freifeld. 2006a. "Gas-WaterRock Interactions in Frio Formation Following $\mathrm{CO}_{2}$ Injection: Implications for the Storage of Greenhouse Gases in Sedimentary Basins.” Geology 34(7):577-580.

Kharaka YK, DR Cole, JJ Thordsen, E Kakouros, and HS Nance. 2006b. "Gas-Water-Rock Interactions in Sedimentary Basins: $\mathrm{CO}_{2}$ Sequestration in the Frio Formation, Texas, USA." Journal of Geochemical Exploration 89(1-3):183-186.

Kharaka YK, WD Gunter, PK Aggarwal, EH Perkins, and JD DeBraal. 1988. SOLMINEQ.88, A Computer Program for Geochemical Modeling of Water-Rock Interactions. USGS Water Resources Investigations Report 88-4227, U.S. Geological Survey, Menlo Park, California.

Kharaka YK, JJ Thordsen, SD Hovorka, DR Cole, TJ Phelps, and TD Bullen. 2007. "Potential Environmental Issues of $\mathrm{CO}_{2}$ Storage in Saline Aquifers: Geochemical Results from the Frio Brine Pilot Tests, Texas, USA.” Geochimica et Cosmochimica Acta 71(15 Supplement S):A481.

Kitamura M. 2001. "Crystallization and Transformation Mechanism of Calcium Carbonate Polymorphs and the Effect of Magnesium Ion.” Journal of Colloid and Interface Science 236(2):318-327.

Knauss KG, JW Johnson, and CI Steefel. 2005. "Evaluation of the Impact of $\mathrm{CO}_{2}$, Co-contaminant Gas, Aqueous Fluid and Reservoir Rock Interactions on the Geologic Sequestration of $\mathrm{CO}_{2}$." Chemical Geology 217(3-4):339-350.

Krupka KM, BS Hemingway, RA Robie, and DM Kerrick. 1985. "High-Temperature Heat-Capacities and Derived Thermodynamic Properties of Anthophyllite, Diopside, Dolomite, Enstatite, Bronzite, Talc, Tremolite, and Wollastonite." American Mineralogist 70(3-4):261-271. 
Kühn M and C Clauser. 2007. "Reactive Transport Simulation of Mineral Trapping of $\mathrm{CO}_{2}$ in Operated Geothermal Aquifers." Geochimica et Cosmochimica Acta 71 (15 Supplement S):A529.

Kunieda M, Y Yamada, A Ueda, and T Matsuoka. 2008. "Experimental Research of Casing Cement Alteration by Interaction with Supercritical $\mathrm{CO}_{2}$ for Geological Sequestration." Geochimica et Cosmochimica Acta 72(12 Supplement 1):A503.

Kuroda Y, Y Yamada, A Ueda, and T Matsuoka. 2008. "Experimental Research of Plagioclase-GasWater Interaction at Hydrothermal Conditions Caused by $\mathrm{CO}_{2}$ Sequestration." Geochimica et Cosmochimica Acta 72(12 Supplement 1):A506.

Kutchko BG, BR Strazisar, DA Dzombak, GV Lowry, and N Thaulow. 2007. "Degradation of Well Cement by $\mathrm{CO}_{2}$ under Geologic Sequestration Conditions." Environmental Science and Technology 41(13):4787- 4792.

Kutchko BG, BR Strazisar, N Huerta, GV Lowry, DA Dzombak, and N Thaulow. 2009. " $\mathrm{CO}_{2}$ Reaction with Hydrated Class H Well Cement under Geologic Sequestration Conditions: Effects of Flyash Admixtures." Environmental Science and Technology 43(10):3947-3952.

Kutchko BG, BR Strazisar, GV Lowry, DA Dzombak, and N Thaulow. 2008. "Rate of $\mathrm{CO}_{2} \mathrm{Attack}$ on Hydrated Class H Well Cement under Geologic Sequestration Conditions." Environmental Science and Technology 42(16):6237-6242.

Lagneau V, A Pipart, and H Catalette. 2005. "Reactive Transport Modelling of $\mathrm{CO}_{2}$ Sequestration in Saline Aquifers." Oil and Gas Science and Technology - Rev. IFP 60(2):231-247.

Langmuir D. 1997. Aqueous Environmental Geochemistry, Prentice Hall, Upper Saddle River, New Jersey.

Le Gallo Y, O Bildstein, and Y Brosse. 1998. “Coupled Reaction-Flow Modeling of Diagenetic Changes in Reservoir Permeability, Porosity and Mineral Compositions." Journal of Hydrology 209:366-388.

Lin H, T Fujii, R Takisawa, T Takahashi, and T Hashida. 2008. "Experimental Evaluation of Interactions in Supercritical $\mathrm{CO}_{2} /$ Water/Rock Minerals System under Geologic $\mathrm{CO}_{2}$ Sequestration Conditions." Journal of Materials Science 43:2307-2315.

Liu L, Y Suto, G Bignall, N Yamasaki, and T Hashida. 2003. " $\mathrm{CO}_{2}$ Injection to Granite and Sandstone in Experimental Rock/Hot Water Systems." Energy Conversion and Management 44:399-1410.

Loeppert RH, AP Schwab, and S Goldberg (eds.). 1995. Chemical Equilibrium and Reaction Models, Soil Science Society of America, Inc., Madison, Wisconsin.

Luquot L and P Gouze. 2009. "Experimental Determination of Porosity and Permeability Changes Induced by Injection of $\mathrm{CO}_{2}$ into Carbonate Rocks.” Chemical Geology 265(1-2):148-159.

Machel HG. 2005. "Geological and Hydrogeological Evaluation of the Nisku Q-Pool in Alberta, Canada, for $\mathrm{H}_{2} \mathrm{~S}$ and/or $\mathrm{CO}_{2}$ Storage." Oil and Gas Science and Technology - Rev. IFP 60(1):51-65. 
Mackenzie FT, WD Bischoff, FC Bishop, M Loijens, J Schoonmaker, and R Wollast. 1983. "Magnesian Calcites: Low-Temperature Occurrence, Solubility and Solid Solution Behavior." In Reviews in Mineralogy. Volume 11. Carbonates: Mineralogy and Chemistry, RJ Reeder (ed.), p. 97-144, Mineralogical Society of America, Washington, D.C.

Madé B, A Clément, and B Fritz. 1994. "Modeling Mineral Solution Interactions - The Thermodynamic and Kinetic Code KINDISP.”Computers and Geosciences 20(9):1347-1363.

Maier CG and KK Kelly. 1932. "An Equation for the Representation of High Temperature Heat Content Data." Journal of the American Chemical Society 54:3243-3246.

Mandarino JA and ME Back. 2004. Fleischer's Glossary of Mineral Species 2004, The Mineralogical Record Inc., Tucson, Arizona.

Mani D, SN Charan, and B Kumar. 2008. "Assessment of Carbon Dioxide Sequestration Potential of Ultramafic Rocks in the Greenstone Belts of Southern India.” Current Science 94(1):53-60.

Marini L. 2007. Geological Sequestration of Carbon Dioxide: Thermodynamics, Kinetics, and Reaction Path Modeling (Developments in Geochemistry 11), Elsevier, New York, New York.

Marion GM. 2001. "Carbonate Mineral Solubility at Low Temperatures in the Na-K-Mg-Ca-H-Cl-SO $\mathrm{OH}-\mathrm{HCO}_{3}-\mathrm{CO}_{3}-\mathrm{CO}_{2}-\mathrm{H}_{2} \mathrm{O}$ System.” Geochimica et Cosmochimica Acta 65(12):1883-1896.

Martinez RE, OS Pokrovsky, J Schott, and EH Oelkers. 2007. "Characterization of Carbonate Mineral Formation by Cyanobacteria and the Implications in $\mathrm{CO}_{2}$ Sequestration." Geochimica et Cosmochimica Acta 71(15 Supplement S):A628.

Matter JM and PB Kelemen. 2009. "Enhanced In Situ Carbonation of Peridotite for Permanent $\mathrm{CO}_{2}$ Storage." Geochimica et Cosmochimica Acta 73(13 Supplement 1):A848.

May F. 2005. "Alteration of Wall Rocks by $\mathrm{CO}_{2}$-Rich Water Ascending in Fault Zones: Natural Analogues for Reactions Induced by $\mathrm{CO}_{2}$ Migrating along Faults in Siliciclastic Reservoir and Cap Rocks." Oil and Gas Science and Technology - Rev. IFP 60(1):19-32.

McGrail BP, HT Schaef, AM Ho, Y-J Chien, JJ Dooley, and CL Davidson. 2006. "Potential for Carbon Dioxide Sequestration in Flood Basalts." Journal of Geophysical Research B. Solid Earth, Vol. 111:B12201 (13 pp.).

McKelvy MJ, AVG Chizmeshya, J Diefenbacher, H Béarat, and G Wolf. 2004. "Exploration of the Role of Heat Activation in Enhancing Serpentine Carbon Sequestration Reactions." Environmental Science and Technology 38(24):6897-6903.

Mitchell AC, AJ Phillips, R Hiebert, R Gerlach, LH Spangler, and AB Cunningham. 2009. "Biofilm Enhanced Geologic Sequestration of Supercritical $\mathrm{CO}_{2}$." International Journal of Greenhouse Gas Control 3(1):90-99. 
Mitchell AC, AJ Phillips, JP Kaszuba, WK Hollis, ALB Cunningham, and R Gerlach. 2008. "Microbially Enhanced Carbonate Mineralization and the Geologic Containment of $\mathrm{CO}_{2}$." Geochimica et Cosmochimica Acta 72(12 Supplement 1):A636.

Michael K and BEM Buschkuehle. 2006. "Acid-Gas Injection at West Stoddart, British Columbia: An Analogue for the Detailed Hydrogeological Characterization of a $\mathrm{CO}_{2}$ Sequestration Site." Journal of Geochemical Exploration 89(1-3):280-283.

Mito S and K Nakagawa. 2009. "Influence of Formation Water Composition on Mineral Trapping of $\mathrm{CO}_{2}$." Geochimica et Cosmochimica Acta 73(13 Supplement 1):A887.

Mito S, Z Xue, and T Ohsumi. 2008. "Case Study of Geochemical Reactions at the Nagaoka $\mathrm{CO}_{2}$ Injection Site, Japan.” International Journal of Greenhouse Gas Control 2(3):309-318.

Moore J, M Adams, R Allis, S Lutz, and S Rauzi. 2005. "Mineralogical and Geochemical Consequences of the Long-Term Presence of $\mathrm{CO}_{2}$ in Natural Reservoirs: An Example from the Springerville-St. Johns Field, Arizona, and New Mexico, USA.” Chemical Geology 217(3-4):365-385.

Morse JW and FT Mackenzie. 1990. Geochemistry of Sedimentary Carbonates, Developments in Sedimentology 48, Elsevier, New York, New York.

Morse JW, DW Finneran, K Walton, and RS Arvidson. 2008. "Calcite Nucleation and Epitaxial Growth from High Ionic Strength $\mathrm{NaCl}$ Solutions." Geochimica et Cosmochimica Acta 72(12 Supplement 1):A653.

Munemoto T and K Fukushi. 2009. "Solubility of Monohydrocalcite between 5 to $40^{\circ} \mathrm{C}$." Geochimica et Cosmochimica Acta 73(13 Supplement 1):A916.

Navrotsky A. 1987. "Models of Crystalline Solutions." In Reviews in Mineralogy. Volume 17. Thermodynamic Modeling of geological Materials: Minerals, Fluids and Melts, ISE Carmichael and HP Eugster (eds.), p. 35-69, Mineralogical Society of America, Washington, D.C.

Nissenbaum J, SLS Stipp, and A Johnnson. 2008. "Transformation of Calcium Carbonate Polymorphs: Preliminary Results.” Mineralogical Magazine 72(1):473-476.

Noiriel C, L Luquot, B Madé, L Raimbault, P Gouze, and J van der Lee. 2009. "Changes in Reactive Surface Area during Limestone Dissolution: An Experimental and Modelling Study." Chemical Geology 265(1-2):160-170.

Nordbotten JM, MA Celia, S Bachu, and HK Dahle. 2005. "Semianalytical Solution for $\mathrm{CO}_{2}$ Leakage through an Abandoned Well.” Environmental Science and Technology 39(2):602-611.

Nordstrom DK and JL Munoz. 1985. Geochemical Thermodynamics, The Benjamin/Cummings Publishing Co., Inc., Menlo Park, California. 
Nordstrom DK, LN Plummer, D Langmuir, E Busenberg, HM May, BF Jones, and DL Parkhurst. 1990. "Revised Chemical Equilibrium Data for Major Water-Mineral Reactions and Their Limitations." In Chemical Modeling in Aqueous Systems II, RL Bassett and D Melchior (eds.), p. 398-413, Symposium Series 416, American Chemical Society, Washington, D.C.

Oelkers EH, SR Gislason, and J Matter. 2008. "Mineral Carbonation of $\mathrm{CO}_{2}$." Elements 4(5):333-337.

Ogino T, T Suzuki, and K Sawada. 1987. "The Formation and Transformation Mechanism of CalciumCarbonate in Water." Geochimica et Cosmochimica Acta 51(10):2757-2767.

Ogino T, T Suzuki, and K Sawada. 1990. "The Rate and Mechanism of Polymorphic Transformation of Calcium-Carbonate in Water." Journal of Crystal Growth 100(1-2):159-167.

Ozah RC, S Lakshminarasimhan, GA Pope, K Sepehrnoori, and SL Bryant. 2005. "Numerical Simulation of the Storage of Pure $\mathrm{CO}_{2}$ and $\mathrm{CO}_{2}-\mathrm{H}_{2} \mathrm{~S}$ Gas Mixtures in Deep Saline Aquifers." 2005 SPE Annual Technical Conference and Exhibition, Dallas, TX, October 9-12, 2005, Paper SPE 97255, Society of Petroleum Engineers (SPE), Richardson, Texas.

Palandri JL and YK Kharaka. 2005. "Ferric Iron-Bearing Sediments as a Mineral Trap for $\mathrm{CO}_{2}$ Sequestration: Iron Reduction Using Sulfur-Bearing Waste Gas.” Chemical Geology 217(3-4):351-364.

Palandri JL, RJ Rosenbauer, and YK Kharaka. 2005. "Ferric Iron in Sediments as a Novel $\mathrm{CO}_{2} \mathrm{Mineral}$ Trap: $\mathrm{CO}_{2}-\mathrm{SO}_{2}$ Reaction with Hematite." Applied Geochemistry 20(11):2038-2048.

Park A-HA and L-S Fan. 2004. " $\mathrm{CO}_{2}$ Mineral Sequestration: Physically Activated Dissolution of Serpentine and pH Swing Process.” Chemical Engineering Science 59(22-23):5241-5247.

Parkhurst DL. 1995. User's Guide to PHREEQC, A Computer Program for Speciation, Reaction-Path, Advective-Transport, and Inverse Geochemical Calculations. USGS Water Resources Investigations Report 95-4227, U.S. Geological Survey, Denver, Colorado.

Pauwels H, I Gaus, Y Michel le Nindrea, J Pearceb, and I Czernichowski-Lauriola. 2007. "Chemistry of Fluids from a Natural Analogue for a Geological $\mathrm{CO}_{2}$ Storage Site (Montmiral, France): Lessons for $\mathrm{CO}_{2}$-Water-Rock Interaction Assessment and Monitoring." Applied Geochemistry 22(12):2817-2833.

Pearce JM, S Holloway, H Wacker, MK Nelis, C Rochelle, and K Bateman. 1996. "Natural Occurrences as Analogues for the Geological Disposal of Carbon Dioxide." Energy Conversion and Management 37(6-8):1123-1128.

Perdikouri C, A Kasioptas, CV Putnis, and A Putnis. 2008. "The Effect of Fluid Composition on the Mechanism of the Aragonite to Calcite Transition." Mineralogical Magazine 72(1):111-114.

Pitzer KS. 1973. "Thermodynamics of Electrolytes - I. Theoretical Basis and General Equations." Journal of Physical Chemistry 77:268- 277. 
Pitzer KS. 1987. "Thermodynamic Model for Aqueous Solutions of Liquid-Like Density." In Reviews in Mineralogy. Volume 17. Thermodynamic Modeling of Geological Materials: Minerals, Fluids and Melts, ISE Carmichael and HP Eugster (eds.), p. 97-142, Mineralogical Society of America, Washington, D.C.

Plummer LN, DL Parkhurst, GW Fleming, and SA Dunkle. 1988. PHRQPITZ - A Computer Program Incorporating Pitzer's Equations for Calculation of Geochemical Reactions in Brines. USGS Water Resources Investigations Report 88-4153, U.S. Geological Survey, Reston, Virginia.

Power IM, SA Wilson, JM Thom, GM Dipple, JE Gabites, and G Southam. 2009. "The Hydromagnesite Playas of Atlin, British Columbia, Canada: A Biogeochemical Model for $\mathrm{CO}_{2}$ Sequestration." Chemical Geology 260(3-4):302-316.

Power IM, SA Wilson, JM Thom, GM Dipple, and G Southam. 2007. "Biologically Induced Mineralization of Dypingite by Cyanobacteria from an Alkaline Wetland near Atlin, British Columbia, Canada." Geochemical Transactions 8:Article No. 13 (16 pp.).

Prasad PSR, DS Sarma, L Sudhakar, U Basavaraju, RS Singh, Z Begum, KB Archana, CD Chavan, and SN Charan. 2009. "Geological Sequestration of Carbon Dioxide in Deccan Basalts: Preliminary Laboratory Study.” Current Science 96(2):288-291.

Pruess K, J García, T Kovscek, C Oldenburg, J Rutqvist, C Steefel, and T Xu. 2002. Intercomparison of Numerical Simulation Codes for Geologic Disposal of $\mathrm{CO}_{2}$. LBNL-51813, Lawrence Berkeley National Laboratory Berkeley, California.

Pruess K, J García, T Kovscek, C Oldenburg, J Rutqvist, C Steefel, and T Xu. 2004. “Code Intercomparison Builds Confidence in Numerical Simulation Models for Geologic Disposal of $\mathrm{CO}_{2}$." Energy 29(9-10):1431-1444.

Railsback LB. 1999. "Patterns in the Compositions, Properties, and Geochemistry of Carbonate Minerals." Carbonates and Evaporites 14(1):1-20.

Raistrick M, B Mayer, M Shevalier, RJ Perez, I Hutcheon, E Perkins, and B Gunter. 2006. "Using Chemical and Isotopic Data to Quantify Ionic Trapping of Injected Carbon Dioxide in Oil Field Brines." Environmental Science and Technology 40(21):6744 -6749.

Reed MH and J Palandri. 2006. "Sulfide Mineral Precipitation from Hydrothermal Fluids." In Reviews in Mineralogy and Geochemistry. Volume 61. Sulfide Mineralogy and Geochemistry, DJ Vaughan (ed.), p. 609-631, The Mineralogical Society of America, Chantilly, Virginia.

Reeder RJ. 1983a. "Crystal Chemistry of the Rhombohedral Carbonates." In Reviews in Mineralogy. Volume 11. Carbonates: Mineralogy and Chemistry, RJ Reeder (ed.), p. 1-47, Mineralogical Society of America, Washington, D.C.

Reeder RJ (ed.). 1983b. Reviews in Mineralogy. Volume 11. Carbonates: Mineralogy and Chemistry, Mineralogical Society of America, Washington, D.C. 
Regnault O, V Lagneau, and H Schneider. 2009. "Experimental Measurement of Portlandite Carbonation Kinetics with Supercritical CO $2 . "$ Chemical Geology 265(1-2):113-121.

Rimmelé G, V Barlet-Gouédard, O Porcherie, B Goffé, and F Brunet. 2008. "Heterogeneous Porosity Distribution in Portland Cement Exposed to $\mathrm{CO}_{2}$-Rich Fluids." Cement and Concrete Research 38(8-9):1038-1048.

Robie RA and BS Hemingway. 1972. "The Heat Capacities at Low Temperatures and Entropies at $298.15 \mathrm{~K}$ of Nesquehonite, $\mathrm{MgCO}_{3} \cdot 3 \mathrm{H}_{2} \mathrm{O}$, and Hydromagnesite." American Mineralogist 57(11-12):1768-1781.

Robie RA and GS Hemingway. 1995. Thermodynamic Properties of Minerals and Related Substances at $298.15 \mathrm{~K}$ and 1 bar (10 $0^{5}$ Pascals) Pressure and at Higher Temperatures. U.S. Geological Survey Bulletin 2131, U.S. Geological Survey, Washington, D.C.

Rochelle C, I Czernichowski-Lauriol, and AE Milodowski. 2004. "The Impact of Chemical Reactions on $\mathrm{CO}_{2}$ Storage in Geological Formations: A Brief Review." In Geological Storage of Carbon Dioxide, SJ Baines and RH Worden (eds.), p. 87-106, Geological Society Special Publication No. 233, Geological Society of London, London, England.

Rosenbauer RJ and JL Bischoff. 2009. " $\mathrm{CO}_{2}$ Sequestration by Basalt: Experimental Studies and Geochemical Modeling.” Geochimica et Cosmochimica Acta 73(13 Supplement 1):A1118.

Rosenbauer RJ, T Koksalan, and JL Palandri. 2005. "Experimental Investigation of $\mathrm{CO}_{2}$-Brine-Rock Interactions at Elevated Temperature and Pressure: Implications for $\mathrm{CO}_{2}$ Sequestration in Deep-Saline Aquifers.” Fuel Processing Technology 86(14-15):1581-1597.

Sass B, N Gupta, J Ickes, and S Chattopadhyay. 2001. Geochemical Evaluation of Carbon Dioxide Sequestration in Saline Formations. Final Technical Report, Prepared for the U.S. Department of Energy, National Energy Technology Laboratory by Battelle, Columbus, Ohio.

Sass BM, N Gupta, JA Ickes, MH Engelhard, DR Baer, P Bergman, and C Byrer. 2002. "Interaction of Rock Minerals with Carbon Dioxide and Brine: A Hydrothermal Investigation." Journal of Energy \& Environmental Research (U.S. Department of Energy) 2(1):23-31.

Saylor BZ and B Zerai. 2004. "Injection and Trapping of Carbon Dioxide in Deep Saline Aquifers." In Energy, Waste and the Environment; A Geochemical Perspective, R Gieré and P Stille (eds.), p. 285-296, Special Publications, Volume 236, Geological Society, London, England.

Schaef HT and BP McGrail. 2009. "Dissolution of Columbia River Basalt Under Mildly Acidic Conditions as a Function of Temperature: Experimental Results Relevant to the Geological Sequestration of Carbon Dioxide." Applied Geochemistry 24(5):980-987.

Schaef HT, BP McGrail, and AT Owen. 2010. "Carbonate Mineralization of Volcanic Province Basalts." International Journal of Greenhouse Gas Control 4:249-261. 
Scherer GW, MA Celia, J-H Prévost, S Bachu, R Bruant, A Duguid, R Fuller, SE Gasda, M Radonjic, and W Vichit-Vadakan. 2005. "Leakage of $\mathrm{CO}_{2}$ through Abandoned Wells: Role of Corrosion of Cement." In Carbon Dioxide Capture for Storage in Deep Geologic Formations - Results from the $\mathrm{CO}_{2}$ Capture Project. Volume II, DC Thomas and SM Benson (eds.), p. 827-848, Elsevier Ltd., New York, New York.

Schnaar G and DC Digiulio. 2009. "Computational Modeling of the Geologic Sequestration of Carbon Dioxide." Vadose Zone Journal 8(2):389-403.

Schulze RK, MA Hill, RD Field, PA Papin, RJ Hanrahan, and DD Byler. 2004. "Characterization of Carbonated Serpentine using XPS and TEM.” Energy Conversion and Management 45:3169-3179.

Shannon RD. 1976. "Revised Effective Ionic Radii and Systematic Studies of Interatomic Distances in Halides and Chaleogenides." Acta Crystallographica A32:751-767.

Shilobreeva SN and I Martinez. 2007. " $\mathrm{CO}_{2}$-Water-Basalt Interactions: Experimental and Mineralogical Study." Geochimica et Cosmochimica Acta 71(15 Supplement S):A929.

Shiraki R and TL Dunn. 2000. "Experimental Study on Water-Rock Interactions during $\mathrm{CO}_{2}$ Flooding in the Tensleep Formation, Wyoming, USA." Applied Geochemistry 15(3):265-279.

Smith RM, AE Martell, and RJ Motekaitis. 2004. NIST Critically Selected Stability Constants of Metal Complexes Database. Version 8 for Windows. Users Guide. NIST Standard Reference Database 46, National Institute of Standards and Technology, Gaithersburg, Maryland.

Soong Y, AL Goodman, JR McCarthy-Jones, and JP Baltrus. 2004. "Experimental and Simulation Studies on Mineral Trapping of $\mathrm{CO}_{2}$ with Brine." Energy Conversion and Management 45(11-12):1845-1859.

Speer JA. 1983. "Crystal Chemistry and Phase Relations of Orthorhombic Carbonates." In Reviews in Mineralogy. Volume 11. Carbonates: Mineralogy and Chemistry, RJ Reeder (ed.), p. 145-190, Mineralogical Society of America, Washington, D.C.

Stefánsson A and AP Gysi. 2008. " $\mathrm{CO}_{2}$-Basalt Interaction - Numerical Simulation and Experimental Study." Geochimica et Cosmochimica Acta 72(12 Supplement 1):A894.

Sterpenich J, J Sausse, J Pironon, A Géhin, G Hubert, E Perfetti, and D Grgic. 2009. "Experimental Ageing of Oolitic Limestones under $\mathrm{CO}_{2}$ Storage Conditions: Petrographical and Chemical Evidence." Chemical Geology 265(1-2):99-112.

Stockmann G, D Wolff-Boenisch, SR Gislason, and EH Oelkers. 2008. "Dissolution of Diopside and Basaltic Glass: The Effect of Carbonate Coating.” Mineralogical Magazine 72(1):135-139.

Stockmann GJ, D Wolff-Boenisch, SR Gíslason, and EH Oelkers. 2009. “Can Carbonate Coatings Inhibit In Situ Mineral Carbonation?” Geochimica et Cosmochimica Acta 73(13 Supplement 1):A1277.

Sun W, S Nešić, and RC Woollam. 2009. "The Effect of Temperature and Ionic Strength on Iron Carbonate $\left(\mathrm{FeCO}_{3}\right)$ Solubility Limit.” Corrosion Science 51(6):1273-1276. 
Suto Y, L Liu, N Yamasaki, and T Hashida. 2007. "Initial Behavior of Granite in Response to Injection of $\mathrm{CO}_{2}$-Saturated Fluid." Applied Geochemistry 22(1):202-218.

Teir S, S Eloneva, C-J Fogelholm, and R Zevenhoven. 2009. "Fixation of Carbon Dioxide by Producing Hydromagnesite from Serpentinite.” Applied Energy 86(2-Special Issue SI):214-218.

Testemale D, F Dufaud, I Martinez, P Bénézeth, J-L Hazemann, J Schott, and F Guyot. 2009. “An X-ray Absorption Study of the Dissolution of Siderite at 300 Bar between $50{ }^{\circ} \mathrm{C}$ and $100{ }^{\circ} \mathrm{C}$." Chemical Geology 259(1-2):8-16.

Ueda A, Y Kyduroda, K Sugiyama, A Ozawa, H Wakahama, S Mito, Y Kaji, and H Kaieda. 2009. "Geochemical Monitoring of Calcite Precipitation during $\mathrm{CO}_{2}$ Injection into the Ogachi Hot Dry Rock Site." Geochimica et Cosmochimica Acta 73(13 Supplement 1):A1357.

Ueda A, Y Nakatsuka, M Kunieda, Y Kuroda, K Kato, T Yajima, H Satoh, Y Odashima, K Sugiyama, A Ozawa, T Ohsumi, H Wakahama, S Mito, Y Kaji, and H Kaieda. 2008. "Geochemical Results on the $\mathrm{CO}_{2}$ Georeactor Sequestration Tests at the Ogachi Hot Dry Rock Site, NE Japan." Geochimica et Cosmochimica Acta 72(12 Supplement 1):A965.

Ueda A, T Yajima, H Sato, A Ozawa, H Kaieda, H Ito, T Ohsumi, and K Kato. 2007. "Preliminary Geochemical Results on the $\mathrm{CO}_{2}$ Georeactor Sequestration Test at the Ogachi HDR Site." Geochimica et Cosmochimica Acta 71(15 Supplement S):A1044.

van der Lee J and C Lomenech. 2004. "Towards a Common Thermodynamic Database for Speciation Models." Radiochimica Acta 92(9):811-818.

Vosteen H-D and F May. 2007. "Identification of Potential Geochemical Reactions in German Subsurface Storage Sites of $\mathrm{CO}_{2}$." Geochimica et Cosmochimica Acta 71(15 Supplement S):A1074.

Wagman DD, WH Evans, VB Parker, RH Schumm, I Halow, SM Bailey, KL Churney, and RL Nuttall. 1982. "The NBS Tables of Chemical Thermodynamic Properties, Selected Values for Inorganic and $\mathrm{C}_{1}$ and $\mathrm{C}_{2}$ Organic Substances in SI Units." Journal of Physical and Chemical Reference Data 11(Supplement 2):1-392.

Watson MN, N Zwingmann, and NM Lemon. 2004. "The Ladbroke Grove-Katnook Carbon Dioxide Natural Laboratory: A Recent $\mathrm{CO}_{2}$ Accumulation in a Lithic Sandstone Reservoir." Energy 29(9-10):1457-1466.

Watson TL and S Bachu. 2007. "Evaluation of the Potential for Gas and $\mathrm{CO}_{2}$ Leakage along Wellbores." Paper SPE 106817, Society of Petroleum Engineers, Richardson, Texas. Paper given at E\&P Environmental and Safety Conference, March 5-7, 2007, Galveston, Texas.

Weare JH. 1987. "Models of Mineral Solubility in Concentrated Brines with Application to Field Observations." In Reviews in Mineralogy. Volume 17. Thermodynamic Modeling of Geological Materials: Minerals, Fluids and Melts, ISE Carmichael and HP Eugster (eds.), p. 143-176, Mineralogical Society of America, Washington, D.C. 
Westrich H, J Lorenz, S Cooper, C Jove Colon, N Warpinski, D Zhang, C Bradley, P Lichtner, R Pawa, B Stubbs, R Grigg, R Svec, and C Byrer. 2002. "Sequestration of $\mathrm{CO}_{2}$ in a Depleted Oil Reservoir: An Overview." Journal of Energy and Environmental Research [U.S. Department of Energy, National Energy Technology Laboratory (NETL)] 2(1):64-74.

White SP, RG Allis, J Moore, T Chidsey, C Morgan, W Gwynn, and M Adams. 2005. "Simulation of Reactive Transport of Injected $\mathrm{CO}_{2}$ on the Colorado Plateau, Utah, USA." Chemical Geology 217(3-4):387-405.

Wigand M, JW Carey, H Schütt, E Spangenberg, and J Erzinger. 2008. "Geochemical Effects of $\mathrm{CO}_{2}$ Sequestration in Sandstones under Simulated In Situ Conditions of Deep Saline Aquifers." Applied Geochemistry 23(9):2735-2745.

Wigand M, JP Kaszuba, JW Carey, and WL Hollis. 2009. "Geochemical Effects of $\mathrm{CO}_{2}$ Sequestration on Fractured Wellbore Cement at the Cement/Caprock Interface.” Chemical Geology 265(1-2):122-133.

Wilson SA, GM Dipple, IA Power, JM Thom, RG Anderson, M Raudsepp, JE Gabites, and G Southam. 2009. "Carbon Dioxide Fixation within Mine Wastes of Ultramafic-Hosted Ore Deposits: Examples from the Clinton Creek and Cassiar Chrysotile Deposits, Canada." Economic Geology 104(1):95-112.

Wilson SA, M Raudsepp, and GM Dipple. 2006. "Verifying and Quantifying Carbon Fixation in Minerals from Serpentine-Rich Mine Tailings Using the Rietveld Method with X-ray Powder Diffraction Data." American Mineralogist 91:1331-1341.

Wolery TJ. 1992. EQ3NR, A Computer program for Geochemical Aqueous Speciation-Solubility Calcualtions: Theoretical Manual, User's Guide, and Related Documentation (Version 7.0). UCRL-MA-1 10662 Pt III, Lawrence Livermore National Laboratory, Livermore, California.

Wolf GH, AVG Chizmeshya, J Diefenbacher, and MJ McKelvy. 2004. "In Situ Observation of $\mathrm{CO}_{2}$ Sequestration Reactions Using a Novel Microreaction System.” Environmental Science and Technology 38(3):932-936.

Woods TL and RM Garrels. 1987. Thermodynamic Values at Low Temperature for Natural Inorganic Materials: An Uncritical Summary, Oxford University Press, New York, New York.

Worden RH. 2006. "Dawsonite Cement in the Triassic Lam Formation, Shabwa Basin, Yemen: A Natural Analogue for a Potential Mineral Product of Subsurface $\mathrm{CO}_{2}$ Storage for Greenhouse Gas Reduction." Marine and Petroleum Geology 23(1):61-77.

Xu T, JA Apps, and K Pruess. 2003. "Reactive Geochemical Transport Simulation to Study Mineral Trapping for $\mathrm{CO}_{2}$ Disposal in Deep Arenaceous Formations." Journal of Geophysical Research-Solid Earth 108(B2):Art. No. 2071.

Xu T, E Sonnenthal, N Spycher, and K Pruess. 2006. “TOUGHREACT - A Simulation Program for Non-Isothermal Multiphase Reactive Geochemical Transport in Variably Saturated Geologic Media: Applications to Geothermal Injectivity and $\mathrm{CO}_{2}$ Geological Sequestration." Computers and Geosciences 32(2):145-165. 
$\mathrm{Xu}$ TF, JA Apps, and K Pruess. 2004. "Numerical Simulation of $\mathrm{CO}_{2}$ Disposal by Mineral Trapping in Deep Aquifers.” Applied Geochemistry 19(6):917-936.

Xu TF, JA Apps, and K Pruess. 2005. "Mineral Sequestration of Carbon Dioxide in a Sandstone-Shale System.” Chemical Geology 217(3-4):295-318.

$\mathrm{Xu}$ TF, JA Apps, K Pruess, and H Yamamoto. 2007. "Numerical Modeling of Injection and Mineral Trapping of $\mathrm{CO}_{2}$ with $\mathrm{H}_{2} \mathrm{~S}$ and $\mathrm{SO}_{2}$ in a Sandstone Formation." Chemical Geology 242(3-4):319-346.

Zerai B, BZ Saylor, and G Matisoff. 2006. "Computer Simulation of $\mathrm{CO}_{2}$ Trapped through Mineral Precipitation in the Rose Run Sandstone, Ohio.” Applied Geochemistry 21(2):223-240.

Zhang W, Y Li, T Xu, H Cheng, Y Zheng, and P Xiong. 2009. "Long-Term Variations of $\mathrm{CO}_{2} \mathrm{Trapped}$ in Different Mechanisms in Deep Saline Formations: A Case Study of the Songliao Basin, China." International Journal of Greenhouse Gas Control 3(2):161-180.

Zwingmann N, S Mito, M Sorai, and T Ohsumi. 2005. "Preinjection Characterisation and Evaluation of $\mathrm{CO}_{2}$ Sequestration Potential in the Haizume Formation, Niigata Basin, Japan. Geochemical Modelling of Water-Minerals- $\mathrm{CO}_{2}$ Interaction." Oil and Gas Science and Technology - Rev. IFP 60(2):249-258. 


\section{Appendix}

\section{Carbonate Minerals}




\section{Appendix}

\section{Carbonate Minerals}

The following tables were prepared from the extensive listing of carbonate-containing minerals compiled by L.B. Railsback (Department of Geology, University of Georgia, Athens, Georgia) with help of J. A. Mandarino (Royal Ontario Museum, Toronto, Ontario, Canada). Railsback's tables are provided online at http://www.gly.uga.edu/railsback/CO3mins_intro.html, as an appendix to the paper authored by Railsback (1999). Railsback notes that many of the carbonate-containing minerals listed in his tables would not be classified as carbonate minerals but derive at least part of their negative charge from $\mathrm{CO}_{3}{ }^{2-}$ or $\mathrm{HCO}_{3}^{-}$.

To limit the number of minerals listed in the following tables, we did not include any carbonatecontaining minerals tabulated by Railsback (1999), which include As, B, Ba, Bi, Cd, Ce, Co, Cr, Cu, F, $\mathrm{Ge}, \mathrm{Hg}, \mathrm{La}, \mathrm{Li}, \mathrm{NH}_{4}, \mathrm{Nb}, \mathrm{Nd}, \mathrm{Ni}, \mathrm{Pb}, \mathrm{REE}, \mathrm{Sr}, \mathrm{Ta} \mathrm{U}, \mathrm{Y} \mathrm{Zn}$, and/or Zr. This was done for the sake of convenience and an assumed lack of importance of such carbonate-species to $\mathrm{CO}_{2}$ sequestration. However, it should be noted that carbonate-containing minerals containing transition metals and noncarbonate anions may exert important geochemical controls on the solution concentrations and environmental mobility of these metals and non-carbonate anions even though the mineral makes up a small weight percentage of the total mineralogy of the aquifer system.

$\mathrm{X}$-ray diffraction (XRD) is commonly used to identify crystalline compounds, such as minerals which are by definition crystalline, that are present in mineral, sediment, soil, and rock samples from field site and laboratory $\mathrm{CO}_{2}$ sequestration studies. To determine what minerals are present in a sample, the diffraction peaks in an XRD pattern measured for a sample are compared to the diffraction peaks listed in database patterns for specific minerals of interest. If a sample contains a crystalline compound that does not have an XRD database pattern, it is unlikely the unknown compound will be identified by standard mineral characterization methods. Therefore, to adequately characterize carbonate minerals that might be in samples from field site and laboratory $\mathrm{CO}_{2}$ sequestration studies, it is essential to know to what extent XRD database patterns may be missing for the carbonate minerals in Tables A.1 through A.8. ${ }^{1}$ The carbonate minerals in Tables A.1 through A.8 were therefore checked against the carbonate-containing compounds contained in the mineral powder diffraction files (PDFTM) published by the Joint Committee on Powder Diffraction Standards (JCPDS) International Center for Diffraction Data (ICDD). This evaluation revealed that the ICDD database did not have XRD patterns for only 14 minerals in Tables A.1 through A.8.

To determine possible reasons why database patterns did not exist for these minerals, the names and formula given for these 14 carbonate minerals in Tables A.1 through A.8 were then checked against the tabulation of accredited mineral names and formula in Mandarino and Back (2004) and the Internet online mineral database "Athena" at http://un2sg4.unige.ch/athena/mineral/mineral.html. This comparison

\footnotetext{
${ }^{1}$ The authors recognize that a crystalline solid compound formed by natural geochemical processes will likely have an accredited mineral name only if its crystallographic system and structural parameters have been determined and therefore its XRD pattern measured and/or calculated. This requirement is derived from the formal definition of a "mineral."
} 
revealed possible explanations why 13 of these 14 carbonate minerals did not have XRD database patterns. These explanations are listed as footnotes in the respective tables in this appendix.

The remaining mineral that did not show up in the search for carbonate minerals in the ICDD mineral database is dashkovaite $\left[\mathrm{Mg}\left(\mathrm{HCO}_{3}\right)_{2} \cdot 2 \mathrm{H}_{2} \mathrm{O}\right]$. A check of online sources and reference literature confirmed that these are, respectively, the accepted name and formula for this mineral as given in Table A.3. A subsequent search of the ICDD mineral database specifically for the name "dashkovaite" determined that a reference pattern existed for this mineral, but the reference pattern listed it as a "magnesium formate hydrate" with the formula $\mathrm{Mg}\left(\mathrm{HCO}_{2}\right)_{2} \cdot 2 \mathrm{H}_{2} \mathrm{O}$. Apparently the formula for dashkovaite is listed incorrectly in the ICDD database, which explains why it pattern did not show up in the initial ICDD database search for carbonate minerals.

\section{References}

Railsback LB. 1999. "Patterns in the Compositions, Properties, and Geochemistry of Carbonate Minerals." Carbonates and Evaporites 14(1):1-20.

Mandarino JA and ME Back. 2004. Fleischer's Glossary of Mineral Species 2004, The Mineralogical Record Inc., Tucson, Arizona. 
Table A.1. List of Pure Carbonate Minerals in Which Only $\mathrm{CO}_{3}{ }^{2-}$ Accounts for all the Negative Charge. Table does not include any carbonate-containing minerals tabulated by Railsback (1999), which include As, B, Ba, Bi, Cd, Ce, Co, Cr, Cu, F, Ge, Hg, La, Li, NH $, \mathrm{Nb}, \mathrm{Nd}, \mathrm{Ni}, \mathrm{Pb}$, REE, Sr, Ta U, Y Zn, and/or Zr.

\begin{tabular}{|c|c|}
\hline Mineral Name & Chemical Formula \\
\hline Ankerite & $\mathrm{C} \mathrm{CaFe}\left(\mathrm{CO}_{3}\right)_{2}$ \\
\hline Aragonite & $\mathrm{CaCO}_{3}$ \\
\hline $\begin{array}{l}\text { Breunnerite }{ }^{(a)} \text { (ferroan } \\
\text { magnesite) }\end{array}$ & $(\mathrm{Mg}, \mathrm{Fe}) \mathrm{CO}_{3}$ \\
\hline Butschliite & $\mathrm{K}_{2} \mathrm{Ca}\left(\mathrm{CO}_{3}\right)_{2}$ \\
\hline Calcite & $\mathrm{CaCO}_{3}$ \\
\hline Dolomite & $\mathrm{CaMg}\left(\mathrm{CO}_{3}\right)_{2}$ \\
\hline Eitelite & $\mathrm{Na}_{2} \mathrm{Mg}\left(\mathrm{CO}_{3}\right)_{2}$ \\
\hline Fairchildite & $\mathrm{K}_{2} \mathrm{Ca}\left(\mathrm{CO}_{3}\right)_{2}$ \\
\hline Gregoryite & $(\mathrm{Na}, \mathrm{K}, \mathrm{Ca})_{2} \mathrm{CO}_{3}$ \\
\hline Huntite & $\mathrm{Mg}_{3} \mathrm{Ca}\left(\mathrm{CO}_{3}\right)_{4}$ \\
\hline Kutnohorite & $\mathrm{CaMn}\left(\mathrm{CO}_{3}\right)_{2}$ \\
\hline Magnesite & $\mathrm{MgCO}_{3}$ \\
\hline Magniosiderite $^{(\mathrm{a})}$ & $(\mathrm{Fe}, \mathrm{Mg}) \mathrm{CO}_{3}$ \\
\hline Natrite & $\mathrm{Na}_{2} \mathrm{CO}_{3}$ \\
\hline Natrofairchildite & $\mathrm{Na}_{2} \mathrm{Ca}\left(\mathrm{CO}_{3}\right)_{2}$ \\
\hline Nyerereite & $\mathrm{Na}_{2} \mathrm{Ca}\left(\mathrm{CO}_{3}\right)_{2}$ \\
\hline $\begin{array}{l}\text { Oligonite }^{(\mathrm{a})} \\
\text { manganoan siderite }\end{array}$ & $(\mathrm{Fe}, \mathrm{Mn}) \mathrm{CO}_{3}$ \\
\hline Pistomesite $^{(a)}$ & $(\mathrm{Fe}, \mathrm{Mg}) \mathrm{CO}_{3}$ \\
\hline Rhodochrosite & $\mathrm{MnCO}_{3}$ \\
\hline Shortite & $\mathrm{Na}_{2} \mathrm{Ca}_{2}\left(\mathrm{CO}_{3}\right)_{3}$ \\
\hline Siderite & $\mathrm{FeCO}_{3}$ \\
\hline Sideroplesite ${ }^{(a)}$ & $(\mathrm{Fe}, \mathrm{Mg}) \mathrm{CO}_{3}$ \\
\hline Vaterite & $\mathrm{CaCO}_{3}$ \\
\hline Zemkorite & $\mathrm{Na}_{2} \mathrm{Ca}\left(\mathrm{CO}_{3}\right)_{2}$ \\
\hline \multicolumn{2}{|c|}{$\begin{array}{l}\text { (a) The indicated minerals are solid-solution varieties of siderite or } \\
\text { magnesite, and have mineral names that are apparently not } \\
\text { accepted. XRD patterns do not exist in the ICDD database for } \\
\text { the indicated minerals, and the mineral names are not listed in } \\
\text { Mandarino and Back (2004) or the Internet online "Athena" } \\
\text { mineral database. }\end{array}$} \\
\hline
\end{tabular}


Table A.2. List of Pure Carbonate Minerals with $\mathrm{H}_{2} \mathrm{O}$ or $\mathrm{OH}^{-}$. Table does not include any carbonatecontaining minerals tabulated by Railsback (1999), which include As, B, Ba, Bi, Cd, Ce, Co, $\mathrm{Cr}, \mathrm{Cu}, \mathrm{F}, \mathrm{Ge}, \mathrm{Hg}$, La, Li, $\mathrm{NH}_{4}, \mathrm{Nb}, \mathrm{Nd}, \mathrm{Ni}, \mathrm{Pb}, \mathrm{REE}, \mathrm{Sr}, \mathrm{Ta} \mathrm{U}, \mathrm{Y} \mathrm{Zn}$, and/or Zr.

\begin{tabular}{|c|c|}
\hline Mineral Name & Chemical Formula \\
\hline Barringtonite & $\mathrm{MgCO}_{3} \cdot 2 \mathrm{H}_{2} \mathrm{O}$ \\
\hline Baylissite & $\mathrm{K}_{2} \mathrm{Mg}\left(\mathrm{CO}_{3}\right)_{2} \cdot 4 \mathrm{H}_{2} \mathrm{O}$ \\
\hline Dawsonite & $\mathrm{NaAlCO}_{3}(\mathrm{OH})_{2}$ \\
\hline Gaylussite & $\mathrm{Na}_{2} \mathrm{Ca}\left(\mathrm{CO}_{3}\right)_{2} \cdot 5 \mathrm{H}_{2} \mathrm{O}$ \\
\hline Ikaite & $\mathrm{CaCO}_{3} \cdot 6 \mathrm{H}_{2} \mathrm{O}$ \\
\hline Lansfordite & $\mathrm{MgCO}_{3} \cdot 5 \mathrm{H}_{2} \mathrm{O}$ \\
\hline Monohydrocalcite & $\mathrm{CaCO}_{3} \cdot \mathrm{H}_{2} \mathrm{O}$ \\
\hline Natron & $\mathrm{Na}_{2} \mathrm{CO}_{3} \cdot 10 \mathrm{H}_{2} \mathrm{O}$ \\
\hline Nesquehonite & $\mathrm{MgCO}_{3} \cdot 3 \mathrm{H}_{2} \mathrm{O}$ \\
\hline Pirssonite & $\mathrm{Na}_{2} \mathrm{Ca}\left(\mathrm{CO}_{3}\right)_{2} \cdot 2 \mathrm{H}_{2} \mathrm{O}$ \\
\hline Sergeevite & $\mathrm{Ca}_{2} \mathrm{Mg}_{11}\left(\mathrm{CO}_{3}\right)_{13} \cdot 10 \mathrm{H}_{2} \mathrm{O}$ \\
\hline Thermonatrite & $\mathrm{Na}_{2} \mathrm{CO}_{3} \cdot \mathrm{H}_{2} \mathrm{O}$ \\
\hline
\end{tabular}

Table A.3. List of Pure Carbonate Minerals in Which Only $\mathrm{HCO}_{3}{ }^{-}$Account for all the Negative Charge. Table does not include any carbonate-containing minerals tabulated by Railsback (1999), which include As, B, Ba, Bi, Cd, Ce, Co, Cr, Cu, F, Ge, Hg, La, Li, NH $4, \mathrm{Nb}, \mathrm{Nd}, \mathrm{Ni}, \mathrm{Pb}$, REE, Sr, Ta U, Y Zn, and/or Zr.

\begin{tabular}{|l|l||}
\hline \multicolumn{1}{|c|}{ Mineral Name } & \multicolumn{1}{c|}{ Bicarbonates } \\
\hline \hline \multicolumn{2}{|c||}{ Chemical Formula } \\
\hline Dashkovaite ${ }^{(\mathrm{a})}$ & $\mathrm{Mg}\left(\mathrm{HCO}_{3}\right)_{2} \cdot 2 \mathrm{H}_{2} \mathrm{O}$ \\
\hline Kalicinite & $\mathrm{KHCO}_{3}$ \\
\hline Nahcolite & $\mathrm{NaHCO}_{3}$ \\
\hline Trona & $\mathrm{Na}_{3} \mathrm{HCO}_{3} \mathrm{CO}_{3} \cdot 2 \mathrm{H}_{2} \mathrm{O}$ \\
\hline Wegscheiderite & $\mathrm{Na}_{5}\left(\mathrm{HCO}_{3}\right)_{3} \mathrm{CO}_{3}$ \\
\hline $\begin{array}{l}\text { (a) It is not known why there is no XRD pattern for dashkovaite in the ICDD } \\
\text { database. The mineral name and formula listed above are identical to those in } \\
\text { Mandarino and Back (2004) and the Internet online "Athena" mineral database. }\end{array}$ \\
\hline
\end{tabular}


Table A.4. List of Pure Carbonates with $\mathrm{H}_{2} \mathrm{O}$ and $\mathrm{OH}^{-}$, and Layered Hydroxyl-Carbonate Minerals. Table does not include any carbonate-containing minerals tabulated by Railsback (1999), which include As, B, Ba, Bi, Cd, Ce, Co, Cr, Cu, F, Ge, Hg, La, Li, $\mathrm{NH}_{4}, \mathrm{Nb}, \mathrm{Nd}, \mathrm{Ni}, \mathrm{Pb}$, REE, Sr, Ta U, Y Zn, and/or Zr.

\begin{tabular}{|l|l||}
\hline \multicolumn{1}{|c|}{ Mineral Name } & \multicolumn{1}{c|}{ Chemical Formula } \\
\hline Alumohydrocalcite & $\mathrm{CaAl}_{2}\left(\mathrm{CO}_{3}\right)_{2}(\mathrm{OH})_{4} \cdot 3 \mathrm{H}_{2} \mathrm{O}$ \\
\hline Artinite & $\mathrm{Mg}_{2} \mathrm{CO}_{3}(\mathrm{OH})_{2} \cdot 3 \mathrm{H}_{2} \mathrm{O}$ \\
\hline Brugnatellite & $\mathrm{Mg}_{6} \mathrm{Fe}^{3+} \mathrm{CO}_{3}(\mathrm{OH})_{13} \cdot 4 \mathrm{H}_{2} \mathrm{O}$ \\
\hline Caresite & $\mathrm{Fe}_{4} \mathrm{Al}_{2}(\mathrm{OH})_{12} \mathrm{CO}_{3} \cdot 3 \mathrm{H}_{2} \mathrm{O}$ \\
\hline Charmarite & $\mathrm{Mn}_{4} \mathrm{Al}_{2}(\mathrm{OH})_{12} \mathrm{CO}_{3} \cdot 3 \mathrm{H}_{2} \mathrm{O}$ \\
\hline Coalingite & $\mathrm{Mg}_{10} \mathrm{Fe}^{3+}{ }_{2} \mathrm{CO}_{3}(\mathrm{OH})_{24} \cdot 2 \mathrm{H}_{2} \mathrm{O}$ \\
\hline Desautelsite & $\mathrm{Mg}_{6} \mathrm{Mn}^{3+}{ }_{2} \mathrm{CO}_{3}(\mathrm{OH})_{16} \cdot 4 \mathrm{H}_{2} \mathrm{O}$ \\
\hline Dypingite & $\mathrm{Mg}_{5}\left(\mathrm{CO}_{3}\right)_{4}(\mathrm{OH})_{2} \cdot 5 \mathrm{H}_{2} \mathrm{O}$ \\
\hline Giorgiosite & $\mathrm{Mg}_{5}\left(\mathrm{CO}_{3}\right)_{4}(\mathrm{OH})_{2} \cdot 4 \mathrm{H}_{2} \mathrm{O}$ \\
\hline Hydromagnesite & $\mathrm{Mg}_{5}\left(\mathrm{CO}_{3}\right)_{4}(\mathrm{OH})_{2} \cdot 4 \mathrm{H}_{2} \mathrm{O}$ \\
\hline Hydroscarbroite & $\mathrm{Al}_{14}\left(\mathrm{CO}_{3}\right)_{3}(\mathrm{OH})_{36} \cdot \mathrm{nH}_{2} \mathrm{O}$ \\
\hline Hydrotalcite & $\mathrm{Mg}_{6} \mathrm{Al}_{2} \mathrm{CO}_{3}(\mathrm{OH})_{16} \cdot 4 \mathrm{H}_{2} \mathrm{O}$ \\
\hline Indigirite & $\mathrm{Mg}_{2} \mathrm{Al}_{2}(\mathrm{CO})_{4}(\mathrm{OH})_{2} \cdot 15 \mathrm{H}_{2} \mathrm{O}$ \\
\hline Manasseite & $\mathrm{Mg}_{6} \mathrm{Al}_{2} \mathrm{CO}_{3}(\mathrm{OH})_{16} \cdot 4 \mathrm{H}_{2} \mathrm{O}$ \\
\hline Para-alumohydrocalcite & $\mathrm{CaAl}_{2}\left(\mathrm{CO}_{3}\right)_{2}(\mathrm{OH})_{4} \cdot 6 \mathrm{H}_{2} \mathrm{O}$ \\
\hline Pokrovskite & $\mathrm{Mg}_{2} \mathrm{CO}_{3}(\mathrm{OH})_{2} \cdot 0.5 \mathrm{H}_{2} \mathrm{O}$ \\
\hline Pyroaurite & $\mathrm{Mg}_{6} \mathrm{Fe}^{3+}{ }_{2} \mathrm{CO}_{3}(\mathrm{OH})_{16} \cdot 4 \mathrm{H}_{2} \mathrm{O}$ \\
\hline Quintinite & $\mathrm{Mg}_{4} \mathrm{Al}_{2}(\mathrm{OH})_{12} \mathrm{CO}{ }_{3} \cdot 3 \mathrm{H}_{2} \mathrm{O}$ \\
\hline Scarbroite & $\mathrm{Al}_{5} \mathrm{CO}_{3}(\mathrm{OH})_{13} \cdot 5 \mathrm{H}_{2} \mathrm{O}$ \\
\hline Sjogrenite & $\mathrm{Mg}_{6} \mathrm{Fe}^{3+}{ }_{2} \mathrm{CO}_{3}(\mathrm{OH})_{16} \cdot 4 \mathrm{H}_{2} \mathrm{O}$ \\
\hline Wermlandite & $\mathrm{Ca}_{2} \mathrm{Mg}_{14}\left(\mathrm{Fe}{ }^{3+}, \mathrm{Al}\right)_{4} \mathrm{CO}_{3}(\mathrm{OH})_{42} \cdot 29 \mathrm{H}_{2} \mathrm{O}$ \\
\hline Yoshikawaite & $\mathrm{Mg}_{5}\left(\mathrm{CO}_{3}\right)_{4}(\mathrm{OH})_{2} \cdot 8 \mathrm{H}_{2} \mathrm{O}$ \\
\hline (a) The indicated mineral may correspond to dypingite $(\mathrm{see}$ above in same table). There is no XRD \\
\hline
\end{tabular}


Table A.5. List of Chloride-Containing Carbonates. Table does not include any carbonate-containing minerals tabulated by Railsback (1999), which include As, B, Ba, Bi, Cd, Ce, Co, Cr, Cu, F, $\mathrm{Ge}, \mathrm{Hg}$, La, Li, $\mathrm{NH}_{4}, \mathrm{Nb}, \mathrm{Nd}, \mathrm{Ni}, \mathrm{Pb}, \mathrm{REE}, \mathrm{Sr}, \mathrm{Ta} \mathrm{U}, \mathrm{Y} \mathrm{Zn}$, and/or Zr.

\begin{tabular}{|c|c|}
\hline Mineral Name & Chemical Formula \\
\hline Afghanite $^{(a)}$ & $\left(\mathrm{N}(\mathrm{Na}, \mathrm{Ca}, \mathrm{K})_{8}(\mathrm{Si}, \mathrm{Al})_{12} \mathrm{O}_{24}\left(\mathrm{SO}_{4}, \mathrm{Cl}, \mathrm{CO}_{3}\right)_{3} \cdot \mathrm{H}_{2} \mathrm{O}\right.$ \\
\hline Chlorartinite & $\mathrm{Mg}_{2}\left(\mathrm{CO}_{3}\right) \mathrm{Cl}(\mathrm{OH}) \cdot 3\left(\mathrm{H}_{2} \mathrm{O}\right)$ \\
\hline Chlormagaluminite & $(\mathrm{MgFe})_{4} \mathrm{Al}_{2}(\mathrm{OH})_{12}\left(\mathrm{Cl}_{2}, \mathrm{CO}_{3}\right) \cdot 2 \mathrm{H}_{2} \mathrm{O}$ \\
\hline Davyne & $(\mathrm{Na}, \mathrm{Ca}, \mathrm{K})_{8} \mathrm{Al}_{6} \mathrm{Si}_{6} \mathrm{O}_{24}\left(\mathrm{Cl}, \mathrm{SO}_{4}, \mathrm{CO}_{3}\right)_{2-3}$ \\
\hline Defernite & $\mathrm{Ca}_{3} \mathrm{CO}_{3}(\mathrm{OH}, \mathrm{Cl})_{4} \cdot \mathrm{H}_{2} \mathrm{O}$ \\
\hline Franzinite $^{(\mathrm{a})}$ & $(\mathrm{Na}, \mathrm{Ca})_{7}(\mathrm{Si}, \mathrm{Al})_{12} \mathrm{O}_{24}\left(\mathrm{SO}_{4}, \mathrm{CO}_{3}, \mathrm{OH}, \mathrm{Cl}\right)_{3} \cdot \mathrm{H}_{2} \mathrm{O}$ \\
\hline Hanksite & $\mathrm{Na}_{22} \mathrm{~K}\left(\mathrm{SO}_{4}\right)_{9}\left(\mathrm{CO}_{3}\right)_{2} \mathrm{Cl}$ \\
\hline Holdawayite & $\mathrm{Mn}_{6}\left(\mathrm{CO}_{3}\right)_{2}(\mathrm{OH})_{7}(\mathrm{Cl}, \mathrm{OH})$ \\
\hline Liottite $^{(a)}$ & $(\mathrm{Ca}, \mathrm{Na})_{4}(\mathrm{Si}, \mathrm{Al})_{6} \mathrm{O}_{12}\left(\mathrm{SO}_{4}, \mathrm{CO}_{3}, \mathrm{OH}, \mathrm{Cl}\right)_{2} \cdot \mathrm{nH}_{2} \mathrm{O}$ \\
\hline Microsommite $^{(\mathrm{a})}$ & $(\mathrm{Na}, \mathrm{Ca}, \mathrm{K})_{7-8}(\mathrm{Si}, \mathrm{Al})_{12} \mathrm{O}_{24}\left(\mathrm{Cl}, \mathrm{SO}_{4}, \mathrm{CO}_{3}\right)_{2-3}$ \\
\hline Northupite & $\mathrm{Na}_{3} \mathrm{Mg}\left(\mathrm{CO}_{3}\right)_{2} \mathrm{Cl}$ \\
\hline Sacrofanite $^{(a)}$ & $(\mathrm{Na}, \mathrm{Ca}, \mathrm{K})_{9}(\mathrm{Si}, \mathrm{Al})_{12} \mathrm{O}_{24}\left[(\mathrm{OH})_{2}, \mathrm{SO}_{4}, \mathrm{CO}_{3}, \mathrm{Cl}_{2}\right]_{3} \cdot \mathrm{nH}_{2} \mathrm{O}$ \\
\hline Sheldrickite & $\mathrm{NaCa}_{3}\left(\mathrm{CO}_{3}\right)_{2} \mathrm{~F}_{3} \cdot \mathrm{H}_{2} \mathrm{O}$ \\
\hline Tatarskite & $\mathrm{Ca}_{6} \mathrm{Mg}_{2}\left(\mathrm{SO}_{4}\right)_{2}\left(\mathrm{CO}_{3}\right)_{2} \mathrm{Cl}_{4}(\mathrm{OH})_{4} \cdot 7 \mathrm{H}_{2} \mathrm{O}$ \\
\hline Tunisite & $\mathrm{NaCa}_{2} \mathrm{Al}_{4}\left(\mathrm{CO}_{3}\right)_{4}(\mathrm{OH})_{8} \mathrm{Cl}$ \\
\hline Vishnevite $^{(\mathrm{a})}$ & $(\mathrm{Na}, \mathrm{Ca}, \mathrm{K})_{6}(\mathrm{Si}, \mathrm{Al})_{12} \mathrm{O}_{24}\left(\mathrm{SO}_{4}, \mathrm{CO}_{3}, \mathrm{Cl}_{2}\right)_{2-4} \cdot \mathrm{nH}_{2} \mathrm{O}$ \\
\hline \multicolumn{2}{|c|}{$\begin{array}{l}\text { (a) The formula formally accepted for the noted minerals do not contain } \mathrm{CO}_{3} \text {. All of the noted } \\
\text { minerals are members of the cancrinite mineral group that have the general formula } \\
\mathrm{A}_{6-9}(\mathrm{Si}, \mathrm{Al})_{12} \mathrm{O}_{24}\left[\left(\mathrm{SO}_{4}\right),\left(\mathrm{CO}_{3}\right), \mathrm{Cl}_{2},(\mathrm{OH})_{2}\right]_{2-4} \cdot \mathrm{nH}_{2} \mathrm{O} \text { where } \mathrm{A} \text { is Na, } \mathrm{Ca} \text {, and/or } \mathrm{K} \text {. The noted } \\
\text { mineral names are listed in Mandarino and } \mathrm{Back}(2004) \text { and the Internet online "Athena" } \\
\text { mineral database, but the formula listed in those sources do not include } \mathrm{CO}_{3} \text { as given } \\
\text { above. XRD patterns for the indicated minerals do not exist in the ICDD database but the } \\
\text { comparison was based on a search of only the carbonate-containing minerals in the ICDD } \\
\text { database. }\end{array}$} \\
\hline
\end{tabular}


Table A.6. List of Sulfate-Containing Carbonates. Table does not include any carbonate-containing minerals tabulated by Railsback (1999), which include As, B, Ba, Bi, Cd, Ce, Co, Cr, Cu, F, $\mathrm{Ge}, \mathrm{Hg}$, La, Li, $\mathrm{NH}_{4}, \mathrm{Nb}, \mathrm{Nd}, \mathrm{Ni}, \mathrm{Pb}, \mathrm{REE}, \mathrm{Sr}, \mathrm{Ta} \mathrm{U}, \mathrm{Y} \mathrm{Zn}$, and/or Zr.

\begin{tabular}{|c|c|}
\hline Mineral Name & Chemical Formula \\
\hline Afghanite $^{(a)}$ & $\left(\mathrm{N}(\mathrm{Na}, \mathrm{Ca}, \mathrm{K})_{8}(\mathrm{Si}, \mathrm{Al})_{12} \mathrm{O}_{24}\left(\mathrm{SO}_{4}, \mathrm{Cl}, \mathrm{CO}_{3}\right)_{3} \cdot \mathrm{H}_{2} \mathrm{O}\right.$ \\
\hline Burkeite & $\mathrm{Na}_{6} \mathrm{CO}_{3}\left(\mathrm{SO}_{4}\right)_{2}$ \\
\hline Davyne & $(\mathrm{Na}, \mathrm{Ca}, \mathrm{K})_{8} \mathrm{Al}_{6} \mathrm{Si}_{6} \mathrm{O}_{24}\left(\mathrm{Cl}, \mathrm{SO}_{4}, \mathrm{CO}_{3}\right)_{2-3}$ \\
\hline Ferrotychite & $\mathrm{Na}_{6}(\mathrm{Fe}, \mathrm{Mn}, \mathrm{Mg})_{2} \mathrm{SO}_{4}\left(\mathrm{CO}_{3}\right)_{4}$ \\
\hline Franzinite $^{(\mathrm{a})}$ & $(\mathrm{Na}, \mathrm{Ca})_{7}(\mathrm{Si}, \mathrm{Al})_{12} \mathrm{O}_{24}\left(\mathrm{SO}_{4}, \mathrm{CO}_{3}, \mathrm{OH}, \mathrm{Cl}\right)_{3} \cdot \mathrm{H}_{2} \mathrm{O}$ \\
\hline Hanksite & $\mathrm{Na}_{22} \mathrm{~K}\left(\mathrm{SO}_{4}\right)_{9}\left(\mathrm{CO}_{3}\right)_{2} \mathrm{Cl}$ \\
\hline Jouravskite & $\mathrm{Ca}_{3} \mathrm{Mn}^{4+}\left(\mathrm{SO}_{4}, \mathrm{CO}_{3}\right)_{2}(\mathrm{OH})_{6} \cdot 13 \mathrm{H}_{2} \mathrm{O}$ \\
\hline Latiumite & $\mathrm{K}_{2} \mathrm{Ca}_{6}(\mathrm{Si}, \mathrm{Al})_{11} \mathrm{O}_{25}\left(\mathrm{SO}_{4}, \mathrm{CO}_{3}\right)$ \\
\hline Liottite $^{(\mathrm{a})}$ & $(\mathrm{Ca}, \mathrm{Na})_{4}(\mathrm{Si}, \mathrm{Al})_{6} \mathrm{O}_{12}\left(\mathrm{SO}_{4}, \mathrm{CO}_{3}, \mathrm{OH}, \mathrm{Cl}\right)_{2} \cdot \mathrm{nH}_{2} \mathrm{O}$ \\
\hline Manganotychite & $\mathrm{Na}_{6} \mathrm{Mn}_{2} \mathrm{SO}_{4}\left(\mathrm{CO}_{3}\right)_{4}$ \\
\hline Microsommite $^{(\mathrm{a})}$ & $(\mathrm{Na}, \mathrm{Ca}, \mathrm{K})_{7-8}(\mathrm{Si}, \mathrm{Al})_{12} \mathrm{O}_{24}\left(\mathrm{Cl}, \mathrm{SO}_{4}, \mathrm{CO}_{3}\right)_{2-3}$ \\
\hline Motukoreaite & $\mathrm{NaMg}_{19} \mathrm{Al}_{12}\left(\mathrm{CO}_{3}\right)_{6.5}\left(\mathrm{SO}_{4}\right)_{4}(\mathrm{OH})_{54} \cdot 28 \mathrm{H}_{2} \mathrm{O}$ \\
\hline Rapidcreekite & $\mathrm{Ca}_{2} \mathrm{SO}_{4} \mathrm{CO}_{3} \cdot 4 \mathrm{H}_{2} \mathrm{O}$ \\
\hline Sacrofanite $^{(a)}$ & $(\mathrm{Na}, \mathrm{Ca}, \mathrm{K})_{9}(\mathrm{Si}, \mathrm{Al})_{12} \mathrm{O}_{24}\left[(\mathrm{OH})_{2}, \mathrm{SO}_{4}, \mathrm{CO}_{3}, \mathrm{Cl}_{2}\right]_{3} \cdot \mathrm{nH}_{2} \mathrm{O}$ \\
\hline Silvialite & $\mathrm{Ca}_{4} \mathrm{Al}_{6} \mathrm{Si}_{6} \mathrm{O}_{24}\left(\mathrm{SO}_{4}\right)_{0.6}\left(\mathrm{CO}_{3}\right)_{0.4}$ \\
\hline Tatarskite & $\mathrm{Ca}_{6} \mathrm{Mg}_{2}\left(\mathrm{SO}_{4}\right)_{2}\left(\mathrm{CO}_{3}\right)_{2} \mathrm{Cl}_{4}(\mathrm{OH})_{4} \cdot 7 \mathrm{H}_{2} \mathrm{O}$ \\
\hline Thaumasite & $\mathrm{Ca}_{3} \mathrm{SiCO}_{3} \mathrm{SO}_{4}(\mathrm{OH})_{6} \cdot 12 \mathrm{H}_{2} \mathrm{O}$ \\
\hline Tuscanite & $\mathrm{K}(\mathrm{Ca}, \mathrm{Na})_{6}(\mathrm{Si}, \mathrm{Al})_{10} \mathrm{O}_{22}\left[\mathrm{SO}_{4}, \mathrm{CO}_{3},(\mathrm{OH})_{2}\right] \cdot \mathrm{H}_{2} \mathrm{O}$ \\
\hline Tychite & $\mathrm{Na}_{6} \mathrm{Mg}_{2} \mathrm{SO}_{4}\left(\mathrm{CO}_{3}\right)_{4}$ \\
\hline Vishnevite $^{(a)}$ & $(\mathrm{Na}, \mathrm{Ca}, \mathrm{K})_{6}(\mathrm{Si}, \mathrm{Al})_{12} \mathrm{O}_{24}\left(\mathrm{SO}_{4}, \mathrm{CO}_{3}, \mathrm{Cl}_{2}\right)_{2-4} \cdot \mathrm{nH}_{2} \mathrm{O}$ \\
\hline \multicolumn{2}{|c|}{$\begin{array}{l}\text { (a) The formula formally accepted for the noted minerals do not contain } \mathrm{CO}_{3} \text {. All of the noted } \\
\text { minerals are members of the cancrinite mineral group that have the general formula } \\
\mathrm{A}_{6-9}(\mathrm{Si}, \mathrm{Al})_{12} \mathrm{O}_{24}\left[\left(\mathrm{SO}_{4}\right),\left(\mathrm{CO}_{3}\right), \mathrm{Cl}_{2},(\mathrm{OH})_{2}\right]_{2-4} \cdot \mathrm{nH}_{2} \mathrm{O} \text { where } \mathrm{A} \text { is Na, } \mathrm{Ca} \text {, and/or } \mathrm{K} \text {. The noted } \\
\text { mineral names are listed in Mandarino and } \mathrm{Back}(2004) \text { and the Internet online "Athena" } \\
\text { mineral database, but the formula listed in those sources do not include } \mathrm{CO}_{3} \text { as given above. } \\
\text { XRD patterns for the indicated minerals do not exist in the ICDD database but the comparison } \\
\text { was based on a search of only the carbonate-containing minerals in the ICDD database. }\end{array}$} \\
\hline
\end{tabular}


Table A.7. List of Silicate-Containing Carbonates. Table does not include any carbonate-containing minerals tabulated by Railsback (1999), which include As, B, Ba, Bi, Cd, Ce, Co, Cr, Cu, F, $\mathrm{Ge}, \mathrm{Hg}$, La, Li, $\mathrm{NH}_{4}, \mathrm{Nb}, \mathrm{Nd}, \mathrm{Ni}, \mathrm{Pb}, \mathrm{REE}, \mathrm{Sr}, \mathrm{Ta} \mathrm{U}, \mathrm{Y} \mathrm{Zn}$, and/or Zr.

\begin{tabular}{|c|c|}
\hline Mineral Name & Chemical Formula \\
\hline Afghanite $^{(a)}$ & $\left(\mathrm{N}(\mathrm{Na}, \mathrm{Ca}, \mathrm{K})_{8}(\mathrm{Si}, \mathrm{Al})_{12} \mathrm{O}_{24}\left(\mathrm{SO}_{4}, \mathrm{Cl}_{1} \mathrm{CO}_{3}\right)_{3} \cdot \mathrm{H}_{2} \mathrm{O}\right.$ \\
\hline Cancrinite & $\mathrm{Na}_{6} \mathrm{Ca}_{2} \mathrm{Al}_{6} \mathrm{Si}_{6} \mathrm{O}_{24}\left(\mathrm{CO}_{3}\right)_{2}$ \\
\hline Cancrisilite & $\mathrm{Na}_{7}\left(\mathrm{Al}_{5} \mathrm{Si}_{7} \mathrm{O}_{24}\right) \mathrm{CO}_{3} \cdot 3 \mathrm{H}_{2} \mathrm{O}$ \\
\hline Carletonite & $\mathrm{KNa}_{4} \mathrm{Ca}_{4} \mathrm{Si}_{8} \mathrm{O}_{18}\left(\mathrm{CO}_{3}\right)_{4}(\mathrm{OH}, \mathrm{F}) \cdot \mathrm{H}_{2} \mathrm{O}$ \\
\hline Davyne & $(\mathrm{Na}, \mathrm{Ca}, \mathrm{K})_{8} \mathrm{Al}_{6} \mathrm{Si}_{6} \mathrm{O}_{24}\left(\mathrm{Cl}, \mathrm{SO}_{4}, \mathrm{CO}_{3}\right)_{2-3}$ \\
\hline Franzinite $^{(a)}$ & $(\mathrm{Na}, \mathrm{Ca})_{7}(\mathrm{Si}, \mathrm{Al})_{12} \mathrm{O}_{24}\left(\mathrm{SO}_{4}, \mathrm{CO}_{3}, \mathrm{OH}, \mathrm{Cl}\right)_{3} \cdot \mathrm{H}_{2} \mathrm{O}$ \\
\hline Fukalite & $\mathrm{Ca}_{4} \mathrm{Si}_{2} \mathrm{O}_{6} \mathrm{CO}_{3}(\mathrm{OH}, \mathrm{F})_{2}$ \\
\hline Hydroxycancrinite $^{(a)}$ & $\mathrm{Na}_{8}\left(\mathrm{Al}_{6} \mathrm{Si}_{6} \mathrm{O}_{24}\right)\left(\mathrm{OH}, \mathrm{CO}_{3}\right)_{2} \cdot 2 \mathrm{H}_{2} \mathrm{O}$ \\
\hline Latiumite & $\mathrm{K}_{2} \mathrm{Ca}_{6}(\mathrm{Si}, \mathrm{Al})_{11} \mathrm{O}_{25}\left(\mathrm{SO}_{4}, \mathrm{CO}_{3}\right)$ \\
\hline Liottite $^{(a)}$ & $(\mathrm{Ca}, \mathrm{Na})_{4}(\mathrm{Si}, \mathrm{Al})_{6} \mathrm{O}_{12}\left(\mathrm{SO}_{4}, \mathrm{CO}_{3}, \mathrm{OH}, \mathrm{Cl}\right)_{2} \cdot \mathrm{nH}_{2} \mathrm{O}$ \\
\hline Meionite & $\mathrm{Ca}_{4} \mathrm{Al}_{6} \mathrm{Si}_{6} \mathrm{O}_{24} \mathrm{CO}_{3}$ \\
\hline Microsommite $^{(\mathrm{a})}$ & $(\mathrm{Na}, \mathrm{Ca}, \mathrm{K})_{7-8}(\mathrm{Si}, \mathrm{Al})_{12} \mathrm{O}_{24}\left(\mathrm{Cl}, \mathrm{SO}_{4}, \mathrm{CO}_{3}\right)_{2-3}$ \\
\hline Paraspurrite & $\mathrm{Ca}_{5} \mathrm{Si}_{2} \mathrm{O}_{8} \mathrm{CO}_{3}$ \\
\hline Sacrofanite $^{(a)}$ & $(\mathrm{Na}, \mathrm{Ca}, \mathrm{K})_{9}(\mathrm{Si}, \mathrm{Al})_{12} \mathrm{O}_{24}\left[(\mathrm{OH})_{2}, \mathrm{SO}_{4}, \mathrm{CO}_{3}, \mathrm{Cl}_{2}\right]_{3} \cdot \mathrm{nH}_{2} \mathrm{O}$ \\
\hline Scawtite & $\mathrm{Ca}_{7} \mathrm{Si}_{6} \mathrm{O}_{18} \mathrm{CO}_{3} \cdot 2 \mathrm{H}_{2} \mathrm{O}$ \\
\hline Silvialite & $\mathrm{Ca}_{4} \mathrm{Al}_{6} \mathrm{Si}_{6} \mathrm{O}_{24}\left(\mathrm{SO}_{4}\right)_{0.6}\left(\mathrm{CO}_{3}\right)_{0.4}$ \\
\hline Spurrite & $\mathrm{Ca}_{5} \mathrm{Si}_{2} \mathrm{O}_{8} \mathrm{CO}_{3}$ \\
\hline Thaumasite & $\mathrm{Ca}_{3} \mathrm{SiCO}_{3} \mathrm{SO}_{4}(\mathrm{OH})_{6} \cdot 12 \mathrm{H}_{2} \mathrm{O}$ \\
\hline Tilleyite & $\mathrm{Ca}_{5} \mathrm{Si}_{2} \mathrm{O}_{7}\left(\mathrm{CO}_{3}\right)_{2}$ \\
\hline Tuscanite & $\mathrm{K}(\mathrm{Ca}, \mathrm{Na})_{6}(\mathrm{Si}, \mathrm{Al})_{10} \mathrm{O}_{22}\left[\mathrm{SO}_{4}, \mathrm{CO}_{3},(\mathrm{OH})_{2}\right] \cdot \mathrm{H}_{2} \mathrm{O}$ \\
\hline Vishnevite $^{(a)}$ & $(\mathrm{Na}, \mathrm{Ca}, \mathrm{K})_{6}(\mathrm{Si}, \mathrm{Al})_{12} \mathrm{O}_{24}\left(\mathrm{SO}_{4}, \mathrm{CO}_{3}, \mathrm{Cl}_{2}\right)_{2-4} \cdot \mathrm{nH}_{2} \mathrm{O}$ \\
\hline \multicolumn{2}{|c|}{$\begin{array}{l}\text { (a) The formula formally accepted for the noted minerals do not contain } \mathrm{CO}_{3} \text {. All of the } \\
\text { noted minerals are members of the cancrinite mineral group that have the general formula } \\
\mathrm{A}_{6-9}(\mathrm{Si}, \mathrm{Al})_{12} \mathrm{O}_{24}\left[\left(\mathrm{SO}_{4}\right),\left(\mathrm{CO}_{3}\right), \mathrm{Cl}_{2},(\mathrm{OH})_{2}\right]_{2-4} \cdot \mathrm{nH}_{2} \mathrm{O} \text { where } \mathrm{A} \text { is Na, } \mathrm{Ca} \text {, and/or } \mathrm{K} \text {. The noted } \\
\text { mineral names are listed in Mandarino and } \mathrm{Back}(2004) \text { and the Internet online "Athena" } \\
\text { mineral database, but the formula listed in those sources do not include } \mathrm{CO}_{3} \text { as given } \\
\text { above. XRD patterns for the indicated minerals do not exist in the ICDD database but the } \\
\text { comparison was based on a search of only the carbonate-containing minerals in the ICDD } \\
\text { database. }\end{array}$} \\
\hline
\end{tabular}


Table A.8. List of Carbonates that Contain Two or More Other Anions other than $\mathrm{CO}_{3}{ }^{2-}$ or $\mathrm{HCO}_{3}{ }^{-}$. Table does not include any carbonate-containing minerals tabulated by Railsback (1999), which include As, B, Ba, Bi, Cd, Ce, Co, Cr, Cu, F, Ge, Hg, La, Li, NH${ }_{4}, \mathrm{Nb}, \mathrm{Nd}, \mathrm{Ni}, \mathrm{Pb}$, REE, Sr, Ta U, Y Zn, and/or Zr.

\begin{tabular}{|c|c|}
\hline Mineral Name & Chemical Formula \\
\hline Afghanite $^{(\mathrm{a})}$ & $(\mathrm{Na}, \mathrm{Ca}, \mathrm{K})_{8}(\mathrm{Si}, \mathrm{Al})_{12} \mathrm{O}_{24}\left(\mathrm{SO}_{4}, \mathrm{Cl}, \mathrm{CO}_{3}\right)_{3} \cdot \mathrm{H}_{2} \mathrm{O}$ \\
\hline Davyne & $(\mathrm{Na}, \mathrm{Ca}, \mathrm{K})_{8} \mathrm{Al}_{6} \mathrm{Si}_{6} \mathrm{O}_{24}\left(\mathrm{Cl}, \mathrm{SO}_{4}, \mathrm{CO}_{3}\right)_{2-3}$ \\
\hline Franzinite $^{(a)}$ & $(\mathrm{Na}, \mathrm{Ca})_{7}(\mathrm{Si}, \mathrm{Al})_{12} \mathrm{O}_{24}\left(\mathrm{SO}_{4}, \mathrm{CO}_{3}, \mathrm{OH}, \mathrm{Cl}\right)_{3} \cdot \mathrm{H}_{2} \mathrm{O}$ \\
\hline Hanksite & $\mathrm{Na}_{22} \mathrm{~K}\left(\mathrm{SO}_{4}\right)_{9}\left(\mathrm{CO}_{3}\right)_{2} \mathrm{Cl}$ \\
\hline Latiumite & $\mathrm{K}_{2} \mathrm{Ca}_{6}(\mathrm{Si}, \mathrm{Al})_{11} \mathrm{O}_{25}\left(\mathrm{SO}_{4}, \mathrm{CO}_{3}\right)$ \\
\hline Liottite $^{(a)}$ & $(\mathrm{Ca}, \mathrm{Na})_{4}(\mathrm{Si}, \mathrm{Al})_{6} \mathrm{O}_{12}\left(\mathrm{SO}_{4}, \mathrm{CO}_{3}, \mathrm{OH}, \mathrm{Cl}\right)_{2} \cdot \mathrm{nH}_{2} \mathrm{O}$ \\
\hline Microsommite $^{(a)}$ & $(\mathrm{Na}, \mathrm{Ca}, \mathrm{K})_{7-8}(\mathrm{Si}, \mathrm{Al})_{12} \mathrm{O}_{24}\left(\mathrm{Cl}, \mathrm{SO}_{4}, \mathrm{CO}_{3}\right)_{2-3}$ \\
\hline Sacrofanite $^{(\mathrm{a})}$ & $(\mathrm{Na}, \mathrm{Ca}, \mathrm{K})_{9}(\mathrm{Si}, \mathrm{Al})_{12} \mathrm{O}_{24}\left[(\mathrm{OH})_{2}, \mathrm{SO}_{4}, \mathrm{CO}_{3}, \mathrm{Cl}_{2}\right]_{3} \cdot \mathrm{nH}_{2} \mathrm{O}$ \\
\hline Silvialite & $\mathrm{Ca}_{4} \mathrm{Al}_{6} \mathrm{Si}_{6} \mathrm{O}_{24}\left(\mathrm{SO}_{4}\right)_{0.6}\left(\mathrm{CO}_{3}\right)_{0.4}$ \\
\hline Tatarskite & $\mathrm{Ca}_{6} \mathrm{Mg}_{2}\left(\mathrm{SO}_{4}\right)_{2}\left(\mathrm{CO}_{3}\right)_{2} \mathrm{Cl}_{4}(\mathrm{OH})_{4} \cdot 7 \mathrm{H}_{2} \mathrm{O}$ \\
\hline Thaumasite & $\mathrm{Ca}_{3} \mathrm{SiCO}_{3} \mathrm{SO}_{4}(\mathrm{OH})_{6} \cdot 12 \mathrm{H}_{2} \mathrm{O}$ \\
\hline Tuscanite & $\mathrm{K}(\mathrm{Ca}, \mathrm{Na})_{6}(\mathrm{Si}, \mathrm{Al})_{10} \mathrm{O}_{22}\left[\mathrm{SO}_{4}, \mathrm{CO}_{3},(\mathrm{OH})_{2}\right] \cdot \mathrm{H}_{2} \mathrm{O}$ \\
\hline Vishnevite $^{(a)}$ & $(\mathrm{Na}, \mathrm{Ca}, \mathrm{K})_{6}(\mathrm{Si}, \mathrm{Al})_{12} \mathrm{O}_{24}\left(\mathrm{SO}_{4}, \mathrm{CO}_{3}, \mathrm{Cl}_{2}\right)_{2-4} \cdot \mathrm{nH}_{2} \mathrm{O}$ \\
\hline \multicolumn{2}{|c|}{$\begin{array}{l}\text { (a) The formula formally accepted for the noted minerals do not contain } \mathrm{CO}_{3} \text {. All of the noted } \\
\text { minerals are members of the cancrinite mineral group that have the general formula } \\
\mathrm{A}_{6-9}(\mathrm{Si}, \mathrm{Al})_{12} \mathrm{O}_{24}\left[\left(\mathrm{SO}_{4}\right),\left(\mathrm{CO}_{3}\right), \mathrm{Cl}_{2},(\mathrm{OH})_{2}\right]_{2-4} \cdot \mathrm{nH}_{2} \mathrm{O} \text { where } \mathrm{A} \text { is Na, } \mathrm{Ca} \text {, and/or } \mathrm{K} \text {. The noted } \\
\text { mineral names are listed in Mandarino and } \mathrm{Back}(2004) \text { and the Internet online "Athena" } \\
\text { mineral database, but the formula listed in those sources do not include } \mathrm{CO}_{3} \text { as given above. } \\
\text { XRD patterns for the indicated minerals do not exist in the ICDD database but the comparison } \\
\text { was based on a search of only the carbonate-containing minerals in the ICDD database. }\end{array}$} \\
\hline
\end{tabular}




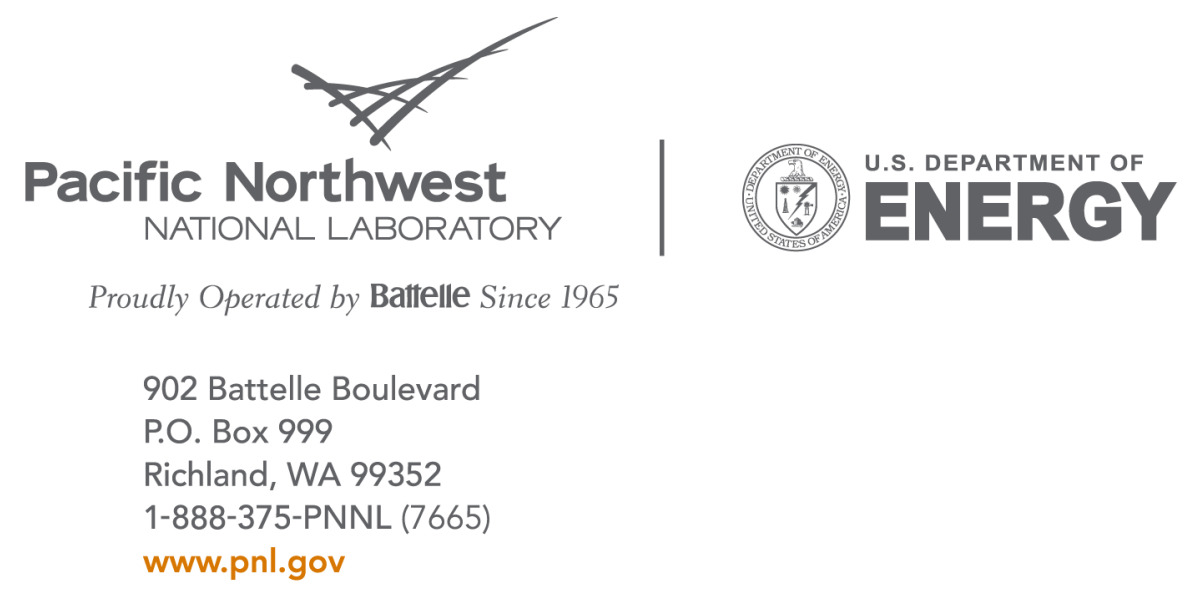

